\title{
CARBON NANOTUBE SYNTHESIS AND DETECTION: LIMITING THE ENVIRONMENTAL IMPACT OF NOVEL TECHNOLOGIES
}

\author{
By \\ Desirée L. Plata \\ B.S., Union College, 2003 \\ Submitted in partial fulfillment of the requirements for the degree of \\ Doctor of Philosophy \\ at the \\ MASSACHUSETTS INSTITUTE OF TECHNOLOGY \\ and the \\ WOODS HOLE OCEANOGRAPHIC INSTITUTION
}

June 2009

(C) 2009 Desirée L. Plata. All rights reserved. The author hereby grants to MIT and WHOI permission to reproduce and distribute publicly paper and electronic copies of this thesis document in whole or in part in any medium now known or hereafter created.

Author.

Joint Program in Oceanography/Applied Ocean Science and Engineering

Department of Marine Chemistry and Geochemistry, WHOI

Department of Civil and Environmental Engineering, MIT

May 8, 2009

Certified by

Philip M. Gschwend

Ford Professor of Civil and Environmental Engineering, MIT

Thesis Co-Supervisor

Certified by

Christopher M. Reddy

Associate Scientist of Marine Chemistry and Geochemistry, WHOI

Thesis Co-Supervisor

Accepted by

Roger E. Summons

Professor of Earth, Atmospheric, and Planetary Sciences, MIT

Chairman, Joint Committee for Chemical Oceanography

Accepted by

Daniele Veneziano

Professor of Civil and Environmental Engineering, MIT

Chairman, Departmental Committee for Graduate Students 



\title{
CARBON NANOTUBE SYNTHESIS AND DETECTION: LIMITING THE ENVIROMENTAL IMPACTS OF A NOVEL TECHNOLOGIES
}

\author{
By \\ Desirée L. Plata \\ Submitted to the Department of Civil and Environmental Engineering (MIT) \\ and the Academic Programs Office (WHOI) on May 8, 2009 in partial fulfillment \\ of the requirements for the degree of Doctor of Philosophy in the field of \\ Chemical Oceanography and Environmental Chemistry
}

\begin{abstract}
Driven by commercial promise, the carbon nanotube (CNT) industry is growing rapidly, yet little is known about the potential environmental impacts of these novel materials. In particular, there are no methods to detect CNTs in environmental matrices (e.g., sediment) and thus, there is no way to study their transport or gauge ecological exposure. Thermal methods were developed to quantify CNTs in coastal sediments down to $10 \mathrm{ug}$ per sample, which is sufficient to for CNTs in laboratory air, but not sufficient to measure contemporary levels of CNTs in the environment (which were estimated to be present at pg $\mathrm{g}^{-1}$ sediment levels using a dynamic mass balance model). In addition to the CNTs themselves, potential impacts of CNT production were assessed by monitoring emissions from a representative synthesis. An ethene-fed chemical vapor deposition process generated several compounds of environmental concern, including the greenhouse gas, methane, the hazardous pollutants, benzene and 1,3-butadiene, and toxic polycyclic aromatic hydrocarbons. By identifying critical CNT precursors (alkynes), I delivered these compounds without thermal pre-treatment and achieved rapid CNT growth. This approach reduced carbonaceous emissions by more than an order of magnitude, and lowered initial feedstock requirements and energetic demands by at least $20 \%$, without sacrificing CNT yield.
\end{abstract}

Thesis Co-Supervisor: Philip M. Gschwend Title: Ford Professor of Civil and Environmental Engineering, MIT

Thesis Co-Supervisor: Christopher M. Reddy

Title: Doherty Professor of Oceanography, WHOI 
To the people who raised me:

\author{
Candace Forsley \\ Margaret Bolduc Forsley \\ Elizabeth A. Forsley-Plata \\ and \\ Fred M. Forsley
}

Each day of my life has been blessed by these individuals.

I thank them with all the love in my heart. 


\section{ACKNOWLEDGMENTS}

Few people have the privilege of meeting their heroes. I have been blessed to work with and learn from two of mine: Prof. Philip M. Gschwend and Dr. Christopher M. Reddy. I hold each of them in high esteem for distinct but equally valued reasons, both of which are close to my heart.

Chris recognizes that the power of science can only be fully realized when the public understands it, and he has championed the communication of scientific results with the broader public. In addition to serving as a role model to his colleagues, Chris makes organized efforts to help other scientists, both young and old, improve their ability to exchange ideas across traditionally disconnected groups in our society. In doing so, he has demonstrated that state-of-the-art science and community outreach are not mutually exclusive, but rather, are synergistically beneficial.

Phil is devoted to protecting the quality of the air, water, soil, and sediment through the use of science and engineering. Through his text, he has emphasized that we can employ chemical and physical understanding to predict and prevent (!) environmental damage. Through his enthusiastic course instruction, he has recruited many others to the defense of human and ecological health. In his research, Phil maintains a scientifically rigorous approach to the study of natural processes, anthropogenic perturbations of those processes, and devising solutions to meet resultant challenges. In doing so, he has demonstrated that we can make tangible contributions to safeguard our Earth and oceans, now and into the future.

Both of these men are dedicated to their work, not for the sake of work, but for the sake of making the world a better place, and for this, I respect them immensely.

In addition to their professional qualities, both Chris and Phil are wonderful advisors and human beings. Firstly, I realize that a free-of-charge graduate education is a gift that comes at the expense of long hours of proposal writing, and I thank them for investing in me. My advisors have devoted time towards my development, not only as a scientist, but also as a professional who is poised to contribute to her field. It is clear to me that this is an uncommon gift in an advisor, and I am thankful for their dedication. I have been blessed by an overabundance of enthusiasm throughout my dissertation work, and constructive criticism was always coupled with ample motivation. This has made this project both a success and a pleasure. Lastly, I appreciate my advisors' compassion and support during personally trying times in the last six years; bless you. Phil and Chris are wonderful mentors and good friends, and I will always be grateful for them.

This project has also benefitted from several other MIT or WHOI faculty members that have served on my committee. I thank Jing Kong for her forward-thinking willingness to invite environmental chemists into her laboratory and for her continued guidance over the years. I appreciate Liz Kujawinski for providing a sound perspective on the science and direction of this project, especially with regard to the difficult manuscript submissions that we encountered. Jeff Seewald, although not an official committee member, has provided an invaluable amount of guidance on this project. He graciously allowed me to use his instruments and instructed me in the practice and principles of gas analysis. This thesis would not have been of the same caliber without Jeff, and I appreciate his direction, assistance, and good humor in the lab. Furthermore, I 
thank Jeff for serving as the chair of both my proposal defense and thesis defense and for taking an interest in this project.

The education of any graduate student does not simply derive from instructors, but also laboratory technicians and fellow researchers. I thank John "Two Pints" Andrews for his Maniac friendship and guidance in thorium flux measurements, Steve "Spike" Pike for unwavering instruction and good humor, Steve Manganini for making an impossible $\mathrm{SiO}_{2}$ measurement possible, Dave Schneider for ICP-MS instruction, Leah Houghton for comic relief and cruise assistance, Larry Ball for healthy quantities of espresso and cynicism, and Daniel Montluçon for always helping me no matter what I asked and never telling me that I was a pain. I must thank Bob Nelson for being a dear friend, fellow Yankee fan, and help in the lab from the day arrived. John MacFarlane has been an endless source of knowledge (I owe my love of wrenches and ability to speak the language of fittings to John), support, and discourse pertaining to high-quality beverages. John is a priceless resource to all of us here in Parsons. I owe the majority of my instrument knowledge to Sean Sylva, who has been a solid source of instruction, friendship, comfort, laughter, and entertainment throughout the past six years. Thanks for being there, Sean (\& Sebastian too); you are a great friend.

Anastasios John Hart has been a creative scientist with whom I am fortunate to have explored the world of nanotubes. I appreciate John welcoming me to work in his laboratory at the University of Michigan. I also thank Eoghan Reeves for his help in running and using all of the gas chromatographs in the Seewald lab. Eoghan is a remarkable analytical chemist, and I thank him for taking the time to work with me. I also appreciate helpful discussions and resources from: Gilbert Nessim, Alfonso Reina, Matteo Seita, Stevie Steiner III, and Profs. Carl Thompson, Brian Wardle, and Timothy Gutowski. Lastly, I am grateful for the opportunity to have met and experimented with Eric Meshot at the University of Michigan. Eric is an extraordinary spirit who is both fun and inquisitive, and he inspires me to pursue science with fervor. I appreciate his tolerance of my brazen approaches to getting things done and all of the nights of sleep he missed working to complete this research. Mostly, I am thankful for his friendship.

The Joint Program is a unique and amazing graduate program that owes its exceptionality to a handful of individuals working to administer the program at MIT and WHOI. Julia Westwater, Marsha Gomes, Valerie Caron, and Christine Charette are thanked for their dedication to making WHOI a home away from home, where we are always welcomed and supported. (Julia, bless you for all the hugs and smiles!). I also thank John Farrington, Judy McDowell, Jim Yoder, Jim Price, and Meg Tivey for building and maintaining a strong academic program that draws on the resources of two institutions, maximizing benefits to students and minimizing any adverse impacts on students' daily lives. The conversations, both personal and professional, that I have had with these individuals have strengthened me as a scientist and as a human being. Ronni Schwartz and Paola Rizzoli's efforts to provide a strong base for the Joint Program at MIT are greatly appreciated. Whether I needed a car, research funding, or simply a boisterous salutation from a women who followed her scientific dreams in a time when it wasn't all that common, they were there for me. I thank all of these individuals for enriching my life and facilitating my exploration.

As a member of the Parsons Laboratory at MIT, I have been blessed by several other people; Vicki Murphy, Jim Long, and Sheila Frankel. They go aboveboard to make day- 
to-day operations efficient and enjoyable. Their efforts to make Parsons a functional, happy, and environmentally conscientious (!) workspace are unmatched, and perhaps miraculously, very effective. Vicki, Jim, and Sheila were always ready to help me at the drop of a pen, with financial assistance, photographic/graphic advice (which is increasingly important in science), or a compliment when my feet may have been dragging. I am grateful to know and work with them.

I thank my teachers, past and present, for helping make research something that I love. From elementary school: Peter Walgreen, for taking the time to invest in me; Paul Ranucci, for knowing just what a kid wanted to talk about; and Pat Niles, for helping me advance my studies and, perhaps most importantly, teaching me to love the Earth. From Gould Academy: Annette Brickley, for encouraging, loving, and challenging me; Erik Janicki, for teaching me to be proud of my nerdiness and merge it with the outdoors; Robin Lee, for teaching me the proper way to argue; and to Lorenzo, for giving me a nod on the way out and a nice couch to come back to. From Union College: Tom Werner, for helping us use chemistry to study the environment; Les Hull, for pushing me whenever he had the chance; Dave Hayes, for bringing a free spirit to physical chemistry and teaching me that writing is not as subjective as I thought; R. Chris Schnabel, for being all over the place, and in doing so, exposing us to so much fantastic science; and Mary Carroll, for advising me differently than others and teaching me nearly everything I know about being a professional in chemistry. From MIT/WHOI: Dan Repeta, for challenging us to choose important problems and defend our ideas; Bernard Peucker-Ehrenbrink, for his enthusiasm, rigor, and excitement for the creation of the elements; Dave Glover, for never stopping his instruction, even after class was over; Roger Francois, for teaching me about the oceans; Scott Doney, for reminding me that people on an airplane will expect me to know everything about the ocean as it relates to them; and Phil Gschwend, for crafting and teaching the most personally meaningful class I've ever taken. I'm privileged to have learned from these exceptional teachers.

I would not be who I am without the friends that I have made in my life. I thank Kara Smith Lopez and Sarah Crocker for being my oldest and dearest friends. I am grateful to count them among my sisters, and I hope they know how much they have shaped me throughout my life. Justin Gray, Bill McCloy, and Mark Balcar are appreciated for adventures in high school and beyond. Allison Stevens, Joanna Doxey, Carolyn Simon, and Matt Blackwell have always been there to support me and have been so understanding of my ridiculous schedule; I thank them for the laughter and conversations over the years. Bob Herbst, thank you for making chemistry and college much better than they would have been without you; you're a special person, and I appreciate you. Kristin Pangallo (my academic Irish twin), Eoghan Reeves (who's actually Irish), James Saenz (who thinks he's Scottish), Francesca DeLeonardis, and Laura Hmelo are thanked for making Fye more fun, for spectacular times in the trailer, and for trips to DQ. Casey Saenger, you are simultaneously an amazing and normal human being; I thank you for the conversations, encouragement, and groundedness you have brought to my life during grad school. My friends in Parsons- Loretta Fernandez, Matt Orosz, Lejo Flores, Xanat Flores-Cervantes, and Amy Meuller- have inspired me to be a scientist who uses her time to the do the most good for others, and I thank them for that. I am in awe of them as individuals. Xanat and Amy are especially thanked for being welcoming and kind to me when I first arrived as a shy stranger from WHOI; you are magnificent human beings. 
Lejo, I thank you for being so relatable, fun, and willing to adventure out of the cubes with me. Dreux Chappell, Paul Craddock, and Christian Miller; I don't know what I would have done without my core classmates, and I appreciate your friendship immensely. Ari Shapiro, you are an angel, and I hope you will forever be as wonderful and happy as you have been since the moment I met you. Nick Drenzek, you were the one true friend I made when I first arrived as a Summer Fellow at WHOI; thank you for being a caring, upstanding individual. I owe the ultimate trajectory of my graduate career to the guidance of Helen White and Natalie Goodkin Emami. These women helped give me the courage to pursue off-the-beaten-path research in an off-the-beaten-path way, and I thank them immensely for their counsel and support. My graduate experience would not have been as spectacular or fulfilling as it was if it weren't for their encouragement.

There are a group of people in my life who all but define who I am today, and I know will be by my side into the future. My cousins and siblings are my best friends, and they have been since my time began. They are individually some of the most extraordinary people I know, who have big hearts and an even bigger sense of adventure. They never give up on their pursuits or dreams, regardless of what roadblocks end up in their way. What's more is that they will never give up on one another. My cousins and siblings have contributed and sacrificed so much for my betterment. First, I am infinitely more "normal" than I would have been without their social guidance (and tolerance of my determined efforts to be anything but normal). Second, in times when I was most in need, they have always cheered my spirits simply by being there for me, adventuring with me, and loving me. Third, and most notably, each of them has had to share their homes and parents with me at some point in time (no small sacrifice for a child). Not one of them has ever complained or whined about this, even though they would have been justified to do so. Jason, Ryan, Ben, Eli, and Abigail, I love you more than you'll ever know and I thank you for being so good to me. You're great.

I have been blessed to have an entourage of parents. Each has taught me distinct and important life lessons, and each has shown me unconditional love. My mother, Elizabeth, has taught me to find the extraordinary in the routine, to appreciate every individual, and that there is always a means to an end, regardless of the path you take to get there. My mother's larger-than-life perspective on the world served me well as a child and an adult, as I was brainwashed into believing that I could do anything. Outside of that, and teaching me how to read, she gave me no other instruction or preconceptions- the perfect formula to create a young scientist. My mother's sister, Candace, was also my mother from the day I was born. My auntie taught me how to be thankful, graceful, and kind, how to show love, how to know when someone needs you, how to help them feel better, and how to laugh with someone you hold dear. My aunt was there for me, even when we were apart, and she will be always. She was my biggest fan, and I am hers. My aunt Margaret was my mother too, and she provided a safe and loving home for me when I most needed it. She expects nothing in return, and always goes aboveboard for her children. I am fortunate to be counted among them. I thank her for being so constant, so dependable, and so loving. Lastly, and mostly, is my Dad (uncle Freddy). From the time he was about 26 , he came to get me every weekend to do business, deliver beer, practice math in the car, go skiing, go to work, and visit our family. He is responsible for keeping me interested in school, in sports, and pushing me to pursue my dreams. My love of the ocean is derived from our walks on the beach and rides in leaky 
boats on Casco Bay, and I would not be an Oceanographer if it weren't for him. He is one of the most amazing human beings I know. Of all the teachers that I have had in my life, he has taught me the greatest lesson: Think positive and make the best of everything. I would not have made it through life with a smile on my face without him. I love him more than words can say.

Ten years ago, I met a man that was really fantastic at organic chemistry, and I thought that was delightful. Then, I realized that he had a kind heart and a genuine spirit, and I thought that he was even more amazing. Ricky is thoughtful, compassionate, and caring, brilliant and unassuming. He shares my love for the Earth and for trying to help others feel a little bit better each day. He has been there for me (and my family) and stuck with me through some very trying times. I love his whole heart and soul with my whole heart and soul. Thank you for being there, angel.

This work was supported by the taxpayers of the United States of America and the benefactors of MIT and WHOI. Specifically, I thank the National Science Foundation (NSF) Graduate Research Fellowship Program, NSF OCE-0221181, the Seaver Institute, the Martin Family Society of Fellows for Sustainability, Arunas and Pam Chesonis through MIT's Earth System Initiative, and the WHOI Ocean Ventures Fund. Without these monies, this work, and my education, would not have been possible. I am forever grateful. 


\section{TABLE OF CONTENTS}

Chapter 1: General Introduction:

A New Approach to the Design of Novel Anthropogenic

Materials...................................................... 21

Introduction................................................. 22

Background............................................... 24

Characteristics of carbon nanotubes....................... 24

CNT synthesis..................................... 26

CNTs in the environment and similarity to BC............. 28

Estimated environmental concentrations of CNTs................. 32

Thesis overview.......................................... 34

References................................................. 36

Supporting information..................................... 40

Chapter 2: Industrially synthesized single-walled carbon nanotubes:

Compositional data for users, environmental risk assessments, and source

Apportionment...................................................... 45

Abstract..................................................... 46

Introduction................................................. 46

Experimental methods......................................... 50

Samples........................................... 50

Metal, metalloid, and rare earth element analysis............ 50

Determining bulk carbon and nitrogen content of SWCNTs... 51

Determining stable carbon isotopic content of SWCNTs..... 51

Dichloromethane and toluene extractions.................... 52

Extractable polycyclic aromatic hydrocarbons........ 52

Stable carbon isotope content of extractable carbonaceous material........................... 53

Results and Discussion........................................ 54

Metal and metalloid content................................ 54

Expected and unexpected metals........................... 54

Unique metal ratios for SWCNT source apportionment...... 57

Total carbon and nitrogen............................... 59

Stable carbon isotope composition of SWCNTs.............. 65

Total solvent-extractable material (TSEM).................. 67

TSEM isotopic composition and hydrocarbon origin.......... 68

TSEM mass and metal reactivity......................... 68

Solvent-extractable polycyclic aromatic hydrocarbons........ 72

Implications and conclusions................................. 74

Acknowledgments........................................... 75

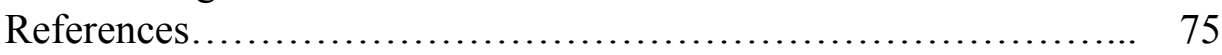

Supporting information........................................ 79 
Chapter 3: Analysis of single-walled carbon nanotubes in environmental matrices by thermogravimetry- mass spectrometry....... 81

Abstract......................................................... 82

Introduction............................................... 83

Experimental methods....................................... 84

Samples............................................ 84

Thermogravimetric analysis- mass spectrometry............ $\quad 85$

Data processing.............................. 86

Correction for TPOs........................ 86

Correction for HATDs.................... 86

Blanks and precision of mass and temperature......................... 87

Results and Discussion...................................... 87

TPO of SWCNTs and natural organic material............ 87

TPO of complex environmental matrices................... 93

TPO-MS: Unique ion ratios from SWCNTs............... 93

HATD of SWCNTs, natural organic materials, and complex environmental matrices....................... 96

HATD-MS: Unique ion ratios from SWCNTs............. 98

Current applications of TGA-MS to detect SWCNTs in the environment................................ 101

Acknowledgements......................................... 103

References................................................. 103

Supporting Information................................. 106

Potential oxidizing power of SWCNT metal catalysts....... 106

Mass spectra of evolved gases from the thermal treatment of standard materials........................ 107

MWCNT and C60 thermal stability during TPO and HATD.

Attempts to reduce interferences in SWCNT oxidation temperature using different thermal programs.

Stable carbon isotopic content as a tool to quantify SWCNTs in complex mixtures

Mass fragments generated during the hydrogenassisted thermal decomposition of SWCNTs and other test materials.

Chapter 4: Early evaluation of potential impacts of carbon nanotube synthesis by chemical vapor deposition........................... 127

Abstract.................................................. 128

Introduction............................................... 128

Results and Discussion...................................... 135

VOCs formed during VA-MWCNT synthesis:

Effects of pre-heater temperature. 
VOCs formed during VA-MWCNT synthesis:

Effects of hydrogen gas content

138

Safety concerns and environmental implications of extrapolating VOC emissions to industrial scale.........

PAH formation during VA-MWCNT synthesis: Effects of temperature and hydrogen gas content.........

PAH safety concerns and environmental implications of extrapolating to industrial scale.

Solid carbon formation during VA-MWCNT synthesis: Effects of temperature and hydrogen gas content.

Additional carbonaceous materials in the effluent contribute to smog formation....

Extension to alternative CNT production methods 150

Conclusions and implications for future CNT production Management. .

Experimental methods.

152

CNT synthesis....................................... 152

VOC collection and analysis........................... 153

PAH collection and analysis............................. 154

Pre-heater tube deposits and adsorbed PAHs............... 154

Acknowledgments.......................................... 155

References................................................ 155

Supporting information................................... 162

PAHs are retained on cool, room temperature walls of quartz reactor tube

162

Scaling VA-MWCNT production to potential markets.

Scaling VA-MWCNT production to the size of the current liquid crystal display market...

Scaling VA-MWCNT production to the size of the current household computer central processing unit market

Unique PAH isomers present in the CVD effluent........... 166

Effects of temperature on substituted benzene formation.

Identification of compounds formed by thermal treatment of reactant gases........................... 168

Fate of 1,3-butadiene and benzene in the atmosphere........ 169

References......................................... 169

Chapter 5: Improved resource use in carbon nanotube synthesis: Rapid growth without thermal treatment of feedstock gases................. 171

Abstract....................................................... 172

Introduction................................................. 173

Experimental methods................................... 175 
Chemical supplies.................................... 175

CNT synthesis....................................... 175

VOC collection and analysis.............................. 176

PAH collection and analysis........................... 177

Results and Discussion..................................... 178

Accelerated CNT growth with thermal pre-treatment of feedstock gases.................................... 178

Accelerated CNT growth without heating feedstock gases................................................ 180

Reduced by-product formation without sacrificing CNT growth...................................... 183

Minimizing the use of ethene and hydrogen: Optimizing feedstock requirements.................... 187

Acknowledgements....................................... 190

References............................................. 191

Supporting Information.................................... 194

Linear and log-linear relationships between CNT precursor molecule abundance and CNT growth rate.............. 194

References................................................ 201

Chapter 6: Reflections on and forecasts of this work: Contributions, criticisms, precedent, and opportunities.......................... 203

Contributions............................................... 204

Potential impacts from the manufacture of CNTs........... 204

Developing a method to detect CNTs in the natural world .......................................... 208

Multi-walled CNTs vs. single-walled CNTs................ 209

Criticism.......................................................... 212

Precedent................................................. 216

Opportunities................................................ 217

References.............................................. 222 


\section{LIST OF FIGURES}

Chapter 1: General Introduction:

A New Approach to the Design of Novel Anthropogenic Materials.......

Figure 1. CNT structures...................................... 25

Figure 2. CNTs and BC soot................................. 31

Figure 3. Estimated SWCNT concentrations in Galveston

Bay and Houston, TX................................. 33

Chapter 2: Industrially synthesized single-walled carbon nanotubes:

Compositional data for users, environmental risk assessments, and source apportionment

Figure 1. Metal ratios in SWCNTs and other aerosol sources...... 58

Figure 2. Total accounted SWCNT mass as a function of assumed metal content........................................... 64

Figure 3. Stable carbon isotopic signatures of SWCNTs,

SWCNT-associated solvent extractable material, and

selected carbon sources.................................. 66

Figure 4. Polycyclic aromatic hydrocarbon internal standard

Recoveries............................................... 71

Figure S1. CNTs in various organic solvents................... 79

Chapter 3: Analysis of single-walled carbon nanotubes in

environmental matrices by thermogravimetry- mass spectrometry.........

Figure 1. Oxidation thermograms of SWCNT powders............ 89

Figure 2. Maximum oxidation rate temperature as a function of metal content in SWCNTs................................ 91

Figure 3. Oxidation thermograms of representative environmental materials................................ 92

Figure 4. Diagnostic ion ratios observed in thermal oxidations of SWCNT-amended coastal sediments............. 95

Figure 5. HATD thermograms of SWCNT powders............... 97

Figure 6. HATD thermograms of representative environmental materials.......................................... 99

Figure 7. Diagnostic ion ratios observed in HATD of SWCNT-amended coastal sediments........................ 100

Figure 8. Sample-derived 28 signal increases with SWCNT content of SWCNT-amended sediments...................... 92

Figure S1. Mass spectra of evolved gases from the TPOs.......... 108-109

Figure S2. Thermograms of C60 and MWCNTs................. 110

Figure S4. Thermal program Test \#1 with sediments.............. 111

Figure S5. Thermal program Test \#1 with SWCNTs and NIST 1650

Figure S6. Thermal program Test \#2 with SWCNTs and

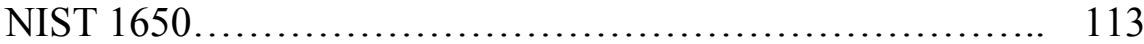

Figure S7. Thermal program Test \#3 with sediments.............. 114

Figure S8. Thermal program Test \#4 with sediments.............. 115 
Figure S9. Stable carbon isotopic content in sediments and

SWCNT-amended sediments............................ 116

Figure S10. Calculated CNT content following thermal

oxidation

Figure S11. Mass spectra of evolved gases from the HATDs.......

Figure S12. SWCNT degradation does not release $\mathrm{N}_{2} \ldots \ldots \ldots \ldots \ldots, 123$

Figure S13. Ethene calibration curve.......................... 125

Chapter 4: Early evaluation of potential impacts of carbon nanotube

synthesis by chemical vapor deposition.

Figure 1. Co-operative approach to material design.............. 131

Figure 2. Reactor design and sampling scheme................... 134

Figure 3. VOC abundance as related to pre-heater current.......... 136

Figure 4. Effect of pre-heater temperature on growth

parameters........................................... 137

Figure 5. VOC abundance as a function of hydrogen content...... 139

Figure 6. PAH content as a function of temperature............... 143

Figure 7. PAH content as a function of hydrogen................. 144

Figure 8. Structures of observed VOCs and PAHs................ 149

Figure 9. Two-dimensional gas chromatograph of

hydrophobic materials in the effluent...................... 150

Figure S1. PAHs content before and after the reactor tube........ 162

Figure S2. Acephenanthrylene, a rare isomer of pyrene and

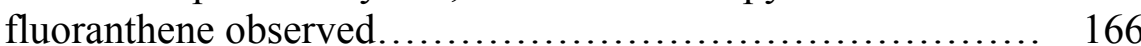

Figure S3. Two-dimensional gas chromatograph of

hydrophobic materials as a function of temperature........... 167

Chapter 5: Improved resource use in carbon nanotube synthesis: Rapid

growth without thermal treatment of feedstock gases

Figure 1 . Thermally generated compounds correlated with

increase CNT growth...................................... 179

Figure 2. Effects of test gas on CNT growth................... 182

Figure 3. Reduced VOC formation without thermal

treatment of feedstock gas.

Figure 4. Reduced PAH formation without thermal

treatment of feedstock gas............................... 186

Figure 5. Effects of ethene and hydrogen on CNT growth......... 188

Figure S1. Kinetic treatment of propyne's effect on

VA-MWCNT growth rate

Figure S2. Diagram of experimental apparatus.................. 195

Figure S3. Proposed CNT formation mechanism............... 200-202

Chapter 6: Reflections on and forecasts of this work: Contributions, criticisms, precedent, and opportunities.

Figure 1. High-resolution TEM of CNTs grown in the presence and absence of PAHs.................................. 207

Figure 2. A flame tower at a CNT production facility............ 213 


\section{LIST OF TABLES}

Chapter 1: General Introduction:

A New Approach to the Design of Novel Anthropogenic Materials

Tables 1-3: Information to calculate CNT concentration in

Houston box model.

$41-43$

Chapter 2: Industrially synthesized single-walled carbon nanotubes:

Compositional data for users, environmental risk assessments, and source apportionment

Table 1. US SWCNT manufacturers............................... 49

Table 2. Detection limit of PAHs extracted from SWCNTs........ 53

Table 3. Bulk metal and metalloid content of SWCNTs........... 56

Table 4. Bulk $\mathrm{C}$ and $\mathrm{N}$ content of SWCNTs..................... 61

Table 5. Mass of solvent-extractable material form SWCNTs... $\quad 70$

Table 6. PAHs extracted from SWCNTs.......................... 73

Chapter 3: Analysis of single-walled carbon nanotubes in environmental matrices by thermogravimetry- mass spectrometry

Table 1. SWCNTs used in this study ........................... 85

Table S1. Reduction potentials of metals used in CNT synthesis... 106

Chapter 4: Early evaluation of potential impacts of carbon nanotube synthesis by chemical vapor deposition

Table 1. Projected emissions due to CVD CNT synthesis.

Table 2, 3. Carbonaceous deposit formed in quartz pre-heater as a function of temperature, hydrogen content................... 147

Table S1. Total liquid crystal display area....................... 164

Table S2. List of compounds and analytical methods used to identify the compound.................................. 168

Chapter 5: Improved resource use in carbon nanotube synthesis: Rapid growth without thermal treatment of feedstock gases

Table S1, S2: VOC and PAH content as related to test-gas .........

Chapter 6: Reflections on and forecasts of this work: Contributions, criticisms, precedent, and opportunities.

Table 1. CNT market size distribution. 


\section{LIST OF ACRONYMS}

CNT.

CPU.

CVD

FID.

FPD

GC

$\mathrm{GC} \times \mathrm{GC}$

HATD

LCD

MS

MWCNT

PAH.

SWCNT

TCD.

TGA

TIM

TOF

TPO

VA.

VOC.
Carbon nanotube

Central processing unit

Catalytic chemical vapor deposition

Flame ionization detector (or detection)

Flat panel display

Gas chromatography

Comprehensive two-dimensional gas chromatography

Hydrogen-assisted thermal decomposition

Liquid crystal display

Mass spectrometry

Multi-walled carbon nanotube

Polycyclic aromatic hydrocarbon

Single-walled carbon nanotube

Thermal conductivity detector

Thermo gravimetric analysis (or analyzer)

Thermal interface material

Time-of-flight

Temperature-programmed oxidation

Vertically-aligned (e.g., VA-MWCNT)

Volatile organic compound 


\section{CHAPTER 1:}

GENERAL INTRODUCTION:

A NEW APPROACH TO THE DESIGN OF

NOVEL ANTHROPOGENIC MATERIALS

\section{By}

Desirée L. Plata 


\section{INTRODUCTION}

Carbon nanotubes (CNTs) are a promising new material whose production is increasing rapidly. Historically, no industrially important chemical has been synthesized, used, and disposed of without some release to the environment (e.g., dichloro diphenyl trichloroethane (DDT), polycholorinated biphenyls (PCBs), polybrominated diphenyl ethers (PDBEs), or methyl-tert-butyl ether (MTBE)). Similarly, by-products of largescale syntheses are often unintentionally discharged to the air, land, and nearby waterways (e.g., perfluorooctanoic acid (PFOA) from Teflon ${ }^{\circledR}$ manufacture and dioxins from polyvinylchloride (PVC) production). Thus, as the CNT industry expands, one can expect to see an increase in environmental levels of (1) CNTs and (2) co-products of CNT manufacture.

Currently, there are no established procedures to quantify CNTs in environmental matrices (e.g., air, water, or sediment). As a result, there is no way to gauge the release of these materials or study their environmental fate. Furthermore, early investigations suggest that CNTs may be toxic, and the development of a means to determine ecological and human exposure to CNTs is critical. Thermal techniques have been used to quantify recalcitrant carbon forms (e.g., black carbon $(\mathrm{BC})$ ) in the environment, and CNTs share many structural and physical similarities with BC (e.g., high thermal stability relative to most organic matter). I hypothesized that CNTs could be detected and distinguished from other organic carbon using a thermal analytical method. Such an approach would provide a low-cost, high-throughput analysis for the quantification of this engineered material in the natural world. 
The environmental impact of CNT production is not limited to the release of CNTs, but includes emissions from CNT synthetic practices. The industrially favored production method, catalytic chemical vapor deposition (CVD), is plagued by low efficiency, suggesting that large amounts of carbonaceous by-product or un-reacted feedstock may be released to the atmosphere. Given that typical CVD methods require heating carbon source gases to high temperature (e.g., $700-1000^{\circ} \mathrm{C}$ ), I hypothesized that the CVD effluent would contain compounds of environmental concern, including CNT fragments, $\mathrm{BC}$, polycyclic aromatic hydrocarbons (PAHs), and volatile organic compounds (VOCs). Furthermore, by developing a chemical understanding of the dominant CNT formation mechanisms, I expected that I could design a synthetic approach that minimized the content of dangerous material in the effluent.

As the CNT industry is poised for (or arguably in the midst of) rapid growth, there is a critical need to understand the potential impacts of CNT release and consequences of industrial-scale synthesis prior to significant monetary investment or environmental damage. In this thesis, I strived (1) to develop a method to monitor the fate of and biological exposure to CNTs in complex environmental matrices (e.g., air, water, soil, and sediment), and (2) to assess emissions from a common CNT production method and reduce the release of compounds of environmental concern (e.g., green house gases, toxins, and materials that form secondary pollutants, such as smog and aerosols). When possible, I rapidly communicated these results to industry and government, and I continue to encourage them to adopt practices to lessen the environmental impacts of this burgeoning industry. In this way, I sought to prevent unacceptable ecological and human 
health consequences due to material manufacture, and thereby alter the, so far, unfortunate trajectory of industrial chemicals in the Earth system.

\section{BACKGROUND}

Characteristics of carbon nanotubes. CNTs are hollow cylinders of $s p^{2}$ hybridized carbon atoms arranged in repeating hexagonal rings (Figure 1). They are aromatic, resembling rolled up graphene sheets with hemi-fullerene caps. CNTs may consist of a single cylinder (single-walled carbon nanotubes (SWCNTs)) or multiple, concentric cylinders (multi-walled carbon nanotubes (MWCNTs)), where the former have smaller diameters $(0.7-2 \mathrm{~nm})$ than the latter (diameters ranging from 8 to $40 \mathrm{~nm}$ ). Regardless of the number of tube walls, high van der Waals forces between individual tubes cause them to agglomerate into CNT "ropes" or randomly ordered free-floating particles ${ }^{l}$. These CNT "powders" are distinct from ordered CNT structures (e.g., vertically-aligned CNTs or CNTs grown on scaffold networks), which can be produced for specific industrial applications on large ( $\mathrm{mm}$ to $\mathrm{cm}$ scale), solid substrates. The macroscale configurations of CNTs are as diverse as their potential and demonstrated applications, and these CNT structures will influence the ultimate environmental fate of the nanomaterials. 


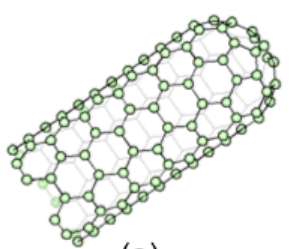

(a)

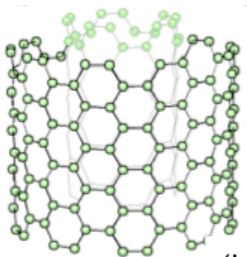

(b)

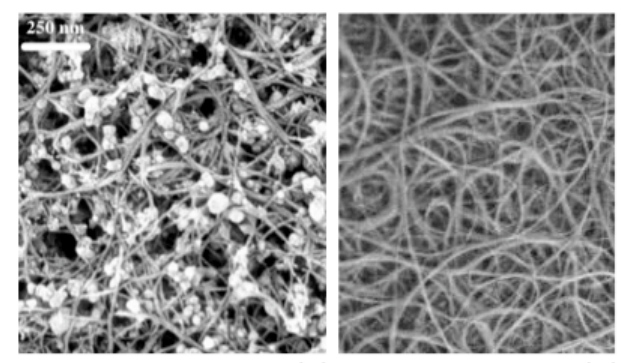

(c)

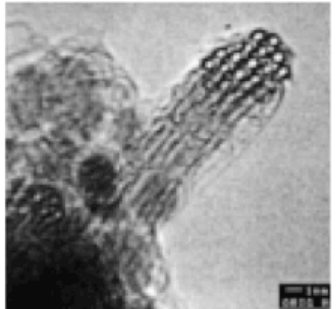

(e)

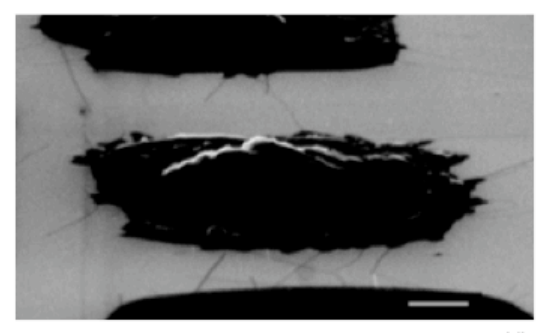

(f)

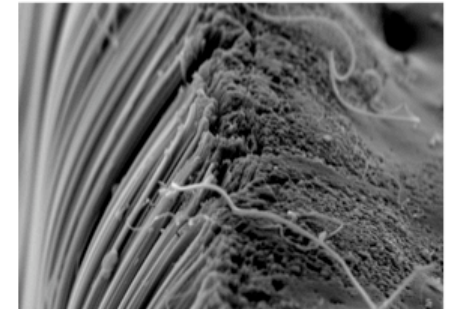

(g)

Figure 1. Ball-and-stick model representations of (a) single-walled carbon nanotubes (SWCNTs) and (b) multi-walled carbon nanotubes (MWCNTs) (www.photon.t.utkyo.acljp/ maruyama/fticr/ft-icr.html). Scanning electron micrographs of (c) unpurified SWCNTs (where the white spheres are metal catalyst clusters) and (d) purified SWCNTs (www.fibrils.com) powders. High-resolution transmission electron micrograph of (e) a SWCNT rope (Nicolaev et al. ${ }^{12}$ ) in a SWCNT powder. Ordered CNT structures can be grown (f) between silicon island scaffolds (SWCNTs, Kong et al. ${ }^{11}$ ) or (g) in vertical alignment (MWCNTs, www.nanobliss.com). 
The industrial promise of CNTs arises from their exceptional mechanical strength, high thermal stabilities, heat transfer characteristics, sorptivity, unique optical properties, and ability to support large current densities ${ }^{2}$. Current CNT-based consumer products rely on epoxy-encapsulated MWCNTs and include sporting equipment (baseball bats, bicycles, tennis rackets, and sailboat masts $)^{3}$, as well as conductive plastics, which have been produced and sold for over twenty years ${ }^{4}$. In the future, CNTs are expected to offer breakthroughs in the electronics industry, from improved energy storage to faster super computers, meeting the demands of today's challenging scientific questions via enhanced computational power and bringing us toward the common goal of carbon neutral energy production through advanced battery technology. In addition, CNTs may enable noninvasive monitoring of blood components (e.g., glucose for diabetics), as well as cellspecific drug delivery (e.g., for cancer patients). These significant, potentially lifesaving, societal benefits may prove indispensible, and it is critically important that CNTs develop in a sustainable fashion that will not threaten their commercial longevity. Given the diverse and powerful proposed uses of CNTs, the market is expected to expand from $\$ 215$ million in 2009 to $\$ 1,070$ million in 2014, implying a doubling rate of once every two years ${ }^{5}$ (and assuming no change in the dollar-to-mass CNT ratio over the next five years). Historically, increased production of industrial chemicals augments their release to the air, water, and soil ${ }^{6}$, and it is reasonable to expect that environmental levels of CNTs and their by-products will mimic the growth in CNT production volume.

CNT synthesis. Since the recognition ${ }^{\mathrm{a}}$ of CNT structures in $1991^{10}$, the principle challenge limiting the commercialization of CNT-based products was the ability to synthesize the materials in significant quantities. This challenge was met in 1998, with the development of CVD techniques ${ }^{11,12}$. CVD syntheses involve the introduction of a carbon-vapor feedstock $\left(\mathrm{CH}_{4}, \mathrm{CO}, \mathrm{C}_{2} \mathrm{H}_{4}, \mathrm{C}_{2} \mathrm{H}_{2}\right.$, etc.) to an $\mathrm{O}_{2}$-free heated zone (500$1000^{\circ} \mathrm{C}$ ) where catalytic metal particles (e.g., Fe, Co, Mo, or Ni) are free-floating or fixed

\footnotetext{
${ }^{\text {a }}$ MWCNTs were first reported in the late 1970s but were referred to as "fibers" made of crystalline carbon layers "wrapped together" 7-9.
} 
to a stationary substrate. The resultant product is a mixture of CNTs, metal catalyst, and carbonaceous impurities that are often referred to as "amorphous carbon"12,13. Attempts to remove the metallic and carbon-based co-products often involve oxidative processes that damage the CNT lattice, and purification techniques represent a compromise between impurity removal and product quality. Thus, "pure" carbon nanotubes that are free of co-products are not yet a commercial reality ${ }^{14}$.

Large volume CVD reactors are capable of producing kilograms of CNT material per day, and current global production is roughly 300 tons $\left(3 \times 10^{8} \mathrm{~g}\right)$ per year ${ }^{15}$. Although the rate of environmental release of CNTs is unknown, we suspect that the reduced, highly aromatic carbon will be relatively recalcitrant after it is introduced to environmental settings. Thus, while the annual mass of CNT released to the environment may be small relative to other carbon fluxes (e.g., BC fluxes are on the order of $\mathrm{Tg}$ ( $\mathrm{Tg}=$ $\left.10^{12} \mathrm{~g}\right)$ ), the accumulation of CNTs over time could become important.

CNT-carbon only represents a fraction of the carbonaceous material that goes into CNT production. Although there have been few comprehensive mass-balances of a carbon feedstock through a CVD process, it is believed that less than $3 \%$ of the introduced carbon yields CNT product ${ }^{16}$. Unreacted feedstock and by-products are often vented to the atmosphere ${ }^{b}$. If we assume a maximum efficiency of $3 \%$, then untreated effluents could result in an annual release of $10 \times 10^{9} \mathrm{~g}$ of carbonaceous material (growing to $0.3 \times 10^{12} \mathrm{~g} \mathrm{year}^{-1}$ by 2019). These effluents were completely uncharacterized prior to this study ${ }^{17}$, but the high reaction temperatures suggested that the composition would be distinct from the feedstock gas. In particular, high-temperature, gas-phase carbonaceous reactions may promote the formation of VOCs, PAHs and BC soot, all of which raise environmental and public health concerns. VOCs are primary pollutants that can be acutely toxic (e.g., benzene or 1,3-butadiene) or act as greenhouse gases (e.g., methane). They also exacerbate the formation of secondary pollutants, such

\footnotetext{
${ }^{\mathrm{b}}$ Unidym (formerly Carbon Nanotech, Inc.), SouthWest Nanotechnologies, and Nanocyl recycle their effluents for use in more than one CNT batch, but ultimately (after recycle) release non-CNT material to the atmosphere. Nanocyl flames (combusts) any un-recycled effluent before release to the atmosphere.
} 
as smog, which impacts respiratory health. PAHs are toxic, persistent, bioaccumulating compounds that affect both public and ecological health, while $\mathrm{BC}$ soot influences the global heat budget, either by acting as light-absorbing particles and reducing the reflectance of ice caps. BC soot can also influence the fate of hydrophobic organic contaminants and act as a pulmonary toxicant itself. Without accurate identification and quantification of the effluent constituents, we can neither describe nor mitigate the impact of CNT production on health of the oceans, atmospheres, ecosystems, or society.

CNTs in the environment and similarities to $B C$. A recent report by the National Research Council ${ }^{18}$ highlighted that programs to assess the risks associated with nanomaterials and their production are severely deficient. In spite of substantial investments in CNT-based applications research ( $>5,000$ journal articles), fewer than 100 studies have been devoted to assessing the potential environmental impacts of CNTs and only a handful discuss the effects related to CNT manufacture ${ }^{19}$. While sparse, preliminary toxicological studies suggest that CNTs are cytotoxic to alveolar macrophages $^{20,21}$, may result in pre-cancerous, pulmonary granulomas ${ }^{22}$, and adversely affect the life cycle development of marine organisms that play a critical role in nutrient cycling in the upper ocean ${ }^{23}$. These effects have been attributed to the metals associated with $\mathrm{CNTs}^{24}$, carbonaceous impurities ${ }^{23}$, the shape of the CNTs (whose long aspect (length: diameter) ratio is similar to asbestos ${ }^{22}$ ), as well as the functional groups on the CNT surface ${ }^{25}$.

In addition to their own toxicity, CNTs can act as vectors of toxic molecules across cellular membranes that are otherwise impenetrable ${ }^{26}$. CNT-uptake into the cell is via phagocytosis ${ }^{26}$, rather than via diffusion across an activity gradient. Indeed, as particles, rather than molecules, CNTs will not partition into biological media (e.g., cells and lipids of macrofauna) in a classical sense, but will instead bioaccumulate via physical ingestion of the particles (e.g., filter feeding ${ }^{23}$ or phagocytosis). Thus, equilibrium partitioning models will not describe CNT exposure accurately, and bioaccumulation models will have to incorporate considerations of particle transport as well as feeding mechanisms. 
If CNTs have a natural source and have been present in the environment over evolutionary timescales, it is possible that organisms have some capability to either degrade or excrete potentially harmful CNTs. Murr et al. ${ }^{27,28}$ argue that CNTs have a natural source, reporting CNTs in contemporary and ancient air samples. Although these were observed by electron microscopy using statistically insignificant sample sizes (individual water droplets), it is possible that CNTs have been produced throughout history. The contribution of natural CNTs to a recalcitrant carbon pool cannot be assessed until an analytical method is developed for samples that will provide a historical record, such as coastal marine sediments. If natural CNTs have been present in the environment, it is possible that they have been quantified with another refractory form of carbon, $\mathrm{BC}$.

BC soot and CNTs share many chemical and physical characteristics (Figure 2). Both are made up of aromatic, graphitic structures and exist as black, low-density powders that are difficult to distinguish by visual observation alone. However, the two materials are clearly distinct when investigated by electron microscopy. $\mathrm{BC}$ soot has a highly condensed configuration with multiple, stacked graphene sheets that are spaced by $0.34 \mathrm{~nm}^{29}$. These stacks are layered in an "onion-like" fashion that forms $30-50 \mathrm{~nm}$ spheres, which agglomerate to form "grape-like" arrangements. CNTs, in contrast, are long (um to mms), hollow cylinders of narrow diameter $(0.7-40 \mathrm{~nm}$ ). (Concentric CNT cylinders that form MWCNTs also have $0.34 \mathrm{~nm}$ spacing between individual CNT walls, similar to the spacing in graphene layers in $\mathrm{BC}$ soot). These tubes can either be vertically aligned or exhibit curvature, resembling tangled, "spaghetti-like" structures. These structural differences may give rise to distinct physical properties that could be used to distinguish the two materials in complex mixtures. For example, the relative proportion of carbon on the surface of a CNT is greater than that of BC soot, which has a substantial mass of carbon stored in interior layers. Thus, chemical reactants, such as diatomic oxygen $\left(\mathrm{O}_{2}\right)$, have reduced access to the majority of carbon in BC soot. CNTs, and especially SWCNTs, might be more susceptible to complete transformation by oxidation in engineered systems (e.g., temperature-programmed oxidations or other thermal 
analyses) than $\mathrm{BC}$ soot, resulting in a lower thermal stability of CNTs relative to the otherwise similar BC.

In spite of nanoscopic structural differences, $\mathrm{BC}$ soot and CNTs are expected to have analogous surface chemistry, optical properties, hydrophobicity, and sorptivity. BC plays a significant role in the cycling of organic contaminants, as well as the atmospheric heat balance ${ }^{30,31}$, and CNTs may exert a similar influence on these processes. Several studies suggest that CNTs, like BC, are highly sorptive ${ }^{32}$, although few of these reports account for unexpected sources or losses of sorbates due to CNT impurities (e.g., native PAHs desorbing from CNTs or CNT-associated metals acting to degrade amended sorbates). In addition, none of these investigations consider the effect of experimentally induced CNT surface functionality (e.g., many samples are sonicated to improve CNTwater interaction, and sonication can shorten CNTs and add oxygen-containing functional groups to the CNT surface). While there are no explicit research efforts aimed at determining the $\mathrm{CNT}$ contribution to the atmospheric heat balance, $\mathrm{BC}$ and CNTs have similar light absorbing properties and are assumed to have comparable atmospheric lifetimes. The latter supposition needs to be evaluated, but it is reasonable to expect that $\mathrm{BC}$ soot and CNTs will be subject to similar transport and natural modification processes, especially considering the hydrophobicity, tendency to agglomerate, and surface chemistry of the two materials. Until there is a better understanding of transformation and transport phenomena that are unique to CNTs, initial models of CNT partitioning in the environment can be constructed assuming that engineered nanomaterial (i.e., CNTs) and the natural nanomaterial (i.e., BC) will have similar environmental fates. 

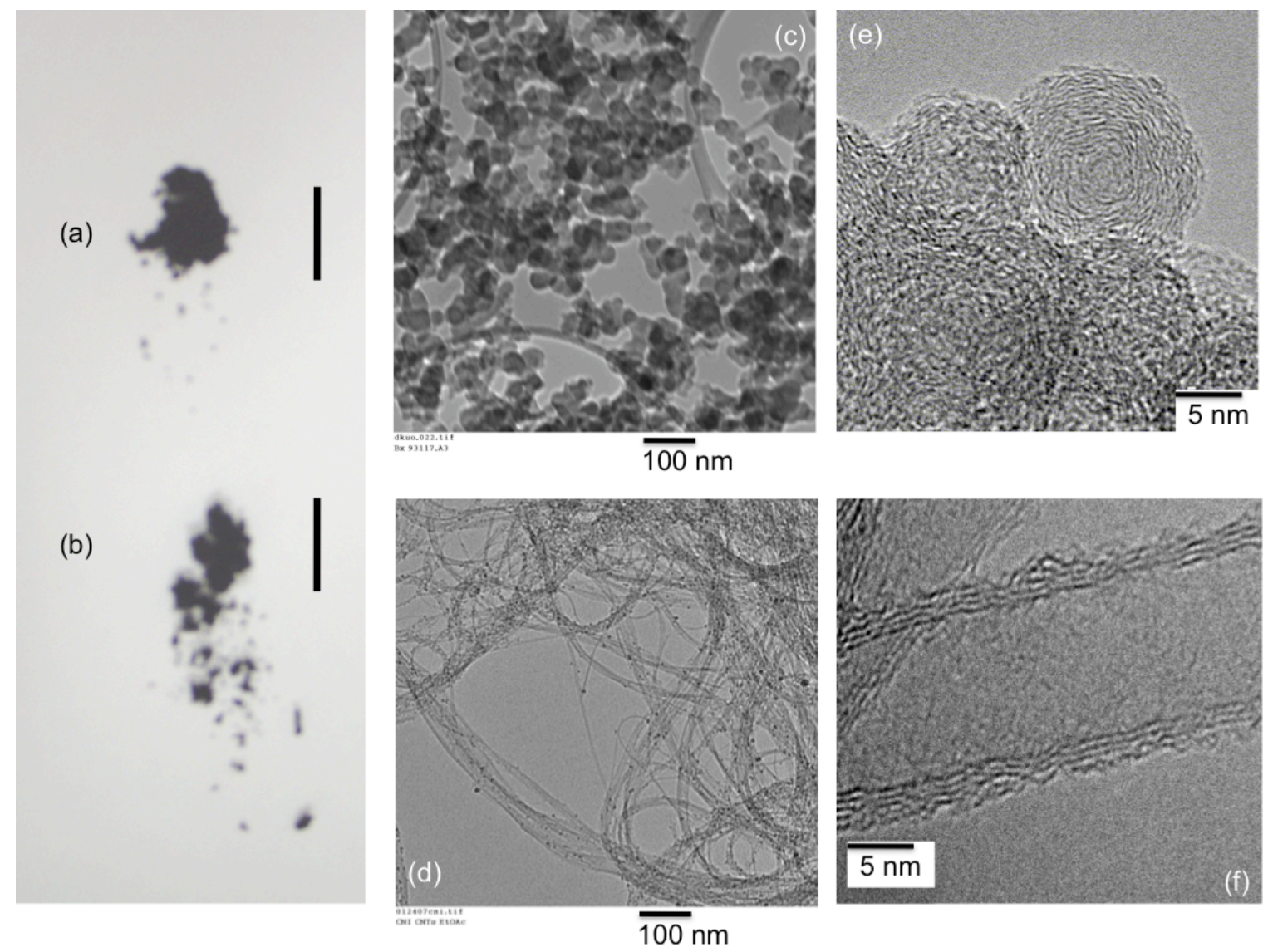

Figure 2. Photographs of (a) black carbon soot (BC soot, National Institute of Standards and Technology Standard Reference Material 1650, Diesel Particulate Matter) and (b) SWCNTs produced by Carbon Nanotech, Inc. The black scale bars are roughly $1.5 \mathrm{~cm}$. Transmission electron micrographs (TEM) of (c) BC soot and (d) SWCNTs (same materials pictured in (a) and (b), respectively). The black scale bars are $100 \mathrm{~nm}$. Highresolution TEM images of (e) BC soot, showing the "onion-like" structure contained in the concentric spheres and (f) MWCNTs, where the multiple walls of the concentric nanotubes are visible. Both scale bars are $5 \mathrm{~nm}$. (Images are courtesy of (c) David Kuo, (e) http://www.ems.psu.edu/\%7Eboehman/flame_materials.html, and (f) Matteo Seita). 


\section{ESTIMATED ENVIRONMENTAL CONCENTRATIONS OF CNTS}

Since the discovery of buckminsterfullerenes in 1985 at Rice University in Houston, Texas, Houston has become the center for fullerene and nanotube research. There are four CNT facilities within Houston's city limits (compared to 7 total facilities in the remainder of the country), and at least two ${ }^{c}$ of those facilities employ CVD to produce CNTs (specifically, SWCNTs). Each facility is capable of producing $45 \mathrm{~kg}$ of bulk product per day (roughly $16,500 \mathrm{~kg}$ annually), and assuming that the product is $30 \%$ CNT-C and the process is $2 \%$ efficient, then CVD effluents in the metropolitan area could reach roughly $4.8 \times 10^{8} \mathrm{~g} \mathrm{C}$ annually. Assuming that CNTs could be released at $0.15 \%$ of the production mass and neglecting post-fabrication release of CNTs, current CNT production volumes would correspond to a particle release of $5 \times 10^{4} \mathrm{~g}_{\text {year }}{ }^{-1}$. Emitted CNTs and BC could travel thousands of kilometers before settling out of the atmosphere (given an average lifetime of 5.3 days for $\mathrm{BC}$ in the atmosphere ${ }^{33}$ and an average windspeed ${ }^{34}$ of $5.7 \mathrm{~m} \mathrm{~s}^{-1}$ ), potentially reaching the Pacific Ocean. Houston is located on the southeastern coast of Texas, adjacent to Galveston Bay in the northwestern corner of the Gulf of Mexico. Given the proximity to CNT point sources, I estimated CNT levels to be on the order of $4.4 \mathrm{pg} \mathrm{m}^{-3}$ in Houston air, $0.2 \mathrm{pg} \mathrm{L}^{-1}$ in Galveston Bay, and $2.0 \mathrm{pg} \mathrm{g}^{-1}$ in Galveston Bay sediment (Figure 3. This model assumed no CNT transformation or degradation reactions in the environment. See Supporting Information for calculations and additional assumptions). At present, CNTs are a trace analyte and any detection method developed for CNTs in the environment will need to be extremely sensitive.

\footnotetext{
${ }^{c}$ One of these facilities, Unidym (formerly Carbon Nanotech Inc.) may stop production in Houston in the near future and relocate to California.
} 


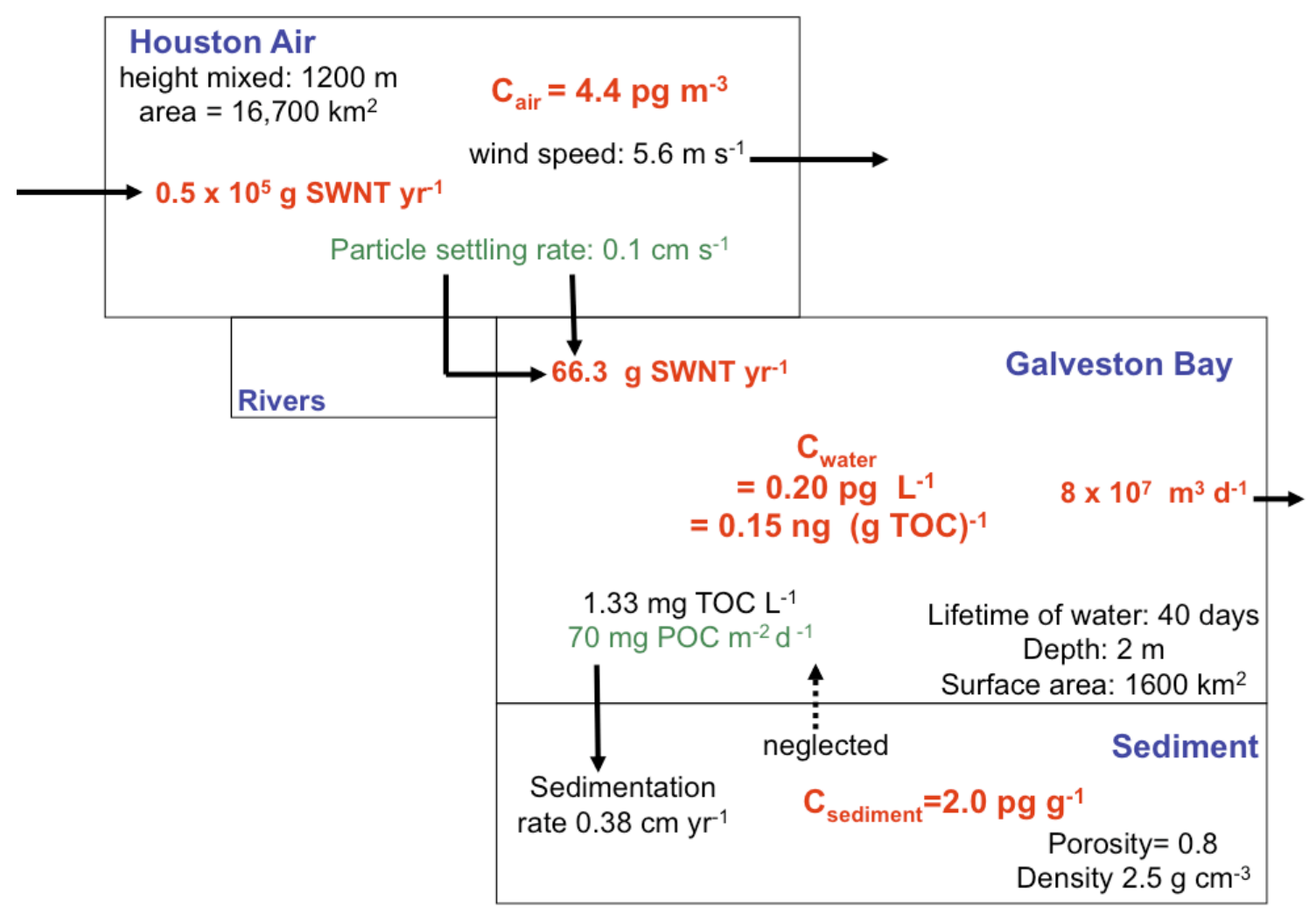

Figure 3. Estimated SWCNT concentrations in Galveston Bay and Houston, TX based on a dynamic mass balance model. The assumptions, calculations, and references for this box model are given in the Supporting Information. Values in black are from the literature, values in green are estimated, and values in red are calculated. 


\section{THESIS OVERVIEW}

In our history, no industrially important chemical has been produced, used, and disposed of without some release to the environment. Furthermore, chemical manufacturing procedures are often designed to maximize material performance and minimize production costs, with little attention devoted to environmental impact mitigation. This model results in postponed recognition of malignant environmental and public health effects, and at an advanced stage of industrial development, it is very difficult to arrest, let alone rectify, the resultant damages. Aware of this pattern and poised at the expansion of an industry, engineers and environmental scientists have a unique opportunity to alter the future approach to the development of all materials: by incorporating environmental objectives prior to industrial scale-up of synthesis. In doing so, we will not only prevent unnecessary harm to the earth and human health, but will also preserve the public's confidence in a field that promises many benefits to society. When this work began in the fall of 2005 , there was no established method for detecting CNTs in complex sample matrices (e.g., air, water, sediments). This limitation precludes both the study of CNT transformation and transport in the environment and limits the ability of government agencies to enforce regulation or gauge public and ecological exposure to the materials. A large portion of this dissertation research was dedicated to overcoming this challenge. First, I investigated the composition of commercially available CNTs to determine (1) the level of diversity in CNTs that might be released to the environment and (2) if there were any characteristics that could be used distinguish CNTs from other organic matter in natural samples (Chapter 2). I then hypothesized that thermal analyses could provide an accessible, cost-effective, high-throughput, minimumsample-handling technique to isolate and quantify CNTs in the environment. This method was developed using thermal gravimetric analysis-mass spectrometry (TGAMS), and while it is not yet sensitive enough for current environmental CNT levels (pg $\mathrm{m}^{-3}$ of air, $\mathrm{pg} \mathrm{L}^{-1}$ of water, $\mathrm{pg} \mathrm{g}^{-1}$ of sediment) it is sufficient for detection of CNTs in production facility air (ug $\mathrm{m}^{-3}$ of air (Chapter 3$)$ ) and can distinguish between BC soot and CNTs. These methods will enable industry and regulators to place upper limits on 
potential emissions from factories and, with further development, will facilitate study of CNTs in the atmosphere, sediments, and ocean.

The environmental impact of CNT production is not limited to the release of CNTs, but includes the release and cycling of carbonaceous co-products. The industrially favored production method, carbon vapor deposition (CVD), is plagued by inefficiency. Less than $3 \%$ of the source carbon feedstock becomes CNT product, while the remaining $\sim 97 \%$ is typically vented to the atmosphere. This material was completely uncharacterized prior to my dissertation research, but I hypothesized that the reaction conditions (heating carbonaceous gas to high temperatures, $500-1200{ }^{\circ} \mathrm{C}$ ) would result in the formation of VOCs, PAHs, and soot, all of which raise environmental and public heath concern. Using a representative CVD reactor, I developed a collection apparatus that enabled detection of some compounds at parts per trillion levels; six orders of magnitude lower than previously possible for these systems. My results showed, as hypothesized, that VOCs, PAHs, soot, and the potent greenhouse gas, methane, were formed from an ethene-fed, laboratory-scale synthetic process and released at unacceptable quantities (Chapter 4). Furthermore, by monitoring the in situ kinetics of CNT formation, I was able to identify which of the thermally generated products were important for CNT growth. Using this information, I successfully delivered the critical CNT precursors without thermal heating, thereby limiting the formation of unwanted byproducts and eliminating the most energetically expensive component of production (Chapter 5).

These results have been and/or will be shared with CNT manufacturers, so that they may make (1) accurate measurements of CNTs and by-products released from their facilities and (2) informed decisions toward environmentally sound production practices as their businesses continue to grow. At this critical juncture, there must be an understanding of the potential environmental impacts of these promising materials, so that we may tailor fabrication procedures and product handling in such a way so as to protect public health, as well as the health of the Earth System. 


\section{REFERENCES}

[1] Girifalco, L.A.; Hodak, M.; Lee, R.S. Carbon nanotubes, buckyballs, ropes, and a universal graphitic potential. Phys. Rev. B 2000, 62, 12104-13110.

[2] de Heer, W.A. Nanotubes and the pursuit of applications. MRS Bulletin. April 2004, 281-285.

[3] http://www.eastonbike.com/PRODUCTS/TECHNOLOGY/tech_cnt.html; Accessed 10 Sept 2005 and 04 April 2009.

[4] www.fibrils.com; Accessed 10 Sept 2005 and 04 April 2009.

[5] Thayer, A.M. Carbon nanotubes by the metric ton. Chemical \& Engineering News 2007, 85 (46), 29-35.

[6] Schwarzenbach, R.P.; Gschwend, P.M.; Imboden, D.M. Environmental Organic Chemistry, $2^{\text {nd }}$ Edition. 2003 New York, NY. John Wiley and Sons. pp. 4-8.

[7] Ball, P. Roll up for the revolution. Nature 2001, 414, 142-144.

[8] Wiles, P.G.; Abrahamson, J. Carbon fibre layers on arc electrodes-I: Their properties and cool-down behaviour. Carbon. 1978, 16 (5), 301-405.

[9] Abrahamson, J.; Wiles, P.G.; Rhoades, B.L. "Structure of carbon fibres found on carbon arc anodes." Abstract in proceedings of the $14^{\text {th }}$ Biennial Conference on Carbon, Pennsylvania Statue University, June 1979. Reprinted in Carbon 1999, 37 (11), 1873-1874.

[10] Iijima, S. Helical microtubules of graphitic carbon. Nature 1991, 354, 56-58.

[11] Kong, J.; Cassell, A.M.; Dai, H. Chemical vapor deposition of methane for single-walled carbon nanotubes. Chem. Phys. Lett. 1998, 292, 567-574.

[12] Nikolaev, P.; Bronikowski, M.J.; Bradley, R.K.; Rohmund, F.; Colvert, D.T.; Smith, K.A.; Smalley, R.E. Gas-phase catalytic growth of single-walled carbon nanotubes from carbon monoxide. Chem. Phys. Lett. 1999, 313, 9197.

[13] Ding, R.G.; Lu, G.Q.; Yan, Z.F.; Wilson, M.A. Recent advances in the preparation and utilization of carbon nanotubes for hydrogen storage. $J$. Nanosci. Nanotechnol. 2001, 1(1), 7-29. 
[14] Plata, D.L.; Gschwend, P.M.; Reddy, C.M. Industrially synthesized singlewalled carbon nanotubes: compositional data for users, environmental risk assessments, and source apportionment. Nanotechnology 2008, 19, 185706.

[15] Eklund, P.C.; Ajayan, P.M.; Blackmon, R.; Hart, A.J.; Kong, J.; Pradhan, B.; Rao, A.; Rinzler, A.G. International Assessment of Research and Development on Carbon Nanotubes: Manufacturing and Applications, World Technology Evaluation Center Report, 2008, http://www.wtec.org/cnm.

[16] Healy, M.L.; Dahlben, L.J.; Isaacs, J.A. Environmental assessment of singlewalled carbon nanotube processes. J. Industrial Ecology 2008, 12 (3), 376393.

[17] Plata, D.L.; Hart, A.J.; Reddy,C.M.; Gschwend, P.M. Early evaluation of potential environmental impacts of carbon nanotube synthesis by catalytic chemical vapor deposition. Chapter 4 in MIT Thesis. June 2009.

[18] National Academy of Sciences News Release, "Federal research plan inadequate to shed light on health and environmental risks posed by nanomaterials." Dec 10, 2008.

[19] Web of Science ${ }^{\mathrm{TM}} \&$ PubMed $^{\mathrm{TM}}$ searches; 15 January 2009.

[20] Lam, C-W.; James, J.T.; McCluskey, R.; Hunter, R.L. Pulmonary toxicity of single-wall carbon nanotube in mice $7 \& 90$ days after intratracheal instillation. Toxicol. Sciences. 2004, 77, 126-134.

[21] Jia, G.; Wang, H.; Yan, L.; Wang, X.; Pei, R.; Yan, T.; Zhao, Y.; Guo, X. Cytotoxicity of carbon nanomaterials: single-wall nanotube, multi-wall nanotube, and fullerene. Environ. Sci. Technol. 2005, 39, 1378-1383.

[22] Poland, C.A.; Duffin, R.; Kinloch, I.; Maynard, A.; Wallace, W.A.H.; Seaton, A.; Stone, V.; Brown, S.; MacNee, W.; Donaldson, K. Carbon nanotubes introduced intot he abdominal cavity of mice show asbestoslike pathogenicity in a pilot study. Nature Nanotechnology 2008, 3, 423-428.

[23] Templeton, R.C.; Ferguson, P.L.; Washburn, K.M.; Scrivens, W.A.; and Chandler, G.T. Life-cycle effects of single-walled carbon nanotubes (CNTs) on an estuarine meiobenthic copepod. Environ. Sci. Technol. 2006, 40 (23), 7387-7393. 
[24] Guo, L.; Morris, D.G.; Liu, X.; Vaslet, C.; Hurt, R.H.; Kane, A.B. Iron bioavailability and redox ativity in diverse carbon nanotube samples. Chem. Mater. 2007, 19 (14), 3472-3478.

[25] Kang, S.; Mauter, M.S.; Elimelech, M. Physicochemical determinants of multiwalled carbon nanotube bacterial cytotoxicity. Environ. Sci. Technol. 2008, 42, 7528-7534.

[26] Shi Kam, N.W.; Jessop, T.C.; Wender, P.A.; Dai, H. Nanotube molecular transporters: internalization of carbon nanotube-porotein conjugates into mammalian cells. J. Am. Chem. Soc. 2004, 126(22), 6850-6851.

[27] Murr, L.E.; Soto, K.F.; Esquivel, E.V.; Bang, J.J.; Guerrero, P.A.; Lopez, D.A.; Ramirez, D.A. Carbon nanotubes and other fullerene-related nanocrystals in the environment: A TEM Study. J. Minerals, Metals, \& Materials Society. June 2004. 28-31.

[28] Murr, L.E.; Esquivel, E.V.; Bang, J.J.; de la Rosa, G.; Gardea-Torresday, J.L. Chemistry and nanoparticulate compositions of a 10,000 year-old ice core melt water. Water Research. 2004, 38, 4282-4296.

[29] Schmidt, M.W.I.; Noack, A.G. Black carbon in soils and sediments: Analysis, distribution, implications, and current challenges. Global Biogeochemical Cycles 2000, 14 (3), 777-793.

[30] Hammes, K.; et 32 co-authors. Comparison of quantification methods to measure fire-derived (black/elemental) carbon in soils and sediments using reference materials from soil, water, sediment and the atmosphere. Global Biogeochemical Cycles 2007, 21, GB3016.

[31] Gustafsson, Ö.; Haghseta, F.; Chan,C.; MacFarlane, J.; Gschwend, P.M. Quantification of the dilute sedimentary soot phase: implications for PAH speciation and bioavailability. Environ. Sci. Technol. 1997, 31, 203-209.

[32] Yang, K.; Zhu, L.; Xing, B. Adsorption of polycyclic aromatic hydrocarbons by carbon nanomaterials. Environ. Sci. Technol. 2006, 40, 1855-1861.

[33] Cooke, W.F., Liousse, C.; Cachier, H. Construction of a $1^{\circ} \mathrm{X} 1^{\circ}$ fossil fuel emission data set for carbonaceous aerosol and implementation and radiative impact in the ECHAM4 model. J. Geophysical Research. 1999 104(D18), 22137-22162. 
[34] National Oceanic and Atmospheric Administration. National Data Buoy Center. Buoy IDs: 42035, 42043. Archive windspeed data for 2004. (http://www.ndbc.noaa.gov/Maps/WestGulf.shtml) 


\section{SUPPORTING INFORMATION}

\section{Box Model Calculations \& Assumptions}

For Houston air: This expression accounts for CNT input from production facilities and advection into Houston from other areas, and CNT loss due to advection out of Houston and dry deposition settling from the air column. Symbols, references, and assumptions are given in Table 1 .

$$
V \frac{d C_{\text {air }}}{d t}=R_{\text {in }}+C_{\text {air }, \text { in }}\left(v_{\text {wind }} A_{\text {Houston }}\right)-C_{\text {air }}\left(v_{\text {wind }} A_{\text {Houston }}\right)-C_{\text {air }} f_{\text {settling }}
$$

Assuming steady-state and solving equation 1 for $\mathrm{C}_{\mathrm{air}}$ :

$$
C_{\text {air }}=\frac{R_{\text {in }}+C_{\text {air }, \text { in }}\left(v_{\text {wind }} A_{\text {Houston }}\right)}{\left(v_{\text {wind }} A_{\text {Houston }}\right)+f_{\text {settling }}}
$$


Table S1. Information used to calculate $\mathrm{C}_{\text {air }}$ in Houston, TX.

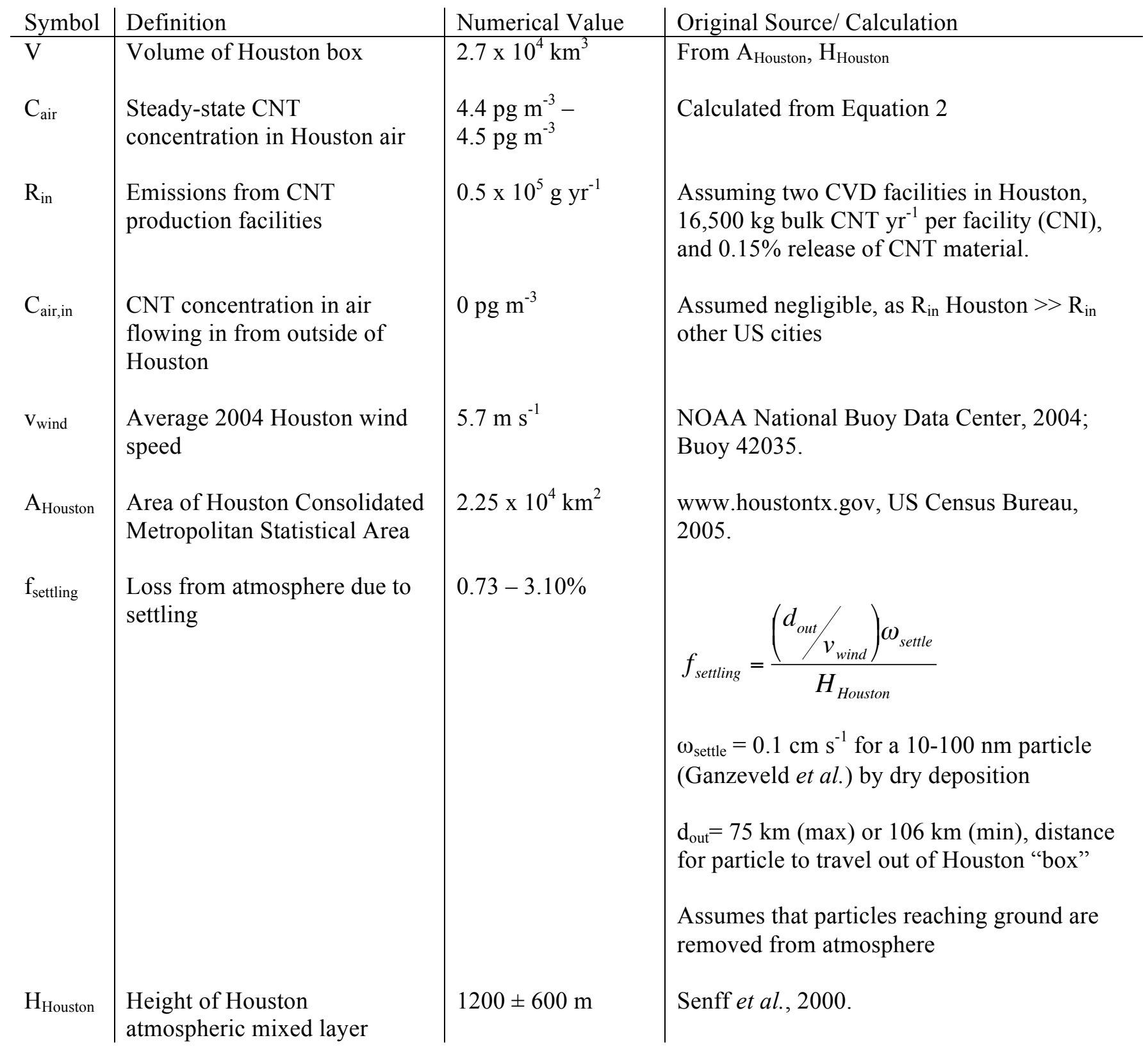


For Galveston Bay water: This expression accounts for CNT input from the air and river (as one term), and CNT loss due to advection out of Galveston Bay and settling from the water column with organic carbon (see Table 2 for definition of terms).

Symbols, references, and assumptions are given in Table 2.

$$
V_{b a y} \frac{d C_{\text {water }}}{d t}=Q_{i n}-C_{\text {water }} F_{T O C} A_{b a y}-C_{\text {water }} C_{T O C} \frac{V_{\text {bay }}}{\tau_{\text {water }}}
$$

Assuming steady-state and solving equation 3 for $\mathrm{C}_{\mathrm{water}}$ :

$$
C_{\text {water }}=\frac{Q_{\text {in }}}{F_{T O C} A_{b a y}-C_{T O C} \frac{V_{b a y}}{\tau_{\text {water }}}}
$$

\begin{tabular}{|c|c|c|c|}
\hline fyambol & Diffitinimo of water in bay & Nungersical Value & 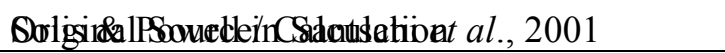 \\
\hline $\begin{array}{l}\mathrm{V}_{\text {bay }} \\
\mathrm{H}_{\text {bay }}\end{array}$ & $\begin{array}{l}\text { Volume of Galveston Bay } \\
\text { Average depth of bay }\end{array}$ & $\begin{array}{l}3.2 \times 10^{9} \mathrm{~m}^{3} \\
2 \mathrm{~m}\end{array}$ & $\begin{array}{l}\text { From } \mathrm{A}_{\text {bay }}, \mathrm{H}_{\text {bay. }} \\
\text { Santschi et al., } 2001\end{array}$ \\
\hline $\mathrm{C}_{\mathrm{water}}$ & $\begin{array}{l}\text { Steady-state CNT concentration } \\
\text { in Galveston Bay }\end{array}$ & $\begin{array}{l}0.2 \mathrm{pg} \mathrm{L}^{-1}- \\
0.15 \mathrm{ng}(\mathrm{g} \text { TOC })^{-1}\end{array}$ & Calculated from Equation 4 \\
\hline $\mathrm{Q}_{\text {in }}$ & $\begin{array}{l}\text { Atmospheric and riverine } \\
\text { runoff into Galveston Bay }\end{array}$ & $\begin{array}{l}22.1 \mathrm{~g} \mathrm{yr}^{-1}- \\
* 66.3 \mathrm{~g} \mathrm{yr}^{-1}\end{array}$ & $\begin{array}{l}\text { Given } 0.1 \mathrm{~cm} \mathrm{~s}^{-1} \text { settling rate, } \mathrm{H}_{\mathrm{Houston}}, 85 \mathrm{~km} \\
\text { distance from Houston center to beyond } \\
\text { Galveston Bay, winds blow to east/southeast } \\
5.2 \% \text { of time with an average speed of } \\
5.6 \mathrm{~m} \mathrm{~s}^{-1} \text {. Assumes that all of the CNTs that } \\
\text { settle on land, in river, and on the surface of } \\
\text { Galveston Bay, are transferred into Galveston } \\
\text { Bay over the course of the year. } \\
\text { * maximum employed in calculation. }\end{array}$ \\
\hline $\mathrm{F}_{\mathrm{TOC}}$ & $\begin{array}{l}\text { Flux of total organic carbon } \\
\text { (TOC) to sediments }\end{array}$ & $25.55 \mathrm{~g} \mathrm{~m}^{-2} \mathrm{yr}^{-1}$ & $\begin{array}{l}\text { Assumed TOC flux in Galveston Bay similar } \\
\text { to that in Pensacola Bay, FL ( } 70 \mathrm{mg} \mathrm{C} \mathrm{m}^{-2} \mathrm{~d}^{-1} \text {, } \\
\text { Murrell et al., 2001). (Sediment accumulation } \\
\text { rate in Santschi et al. (2001) is } 160 \mathrm{~g} \mathrm{~m}^{-2} \mathrm{yr}^{-1} \text {, } \\
\text { but includes inorganic materials in addition to } \\
\text { TOC). }\end{array}$ \\
\hline $\mathrm{A}_{\text {bay }}$ & Area of Galveston Bay & $1.6 \times 10^{9} \mathrm{~m}^{2}$ & Santschi et al., 2001 \\
\hline $\mathrm{C}_{\mathrm{TOC}}$ & TOC concentration in bay & $1.33 \mathrm{mg} \mathrm{TOC} \mathrm{L}{ }^{-1}$ & 2003. www.galvestonbaydata.org \\
\hline
\end{tabular}

Table S2. Information used to calculate $\mathrm{C}_{\mathrm{water}}$ in Galveston Bay, TX. 
For Galveston Bay sediment: Assuming steady-state, where CNT flux to the sediment-water interface is equal to the $\mathrm{CNT}$ burial, then we can solve for $\mathrm{C}_{\text {sed: }}$ :

$$
C_{\text {sed }}=\frac{\omega_{\text {sed }} \rho(1-\Phi)}{C_{\text {water }} F_{T O C}}
$$

Symbols, references, and assumptions are given in Table 3.

Table S3. Information used to calculate $\mathrm{C}_{\text {sed }}$ in Galveston Bay, TX.

\begin{tabular}{l|l|l|l} 
Symbol & Definition & Numerical Value & Original Source/ Calculation \\
\hline $\mathrm{C}_{\text {sed }}$ & $\begin{array}{l}\text { Steady-state CNT concentration } \\
\text { in Galveston Bay sediments }\end{array}$ & $2.0{\mathrm{pg}(\mathrm{g} \text { sediment })^{-1}}$ & Calculated from Equation 5 \\
$\omega_{\text {sed }}$ & $\begin{array}{l}\text { Sedimentation rate } \\
\rho\end{array}$ & $0.38 \mathrm{~cm} \mathrm{yr}^{-1}$ & Santschi et al., 2001 \\
$\Phi$ & Density, bulk sediment & $2.5 \mathrm{~g} \mathrm{~cm}^{-3}$ & Santschi et al., 2001 \\
& Sediment porosity & 0.8 & Santschi et al., 2001
\end{tabular}

\section{REFERENCES}

[1] Ding, R.G.; Lu, G.Q.; Yan, Z.F.; Wilson, M.A. Recent advances in the preparation and utilization of carbon nanotubes for hydrogen storage. $J$. Nanosci. Nanotechnol. 2001, 1(1), 7-29.

[2] Kong, J.; MIT; Personal communication, September 2005.

[3] National Oceanic and Atmospheric Administration. National Data Buoy Center. Buoy IDs: 42035, 42043. Archive windspeed data for 2004. (http://www.ndbc.noaa.gov/Maps/WestGulf.shtml)

[4] Santschi, P.H.; Presley, B.J.; Wade, T.L.; Garcia-Romero, B.; Baskaran, M. Historical contamination of PAHs, PCBs, DDTs, and heavy metals in Mississippi River Delta, Galveston Bay and Tampa Bay sediment cores. Marine Envrion. Research. 2001, 52, 51-79.

[5] U.S. Census Bureau. http://quickfacts.census.gov/qfd/. Accessed on Sept 01, 2005.

[6] U.S. Environmental Protection Agency. AP 42, Vol. 1, Fifth Edition. Particle size distribution data and sized emission factors for selected sources. 1995. 
[7] Murell, M.C.; Hagy, J.D.; Greene, R.M.; James, J.B. Phytoplankton- and detritus-based food webs in Gulf of Mexico estuaries: Lessons from Pensacola Bay, FL, USA. Estuarine Research Federation. 2001 Conference.

[8] Senff, C.; Banta, R.; Darby, L.; Angevine, W.; White, A.; Berkowitz, C.; Doran, C. Spatial and temporal variations in mixing height in Houston TNRCC project F-20, Final Report for NOAA. 2000. 


\section{CHAPTER 2: \\ INDUSTRIALLY SYNTHESIZED SINGLE-WALLED CARBON NANOTUBES: COMPOSITIONAL DATA FOR USERS, ENVIRONMENTAL RISK ASSESSMENTS, AND SOURCE APPORTIONMENT}

\section{By}

Desirée L. Plata $^{1,2}$, Philip M. Gschwend ${ }^{1}$, and Christopher M. Reddy ${ }^{2}$

${ }^{1}$ Department of Civil and Environmental Engineering, Massachusetts Institute of Technology, Cambridge, MA 02139, USA

${ }^{2}$ Department of Marine Chemistry and Geochemistry, Woods Hole Oceanographic Institution, Woods Hole, MA 02543, USA

This chapter originally appeared as a manuscript in:

Nanotechnology 19, 2008, 185706 (14 pp)

It is reproduced here with copyright permission of the Institute of Physics.

Their website can be accessed at www.iop.org/journals/nano.

An electronic abstract to this article is available at

http://www.iop.org/EJ/abstract/-search=62564756.1/0957-4484/19/18/185706. 


\begin{abstract}
Commercially available single-walled carbon nanotubes (SWCNTs) contain large percentages of metal and carbonaceous impurities. These fractions influence SWCNT physical properties and performance, yet their chemical compositions are not well defined. This lack of information also precludes accurate environmental risk assessments for specific SWCNT stocks, which emerging local legislation requires of nanomaterial manufacturers. To address these needs, we measured the elemental, molecular, and stable carbon isotope compositions of commercially available SWCNTs. As expected, catalytic metals occurred at percent levels, but purified materials also contained unexpected metals (e.g., $\mathrm{Cu}, \mathrm{Pb}$ ). Nitrogen contents (up to $0.48 \%$ ) were typically greater in arc-produced SWCNTs than those derived from chemical vapor deposition. Tolueneextractable materials contributed less than $5 \%$ of the total mass of the SWCNTs. Internal standard losses during dichloromethane extractions suggested that metals are available for reductive dehalogenation reactions, ultimately resulting in the degradation of aromatic internal standards. The carbon isotope content of the extracted compounds suggested that SWCNTs acquired much of their carbonaceous contamination from their storage environment. Some of the SWCNTs, themselves, were highly depleted in ${ }^{13} \mathrm{C}$ relative to petroleum-derived chemicals. The distinct carbon isotopic signatures and unique metal "fingerprints" may be useful as environmental tracers allowing assessment of SWCNT sources to the environment.
\end{abstract}

\title{
INTRODUCTION
}

Single-walled carbon nanotubes (SWCNTs) are promising new materials noted for their exceptional electrical properties, mechanical strength, and thermal stability. Due to their unique characteristics and novel synthetic methods, there is a great deal of uncertainty surrounding the possible influence of SWCNTs on the environment [1] and human health [2]. Previous efforts to assess the risks associated with the industry have considered production reagents and energy use [3], but there is a distinct lack of information regarding the environmental influence of the produced nanomaterials themselves. Two recent reports $[1,4]$ highlighted the need for such analyses, outlining challenges to the 
safe advancement of the SWCNT industry. Explicitly, the authors call for the development of (1) methods to assess the environmental fate of engineered nanomaterials and (2) nanomaterial-specific toxicity data for dependable risk assessment modeling. Wiesner et al. [1] emphasize that surface chemistry and morphology may influence the toxicity and environmental mobility of a given nanoparticle.

SWCNTs, which are formed from a variety of synthetic techniques, will have variable chemistries and physical properties resulting from their different metal catalysts or amorphous carbon coatings [5]. The latter can have environmental and toxicological impacts. For example, fluorescent fractions of the amorphous carbon associated with arcproduced SWCNTs impaired the development of estuarine copepods, whereas purified materials showed no remarkable toxicity [6]. Preliminary studies indicate no clear relationship between SWCNT metal content and respiratory impairment $[7,8]$, however, the metal composition may dictate the environmental transport of, and ultimate biological exposure to, SWCNTs. For instance, oxides of the selected catalyst could confer charge on the nanomaterials. As surface charges control the aggregation of submicron-sized particles [9], SWCNTs with different metal catalysts may exhibit differing environmental lifetimes in air and water. Additionally, associated impurities could control SWCNT participation in photochemical processes and interactions with biological membranes [2, $10,11]$. Metal catalyst and amorphous carbon impurities can account for up to $70 \%$ of a bulk SWCNT material [12-14], yet these fractions are largely uncharacterized. Thus, our ignorance of the diversity of compositions of SWCNTs prohibits a mechanistic understanding of SWCNT electronic character, environmental transport, transformation, and ecotoxicology. Consequently, no predictive risk assessment models can be constructed at this time. Maynard et al. [4] warn that this lack information could result in eroded public confidence through real or apparent dangers, impacting market growth during this critical time of expansion, as well as unintended environmental and public health consequences.

To begin to rectify this situation, we determined the metal, carbon, nitrogen, stable carbon isotopic, and solvent-extractable aromatic hydrocarbon content of SWCNTs produced by ten companies in the United States (US) (Table 1). Our specific goals were to (1) identify metal catalysts and aromatic hydrocarbons that would be released with and 
affect the properties of SWCNTs, (2) seek compositional data suited to pursuing environmental exposure modeling of SWCNTs, and (3) find properties that would be helpful for detecting, and perhaps apportioning sources of, SWCNTs in environmental matrices. By pursuing these objectives while the SWCNT industry is developing largecapacity facilities, we seek to provide feedback to the manufacturers so as to avoid human health and environmental damage. 


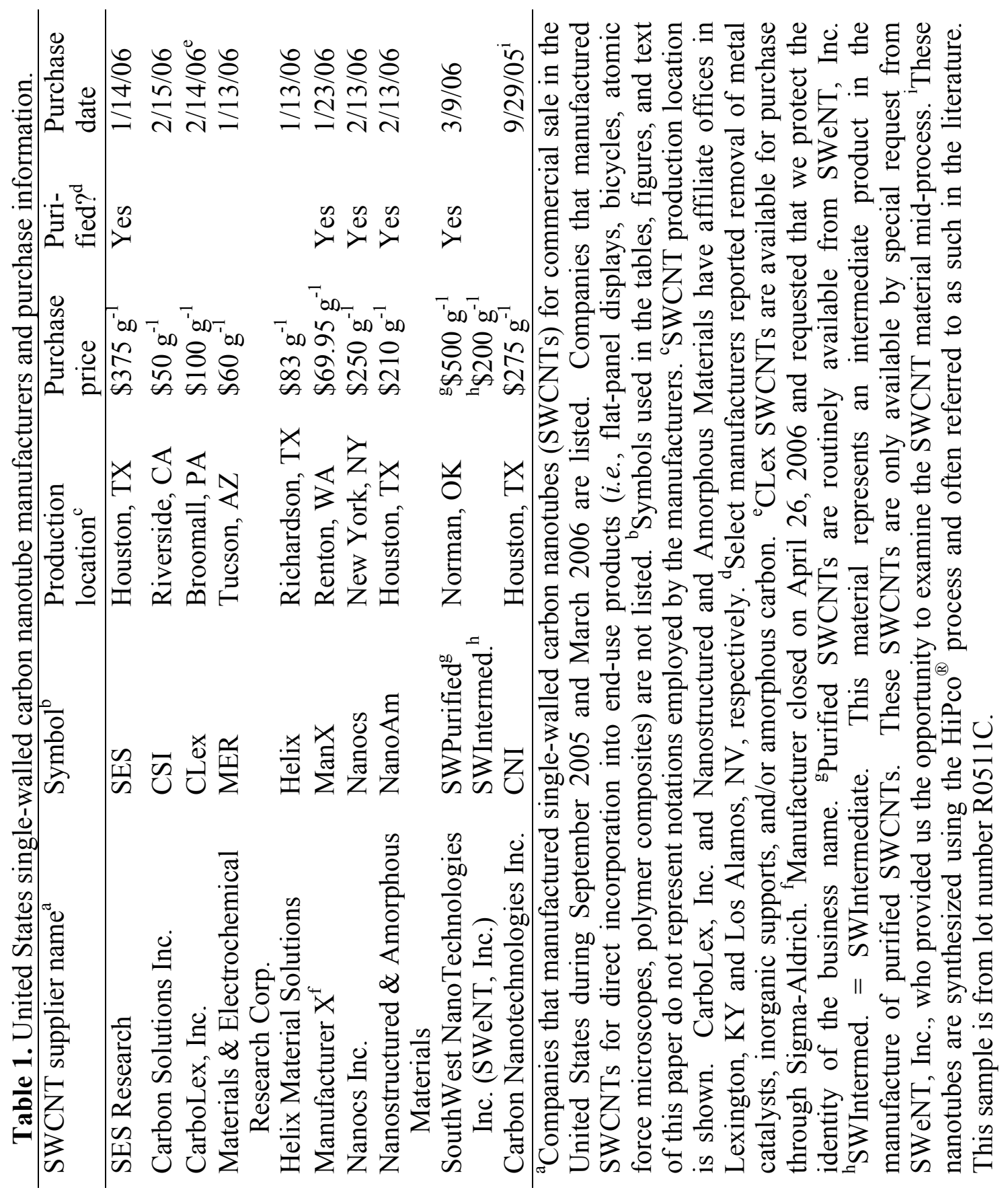




\section{EXPERIMENTAL METHODS}

Samples. SWCNTs were purchased from all known, US-based manufacturers between September 2005 and March 2006 (Table 1). Advantageously, the materials represent a variety of fabrication techniques and include both purified and unpurified SWCNTs. As-produced SWCNTs (AP-SWCNTs) contain metal catalyst and amorphous carbon impurities and range in price from $\$ 50$ to $\$ 275 \mathrm{~g}^{-1}$. Purified SWCNTs are thermally and/or chemically treated and cost between $\$ 70$ and $\$ 500 \mathrm{~g}^{-1}$. To compare the differences in purified and unpurified SWCNTs, we analyzed nanotubes from one manufacturer, SWeNT, Inc., before (SWIntermediate) and after purification (SWPurified) (Table 1).

Metal, metalloid, and rare earth element analysis. Plastic and quartz ware were cleaned by soaking for 2 weeks in $3 \mathrm{M}$ hydrochloric acid $(\mathrm{HCl})$ and 2 days in $8 \mathrm{M}$ nitric acid $\left(\mathrm{HNO}_{3}\right)$ at room temperature (acids were Fisher Trace Metal Grade). SWCNTs were delivered to $10-\mathrm{mL}$ quartz (Type IV) beakers in ethyl acetate (EtOAc) suspensions (see Supporting Information), evaporated to dryness, and combusted for $6 \mathrm{hr}$ at $850^{\circ} \mathrm{C}$. Samples were then digested by refluxing in $1 \mathrm{~mL} 16 \mathrm{M} \mathrm{HNO}_{3}$ (Fisher Optima) for 30 min, evaporated to dryness, dissolved in $3 \mathrm{~mL} 12 \mathrm{M} \mathrm{HCl}$ (Fisher Optima), evaporated to dryness again, and finally re-dissolved with $1.2 \mathrm{M} \mathrm{HCl}$. Solutions were filtered through $0.2 \mathrm{~mm}$ filters and analyzed in quadruplicate (duplicate measurement of two distinct dilutions) by inductively coupled plasma-mass spectrometry (ICP-MS). Samples were analyzed for Al, Ag, Au, Ba, Be, Bi, By, Ca, Ce, Cd, Co, Cr, Cs, Cu, Er, Fe, Ga, Gd, Hf, Ho, Hg, Ir, La, Li, Lu, Mo, Nb, Nd, Ni, Os, Pb, Pr, Pt, Re, Rh, Ru, Sb, Sm, Sn, Sr, Ta, Tb, Te, Th, Ti, Tm, U, V, W, Y, Yb, Zr. Only elements present above the detection limit are reported here. Detection limits (all in ppm) were 100 (CNI), 50 (Helix, Nanocs), 20 (CLex, CSI, MER, ManX, SES, SWPurified, SWIntermediate), and 7 (NanoAm), varying between samples due to differences in SWCNT sample size and consistent between elements because the maximum detection limit for any one element is reported.

Silica was measured in two samples thought to contain $\mathrm{SiO}_{2}$ as a growth substrate (SWPurified and SWIntermediate). Briefly, samples (5-10 mg) and $\mathrm{LiBO}_{3}(\sim 70 \mathrm{mg})$ were added to graphite crucibles and heated at $950^{\circ} \mathrm{C}$ for $15 \mathrm{~min}$ [15]. The hot liquid was 
poured into a Teflon beaker, stirred with $10 \mathrm{~mL}$ of $0.8 \mathrm{M} \mathrm{HNO}_{3}$ for $15 \mathrm{~min}$, transferred to a tared plastic vial, and diluted with milli-Q water to a final mass of $20 \mathrm{~g}$. After filtration though a $0.45 \mathrm{~mm}$ filter, solutions were analyzed by ICP-emission spectroscopy.

Determining bulk carbon and nitrogen content of SWCNTs. SWCNTs were delivered to silver capsules $(15 \times 7.5 \mathrm{~mm})$ in EtOAc suspensions using 1-mL, glass gastight syringes. Syringes were rinsed seven times between sample replicates and were disassembled and sonicated for $20 \mathrm{~min}$ in EtOAc between different SWCNT samples. After drying overnight $\left(60^{\circ} \mathrm{C}\right)$, the residue was analyzed for carbon $(\mathrm{C})$ and nitrogen $(\mathrm{N})$ content using an Elementar CHN Analyzer. Samples were analyzed in triplicate, except for CNI and CSI $(n=6)$.

In order to distinguish SWCNT-C from amorphous $\mathrm{C}$ and other carbonaceous impurities, we performed a temperature-programmed oxidation using thermogravimetric analysis (TGA, TA Instruments Q50). The initial sample mass was 3-3.5 mg, except for SWIntermed., whose initial mass was much larger $(15 \mathrm{mg})$ to compensate for the low expected $\mathrm{C}$ content. Each sample was heated at $5^{\circ} \mathrm{C} \mathrm{min}^{-1}$ to $950{ }^{\circ} \mathrm{C}$ (10 min hold) in dry air. The differentiated thermogram was fit assuming a bimodal Gaussian distribution using a non-linear least squares technique. Co-oxidizing phases can bias the analysis, giving higher-than-actual measured C content. These phases were present in SES (giving an overestimate of amorphous C), CSI (giving an overestimate of SWCNT-C), and MER samples.

EtOAc was used to deliver the SWCNTs to the silver capsules, as it improved precision by reducing sample loss and sample-to-sample contamination due to electrostatic interactions. To confirm that EtOAc did not affect our results, we compared the $\mathrm{C}$ content of the National Institute of Standards and Technology Standard Reference Material 1649a "Urban Dust" delivered with and without EtOAc; both gave values that matched the certified $\mathrm{C}$ content.

Determining stable carbon isotopic content of SWCNTs. Dry SWCNTs were transferred to a 9-mm cylindrical quartz insert, which was then inverted into a $12-\mathrm{mm}$ quartz tube containing roughly $2 \mathrm{~g}$ of cupric oxide. The tubes were evacuated on a vacuum line, flame sealed, and combusted for $5 \mathrm{hr}$ at $850^{\circ} \mathrm{C}$. Carbon dioxide was isolated through a series of cold traps and ${ }^{13} \mathrm{C} /{ }^{12} \mathrm{C}$ was measured by isotope ratio mass 
spectrometry (IRMS) [16]. The ${ }^{13} \mathrm{C} /{ }^{12} \mathrm{C}$ content is reported relative to the ${ }^{13} \mathrm{C} /{ }^{12} \mathrm{C}$ of Vienna Pee Dee Belemnite (VPDB) as

$$
\delta^{13} C_{V P D B}\left({ }^{o} /_{o o}\right)=\left(\frac{\left({ }^{13} C /{ }^{12} C\right)_{\text {sample }}-\left({ }^{13} C /{ }^{12} C\right)_{V P D B}}{\left({ }^{13} C /{ }^{12} C\right)_{V P D B}}\right) x 1000
$$

Each sample was measured in quadruplicate, except for SES, MER, SWIntermed., Helix $(\mathrm{n}=2)$, and CLex $(\mathrm{n}=3)$.

\section{Dichloromethane and toluene extractions}

Extractable polycyclic aromatic hydrocarbons (PAHs). Dry SWCNTs were added to $50-\mathrm{mL}$ glass centrifuge tubes containing $50 \mathrm{~mL}$ of DCM or toluene. To gauge extraction efficiency and sample recovery, $5 \mathrm{ug}$ each of $d_{10}$-acenaphthene, $m$-terphenyl, and $d_{12}$-perylene were added as internal standards. The solutions were then equilibrated over 8 days at room temperature. After a $5 \mathrm{hr}, 2250 \mathrm{rpm}(680 \mathrm{~g})$ centrifugation, $10-\mathrm{mL}$ sub-samples of the extracts were transferred to pear-shaped flasks and concentrated by rotary evaporation. Each extract was spiked with calibration standards $\left(d_{10}\right.$-anthracene, $d_{10}$-fluoranthene, and $d_{12}$-benz $[a]$ anthracene) and analyzed in triplicate by gas chromatography-mass spectrometry (GC-MS). Detection limits (Table 2) vary between SWCNTs due to differences in the initial mass of SWCNTs extracted and reconstructed ion chromatogram noise.

The dichloromethane extraction was performed twice and the toluene extraction was performed once. To ensure the validity of the comparison, data shown here are from toluene and dichloromethane extractions that were performed simultaneously. 


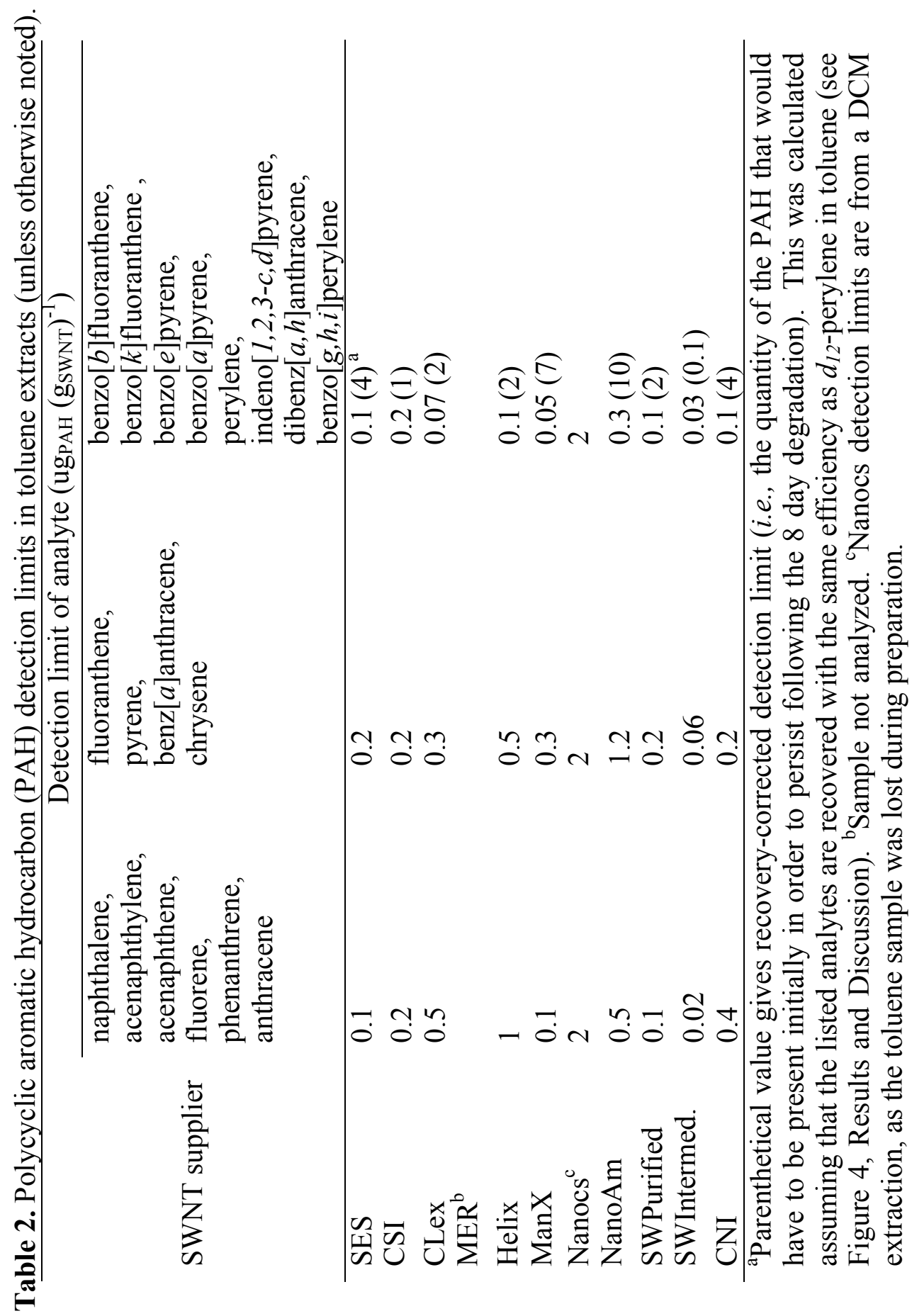


Stable carbon isotope content of extractable carbonaceous material. Another 10-mL sub-sample of each DCM extract was concentrated by rotary evaporation and transferred to a pre-combusted $1.5-\mathrm{mL}$ amber vial. Samples were passively evaporated in a laminar flow hood and re-dissolved in chloroform $\left(\mathrm{CHCl}_{3}\right)$. The ${ }^{13} \mathrm{C}$ content of the nonvolatile material was analyzed in triplicate by moving wire-IRMS (MW-IRMS) [17]. MW-IRMS gives accurate $\mathrm{d}^{13} \mathrm{C}_{\mathrm{VPDB}}$ values on nanogram quantities of material. Briefly, $1 \mathrm{~mL}$ of liquid sample was deposited on a nickel wire, and as the wire moves through a drying oven $\left(60^{\circ} \mathrm{C}\right)$, the solvent evaporates and non-volatile compounds remain on the wire. The sample then combusted and dried, and the resultant $\mathrm{CO}_{2}$ is analyzed by IRMS.

\section{RESULTS AND DISCUSSION}

Metal and metalloid content. Residual metal catalysts are considered the principal components of metallic impurities in SWCNTs, accounting for up to $30 \%$ of the bulk material [12]. Commercial syntheses utilize diverse metals in variable proportions, and there are no purification procedures that successfully eliminate all metals from the SWCNTs. Since the abundance and oxidation state of associated metals can impact the charge carrying capacity of the SWCNTs, their reactivity, as well as their environmental fates and effects, one of our objectives was to identify all the metals and their abundances in commercial SWCNT materials. In addition, we sought to identify unique metal "fingerprints" that might be applied to track SWCNTs in the natural world.

Expected and unexpected metals. Purified SWCNTs contain the reported metal catalysts at low abundances (1.3 to $4.1 \%$ total metal; Table 3 ). Perhaps surprisingly, purified SWCNTs also have unexpected metals $(\mathrm{Cr}, \mathrm{Cu}, \mathrm{Pb})$ at part per thousand levels (0.02 to $0.3 \mathrm{ppt})$. Comparing an intermediate product in SWCNT synthesis with a purified material (SWIntermediate and SWPurified, respectively), SWIntermediate contains $\mathrm{Co}$ and $\mathrm{Mo}$, as expected, while SWPurified has $\mathrm{Fe}, \mathrm{Cu}$, and $\mathrm{Pb}$ in addition to the known catalysts. Acid treatments are common in SWCNT purification procedures intended to leach metals from the bulk materials [12]. We speculate that SWCNTs, damp with residual acid, could come in contact with metallic manufacturing equipment, transferring trace quantities of metals to the SWCNTs. Carbon nanotube-associated 
metals can influence charge transfer capacity [18], and unexpected metals may degrade the electronic performance of the materials. Unpurified SWCNTs contain the metals used as catalysts in their syntheses at weight percentages (14 to 29\% total metal, Table 3). Our results agree with those of Warheit et al. [8] for the CarboLex and CNI samples, and are generally similar to those reported previously for the catalysts [19]. Thus, SWCNTs will act as carriers of these metals to the environment. Such releases may have undesirable environmental consequences, acting as either toxic substances $[2,20]$ or nutrients [21] in niches where these substances are present at low levels. For example, Maynard et al. [22] reported airborne levels of SWCNTs during handling near $50 \mathrm{ug} \mathrm{m}^{-3}$; for nanotubes containing $10 \%$ cobalt catalyst, this would imply 5 ug Co $\mathrm{m}^{-3}$, an exposure that is within a factor of 10 of OSHA's time-weighted average exposure limit. Since it seems likely that greater concentrations of SWCNTs would occur in industrial settings [20], toxic exposures to metals like cobalt could result. 


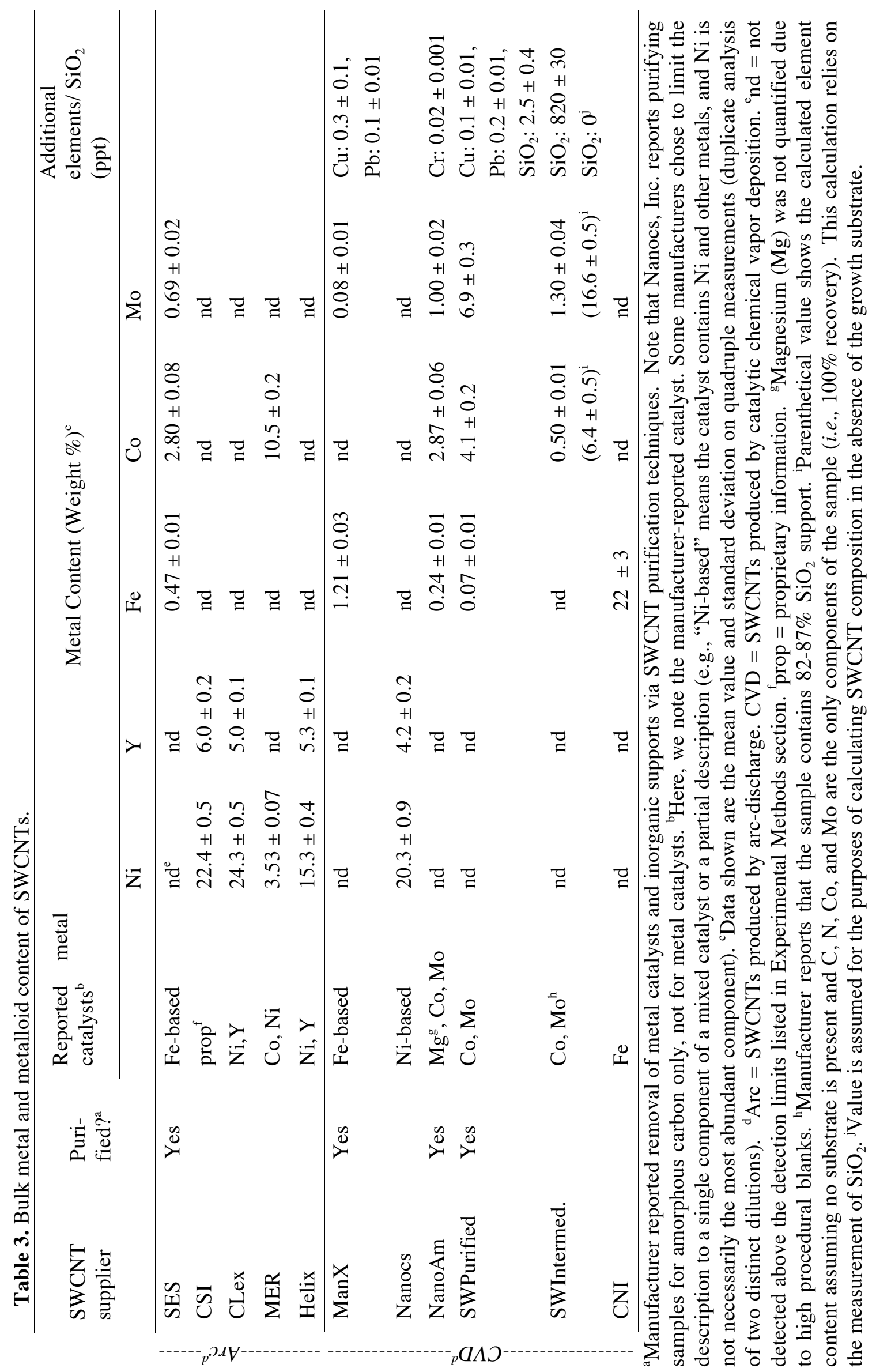


Unique metal ratios for SWCNT source apportionment. While these expected and unexpected metals raise environmental concern, they may be valuable for detecting SWCNTs and apportioning them to diverse sources (both natural and synthetic), as the unique metal composition of an aerosol source can be used to study its transport in the environment [23]. For example, automobile emissions have been traced using platinum group element ratios, which reflect the composition of metal catalysts in catalytic converters $[24,25]$. Given the diversity of metal catalysts used in nanotube synthesis, there is no universal metal "fingerprint" for all SWCNTs. However, we suggest that metal abundances in environmental samples (e.g., aerosol concentrates), in combination with other elements, could be used to help determine if SWCNTs are present and potentially identify the source. For example, when Ni was used as a catalyst in SWCNTs synthesis, the Ni:C ratio in the product was quite high, varying between 0.20 and 0.37 (Figure 1a). These proportions are orders of magnitude higher than those of other common aerosol sources, such as coal $\left(\mathrm{Ni}: \mathrm{C}=2.2 \times 10^{-5}\right)$, petroleum $(\mathrm{Ni}: \mathrm{C}=7.7 \mathrm{x}$ $\left.10^{-5}\right)$, and continental crust $\left(\mathrm{Ni}: \mathrm{C}=5.9 \times 10^{-3}\right)$ [26]. If crustal-derived dust occurred in an air sample at $1 \mathrm{ug} \mathrm{m}^{-3}$ and has $1 \%$ carbon content, then the crustal Ni:C ratio implies 0.06

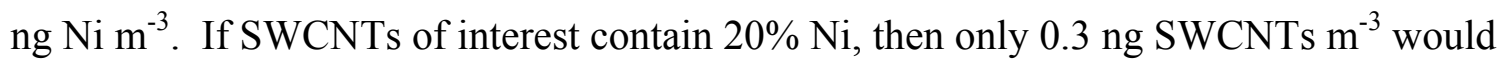
be needed to double the Ni in the air. Therefore, $\mathrm{ng} \mathrm{m}^{-3}$ levels of SWCNTs would result in Ni levels six times higher than background. Thus, Ni measures can detect industrially produced SWCNTs at $\mathrm{ng} \mathrm{m}^{-3}$ levels in air samples. Moreover, key metal ratios might be diagnostic. The Ni:Y of the commercial SWCNTs varied between 2.9 and 4.9 (Figure 1a), and these ratios are also distinct from coal $(\mathrm{Ni}: \mathrm{Y}=1.3)$, petroleum $(\mathrm{Ni}: \mathrm{Y}=66,000)$, and continental crust $(\mathrm{Ni}: \mathrm{Y}=0.9)[26]$. Hence, the proposed natural sources of SWCNTs (e.g., products of combustion processes [27]) could be distinguished from some industrial sources based on metal content. This will be particularly useful for identifying responsible parties if SWCNT manufacturers are called on to remediate SWCNTs found in the environment, as other users of industrially important materials have in the past (e.g., DuPont ${ }^{\mathrm{TM}}$ for perfluorooctanoic acid (PFOA) \$108 Million for Parkersburg, WV [28]; General Electric ${ }^{\mathrm{TM}}$ for polychlorinated biphenyl ethers (PCBs), \$115 Million for Hudson River, NY [29]; and W.R. Grace ${ }^{\mathrm{TM}}$ for asbestos, \$58 Million for Libby, MT [30]). 


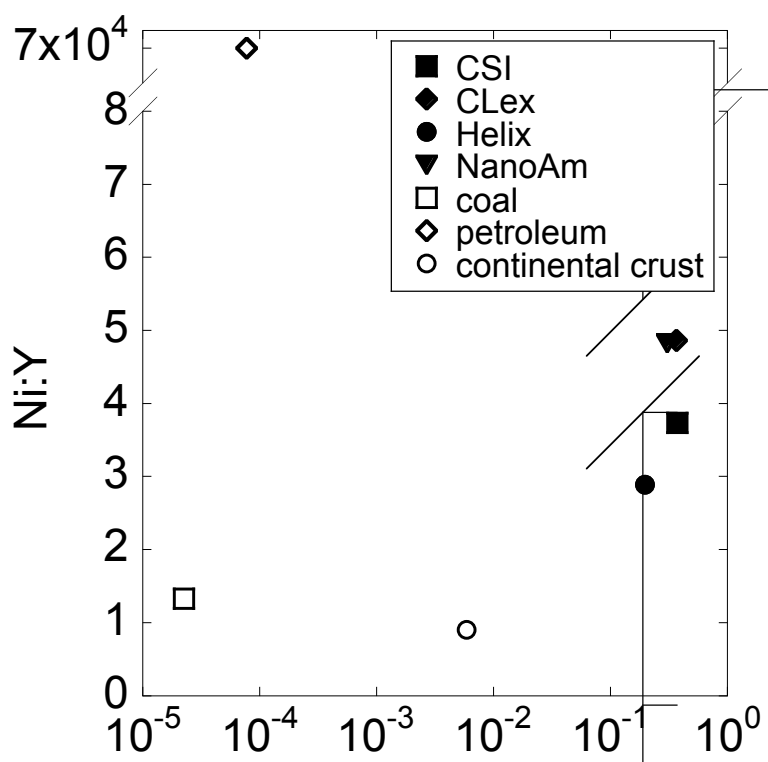

(a)

$\mathrm{Ni}: \mathrm{C}$

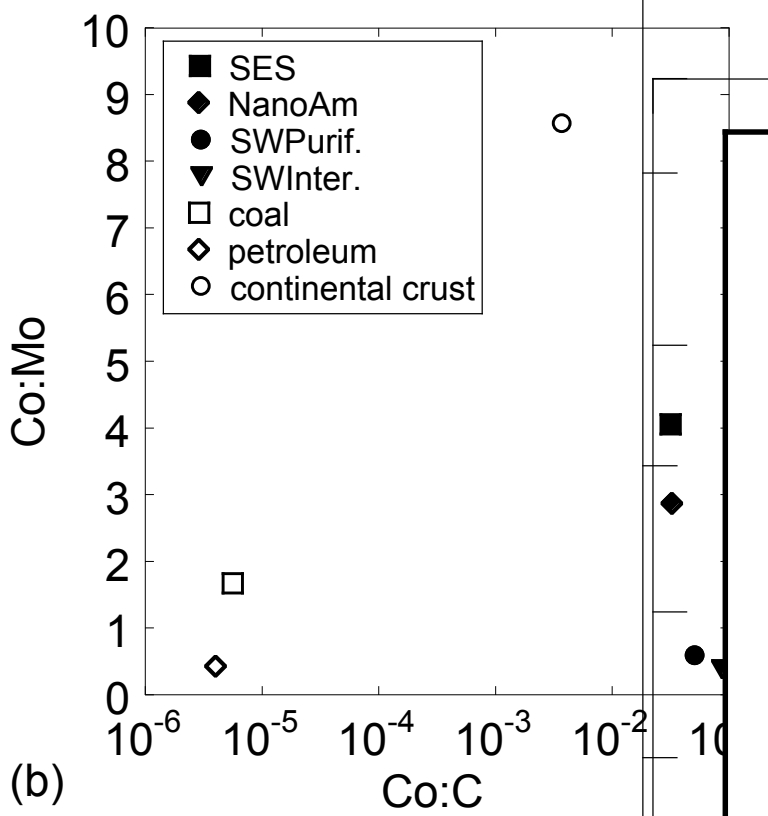

Figure 1. Metal ratios in SWCNTs and other aerosol sources. (a) Ni:Y ratios in SWCNTs are distinct from those in coal, petroleum, and continental crust. Furthermore, the metal abundance of these catalysts relative to the $\mathrm{C}$ content is much higher than $\mathrm{Ni}$ abundance in other materials. These distinct metal contents could be useful tracers of SWCNTs in environmental samples. (b) Co:Mo ratios in SWCNTs vary (see text) and overlap with some natural sources. However, the relative abundance of $\mathrm{Co}: \mathrm{C}$ is distinct from coal, petroleum, and continental crust and could be used to identify SWCNTs in aerosol concentrates. The relative metal and carbon contents of coal, petroleum, and continental crust were calculated from data in Klee and Graedel [26]. 
Currently, there is a narrow range of Ni:Y ratios used in commercial SWCNT production. There is more variability in the relative amounts of Co:Mo that successfully yield SWCNTs. When only Co and Mo are used as metal catalysts in SWCNT synthesis, the Co:Mo ratio was $0.31 \pm 0.01$ or $0.59 \pm 0.04$ (for SWIntermediate and SWPurified, respectively), whereas the ratio is much higher $(2.9 \pm 0.1$ for NanoAm and $4.0 \pm 0.2$ for $\mathrm{SES})$ for SWCNTs grown from $\mathrm{Fe} / \mathrm{Co} / \mathrm{Mo}$ catalyst mixtures. Thus, metal catalyst ratios may be used to distinguish SWCNTs produced by different SWCNT manufacturers.

Unlike Ni:Y abundances, the Co:Mo ratios in industrially synthesized SWCNTs $(0.31$ \pm 0.01 to $4.0 \pm 0.2$ ) overlap with those of coal (1.67) and petroleum (0.43), making it difficult to identify natural and anthropogenic SWCNT sources using metal ratios alone. However, SWCNTs have relatively high Co:C content ( 0.31 and 0.88 ) compared to coal $\left(\mathrm{Co}: \mathrm{C}=5.6 \times 10^{-6}\right)$, petroleum $\left(\mathrm{Co}: \mathrm{C}=4.0 \times 10^{-6}\right)$, and continental crust $(\mathrm{Co}: \mathrm{C}=3.7 \times$ $\left.10^{-3}\right)$ [26]. If crustal-derived dust occurred in an air sample at $1 \mathrm{ug} \mathrm{m}^{-3}$ and has $1 \%$ carbon content, then the crustal Co:C ratio implies $0.04 \mathrm{ng} \mathrm{Co} \mathrm{m}^{-3}$. If SWCNTs of interest contain $3 \% \mathrm{Co}$, then only $1.3 \mathrm{ng}$ SWCNTs ${ }^{-3}$ would be needed to double the Co in the air. Therefore, $4 \mathrm{ng} \mathrm{m}^{-3}$ levels of SWCNTs would result in Co levels six times higher than background. This sensitivity for Co-containing SWCNTs is slightly less than that for Ni-containing SWCNTs and results from the higher Co content of continental crust and lower Co content of SWCNTs, as compared to the Ni content of the respective materials. Nevertheless, Co measures can detect industrially produced SWCNTs at several $\mathrm{ng} \mathrm{m}^{-3}$ levels in air samples. Thus, the unique metal-to-carbon content of SWCNTs can be useful for tracing these emerging contaminants as they are released to and travel through the environment.

Total carbon and nitrogen. The total carbon content of the SWCNTs ranged from $60-97 \%$ (Table 4). The measured values agree with the manufacturer-reported carbon contents, except for SWPurified and Nanocs. SWPurified contained $81 \pm 2 \% \mathrm{C}$ when measured by elemental analysis, whereas $93 \% \mathrm{C}$ was reported by the manufacturer. This offset may result from differences in analytical methods, as SWeNT Inc. determines the elemental content using $\mathrm{x}$-ray photoelectron spectroscopy (XPS). XPS is used to determine the chemical composition of a surface (typically the upper $10 \mathrm{~nm}$ or less). It is possible that thick $(>10 \mathrm{~nm})$ amorphous carbon coatings in the SWPurified sample biased 
the carbon content to an artificially high value. Nanocs contained $66.3 \pm 0.8 \% \mathrm{C}$ even though this product was listed as "high purity." The Nanocs C content is quite low for traditionally purified SWCNTs; and when contacted, Nanocs, Inc. explained that they emphasize structural purity, eliminating amorphous carbon and retaining intact SWCNTs without removing metal catalysts. Metal analyses confirm that this sample was not purified of metal catalysts. Purified and unpurified SWCNTs are often difficult to distinguish without chemical analyses, and such subtleties in nomenclature may confound toxicity, environmental transport, and SWCNT application studies.

An intermediate product in SWCNT synthesis, SWIntermediate, had an unusually low $\mathrm{C}$ content $(5.7 \pm 0.2 \%)$, primarily due to the presence of inorganic growth substrate $\left(\mathrm{SiO}_{2}\right)$. Correcting for the presence of the substrate gave a calculated $\mathrm{C}$ content of $76 \pm$ $2 \%$. While this corrected result certainly puts the product in the range seen for other SWCNTS, it is clear that the carbon contents of these ostensibly carbonaceous materials were quite variable.

Similarly, there was a broad range in the SWCNT-C and amorphous C content of these materials, irrespective of the reported purity level (Table 4). Arc-produced samples contained between $23 \pm 1$ and $44 \pm 1 \%$ SWCNT-C, whereas CVD-generated SWCNTs contained between $41 \pm 1$ and $81 \pm 1 \%$ SWCNT-C. Again, the SWIntermed. sample contains $82 \pm 3 \% \mathrm{SiO}_{2}$ growth substrate (Table 2), and correcting for the presence of this material gave $71 \pm 3 \%$ SWCNT-C. Perhaps surprisingly, some purified samples, NanoAm and SWP, contained "amorphous C" at or above the SWCNT-C content. We suspect that this was due to the formation of a partially oxidized carbon phase during the SWCNT purification process, which typically involves thermal and/or chemical oxidation steps. Clearly, these lower-thermal-stability (relative to SWCNT-C) phases were not removed during subsequent purification steps. 


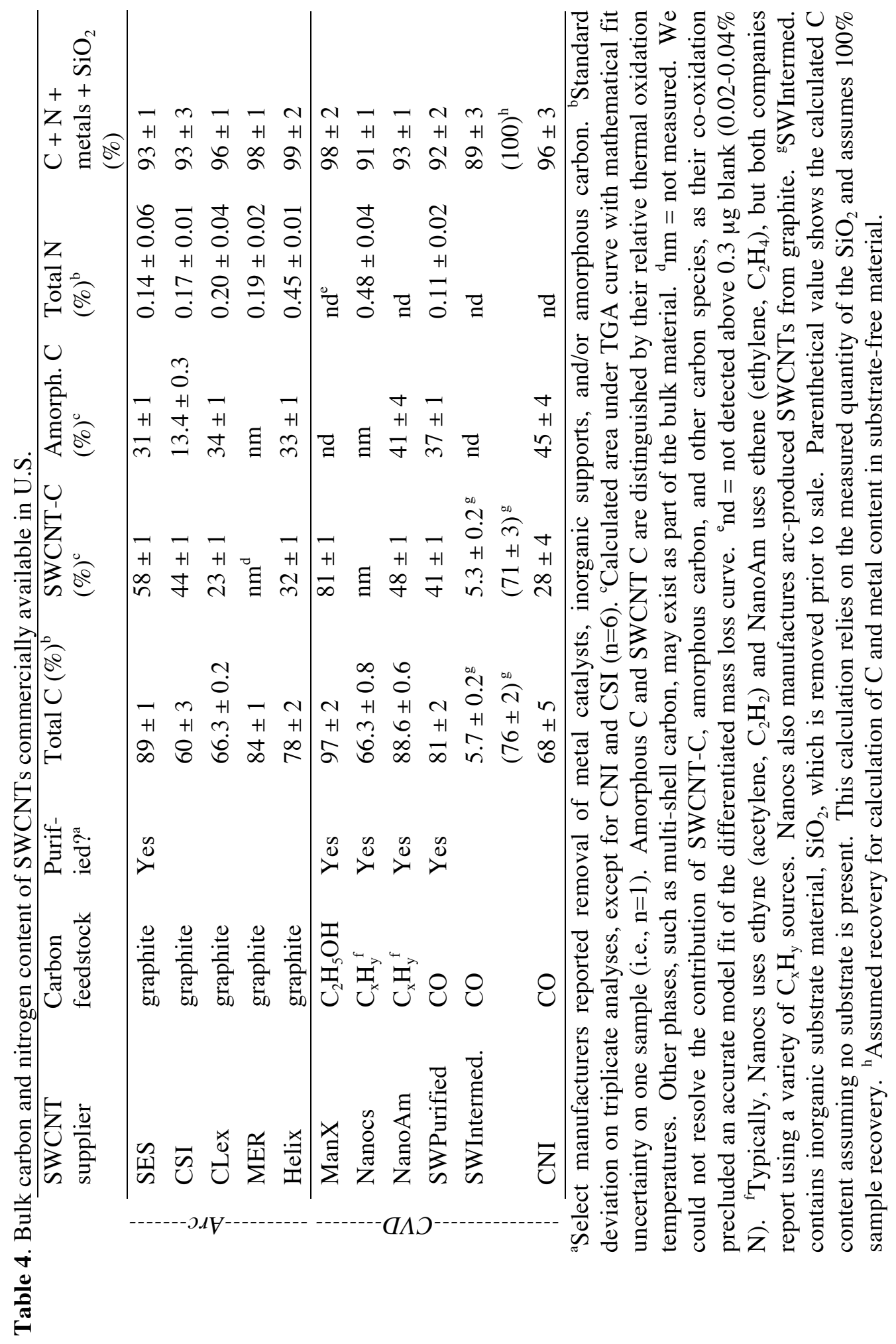


Given the diversity of procedures and metals used in SWCNT synthesis, and the influence of those choices on the thermal stability and relative abundance of SWCNTs $[31,32]$, it is difficult to compare directly our results with the literature. Nevertheless, we note that CarboLex samples analyzed by the same TGA method exhibited roughly $25 \%$ amorphous C and 30\% SWCNT-C (by inspection of the mass-loss thermogram) [31], in agreement with our results. In addition, Itkis et al. [33] report 31.8\% SWCNT purity for the CSI sample, analyzed by near-infrared spectroscopy. Our TGA results indicate that this sample contained $44 \pm 1 \%$ SWCNT-C. This discrepancy may reflect the differing analytical biases of the two methods. For example, the thermogram in this sample indicated the presence of purported multi-shell carbon [31] co-oxidizing with the SWCNTs. This co-oxidation could result in a mathematical overestimation (due to curve fitting procedure) of the SWCNT content. Alternatively, the discrepancy could result from differences in the commercially available product over the time frame of the two studies.

Unfunctionalized SWCNTs are expected to have very low N contents. However, we found that all arc-synthesized SWCNTs contained measurable amounts of $\mathrm{N}$, ranging from 0.14 to $0.45 \%$ (Table 4 ). In contrast, catalytic chemical vapor deposition (CVD)produced materials did not have detectable quantities of $\mathrm{N}(0.02-0.04 \% \mathrm{~N})$, except for SWPurified and Nanocs. We hypothesize that the presence of $\mathrm{N}$ in SWPurified results from $\mathrm{N}$ incorporation during (1) SWCNT synthesis, (2) the purification process, especially if $\mathrm{HNO}_{3}$ is used, or (3) post-manufacture processing and transport. Additionally, although Nanocs, Inc. currently employs CVD as their principle fabrication method, they also use arc-discharge synthesis. It is possible that this particular batch of SWCNTs was arc produced. The high-energy environment of arc-discharge synthesis may favor the incorporation of $\mathrm{N}$, even if derived from atmospheric $\mathrm{N}_{2}$ [34], into SWCNTs. The direct incorporation of $\mathrm{N}$ into the carbon-based skeleton can influence the helicity and, therefore, the conductivity of the nanotube [35]. Thus, manufacturers designing synthetic processes for a particular application may choose low- or high-energy fabrication depending on the desired tube chirality. If the $\mathrm{N}$ is incorporated into the nanotube itself, the added functionality may alter SWCNT persistence in the environment by influencing the surface charging and increasing susceptibility to transformations. 
Moreover, if the $\mathrm{N}$ occurs as part of the population of aromatic hydrocarbon sideproducts (which will be discussed in more detail below), there is cause for concern regarding the toxicities derived from $\mathrm{N}$-heterocyclic compounds [36].

From a mass balance perspective, the measured quantities of $\mathrm{C}, \mathrm{N}$, metals, and $\mathrm{SiO}_{2}$ accounted for between $89 \pm 3$ and $99 \pm 2 \%$ of the SWCNT materials (Table 4). None of our analyses quantified the oxygen content of the SWCNTs, and so the remaining fraction of unaccounted material could contain oxygen. Such oxygen could be present as functional groups on the SWCNTs or, perhaps more likely, as metal oxides. If we assume that metal oxides are present at $50-100 \%$ of the SWCNT-associated metal content and in representative stoichiometries (i.e., $\mathrm{NiO}, \mathrm{Y}_{2} \mathrm{O}_{3}, \mathrm{Fe}_{2} \mathrm{O}_{3}, \mathrm{MoO}_{3}$, and $\mathrm{CoO}$ (or, equivalently, $\left.\mathrm{CoMoO}_{4}\right)$ ), we find that oxygen can account for the missing mass in all of the AP-SWCNTs (Figure 2). In the case where we assume 100\% of the metal is present as metal oxide, the total mass balance is overestimated by as much as $2.5 \%$ (outside of the analytical error), whereas the mass balance is within the analytical error when we assume $50 \%$ of the metal is present as metal oxide. (For the intermediate product (SWIntermed.), no amount of metal oxide can account for the missing material, suggesting that we have failed to quantify some portion of the material. TGA results confirm that we are not accounting for approximately $10 \%$ of the AP-SWCNT product). In purified SWCNTs, metal oxides cannot account for the missing mass, and 2-6\% of the material remains unexplained. We propose that purified materials contain oxygen functional groups within the SWCNT lattice, as oxidizing acids used during purification can induce such functionality, especially at SWCNT ends and defects. These oxygen functional groups, along with acid residues (e.g., $\mathrm{HCl}, \mathrm{HNO}_{3}$, and $\mathrm{H}_{2} \mathrm{SO}_{3}$ ), could account for the unquantified proportion of the purified SWCNTs. Whether present as metal oxides or functional groups on the SWCNTs, oxygen functionalities contributing up to $10 \%$ by weight of the SWCNTs would dramatically affect the surface properties of these nanoparticles (e.g., conferring $\mathrm{pH}$-dependent charging in aqueous suspensions), which will be important for many applications that rely on SWCNT adsorptivity (e.g., water filtration) and suspension stability. 


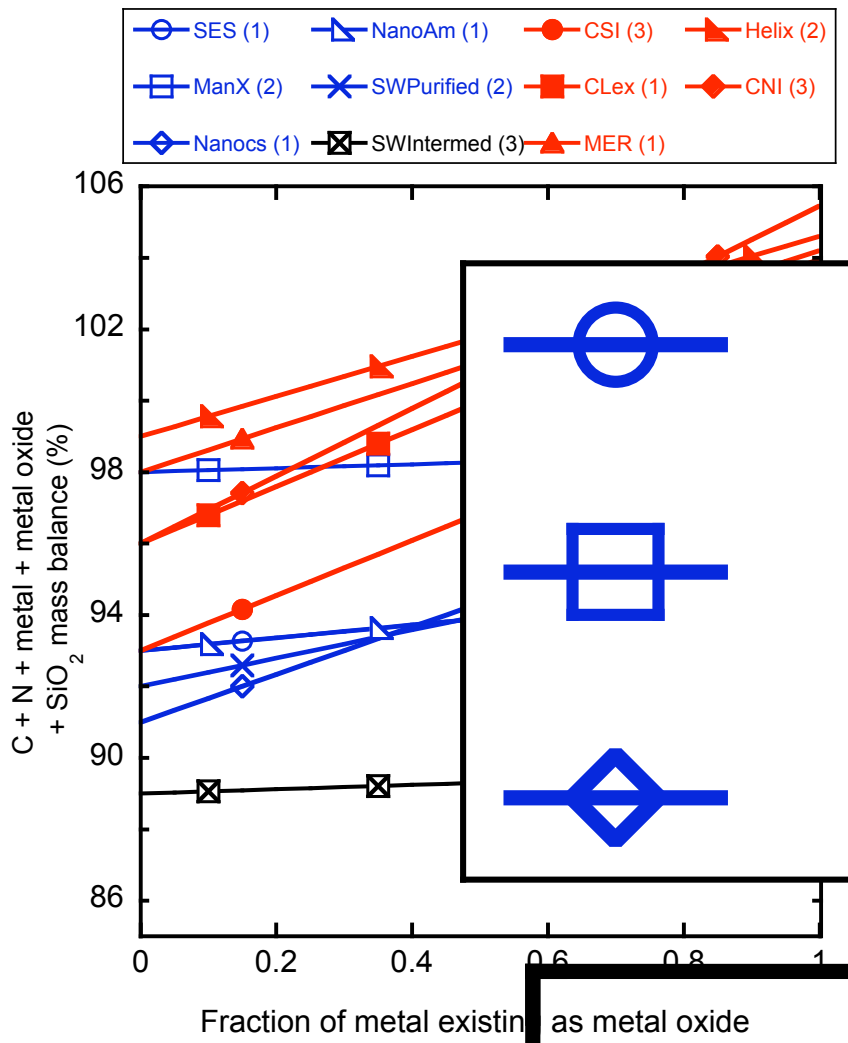

Figure 2. Total accounted SWCNT mass as a function of assumed metal oxide content. For this calculation, we assumed that metal oxides existed in representative stoichiometries (i.e., $\mathrm{NiO}, \mathrm{Y}_{2} \mathrm{O}_{3}, \mathrm{Fe}_{2} \mathrm{O}_{3}, \mathrm{MoO}_{3}$, and $\mathrm{CoO}$ (or, equivalently, $\mathrm{CoMoO}_{4}$ )). Using the quantified metal abundances, we calculated the mass of oxygen that would be present as metal oxide. The uncertainty in the calculation is given in parentheses in the legend, next to the sample name. (Error bars are omitted for figure clarity). For APSWCNTs (filled, red symbols), metal oxides easily account for the unidentified material if we assume that $50 \%$ of the SWCNT-associated metal is present as metal oxide. For purified materials, (open, blue symbols) oxygen present as metal oxides cannot account for the total mass of the SWCNT, and oxygen present in the SWCNT carbon lattice could explain the missing mass fraction. For the intermediate SWCNT product (black, hatched symbols), SWInermed., no amount of metal oxide can explain the unaccounted fraction of material. 
Stable carbon isotope composition of SWCNTs. SWCNTs exhibited a wide range in $\delta^{13} \mathrm{C}_{\mathrm{VPDB}}$ values (-53.2 to $-23.5^{\circ}$ oo, Figure 3, see Experimental Methods for definition of $\delta{ }^{13} \mathrm{C}$ notation). The observed ${ }^{13} \mathrm{C}$ abundances can result from either the $\mathrm{C}$ source material or isotopic fractionation during fabrication and post-production treatments. Carbon isotopic fractionation typically diminishes with increasing reaction temperature [37], and we expect that such effects are minor at SWCNT synthesis temperatures, which often exceed $700^{\circ} \mathrm{C}$. Thus, we believe that the diverse ${ }^{13} \mathrm{C}$ abundances primarily reflect the varied ${ }^{13} \mathrm{C}$ content of their respective source materials (Table 4). A variety of carbon source materials are used for CVD SWCNTs, including ethanol, carbon monoxide, and ethane [5]. Ethanol can be produced from the sugar derived from $\mathrm{C}_{3}$ plants (woody plants, $\delta{ }^{13} \mathrm{C}_{\mathrm{VPDB}}=-30$ to $-25 \%$ or or $\mathrm{C}_{4}$ plants (maize, grasses, $\delta{ }^{13} \mathrm{C}_{\mathrm{VPDB}}=-20$ to $-14 \%$ ) [38]. Carbon monoxide is typically produced from methane, which can be depleted in ${ }^{13} \mathrm{C}\left(\delta{ }^{13} \mathrm{C}_{\mathrm{VPDB}}\right.$, biogenic methane $=-110$ to $-50 \%$ \% [39]. Graphite (a carbon source for arc SWCNTs), ethene, and ethyne are all industrially produced from petroleum coke. Petroleum cokes and associated compounds can have a range of ${ }^{13} \mathrm{C}$ contents depending on the isotopic signature of the source reservoir and can range from -34 to $-22 \%$ o [40-42]. 


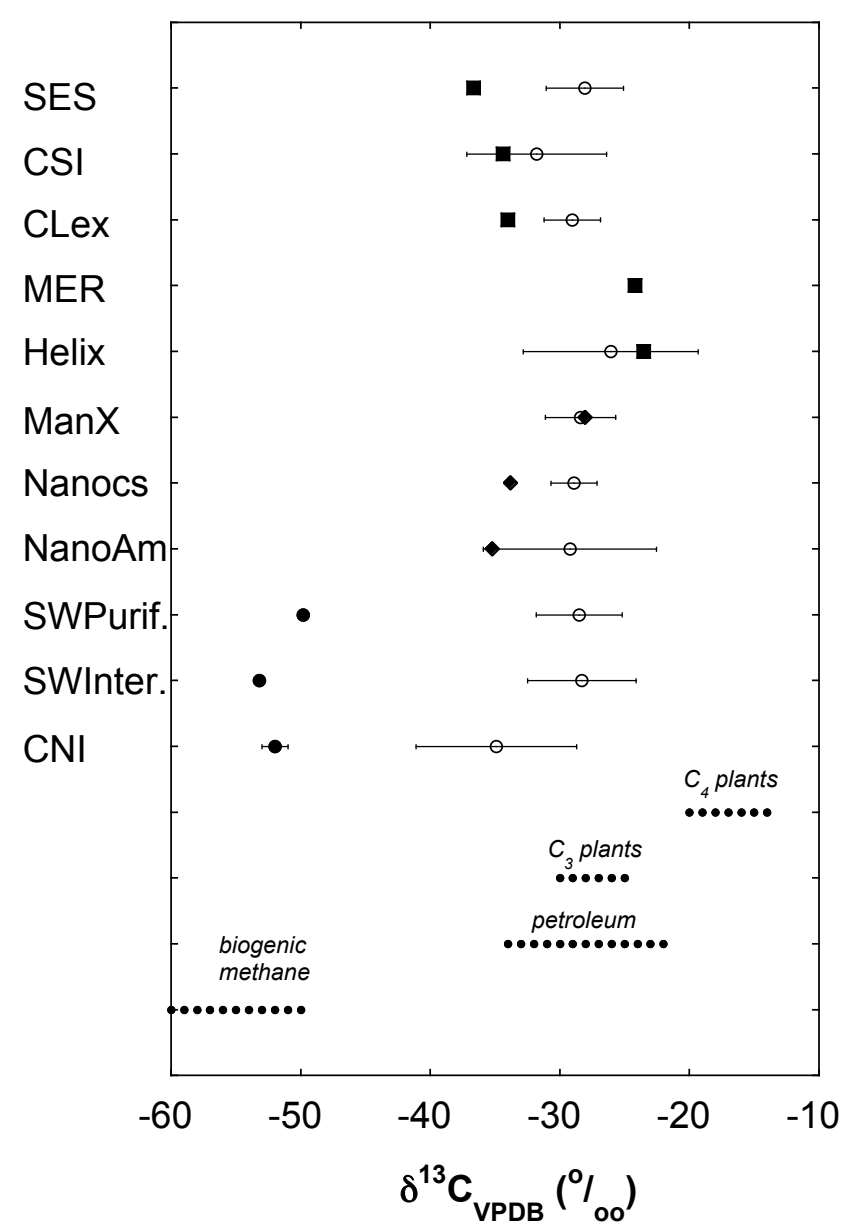

Figure 3. Stable carbon isotopic signatures of SWCNTs, SWCNT-associated solvent extractable material, and selected carbon sources. SWCNTs synthesized from arcdischarge ( $\boldsymbol{\square})$, CVD $(\bullet)$, and CO-CVD (๑) exhibit a broad range of ${ }^{13} \mathrm{C} /{ }^{12} \mathrm{C}$ ratios, primarily resulting from the distinct isotopic signatures of their respective starting materials (OO). Graphite is industrially produced from petroleum coke $\left(\delta^{13} \mathrm{C}_{\mathrm{VPDB}}\right.$, petroleum-derived $=-34$ to $-22 \%$ ) [40-42], and provides a carbon source for arc-discharge synthesized SWCNTs. A variety of carbon source materials are used for CVDsynthesized SWCNTs, including ethanol and carbon monoxide. Ethanol can be produced from the sugar derived from $C_{3}$ plants (woody plants, $\delta^{13} C_{V P D B}=-30$ to $-25 \%$ ) or $C_{4}$ plants (maize, grasses, $\delta^{13} \mathrm{C}_{\mathrm{VPDB}}=-20$ to $-14^{\mathrm{o}} \%$ ) [38]. Carbon monoxide is typically produced from methane $\left(\delta^{13} \mathrm{C}_{\mathrm{VPDB}}\right.$, biogenic methane $\left.=-110--50 \%\right)[39,42]$. Error bars represent one standard deviation on quadruple analyses, except SES, MER, SWIntermed., Helix $(n=2)$, and CLex $(n=3)$. The ${ }^{13} \mathrm{C}$ content of dichloromethane solvent extractable material (O) is consistent with petroleum-derived sources, characteristic of ambient hydrocarbons. Error bars represent one standard deviation on triplicate measurements. The larger relative error of the extracts (as compared to the SWCNTs) is due to the small quantity of extracted material. 
Arc SWCNTs have $\mathrm{d}^{13} \mathrm{C}_{\mathrm{VPDB}}$ values ranging from -36.7 to $-23.5 \%$, indicative of graphitic sources. The ${ }^{13} \mathrm{C}$ abundance of $\operatorname{ManX}(-28.0 \%$ oo $)$ is consistent with growth from a $\mathrm{C}_{3}$-derived ethanol [38]. Ethene-derived NanoAm $(-35.2 \%$ oo $)$ had a ${ }^{13} \mathrm{C}$ content similar to Nanocs $(-33.8 \%$ oo , which has a proprietary $\mathrm{C}$ source. Given the broad and overlapping range of ${ }^{13} \mathrm{C}$ abundances, it is not possible to differentiate arc and CVD SWCNTs using $\mathrm{C}$ isotopic techniques. Conversely, CO-CVD SWCNTs have a distinct ${ }^{13} \mathrm{C}$ composition (51.7 to $-49.8 \%$ oo, reflecting growth from a ${ }^{13} \mathrm{C}$-depleted feedstock. This unique isotopic signature may be another useful tracer of CO-SWCNTs and their metabolites in both laboratory and field studies. For example, the ${ }^{13} \mathrm{C}$ abundance of CO-SWCNT transformation products will be depleted relative to the bulk carbon of most systems (i.e., SWCNT-derived biomolecules will have a different isotopic signature than biomolecules from other carbon sources). In addition, CO-derived SWCNTs' isotope signature is distinct from the bulk isotopic content of marine seawater and sedimentary organic matter $(-30$ to $-20 \%$ o [38]. This isotopic fingerprint may be particularly valuable for the development of analytical methods to quantify SWCNTs in complex environmental matrices, as the isotopic content of an artificial sample (e.g., a lab-generated aerosol sample containing CO-derived SWCNTs) could be used independently to calculate the quantity of nanotubes persisting through each step of the analysis (i.e., sample collection, separation, and quantification). A similar approach would allow one to quantify SWCNTs in mixture-based applications, such as polymer composites and pharmaceutical vectors.

Total solvent-extractable material (TSEM). Amorphous carbon and graphitic nanoparticles are considered the principle components of C-based SWCNT impurities [12], accounting for up to $30 \%$ of as produced-SWCNT material. In addition, amorphous carbon formation plays an important role in catalyst deactivation during CVD growth, resulting in sub-optimal carbon nanotube formation [43], and influences the electronic character of the SWCNT [44]. The chemical composition of this amorphous carbon fraction is largely uncharacterized, and one of our objectives was to develop a more detailed description of the SWCNT co-products, their behavior, and their origin through solvent extraction. 
TSEM isotopic composition and hydrocarbon origin. The ${ }^{13} \mathrm{C}$ abundances of the DCM solvent extracts of all the SWCNTs were approximately $-29 \%$ oo (range $-35 \pm 6$ to $-26 \pm 7 \%$, Figure 3 ), irrespective of the ${ }^{13} \mathrm{C}$ content of the SWCNTs. This suggests most of the TSEM derived from a common source for all of the SWCNTs. The ${ }^{13} \mathrm{C}$ signature of the TSEM was consistent with that of ambient petroleum-derived compounds $(-34$ to $-22 \%$ o $)[40,41]$, and SWCNTs could be acquiring hydrocarbons through contact with industrial apparatus and packaging material, as well as through exposure to ambient vapors and aerosols. The presence of phthalates (ubiquitous organic compounds used in the manufacture of plastics) in all of the solvent extracts, as compared to our phthalate-free laboratory blanks, also supported the contention that SWCNTs have a tendency to sorb organic contaminants from their surroundings. Sorbed organic material influences the electronic properties of SWCNTs [19], and thus manufacturers concerned with the electronic integrity of their nanotubes should take care to minimize SWCNT contact with plastics, other organic-rich materials, and urban air. These data indicate that SWCNTs released to the environment may continue to sorb organic compounds, influencing the mobility and persistence of both natural and pollutant compounds. Depending on the strength of the sorptive interactions, SWCNTs will either act as a local sink for anthropogenic contaminants or as a vector for distributing them in the environment.

TSEM mass and metal reactivity. The quantity of solvent-extractable material was remarkably low ( $<1 \%$ in dichloromethane (DCM), $<5 \%$ in toluene, Table 5 ), suggesting that carbonaceous impurities were either (1) not soluble in DCM or toluene due to their macromolecular/particulate nature, (2) strongly sorbed to SWCNTs, or (3) degraded during the solvent extraction procedure. While we would not expect amorphous or graphitic nanoparticles to be soluble, it is somewhat surprising that small ( $<350 \mathrm{amu}$ ), soluble molecules contribute less than 5\% of the SWCNT co-products [45, 46]. If such molecules are formed, preserved, and associated with the SWCNTs, we would expect that milliliter quantities of apolar solvent would efficiently extract them from milligram quantities of SWCNTs, based on our physical-chemical understanding of hydrocarbon sorption [47]. Thus, we hypothesized that a degradation reaction was consuming some of the low molecular weight compounds during extractions. As noted 
previously, both purified and unpurified SWCNTs contain substantial quantities of metals (Table 3). Reduced metals are able to react with dichloromethane to form chloromethyl radicals, and such radicals can react with expected components of the TSEM, such as PAHs [48]. These transformations would result in losses of PAHs in halogenated solvent extractions.

To test this hypothesis, we compared the recovery of three added PAH internal standards ( $d_{10}$-acenaphthene, $m$-terphenyl, $d_{12}$-perylene) with toluene versus DCM extractions of the SWCNTs. DCM extractions of SWCNTs showed poor recoveries for all three internal standards (Figure 4). In contrast, the toluene extractions were very efficient $\left(96 \pm 8 \%\right.$ to $114 \pm 8 \%$ ) for $d_{10}$-acenaphthene and $m$-terphenyl from all SWCNTs, and the $d_{12}$-perylene was also recovered better by the toluene. Interestingly, DCM extractions of purified SWCNTs showed markedly worse recoveries of $m$-terphenyl and $d_{12}$-perylene than DCM extractions of unpurified SWCNTs. Purification processes typically remove the amorphous carbon coating of the SWCNT-associated metal catalysts [12], and while decreasing the overall metal abundance, purification could increase the effective surface area of available metal catalyst. The poor internal standard recoveries in purified SWCNT-DCM extracts are consistent with degradation of PAHs by radicals generated via metal-halogenated solvent reaction. These data suggested that metals are not "inaccessible to the outside environment," [2] as some have suggested, but rather, the metals are available to participate in reactions.

Given the difficulty of removing metal catalysts from SWCNTs with 100\% efficiency (Table 3) and the apparent increase in metal reactivity with SWCNT purification (Figure 4), manufacturers and users must consider the effect of metal catalysts in their products. For example, the metal content of SWCNTs designed to deliver pharmaceuticals to cell targets [49] will influence the lifetime of the drug in the body. Additionally, the presence of reactive metals will almost certainly influence the toxicity of the nanomaterial $[10,20$, 21]. SWCNTs released to the environment could also react with halogenated contaminants, generating radical species and degradation products with elevated toxicity $[50,51]$. For example, zero-valent metals are used to remediate chlorinated ethenes via reductive dehalogenation to produce toxic vinyl chloride [50]. Furthermore, such reactions result in oxidation of the metal catalyst, which will alter the solubility of the 
metal, the SWCNT's ability to carry and dissipate charge [18], and the charging of the nanoparticle surface. Consequently, risk assessment models must consider the presence of the reactive metal, and we cannot continue to ignore the presence of catalytic impurities in manufactured nanomaterials.

Table 5. Mass of solvent-extractable material from SWCNTs

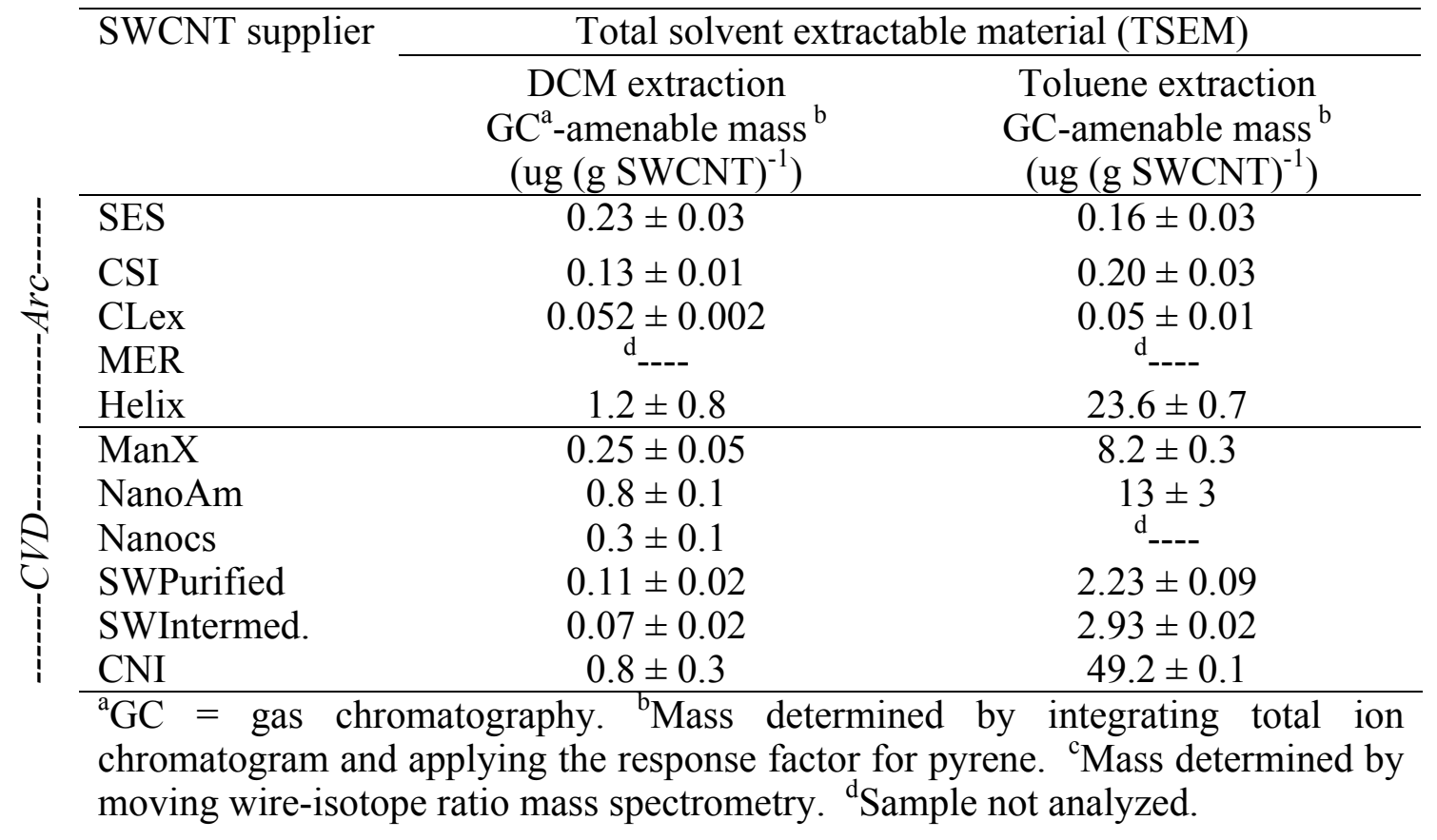




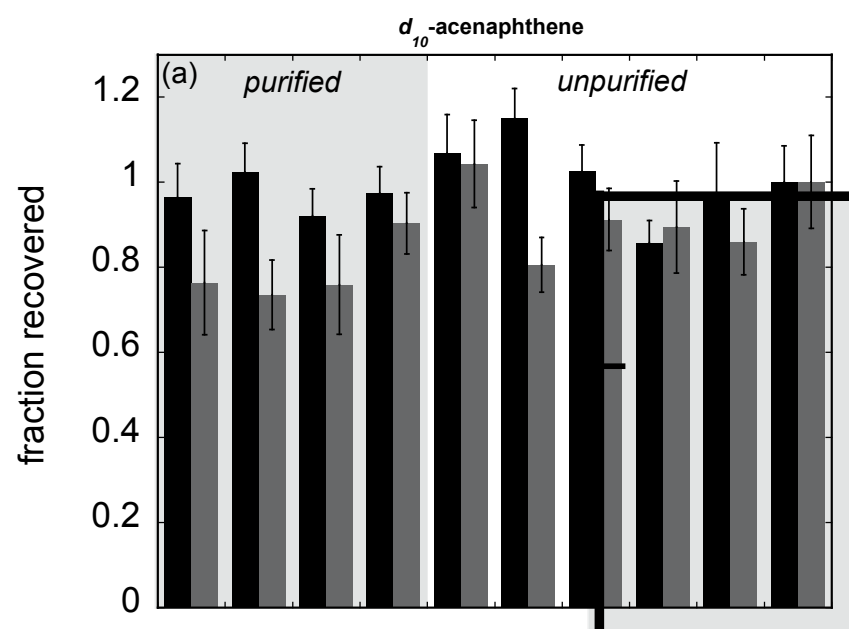

Figure 4. Polycyclic aromatic hydrocarbon internal standard recoveries. Recoveries of three PAH internal standards, $d_{10^{-}}$ acenapthene (a), $m$-terphenyl (b), and $d_{12}$-perylene (c), varied significantly between samples. Additionally, dichloromethane (dark gray) extractions of SWCNTs gave poorer recoveries than analogous toluene (black) extractions, especially in the case of $m$-terphenyl and $d_{12^{-}}$ perylene. These two compounds exhibit higher recoveries from unpurified (white background) samples than purified (light gray background) samples. Note the different scale necessitated by the $d_{12}$-perylene data. Here, the "no SWCNTs" recovery is off scale (fraction recovered $=1$ ). Recoveries are reported relative to the SWCNT-free controls ("no SWCNTs"), and error bars represent one standard deviation on duplicate analyses.

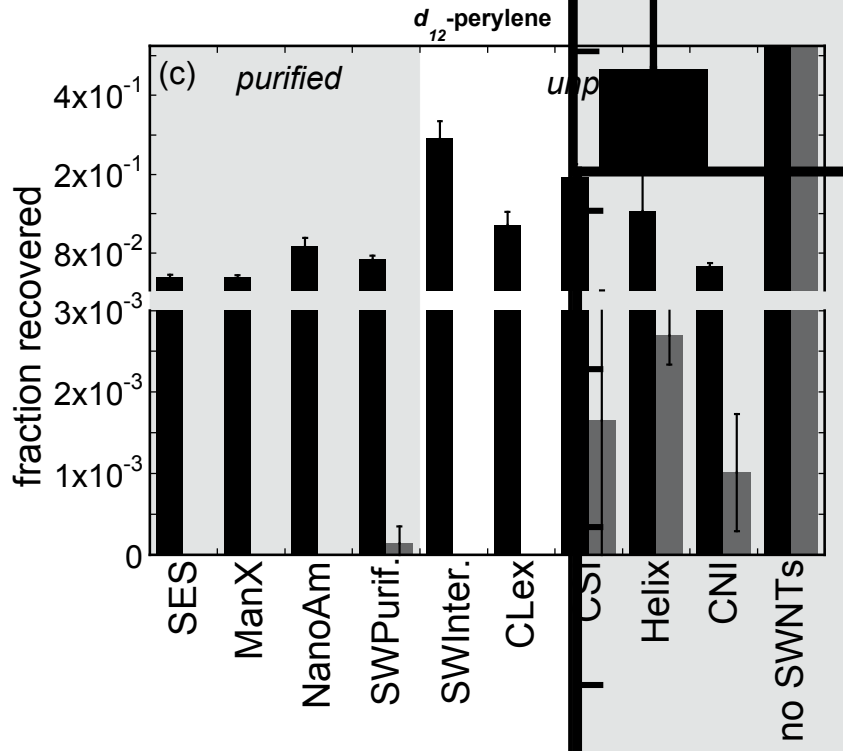


Solvent-extractable polycyclic aromatic hydrocarbons. SWCNTs are formed at elevated temperatures from reactive carbon species. PAHs share this formation mechanism, and we hypothesized that PAHs would be co-formed during SWCNT synthesis [49]. Based on the effectiveness of our toluene extractions for the lowmolecular-weight (LMW)-PAHs ( $<200 \mathrm{amu}$ ), we determined the abundance and identity of such PAHs for the different SWCNT samples (Table 6). All samples contained naphthalene $\left(0.035 \pm 0.002\right.$ to $\left.112 \pm 4 \mathrm{ug}(\mathrm{mg} \mathrm{SWCNT})^{-1}\right)$, and some carried additional PAHs (CSI, Helix, ManX, Nanocs, SWPurified, NanoAm). CLex and CNI contained a full suite of PAHs, including benzo[b]fluoranthene $(0.07 \pm 0.03$ and $0.28 \pm 0.01 \mathrm{ug}$ (g SWCNT $)^{-1}$, respectively). Since the isotopic analyses indicated that the majority of SWCNT-associated hydrocarbons were derived from the environment rather than the SWCNT synthesis (Figure 4), the same may be true of the PAHs. Naphthalene and phenanthrene are present in many of the extracts, and they often appear in the greatest abundance. These compounds are common air contaminants, and we suspect that the PAH composition reported here is largely a function of the lifetime of the SWCNT sample (e.g., quality of air and carbonaceous materials to which it was exposed during manufacturer, transport, storage, and use). These results suggest that LMW-PAHs only contribute a small fraction of the amorphous material associated with SWCNTs. 


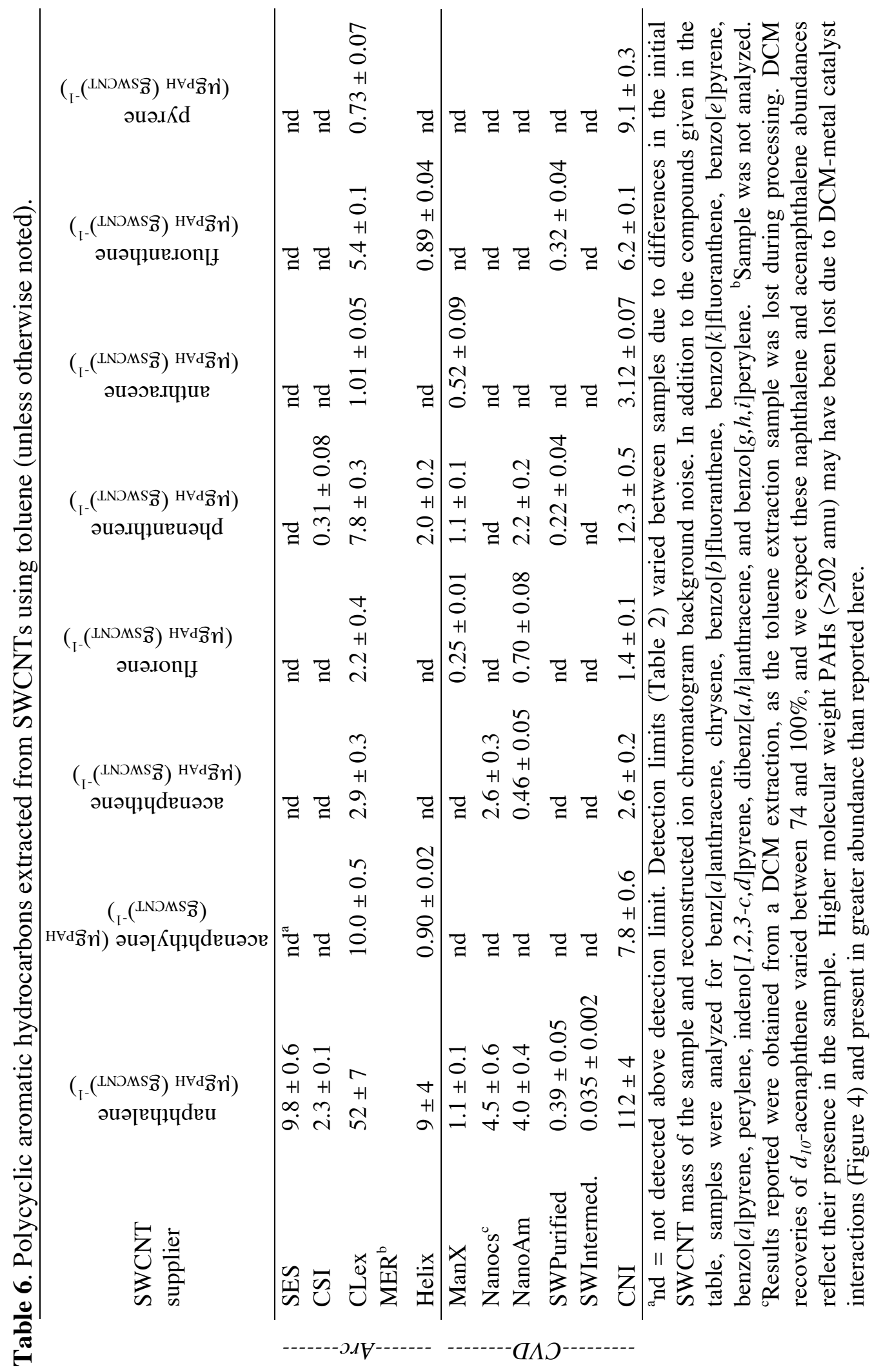




\section{IMPLICATIONS AND CONCLUSIONS}

With an industry-projected doubling rate of more than once per year [52], the anticipated growth of the carbon nanotube industry is nearly unprecedented, and SWCNTs will soon be in broad use by the general public in a variety of different applications. These materials will appear in the environment in the near future, and there is an urgent need to develop methods to study SWCNT transport [4]. These data provide metal-to-metal, metal-to-carbon, and carbon isotopic information that can be used, independently or in concert, as forensic tools to identify SWCNTs in natural and artificial samples.

In addition, we have suggested that SWCNTs, associated metals, and solvents can react to degrade aromatic hydrocarbons. This reactivity may be useful for chemical transformations. However, unintended reactions will affect the surface charge and conductivity of the nanomaterial, and thus the SWCNTs' suitability for a particular application.

Commercially available SWCNTs are highly diverse materials whose character and constituents vary with the production scheme and post-fabrication treatment. During these early stages of SWCNT research, investigators and regulators should be aware that there are significant differences in materials provided by different manufacturers, especially when cross-comparing and interpreting toxicological studies. Likewise, newly emerging nanomaterial risk assessments [53] must explicitly acknowledge these differences, as opposed to modeling the risk of a generic (i.e., over-simplified) SWCNT. Different SWCNTs will act as carriers of diverse metals and hydrocarbons to the environment. Furthermore, as both of these impurity classes affect the surface properties and reactivity of the SWCNTs, they will influence the overall environmental fate of the nanomaterials. Perhaps most importantly, SWCNT manufacturers can weigh the SWCNT-specific risk assessments and design synthetic processes to achieve environmental objectives while simultaneously considering performance and manufacturing cost. If we fail to use chemical understanding to strategically avoid unintended environmental consequences, the nanomaterial revolution may come to a halt with deteriorated public opinion, product bans, and expensive clean up efforts, such as those associated with other industrially important materials of the past (e.g., asbestos). 


\section{ACKNOWLEDGMENTS}

The authors are grateful for the assistance of D.X. Flores-Cervantes and J. MacFarlane (MIT), and A. Gagnon, C. Johnson, S. Manganini, D. Schneider, and S. Sylva (WHOI) with instrument operation and sample analysis. The authors thank J.

Kong (MIT), E. Kujawinski, B. Peucker-Ehrenbrink, and C. Miller (WHOI) for helpful discussion, as well as the SWCNT manufacturers for their cooperation and information regarding SWCNT synthesis. This work was supported by funds from the NSF (OCE 0223441), the NSF/SRC Engineering Research Center for Environmentally Benign Semiconductor Manufacturing, the Martin Family Society of Fellows for Sustainability, and the Earth System Initiative's Ignition Grant.

\section{REFERENCES}

[1] Wiesner, MR, Lowry, GV, Alvarez, P, Dionysiou, D and Biswas, P 2006 Environ. Sci. Technol. 40 (14) 4336-45

[2] Donaldson, K, Aitken, R, Tran, L, Stone, V, Duffin, R, Forrest, G and Alexander, A 2006 Toxicological Sciences 92 (1) 5-22

[3] Robichaud, CO, Tanzil, D, Weilenmann, U, Wiesner, MR 2005 Environ. Sci. Technol 39 (22) 8985-94

[4] Maynard, AD, Aitken, RJ, Butz, T, Colvin, V, Donaldson, K, Oberdörster, G, Philbert, MA, Ryan, J, Seaton, A, Stone, V, Tinkle, SS, Tran, L, Walker, NJ and Warheit, DB 2006 Nature 444 267-69.

[5] Liu, J, Shoushan, F and Dai, H 2004 MRS Bulletin April 244-50.

[6] Templeton, RC, Ferguson, PL, Washburn, KM, Scrivens, WA and Chandler, GT 2006 Environ. Sci. Technol. 40 (23) 7387-93

[7] Lam, C-W, James, JT, McCluskey, R and Hunter, R 2004 Toxicological Sciences 77 126-34

[8] Warheit, DB, Laurence, BR, Reed, KL, Roach, DH, Reynolds, GAM and Webb, TR 2004 Toxicological Sciences 77 117-25

[9] Hunter, KA and Liss, PS 1979 Nature 282 823-5 
[10] Nel, A, Xia, T, Mädler, L and Li, N 2006 Science 311 622-7

[11] Sayes, CM, Lian, F, Hudson, JL, Mendez, J, Guo, W, Beach, JM, Moore, VC, Doyle, CD, West, JL, Billups, WE, Ausman, KD and Colvin, VL 2006 Toxicology Lett. 161 135-42

[12] Haddon, R.C.; Sippel, J.; Rinzler, A.G.; Papdimitrakopoulos, F. Purification and separation of carbon nanotubes. MRS Bulletin. 2004, April, 252-259.

[13] Ding, RG, Lu, GQ, Yan, ZF and Wilson, MA 2001 J. Nanosci. \& Nanotechnol. 1 (1) 7-29

[14] Nicolaev, P, Bronikowski, MJ, Bradley, RK, Rohmund, F, Colbert, DT, Smith, KA and Smalley, RE 1999 Chem. Phys. Lett. 313 91-7

[15] Ingamells, CO 1970 Analytica Chimica Acta 52 (2) 323-34

[16] McNichol, AP, Osborne, EA, Gagnon, AR, Fry, B and Jones, GA 1994 Nuc. Instrum. Methods Phys. Res. B92 (1-4) 162-5

[17] Sessions, AL, Sylva, SP and Hayes, JM 2005 Anal. Chem. 77 6519-27

[18] Ruppalt, LB and Lyding, JW 2007 Nanotechnology 18215202 (DOI:10.1088/0957-4484/18/21/215202)

[19] Lam, C-W, James, JT, McCluskey, R, Arepalli, S and Hunter, RL 2006 Critical Reviews in Toxicology 36 189-217

[20] Liu, X, Gurel, V, Morris, D, Murray DW, Zhitkovich, A, Kane, AB and Hurt, RH 2007 Advanced Materials DOI:10.1002/adma.200602696

[21] Guo, L, Morris, DG, Liu, X, Vaslet, C, Hurt, RH and Kane, AB. 2007 Chem. Mater. $193472-8$

[22] Maynard, AD, Baron, PA, Foley, M, Shvedova, AA, Kisin, ER and Castranova, V 2004 J. Toxicol. and Environ. Health-Part A 67 (1) 87-107

[23] Senaratne, I and Shooter, D 2004 Atomspheric Environment 38 3049-59

[24] Ely, JC, Neal, CR, Kulpa, CF, Schneegurt, MA, Seidler, JA and Jain, JC 2001 Environ. Sci. Technol. 35 3816-22

[25] Rauch, S, Hemond, HF, Barbante, C, Owari, M, Morrison, GM, PeuckerEhrenbrink, B and Wass, U 2005 Environ. Sci. Technol. 39 8156-62

[26] Klee, RJ and Graedel, TD 2004 Annu. Rev. Environ. Resour. 29 69-107 
[27] Murr, E, Bang, JJ, Esquivel, EV, Guerrero, PA and Lopez, DA $2004 \mathrm{~J}$. Nanoparticle Res. 6 241-51

[28] Reisch, MS and Tullo, AH 2005 Chem. and Eng. News 83 (51) 24-32

[29] Environmental Protection Agency / Department of Justice, http://yosemite.epa.gov/opa/admpress.nsf/ bb1285e857b49ac4852572a00065683f/db490ae06c402d7985257164005d $4 \mathrm{bcc}$ !OpenDocument

[30] Environmental Protection Agency / Department of Justice, http://www.epa/gov/Region8/sf/libby/p082703.html

[31] Harutyunyan, AR, Pradhan, BK, Chang, J, Chen, G, and Eklund, PC $2002 \mathrm{~J}$. Phys. Chem. B, 106 8671-75

[32] Chiang, IW, Brinson, BE, Huang, AY, Willis, PA, Bronikowski, MJ, Margrave, JL, Smalley, RE, and Hauge, RH 2001 J. Phys. Chem. B 105 8297-8301.

[33] Itkis, ME, Perea, DE, Niyogi, S, Rickard, SM, Hamon, MA, Hu, H, Zhao, B, and Haddon, RC 2003 Nano Lett. 3 (3) 309-314

[34] Cao, LM, Zhang, XY, Wang, WK, Zhang, ZL and Zhang, Z 2003 Nanotechnology 14 931-4

[35] Ahn, H-S, Lee, S-C, Han, S, L, K-R and Kim, D-Y 2006 Nanotechnology 17 909-12

[36] Buryskova, B, Hilscherova, K, Blaha, L, Marsalek, B and Holoubek, I 2006 Environ. Toxicol. 21 (6) 590-98

[37] Valley, JW and Cole, DR 2001 Stable Isotope Geochemistry (Washington, DC: Mineralogical Society of America)

[38] Eglinton, TI and Repeta, DJ 2003 Organic Matter in the Contemporary Ocean In Treatise on Geochemistry, vol 6, ed HD Holland and KK Turekian (Morristown, NJ: Elsevier Ltd.)

[39] Peckmann, J and Thiel, V 2004 Chem. Geol. 205 443-67

[40] Abrajano Jr, TA and Yan, B 2003 High Molecular Weight Petrogenic and Pyrogenic Hydrocarbons in Aquatic Environments. In Treatise on Geochemistry, vol 9; ed HD Holland and KK Turekian (Morristown, NJ: Elsevier Ltd.) 
[41] De Santos Neto, EV and Hayes, JM 1999 AAPG Bulletin March, 83(3) 496518

[42] Kirk-Othmer Encyclopedia of Chemical Technology. 2001 (New York, NY: John Wiley \& Sons)

[43] Jonsson, M, Nerushev, OA, Campbell, EEB 2007 Nanotechology 18305702 (DOI:10.1088/0957-4484/18/30/305702)

[44] Zhao, J, Lu, JP, Han, J and Yan, C-K 2003 Appl. Phys. Lett. 82 3746-48

[45] Kuwana, K, Li, TX, and Saito, K 2006 Chem. Eng. Sci. 61 (20) 6718-26

[46] Kim, S-K, Zhang, Y, Teo, KBK, Bell, MS, Gangloff, L, Wang, X, Milne, WI, Wu, J, Jiao, J, and Lee, S-B 2007 Nanotechnology 18185709 (6pp)

[47] Schwarzenbach, RP, Gschwend, PM and Imboden, DM 2003 Environmental Organic Chemistry, $2^{\text {nd }}$ Edition. (New York, NY: John Wiley \& Sons)

[48] Ushida, K, Yshida, Y, Kozawa, T, Tagawa, S and Kira, A 1999 J. Phys. Chem. A 103 4680-89.

[49] Shi Kam, NW, O'Connell, MO, Wisdom, JA and Dai, H 2005 PNAS. 102 (33) 11600-05.

[50] Arnold, WA and Roberts, AL 1998 Environ. Sci. Technol. 32 3017-25.

[51] Scherer, MM, Balko, BA, Gallagher, DA and Tratnyek, PG 1998 Environ. Sci. Technol. 32 3026-33

[52] Frost and Sullivan. 2004 "An Assessment on the Future of Carbon NanotubesStrategic Analysis of the Market \& Potential." (New York, NY: Frost and Sullivan Ltd.)

[53] Linkov, I, Satterstrom, FK, Steevens, J, Ferguson, E and Pleus, RC $2007 \mathrm{~J}$. Nanoparticl Res. 9 (4) 543-54. 


\section{SUPPORTING INFORMATION}

(Note: This information did not appear in or with the original manuscript published by Nanotechnology).

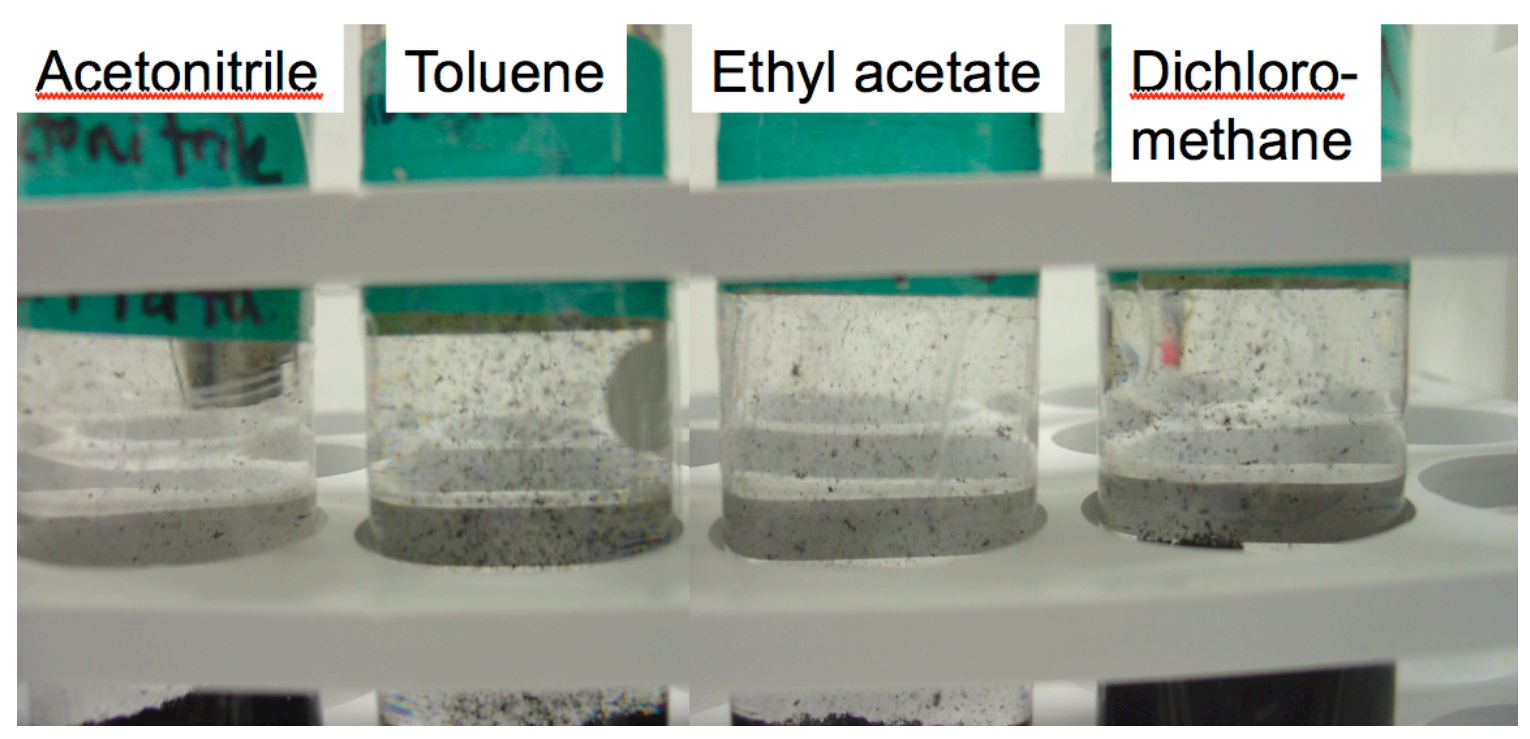

Figure S1. Carbon Nanotech Inc. (HiPCO ${ }^{\circledR}$ CNTs) in various organic solvents in $60-\mathrm{mL}$ glass vials. Roughly equivalent volumes of dry CNTs were added to each vial that contained organic solvent. (Solvent was added to the vial first, then CNTs were introduced to minimize release of the CNTs into the laboratory air). These photographs were taken after CNTs had settled for over 30 minutes. If the vials were agitated, differences between the organic solvent CNT suspensions were indiscernible to the eye. Ethyl acetate was chosen as a carrier solvent, as it had been used historically as a vector for black carbon in our laboratory. Prior to use, the ethyl acetate suspension was vigorously agitated by hand to break up agglomerated CNT particles. For a sense of scale, the approximate diameter of the each vial in this photo is $\sim 2.5 \mathrm{~cm}$. 


\section{CHAPTER 3:}

ANALYSIS OF SINGLE-WALLED CARBON NANOTUBES

IN ENVIRONMENTAL MATRICES

\section{BY THERMOGRAVIMETRY-MASS SPECTROMETRY}

\section{By}

Desirée L. Plata 


\begin{abstract}
Single-walled carbon nanotube (SWCNT) production is expanding and releases of SWCNTs to the environment are expected to increase accordingly. In spite of this rapid development, there are no established methods with which to detect or quantify SWCNTs in environmental matrices (e.g., air or sediment). Thus, there is no way to gauge biological exposure to, or regulate the release of, these novel nanomaterials. SWCNTs have relatively high thermal stabilities, similar to black carbon $(\mathrm{BC})$, and we investigated the use of thermal techniques to isolate and quantify SWCNTs. Test materials included ten types of commercially available SWCNTs, $\mathrm{C}_{60}$ fullerene, multi-walled CNTs, representative biological molecules, BC soot, natural coastal sediments, and SWCNTamended sediments. SWCNTs exhibited a broad range in degradation temperatures that varied with the metal catalyst, and thermal analytical methods may require SWCNT-type specific parameters. To improve identification capabilities of a select SWCNT, gases evolved during thermal treatments were measured by mass spectrometry. SWCNTs produced diagnostic ion ratios. Current detection limits are on the order of $10 \mathrm{ug}_{\mathrm{SWCNT}}$ per sample, and this is set by interfering ions that arise from the instrument's non-airtight design and co-degrading natural materials. While future instrumental modifications may improve this limitation, the current method is sufficient for quantifying SWCNTs in laboratories and industrial sites where SWCNTs are handled, but not adequate for environmental levels of SWCNTs (which we estimate to be less than 10 pg $_{\text {SWCNT }} \mathrm{g}^{-}$ 1 sediment). Nevertheless, the method is able to distinguish between natural (e.g., BC soot) and engineered (e.g., SWCNTs) nanoparticles. Thus, in the case of some SWCNTs, we present a low-cost, high-throughput approach to ensure that the materials are not released from expected point sources at unacceptable levels.
\end{abstract}




\section{INTRODUCTION}

Carbon nanotubes (CNTs) have exceptional electrical, mechanical, and thermal properties. They have generated an impressive list of potential and demonstrated applications ${ }^{1-3}$; and the CNT industry is growing at a remarkable rate, doubling nearly every two years ${ }^{4}$. Although industrially promising, preliminary toxicological studies suggest that powdered CNTs are cytotoxic to alveolar macrophages ${ }^{5,6}$, may result in precancerous, pulmonary granulomas ${ }^{7}$, and adversely affect the life cycle development of marine organisms that play a critical role in nutrient cycling in the upper ocean ${ }^{8}$. Historically, no industrially important chemical has been synthesized, used, and disposed of without some release to the environment (e.g., polycholorinated biphenyls (PCBs), methyl-tert butyl ether (MTBE), and perfluorooctanoic acids (PFOAs)). Thus, we expect a growth in environmental levels of CNTs with the growth of the nanotube industry. Present CNT analytic techniques are limited to electron microscopy (EM) and spectroscopic analyses of small (mg), relatively pure samples (i.e., as-produced SWCNTs that are not mixed with other components). There are no established procedures with which to measure CNTs in complex environmental matrices. Consequently, there is no way to monitor the release of industrially synthesized CNTs or gauge their transport, accumulation, and ultimate biological exposure levels.

Successful isolation and detection of CNTs from environmental mixtures (e.g., air, water, soil, or sediment) will ultimately rely on unique chemical or physical properties of the CNTs that are distinct from other ubiquitous carbon forms. CNTs are

cylinders of $s p^{2}$-hybridized carbon atoms arranged in repeating, aromatic hexagonal rings. While variations in synthetic approaches and post-fabrication handling result in a diverse class of materials ${ }^{9}$, the majority of CNTs will exist as agglomerated, hydrophobic particles $^{10}$. Black carbon soot (BC soot) also forms clusters of hydrophobic nanoparticles, and its aromatic carbon skeleton is chemically similar to that of CNTs. Furthermore, these two materials have similar optical and physical properties (e.g., black color, hydrophobicity, and thermal stability). Methods to measure BC in environmental samples have been under development for approximately 30 years $^{11}$, and a recent 
laboratory inter-comparison ${ }^{12}$ revealed that thermal oxidation techniques (e.g., chemothermal oxidation at $\left.375^{\circ} \mathrm{C}(\mathrm{CTO}-375)^{13}\right)$ successfully isolate $\mathrm{BC}$ soot from other $\mathrm{BC}$ materials, such as wood char. Thus, particles with similar chemical structures and physical properties can be separated and quantified using thermal analysis. Thermal methods (e.g, thermogravimetric analysis (TGA)) are also used to determine the purity of commercial carbon nanotubes, which typically contain several forms of carbon (e.g., single-walled CNTs (SWCNTs), multi-walled CNTs (MWCNTs), "multi-shell” carbon ${ }^{14}$, fullerenes, and amorphous carbon), as well as residual metal catalysts, and there is promise that similar techniques may be used to isolate CNTs in environmental samples.

To establish the feasibility and limitations of such analyses for the detection of CNTs in the environment, we determined the thermal stability of several commercially available SWCNT powders, MWCNTs, fullerenes, representative biological materials (e.g., bovine serum albumin and methylcellulose), and representative environmental mixtures (e.g., urban dust, diesel particulate matter, marine sediments, and SWCNTamended sediments). To improve identification capabilities and potentially reduce detection limits, we monitored evolved gases during temperature-programmed oxidations (TPO) and hydrogen-assisted thermal degradations (HATD) by online mass spectrometry (MS). Using unique mass loss temperatures and evolved gases, we evaluated the limitations and promise of TGA-MS as a tool to separate and quantify several forms of natural and engineered organic matter.

\section{EXPERIMENTAL METHODS}

Samples. SWCNT powders were purchased from multiple manufacturers between September 2005 and March 2006 and were characterized as described in Plata et $a l^{9}$. SWCNT samples contained variable quantities and types of metal catalysts, as outlined in Table 1. Non-SWCNT materials included potentially interfering materials (fullerenes $\left(\mathrm{C}_{60}\right.$, Materials and Electrochemical Research Corporation) and MWCNTs (Nanocyl)), as well a non-interfering representative compounds (methylcellulose). Un- 
amended environmental matrices and mixtures included a sediment sample collected from Dorchester Bay, Massachusetts (as described in Accardi-Dey and Gschwend ${ }^{15}$ ), urban dust (National Institute of Standards and Technology Standard Reference Material (NIST SRM) 1649a), diesel particulate matter (NIST SRM 1650), and chestnut wood char (as described in Hammes et al. ${ }^{12}$ ).

SWCNT-amended sediments were prepared by thoroughly mixing a known mass of SouthWest Nanotechnologies Purified (SWP) SWCNTs to a known mass of presieved, ground, and homogenized Dorchester Bay sediment (DBS). Less-concentrated sediment samples were prepared by serial dilutions of this initial stock with un-amended DBS.

Table 1. Single-walled carbon nanotubes used in this study

\begin{tabular}{|c|c|c|}
\hline SWCNT supplier name $^{\mathrm{a}}$ & Symbol $^{b}$ & Metal content ${ }^{\mathrm{c}}(\mathrm{wt} \%)$ \\
\hline SES Research & SES & $0.47 \mathrm{Fe}, 2.8 \mathrm{Co}, 0.69 \mathrm{Mo}$ \\
\hline Carbon Solutions Inc. & CSI & $22.4 \mathrm{Ni}, 6.0 \mathrm{Y}$ \\
\hline CarboLex, Inc. & CLex & $24.3 \mathrm{Ni}, 5.0 \mathrm{Y}$ \\
\hline $\begin{array}{l}\text { Materials \& Electrochemical } \\
\text { Research Corp. }\end{array}$ & MER & $3.53 \mathrm{Ni}, 10.5 \mathrm{Co}$ \\
\hline Helix Material Solutions & Helix & $15.3 \mathrm{Ni}, 5.3 \mathrm{Y}$ \\
\hline Manufacturer $X^{\mathrm{d}}$ & ManX & $1.21 \mathrm{Fe}, 0.08 \mathrm{Mo}$ \\
\hline Nanocs Inc. & Nanocs & $20.3 \mathrm{Ni}, 4.2 \mathrm{Y}$ \\
\hline $\begin{array}{l}\text { Nanostructured \& Amorphous } \\
\text { Materials }\end{array}$ & NanoAm & $0.24 \mathrm{Fe}, 2.87 \mathrm{Co}, 1.00 \mathrm{Mo}$ \\
\hline $\begin{array}{l}\text { SouthWest NanoTechnologies } \\
\text { Inc. (SWeNT, Inc.) }\end{array}$ & SWP & $0.07 \mathrm{Fe}, 4.1 \mathrm{Co}, 6.9 \mathrm{Mo}$ \\
\hline Carbon Nanotechnologies Inc. & $\mathrm{CNI}$ & $22 \mathrm{Fe}$ \\
\hline \multicolumn{3}{|c|}{ 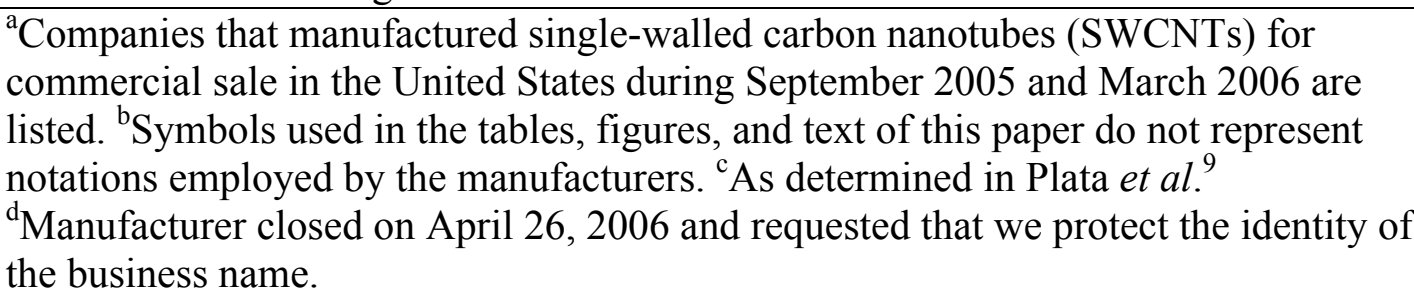 } \\
\hline
\end{tabular}

Thermogravimetric analysis-mass spectrometry. TPOs and HATDs were performed using a TA Instruments Q50 thermogravimetric analyzer (TGA) interfaced to a Pfeiffer ThermoStar quadrupole mass spectrometer $\left(\mathrm{MS}\right.$; heated $\left(200^{\circ} \mathrm{C}\right)$ quartz 
capillary transfer line, CH-TRON detector with secondary electron multiplier; 1-300 amu possible scan range; sampling rate of 2 standard cubic centimeters per minute). Sample sizes were between 3-3.3 mg for relatively pure samples (i.e., non-sedimentary material) and between 40-44 mg for environmental matrices (e.g., sediments and soils). Oxygenassisted degradations in dry air $\left(100 \mathrm{~mL} \mathrm{~min}^{-1}\right)$ were heated to $80^{\circ} \mathrm{C}$, held for $10 \mathrm{~min}$ (to stabilize the temperature), ramped at $5^{\circ} \mathrm{C} \min ^{-1}$ to $900^{\circ} \mathrm{C}$, and held for $60 \mathrm{~min}$. HATDs in ultra high purity hydrogen $\left(10 \mathrm{~mL} \mathrm{~min}^{-1}\right)$ were heated to $80^{\circ} \mathrm{C}$, held for $60 \mathrm{~min}$, ramped at $5^{\circ} \mathrm{C} \min ^{-1}$ to $900^{\circ} \mathrm{C}$, and held for $60 \mathrm{~min}$. Ion currents were collected for 60 seconds and scanned in sequence for $\mathrm{m} / \mathrm{z} 2,4,12,14,16,18$, and 20-79. Between runs, platinum sample pans were washed with $25 \%(\mathrm{v} / \mathrm{v}) \mathrm{HNO}_{3}$ (trace metal grade), rinsed with copious Milli-Q water (18 M $)$, and then combusted at $900^{\circ} \mathrm{C}$ in air for at least 1 hour.

Data processing. Mass spectral data was normalized and/or background subtracted as described below, depending on particular ion interferences that arise from choice of carrier gas.

Correction for TPOs: To account for instrumental drift between each sample analysis, each ion ratio $\left(\mathrm{X}_{\text {sample }} / \mathrm{Y}_{\text {sample, e.g., }} 18_{\text {sample }} / 44_{\text {sample }}\right)$ was normalized to an arbitrarily selected standard run using the ratio of the average ion current $\mathrm{m} / \mathrm{z} 28$ ( $28_{\text {sample }}$ ) in the sample to the average ion current $\mathrm{m} / \mathrm{z} 28$ in the standard (28 $8_{\text {standard }}$ (Eqn 1).

$$
\left(\frac{18_{\text {sample }}}{44_{\text {sample }}}\right)_{\text {corrected }}=\left(\frac{18_{\text {sample }}}{44_{\text {sample }}}\right) \times\left(\frac{28_{\text {sample }}}{28_{\text {standard }}}\right)
$$

Correction for HATDs: Our particular TGA-MS was not designed to be airtight (although airtight models are available), and small air leaks in the system gave relatively high background signals due to $\mathrm{N}_{2}, \mathrm{O}_{2}$, and $\mathrm{Ar}$ (ion currents $28,32,40$, respectively). The $\mathrm{N}_{2}$ and $\mathrm{O}_{2}$ interferences were not improved by placing the TGA-MS in a sealed Arfilled chamber. Ethene (ion current $\mathrm{m} / \mathrm{z} 28$ ) is an important mass formed by carbon nanotube (CNT) reaction with $\mathrm{H}_{2}$, and to lower interference from $\mathrm{N}_{2}$, the ion current $\mathrm{m} / \mathrm{z}$ 
28 for each sample was background subtracted. The correction was performed by subtracting the average ion current $\mathrm{m} / \mathrm{z} 28$ during a "blank" segment (no sample degradation) of each analysis ( $28_{\text {background }}$ ), normalized for signal drift using ion current $\mathrm{m} / \mathrm{z} 40$, from the ion current $\mathrm{m} / \mathrm{z} 28$ during the rest of the thermal program ((28 sample $)$ Eqn 2).

$$
28_{\text {sample,subrracted }}=28_{\text {sample }}-28_{\text {background }}\left(\frac{40_{\text {sample }}}{40_{\text {background }}}\right)
$$

Ion ratios were then normalized as described by Eqn 1.

Blanks and precision of mass and temperature. Several materials that were expected to be free of organic carbon were analyzed. These included pre-combusted and ground (to a fine, sediment-like powder) Ottawa quartz sand, pre-combusted quartz fiber filters (QFFs), and pre-combusted and acid-washed platinum sample pans. The oxidation temperature was reproducible to within $0.1^{\circ} \mathrm{C}$, and the mass was precise to $0.01 \mathrm{mg}$.

\section{RESULTS AND DISCUSSION}

When subjected to an increasing temperature program in a reactive atmosphere, carbonaceous materials lose mass at characteristic (relative) temperatures, which are reflective of their structures and potentially other factors (e.g., metals mixed with the material). Each mass loss event ("peaks" in a plot of differentiated mass loss) can then be attributed to a distinct phase in the sample.

TPO of SWCNTs and natural organic materials. SWCNTs exhibited a diverse range of oxidative temperatures, from $375^{\circ} \mathrm{C}$ to $540^{\circ} \mathrm{C}$, and varying proportions of carbonaceous impurities (Figure 1). The majority of SWCNT powders produced two oxidation peaks: (1) a lower thermal stability signal that is conventionally presumed to be due to the oxidation of amorphous carbon and (2) a higher thermal stability signal that is conventionally presumed to be due to the oxidation of SWCNTs. In some cases (e.g., 
SES), contaminant phases were not amorphous carbon, but instead, higher thermal stability material, such as MWCNTs or multi-shell carbon (i.e., soot). In other cases (e.g., Nano Am., MER, and potentially CNI and ManX), only one phase was readily apparent in the thermogram, and this could be the result of either very pure SWCNTs or the co-oxidation of multiple phases. All mass losses were due to the oxidation of carbonaceous material (as confirmed by MS and discussed later), except in the case of the volatilization of molybdenum oxides (around $690^{\circ} \mathrm{C}$ ) from the SWP sample.

Several factors can influence the apparent thermal stability of a material, including the heating rate, oxygen supply, and oxygen's access to the carbon surfaces (e.g., surface area and material packing ${ }^{16,17}$ ). The temperature program, sample size, and bulk oxygen supply were well controlled in these experiments, and the observed differences in SWCNT oxidation temperatures must be due to some intrinsic property (or properties) of the SWCNTs. Several researchers ${ }^{14,18}$ have noted that the SWCNT oxidation temperature is inversely related to the total metal content, suggesting that metals may catalyze the oxidation. Presumably, this catalysis results from the formation of metal oxides that generate concentrated microenvironments of oxygen in close proximity to the SWCNT lattice $^{19}$. However, it is also possible that different catalytic metals may produce SWCNTs with distinct properties (e.g., average surface area of SWCNTs) that influence the oxidation temperatures of the nanotube products. While there was a very loose relationship with metal content and the oxidation temperature (in the data reported here, $n$ $=10$, correlation coefficient of 0.28 ), the type of metal mixture was critically important to the oxidation temperature of the SWCNTs (Figure 2). For example, all Ni/Y catalyzed SWCNTs had maximum thermal oxidation temperatures around $420^{\circ} \mathrm{C}\left(413-428^{\circ} \mathrm{C}\right.$, excluding the CSI sample, which has a relatively complex thermogram), while $\mathrm{Fe} / \mathrm{Co} / \mathrm{Mo}$ catalyzed samples exhibited much higher thermal stabilities (between 510 and $540^{\circ} \mathrm{C}$ ). Furthermore, in spite of a large spread in Ni content (range of $10 \mathrm{wt} \%$ ) of Ni/Y catalyzed samples, the oxidation temperatures were fairly consistent between samples. Thus, metal concentration did not significantly impact the oxidation temperature of SWCNTs. To our knowledge, there has been no systematic study of metal catalyzed oxidation of 

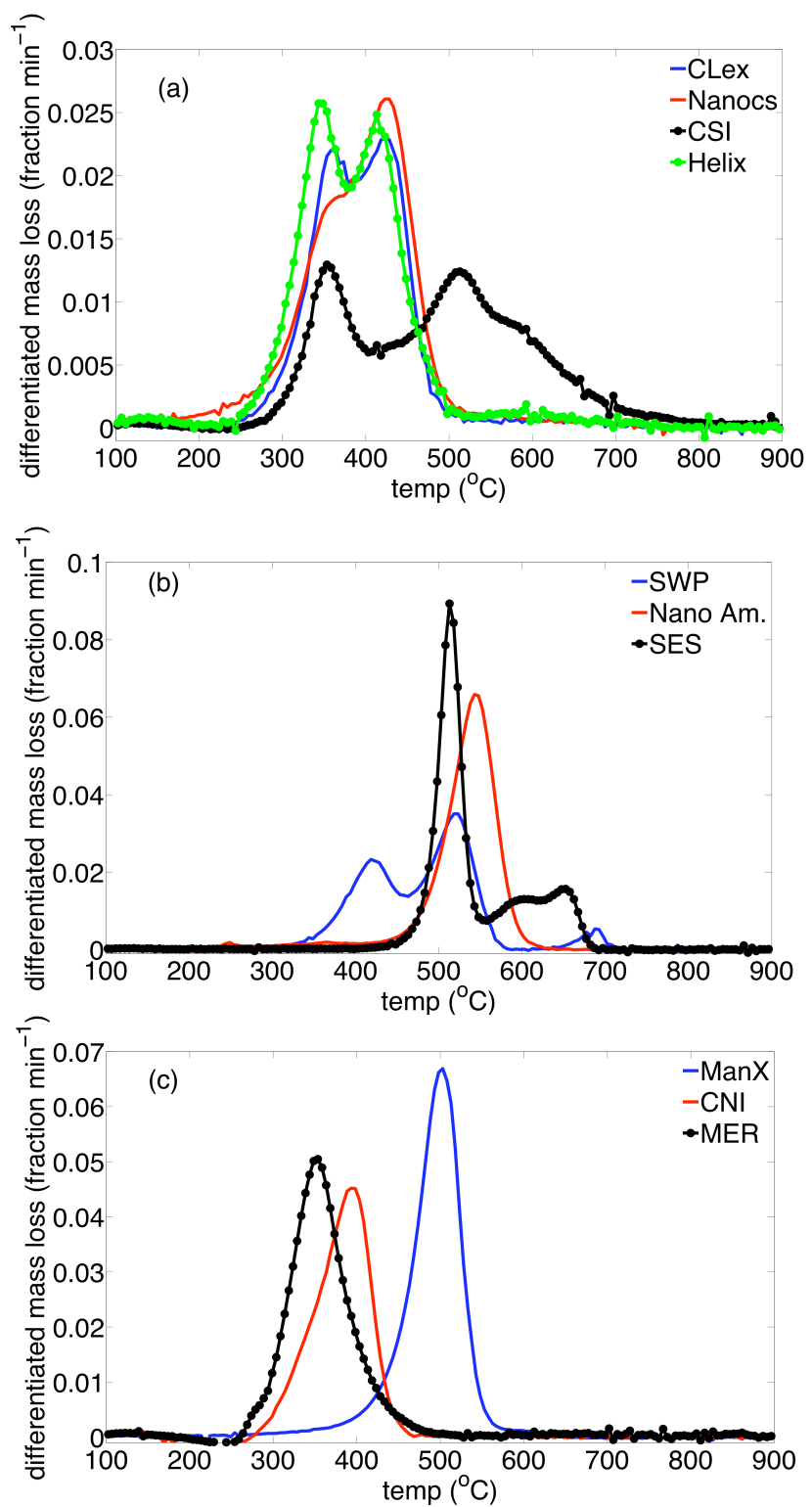

Figure 1. Differentiated oxidation thermograms of SWCNT powders. Each SWCNT sample is catalyzed with either (a) Ni and Y, (b) Fe, Co, and Mo, or (c) Fe and Mo (Man $\mathrm{X}), \mathrm{Fe}(\mathrm{CNI})$, or Ni and Co (MER). Multiple mass loss peaks are due to the oxidation of different phases, which are all carbonaceous (except for the highest thermal stability peak in the SWP sample, which is due to the loss of molybdenum oxides). For thermograms with two major peaks, the lower stability peak was attributed to amorphous carbon and the higher thermal stability peak was attributed to SWCNTs, except in the case of SES. 
recalcitrant carbon forms. However, $\mathrm{NiO}$ is known to be an efficient oxidation catalyst ${ }^{20}$, and others have shown that different transition metals have distinct effects on the degradation of test materials (e.g., $\mathrm{S}_{2} \mathrm{O}_{8}{ }^{2-}$-mediated metal catalyzed oxidation of peptides (with $\mathrm{Fe}, \mathrm{Co}, \mathrm{Ni}$, or $\mathrm{Mn})^{21}$ or the oxidation of atmospheric sulfur by $\mathrm{Mn}$ or $\mathrm{Fe}^{22}$ ).

While maximum oxidation temperatures are relatively consistent between samples with similar catalytic metal composition, the wide distribution in SWCNT thermal stability across catalyst types demonstrates that no single thermal oxidation technique will quantify all SWCNTs. Indeed, the need for "compound-specific" analytical techniques exists for many anthropogenic pollutants (e.g., PCB congeners have distinct gas chromatographic retention times and/or molecular ions). Knowing the specific type of SWCNT likely to be present in a field or lab sample (e.g., Ni- vs. Co- catalyzed), one could tailor the analytical method to isolate and quantify the desired variety of SWCNTs. Here, we will focus on developing techniques to isolate the SWP nanotubes, which have been selected as a standard material for SWCNTs by NIST ${ }^{\mathrm{a}}$. This approach requires that there is minimal overlap in the thermal stability of SWP SWCNTs and other natural materials.

Several representative "natural" materials were chosen, including samples that contain no BC or SWCNT (i.e., a carbohydrate, methylcellulose, and a protein, bovine serum albumin) and samples that contain different types of BC (i.e., wood char and diesel particulate matter) (Figure 3). Methylcellulose had a low thermal stability that did not interfere with SWP, which is promising for the analysis of CNTs on cellulose filters. Bovine serum albumin (BSA), on the other hand, had a broad oxidation range that interfered with SWP. Molecules with high nitrogen contents have a tendency to char, forming higher stability materials during the thermal treatment. Visible charring was evident following BSA oxidation, and we expect that environmental matrices with high protein contents will suffer from significant interference. These may be overcome with

\footnotetext{
${ }^{a}$ Note that this designation was made in July 2008, whereas our SWP nanotubes were purchased in January 2007. SWeNT, Inc. may have modified their production process between these two dates.
} 
tandem analyses (e.g., TGA-MS) that enable the distinction between evolved gases due to char-derived $\mathrm{BC}$ and native $\mathrm{BC}$ in the sample. Natural $\mathrm{BC}$ in a sample matrix may exist in several forms, including wood char or BC soot. Wood char had a lower thermal stability than SWP, likely due to greater oxygen access resulting from the porous structure of char, yet there was slight interference with the oxidation onset of the SWCNTs. BC soot, on the other hand, was thermally distinct from SWP. The higher stability of the former is likely due to the multi-layer structure of aromatic sheets present in $\mathrm{BC}$ soot spheres ${ }^{23}$, which limits the oxygen access to the dense interior of the nanoparticles and increases their ultimate oxidation temperature. In contrast, SWCNTs are made up of hollow cylinders that have higher surface-area-to-carbon ratios. This structural difference between BC and SWCNTs may provide an opportunity to distinguish the two materials using thermal techniques.

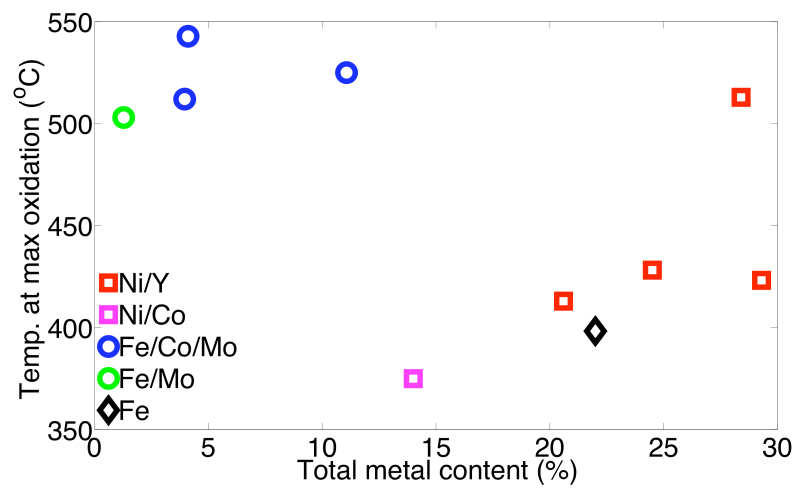

Figure 2. Temperature of maximum SWCNT mass loss rate as a function of total metal and type of metal. The metal content of each SWCNT powder is detailed in Table 1. 

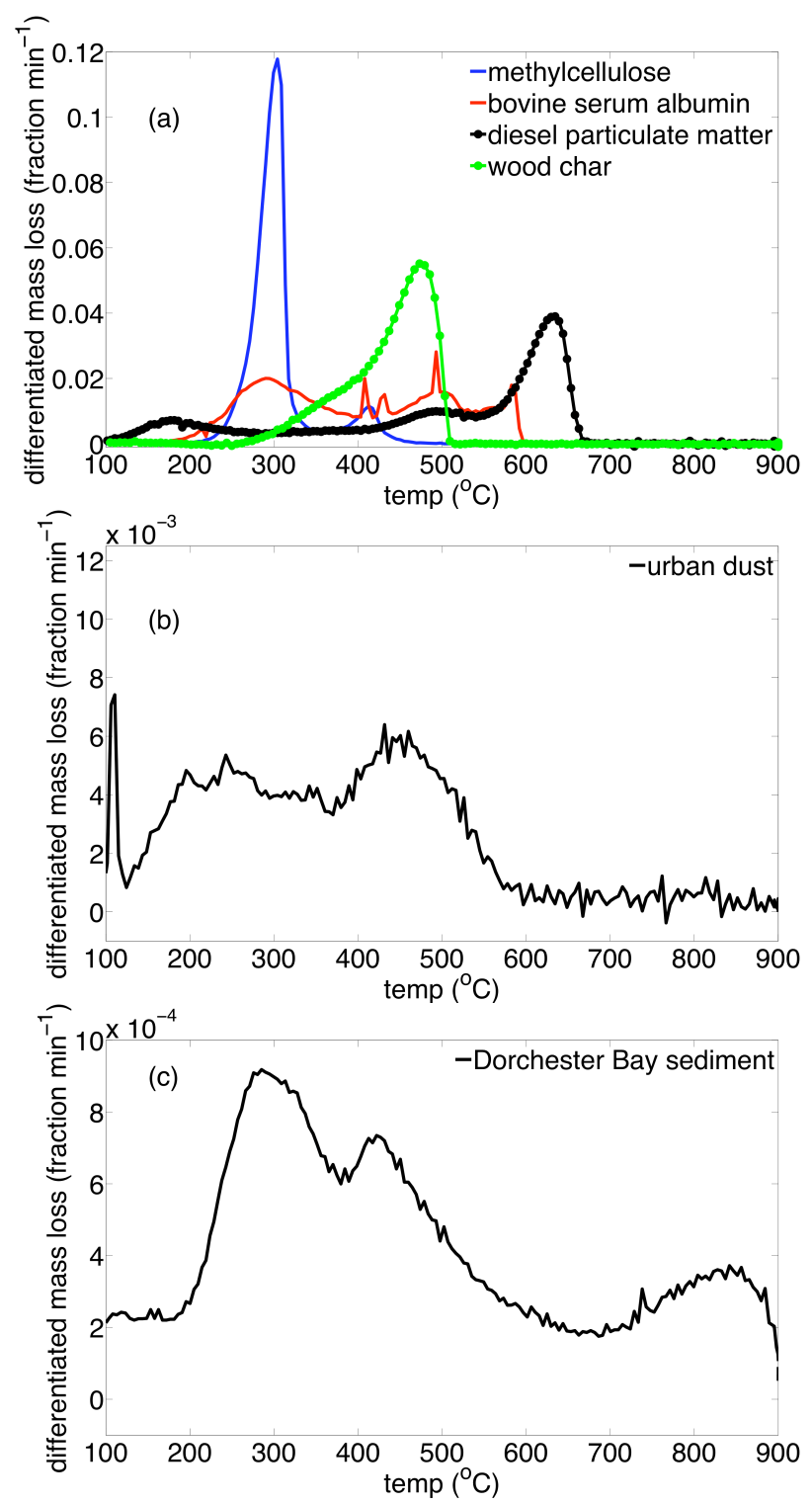

Figure 3. Differentiated oxidation thermograms of representative materials that are likely to be found in the environment. (a) Relatively pure test materials exhibited a wide range of oxidation temperatures, some of which overlapped with SWCNTs. (b, c) Environmental matrices, such as urban aerosol concentrates and sediments, had broad mass loss peaks that would obscure some, but not all, SWCNT oxidation temperature ranges. Thermograms for $\mathrm{C}_{60}$ and MWCNTs are available in the Supporting Information. 
TPO of complex environmental matrices. In more complex environmental matrices, a broad range of material exists, and full resolution of the diverse carbon phases may not be possible using thermal methods alone. Aerosol concentrates of urban dust had significant mass loss throughout the thermogram to $600^{\circ} \mathrm{C}$, co-oxidizing with most SWCNTs (Figure 3b). These interferences likely arose from the presence of recalcitrant chemicals and particles derived from incomplete combustion processes in urban environments, such as automobile exhaust and industrial emissions. Sediment collected from Dorchester Bay, MA had a thermal distribution consistent with the presence of less combustion-derived, high-thermal stability material (Figure 3c). As a result, there was less interference with SWP SWCNTs. Nevertheless, the co-oxidizing phases were nonnegligible, and an additional dimension of information, chemical composition, was sought to improve detection limits of SWCNTs in environmental mixtures.

TPO-MS: Unique ion ratios from SWCNTs. Using mass spectral analysis of the gases evolved during the thermal program, we identified important masses associated with each compound class. An expected combustion product, $\mathrm{CO}_{2}(\mathrm{~m} / \mathrm{z} 44)$, was produced from all test materials (Supporting Information). (Similarly, CO (m/z 28) was evident when the carrier gas was a $\mathrm{He} / \mathrm{O}_{2}(80: 20 \% \mathrm{v} / \mathrm{v})$ mixture, as opposed to dry air, which has an interfering signal due to $\mathrm{N}_{2}$ in air $(\mathrm{m} / \mathrm{z} 28)$ ). The oxidation of methylcellulose and BSA also formed $\mathrm{H}_{2} \mathrm{O}\left(\mathrm{m} / \mathrm{z}\right.$ 18) and either $\mathrm{NO}$ and/or $\mathrm{CH}_{2} \mathrm{O}(\mathrm{m} / \mathrm{z}$ 30). This is consistent with the appreciable $\mathrm{H}, \mathrm{O}$, and $\mathrm{N}$ contents of these materials. In contrast, oxidation of $\mathrm{BC}$ soot and SWCNTs produced no measurable $\mathrm{H}_{2} \mathrm{O}, \mathrm{NO}$, or $\mathrm{CH}_{2} \mathrm{O}$. Historically, geochemists have taken advantage of these differences in chemical structure to identify distinct materials in complex mixtures. For example, $\mathrm{BC}$ soot, with very few functional groups, has $\mathrm{O} / \mathrm{C}$ and $\mathrm{H} / \mathrm{C}$ ratios below 0.3 , whereas most chars have $\mathrm{O} / \mathrm{C}$ ratios from $0.3-0.5$ and $\mathrm{H} / \mathrm{C}$ ratios from $0.3-0.75^{24}$. Thus, the two closely related materials can be distinguished with elemental analysis, and it is possible that SWCNT and $\mathrm{BC}$ may be identified by analogous means.

The variability in H-to-C and O-to-C ratios in natural materials can be represented in a van Krevelen diagram (a plot of $\mathrm{H} / \mathrm{C}$ ratios vs. $\mathrm{O} / \mathrm{C}$ ratios, originally employed for 
the study of coals), where materials extremely depleted in $\mathrm{H}$ and $\mathrm{O}$ are considered $\mathrm{BC}$ or soot-like. Using a similar approach, we invetigated the mass spectral signals evolved during the oxidation of native coastal sediments and SWCNT-amended sediments. Depletions in the ion ratios 18/44 (i.e., $\mathrm{H}_{2} \mathrm{O} / \mathrm{CO}_{2}$ ) and 30/44 (i.e., $\mathrm{CH}_{2} \mathrm{O}$ or $\mathrm{NO} / \mathrm{CO}_{2}$ ) were observed and mapped in van Krevelen space (Figure 4). As expected, pure SWCNTs had extremely low levels of ion ratios 18/44 and 30/44 relative to native sediments. As the SWCNT content of the amended sediment increased, there were measurable reductions in ion ratios $18 / 44$ and 30/44, which were detectable at concentrations as low as $30 \mathrm{ug}_{\mathrm{SWCNT}} \mathrm{g}^{-1}$ sediment.

While these detection limits are sufficient for many laboratory-based experiments and indoor air concentrates where SWCNTs are being handled or produced $\left(50 \mathrm{ug} \mathrm{m}^{-3}\right)^{25}$ they are not sensitive enough to measure SWCNTs in environmental samples. Natural abundances of SWCNTs are estimated to be on the order of $\mathrm{pg} \mathrm{g}^{-1}$ sediment, $\mathrm{pg} \mathrm{m}^{-3}$ air, and less than one $\mathrm{pg} \mathrm{L}^{-1}$ water presently ${ }^{26}$, six orders of magnitude below current TGA-MA detection capabilities. The current detection limit for SWCNTs is controlled by baseline $\mathrm{CO}_{2}$ that is derived from natural sedimentary organic matter and $\mathrm{CO}_{2}$ leaks. Attempts to circumvent this enhanced background signal were unsuccessful. First, we used an altered thermal programs designed to degrade non-SWCNT material while preserving SWCNTs (e.g., rapid heating to and prolonged stasis at a temperature close to, but below, SWCNT degradation temperature), but this did not reduce the interference (Supporting Information). Second, we hypothesized that a multi-dimension approach could offer improved detection limits (e.g., TGA-isotope ratio MS (TGA-IRMS)). Plata et al. ${ }^{9}$ demonstrated that SWP SWCNTs have a depleted ${ }^{13} \mathrm{C}$ signature $\left(\delta^{13} \mathrm{C}_{\mathrm{SWP}}=-49.8 \%\right.$ oo relative to most marine sedimentary organic matter $\left(\delta^{13} \mathrm{C}_{\text {Dorchester Bay Sediment }}=-20.4 \%\right.$ (Supporting Information)). Evolved gases from thermal analysis could be cryogenically 

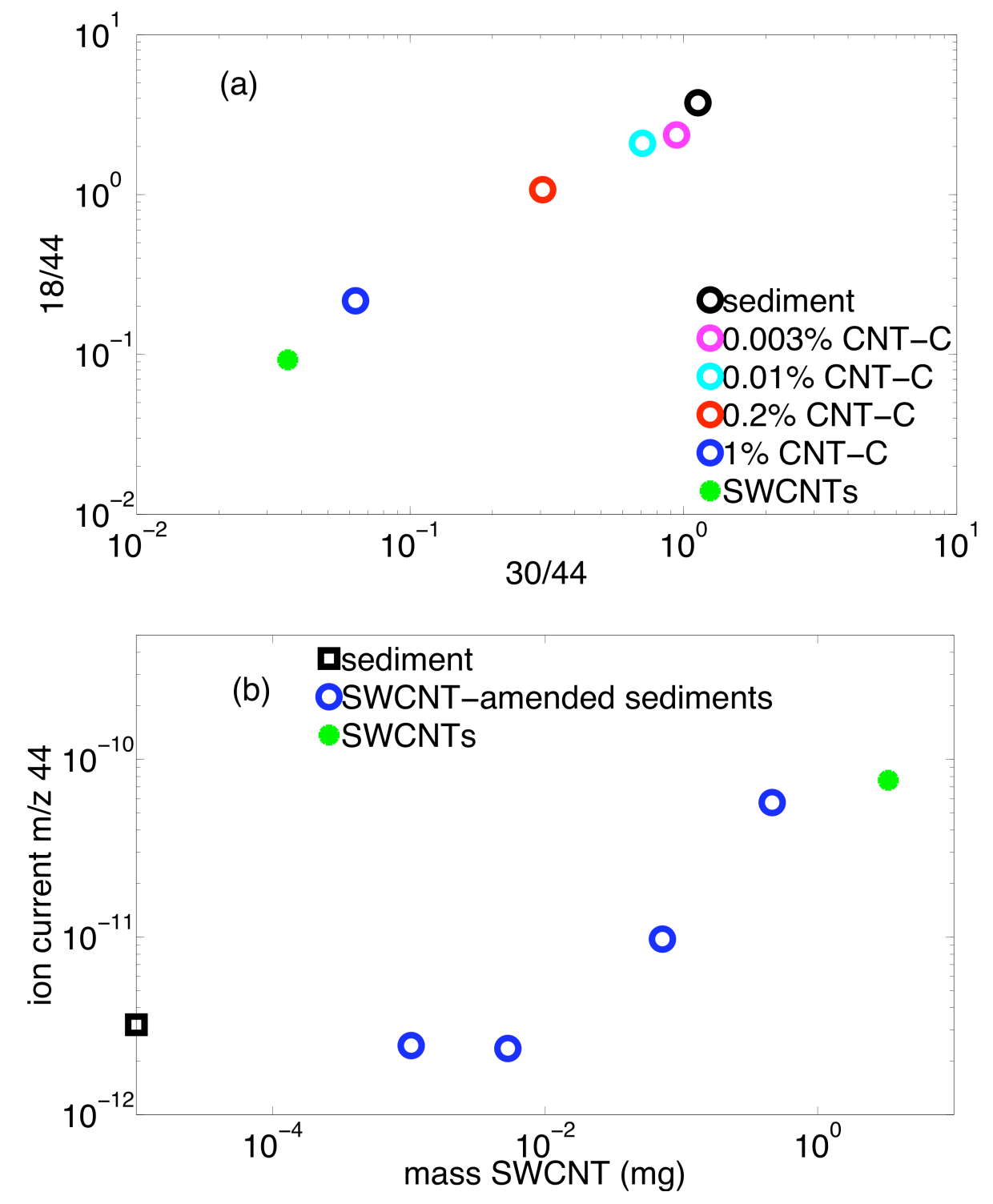

Figure 4. Diagnostic ion ratios observed in thermal oxidations of SWCNT-amended coastal sediments. Increasing amounts (weight percent indicated in figure legend) of SWCNTs were added to sediment collected from Dorchester Bay, MA. (a) When oxidized, pure SWCNTs yielded very little mass fragment 18 (due to $\mathrm{H}_{2} \mathrm{O}$ ) and mass fragment 30 (due to $\mathrm{NO}$ or $\mathrm{CH}_{2} \mathrm{O}$ ), and depletions in the 18/44 and 30/44 ion ratios were observed with increasing SWCNT content of the sediments. (b) The lower bound on SWCNT detection was set by co-oxidizing carbonaceous material, which generated $\mathrm{CO}_{2}$ and increased the baseline signal of ion current $\mathrm{m} / \mathrm{z} 44$. 
focused during the relevant thermal regime and subsequently analyzed (either off or online) by IRMS. The isotopic content of the isolated material would reflect contributions of background organic matter in the sediment as well as the SWCNTs, and the proportion of SWCNTs could be constructed mathematically. Such a calculation would require some knowledge of the ${ }^{13} \mathrm{C}$ content of the organic material in the sediment that remained at high temperature. This stable material is typically petrogenic or pyrogenic (petroleum or fire-derived, respectively) in origin, with $\delta^{13} \mathrm{C}$ values between $25--27 \%{ }^{27}$ (Supporting Information). Using this assumption and considering that interfering material could be up to $0.1 \%$ of the mass of the original sediment (based on typical BC levels in coastal sediments) ${ }^{13}$, we determined that the detection limit of such an approach would be approximately 100 ug $_{\text {SwCNT }} \mathrm{g}^{-1}$ sediment; no improvement on the current tandem analysis (TGA-MS).

As $\mathrm{CO}_{2}$ is a generic product of oxidation of organic carbon, it will likely present a barrier to the detection of a trace contaminant in any sample matrix (i.e., air, water, sediment, or soil). Therefore, we investigated the use of an alternate reactive gas, $\mathrm{H}_{2}$ instead of $\mathrm{O}_{2}$, to potentially produce SWCNT-specific masses.

\section{HATD of SWCNTs, natural organic materials, and complex environmental}

matrices. Heating SWCNTs in pure hydrogen, rather than dry air, increased the maximum degradation temperature of all SWCNTs (Figure 5). In some cases, the thermal program did not reach high enough temperatures to degrade the SWCNTs (ManX, CLex) or associated contaminants (SES), in spite of a prolonged exposure (60 $\mathrm{min}$ ) to $\mathrm{H}_{2}$ at $900^{\circ} \mathrm{C}$. In addition to shifts in the thermal stability, there was increased resolution of previously co-oxidizing phases in some samples (MER, which is known to contain fullerenes and MWCNTs, and NanoAm.). Phases that were not observed in TPOs became apparent in HATDs, and this demonstrates that the use of TPO alone is not sufficient to determine the purity of SWCNTs. TPO is often used exclusively to determine the quantity of carbonaceous and metallic impurities in SWCNTs in industry ${ }^{28}$,

materials engineering ${ }^{18}$, and even environmental chemistry ${ }^{19}$ (where low thermal stability peaks are considered amorphous carbon and the mass remaining at the end of the TPO is 

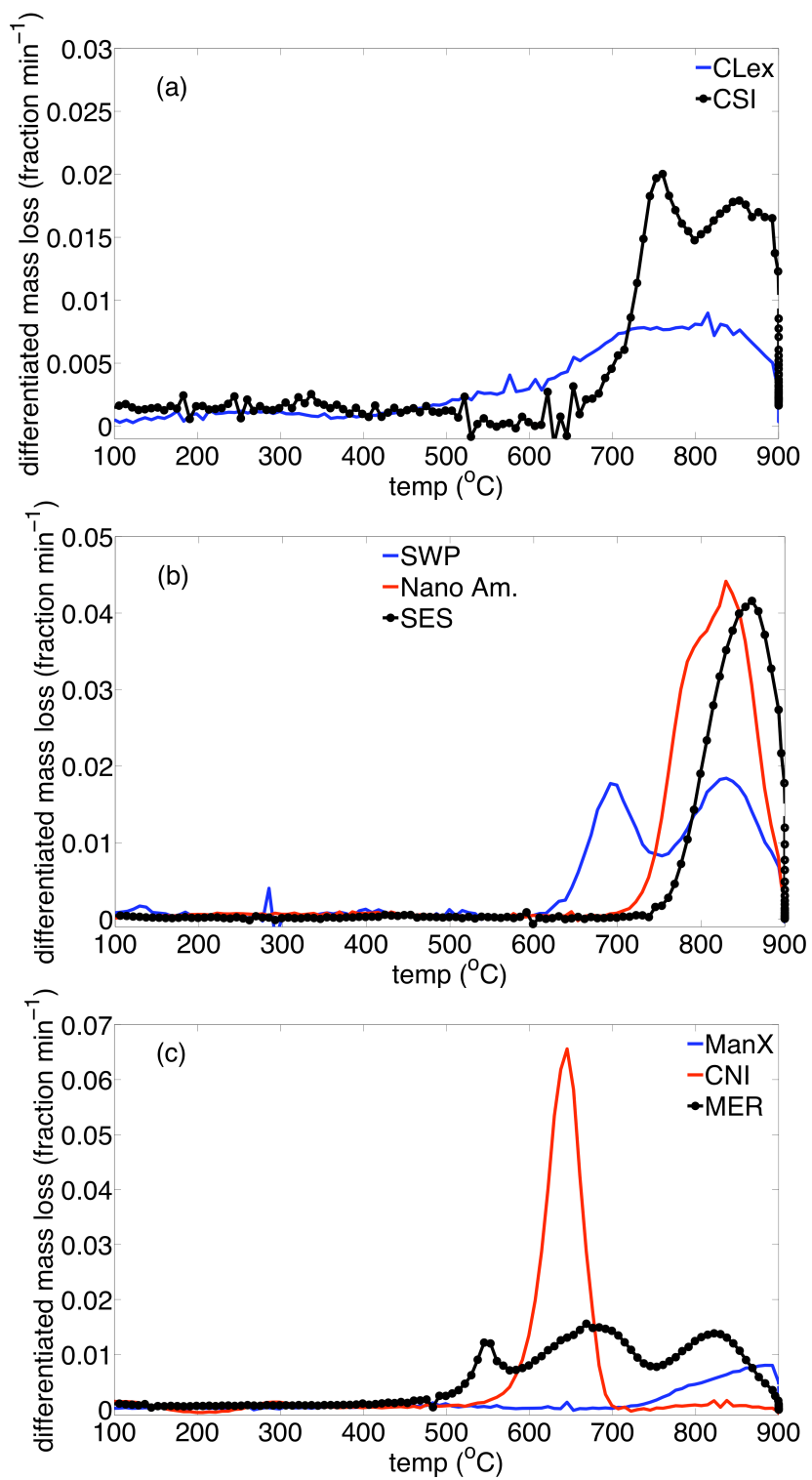

Figure 5. Differentiated thermograms of SWCNT powders subjected to hydrogenassisted thermal degradation. Each SWCNT sample is catalyzed with either (a) Ni and Y, (b) Fe, Co, and Mo, or (c) Fe and Mo (Man X), Fe (CNI), or Ni and Co (MER). Multiple mass loss peaks are due to the degradation of different phases, which are all carbonaceous. Some phases that were not observed in the TPOs are apparent during HATDs (e.g., MER, NanoAm). After 60 min of thermal decomposition in $\mathrm{H}_{2}$ at $900^{\circ} \mathrm{C}$, some material in the SWCNT powders was not fully degraded. 
considered to be metal oxide. While the approach appears to be valid in some cases (e.g., for the CNI sample), TPO does not necessarily separate all carbon-based SWCNT coproducts.

In addition to offering increased phase resolution in SWCNT powders, HATD reduces interferences from natural materials (Figure 6). Methylcellulose and BSA's principle mass losses occur below 350 and $450^{\circ} \mathrm{C}$, respectively, well below SWCNT degradation temperatures. As in TPO, BSA charred into high thermal stability material(s), and $20 \%$ of the initial mass remained after the HATD. This material was at least as stable as $\mathrm{BC}$ soot, which did not degrade below $900^{\circ} \mathrm{C}$. Diesel particulate matter only experienced a very small mass loss during HATD, and this was due to low thermal stability organic compounds associated with the BC particles. As SWP SWCNTs degraded between 750 and $900^{\circ} \mathrm{C}$, they can be separated from BC soot using HATD. However, interferences from complex environmental mixtures persisted. In the case of urban dust, mass losses overlapped with those of SWP SWCNTs to a greater extent than under TPO. Coastal sediments had almost identical co-degradation in the thermal region of SWP degradation during both TPO and HATD. In order to overcome this limitation, diagnostic masses from SWCNT degradation were sought to identify the materials in natural environments.

HATD-MS: Unique ion ratios from SWCNTs. The mass spectra of evolved gases during HATD showed that all test materials produced ethene (m/z 28) (Supporting Information). Methylcellulose and BSA also produced $\mathrm{H}_{2} \mathrm{O}(\mathrm{m} / \mathrm{z} 18)$, reflecting the enriched oxygen content of these materials relative to SWCNTs and MWCNTs, which produced no detectable $\mathrm{H}_{2} \mathrm{O}$. There were few other diagnostic masses that were produced by the HATD of the test materials, but SWCNTs degraded at rates that were sufficient to draw down the signal from the reactant gas, $\mathrm{H}_{2}(\mathrm{~m} / \mathrm{z} 2)$. This consumption of $\mathrm{H}_{2}$ and depletion of $\mathrm{H}_{2} \mathrm{O}$ was detectable in SWCNT-amended sediments down to (at least) 100 $u_{\text {SWCNT }} \mathrm{g}^{-1}$ sediment $($ Figure 7). 

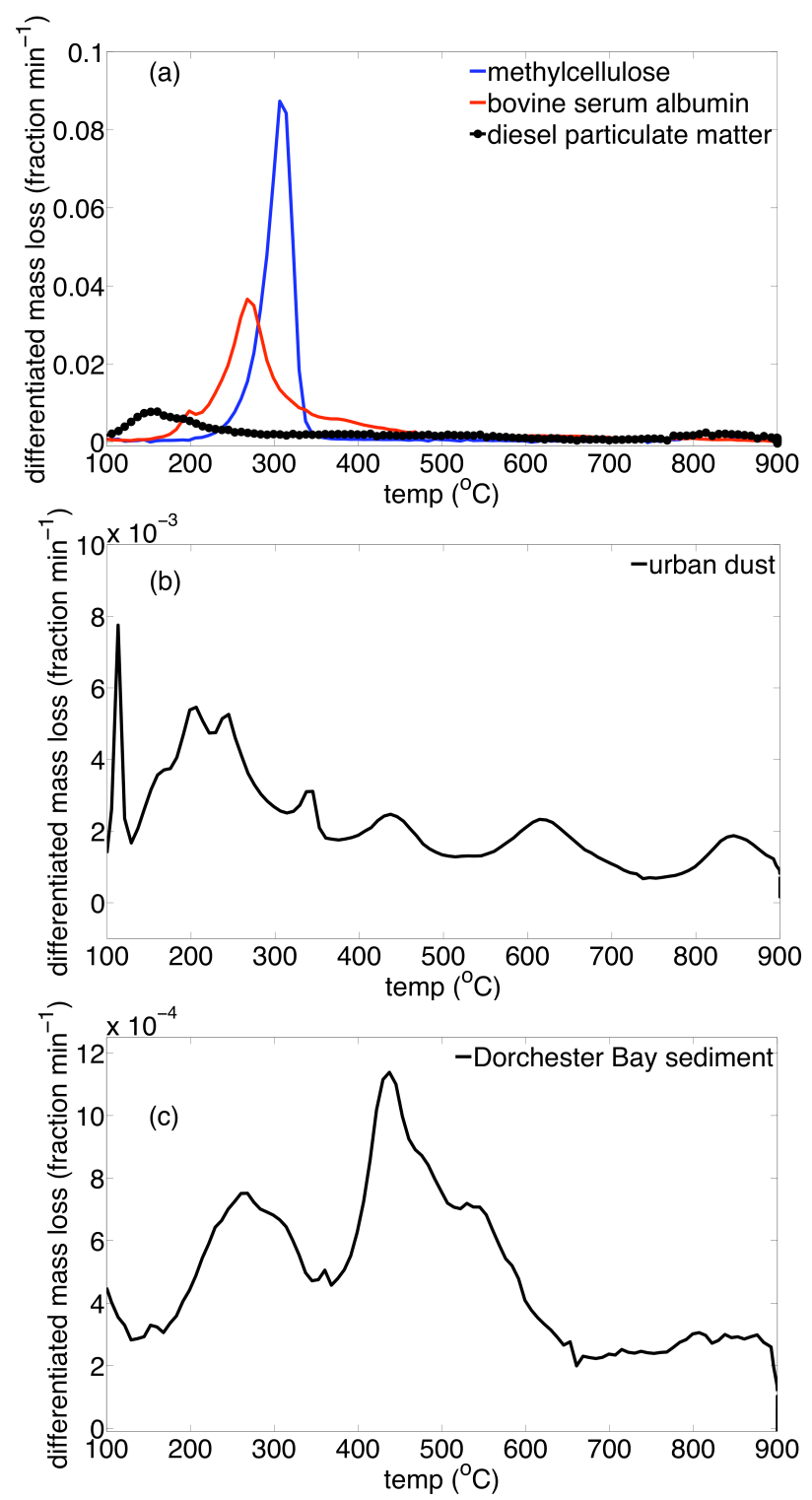

Figure 6. Differentiated hydrogen-assisted thermograms of representative materials that are likely to be found in the environment. (a) Relatively pure test materials exhibited a wide range of degradation temperatures. Natural materials did not overlap with the mass loss of SWCNTs. (b, c) Environmental matrices, such as urban aerosol concentrates and sediments, had broad mass loss peaks. The sediment sample exhibited reduced interference with SWCNT degradation ranges in HATD. Thermogram for MWCNTs are available in the Supporting Information. 

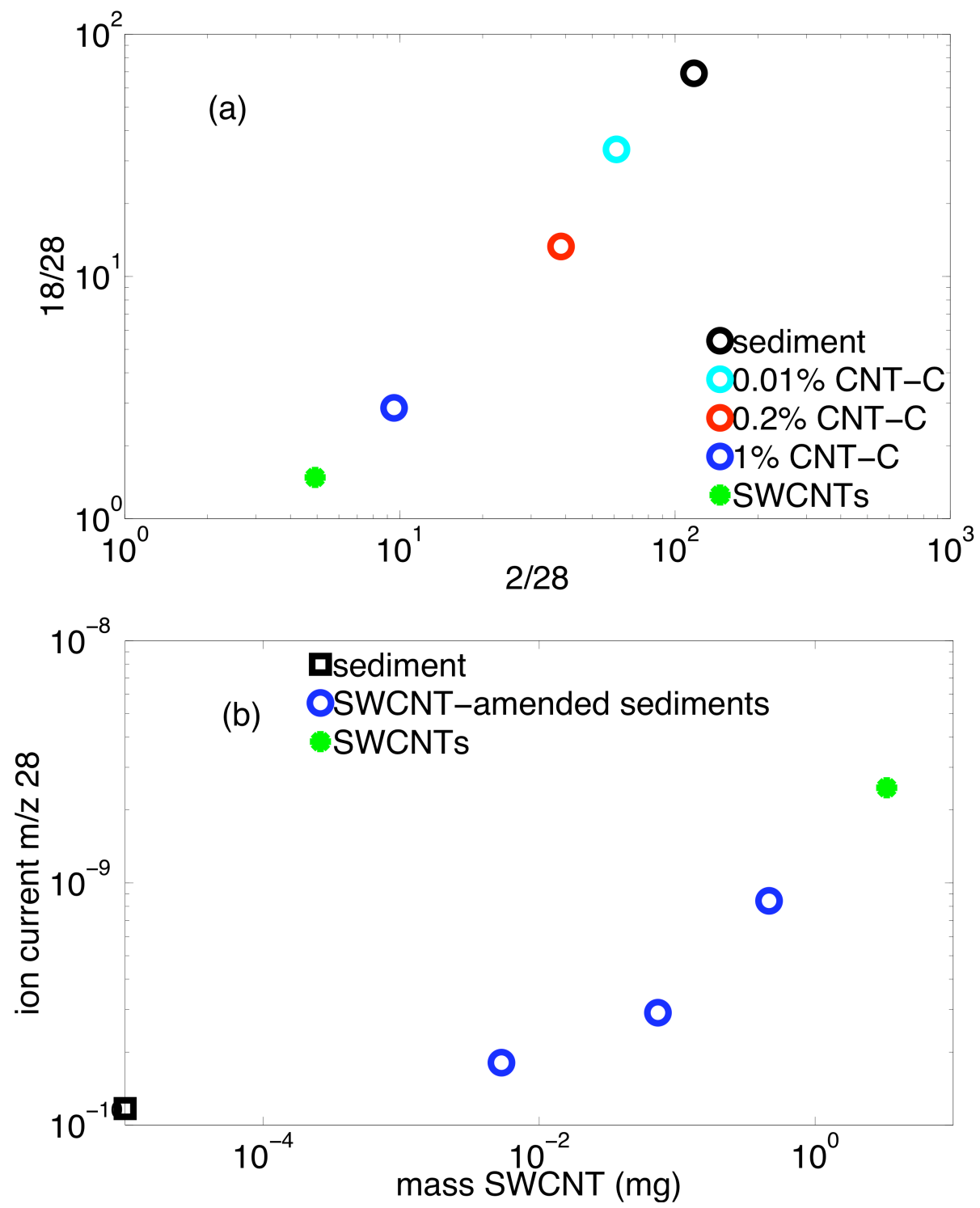

Figure 7. Diagnostic ion ratios observed in hydrogen-assisted thermal degradation of SWCNT-amended coastal sediments. Increasing amounts (weight percent indicated in figure legend) of SWCNTs were added to sediment collected from Dorchester Bay, MA. (a) When subjected to hydrogen-assisted thermal degradation, pure SWCNTs yielded very little mass fragment 18 (due to $\mathrm{H}_{2} \mathrm{O}$ ) and consumed mass fragment 2 (due to $\mathrm{H}_{2}$ ), and depletions in the 18/28 and 30/28 ion ratios were observed with increasing SWCNT content of the sediments. (b) The lower bound on the detection of SWCNTs was set by either co-degrading organic material in the sediments, which generated ethene and increased the baseline signal of ion current $\mathrm{m} / \mathrm{z} 28$, or an air leak (containing $\mathrm{N}_{2}$ with a mass of 28) in the TGA-MS (which was not designed to be airtight). 
The detection limit in the HATDs was set by either (1) co-degradation of sedimentary phases or (2) interference in the mass 28 signal due to an air leak in the TGA-MS, which was not designed to be airtight (Figure 8). The latter limitation could be overcome by a modified instrument design where the TGA furnace and associated flush lines were air tight and any leaks in the mass spectrometer were addressed. Very sensitive mass spectrometers (with detection limits in the picogram range) exist, and the use of such an instrument over this residual gas analyzer (RGA) MS (whose detection limit is on the order of micrograms) would automatically improve the sensitivity of the analysis. In addition, the use of a larger sample size may increase the signal due to ethene, driving it above the interfering $\mathrm{N}_{2}$ signal. Currently, the volume of the sample pan $(300 \mathrm{uL})$ in the TA Instruments Q50 TGA limits the deliverable sample mass (approximately 45-60 mg of sediment, roughly 3-10 mg of SWCNTs, depending on their density), as the balance is capable of measuring masses up to $200 \mathrm{mg}$. Modifications in the pan shape and size and/or the balance mechanism may allow for larger input sample sizes. However, based on a $\mathrm{pg} \mathrm{g}^{-1}$ sediment $\mathrm{SWCNT}$ content (estimated by Plata et al. ${ }^{26}$ ), $1,000 \mathrm{~kg}$ of sediment would be needed to overcome the $10 \mathrm{ug}$ sensitivity of the TGA-MS. Clearly, substantial improvements in the instrumentation (e.g., reduced air leaks and increased MS sensitivity) are needed, as balance modifications are unreasonable at present.

\section{Current applications of TGA-MS to detect SWCNTs in the environment. In} spite of the limited sensitivity of this method, it can successfully distinguish between natural and engineered nanoparticles (i.e., BC soot and SWCNTs). Until now, this has been a significant challenge and presented an impasse to the quantification of engineered nanoparticle release from industrial and research-scale manufacturing sites. For example, several groups ${ }^{25,30,31}$ have relied on light-scattering techniques to measure the release of nanoparticles during CNT production or post-fabrication handling. In all cases, 30-50 nm particles were detected, but it was unclear whether these were BC soot or CNTs. Occasionally, EM is used to distinguish the two materials, but this method is not quantitative and is expensive and labor-intensive. Using TGA-MS, the particles could be 
collected and subjected to HATD, where SWCNTs would be degraded and quantified, while BC soot would be preserved for subsequent quantification (e.g., by elemental analysis). Thus, thermal analysis provides a low-cost, high-throughput method for the isolation and quantification of carbon phases that are otherwise difficult to distinguish. Given the simplicity of the approach, it is reasonable to expect that all interested parties (i.e., industry, government, and academic researchers) perform the analysis where there is potential for SWCNT release. This would allow industries and regulatory agencies to put an upper bound on sources of SWCNTs to the environment (e.g., $100 \mathrm{ug}_{\mathrm{SWCNT}} \mathrm{g}^{-1}$ sample). If the detection limit is exceeded, measures can be taken to prevent further release and potential ecological damage. At this early stage of development, these efforts are critical to preserving human and environmental health, as well as the public's confidence in the growing nanomaterials industry.

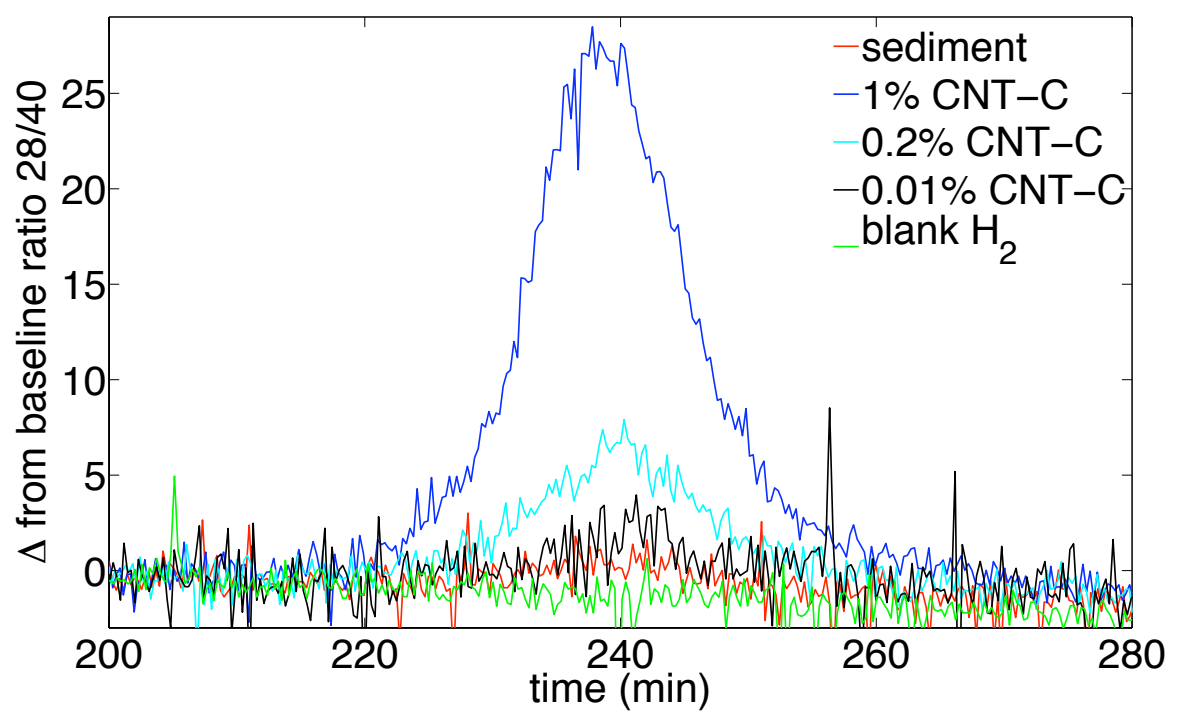

Figure 8. The sample-derived $\mathrm{C}_{2} \mathrm{H}_{4}$ signal can be visualized as a change in the ion current ratio 28/40 from its baseline, which is due to an air leak in the TGA-MS (which is not designed to be airtight). The $28 / 40$ current in air is constant, and the $\mathrm{C}_{2} \mathrm{H}_{4}$ signal due to SWCNTs decreases steadily with decreasing SWCNT content of SWCNT-amended sediments. The background signal of 28 (due to an air leak) in a blank run of pure $\mathrm{H}_{2}$ carrier gas is not substantially different than the signal generated from the degradation of SWCNT-free sediments. Thus, it may be possible to reduce the current detection limit by eliminating the air leak in the TGA-MS. 


\section{ACKNOWLEDGMENTS}

The authors thank the Martin Family Society of Fellows for Sustainability and Arunas and Pam Chesonis through MIT's Earth System Initiative for their support of this work. We also thank the Institute for Soldier Nanotechnologies at MIT, the Gschwend Lab, and John MacFarlane.

\section{REFERENCES}

[1] de Heer, W.A. Nanotubes and the pursuit of applications. MRS Bulletin April 2004, 281-285.

[2] Ball, P. Roll up for the revolution. Nature. 2001, 414, 142-144.

[3] Shi Kam, N.W.; O’Connell, M.; Widsdon, J.A.; Dai, H. Carbon nanotubes as multi-functional biological transporters and near-infrared agents for selective cancer cell destruction. PNAS. 2005, 102(33), 11600-11605.

[4] Thayer, A.M. Carbon nanotubes by the metric ton. Chemical \& Engineering News 2007, 85 (46), 29-35.

[5] Lam, C-W.; James, J.T.; McCluskey, R.; Hunter, R.L. Pulmonary toxicity of single-wall carbon nanotube in mice $7 \& 90$ days after intratracheal instillation. Toxicol. Sciences. 2004, 77, 126-134.

[6] Jia, G.; Wang, H.; Yan, L.; Wang, X.; Pei, R.; Yan, T.; Zhao, Y.; Guo, X. Cytotoxicity of carbon nanomaterials: single-wall nanotube, multi-wall nanotube, and fullerene. Environ. Sci. Technol. 2005, 39, 1378-1383.

[7] Poland, C.A.; Duffin, R.; Kinloch, I.; Maynard, A.; Wallace, W.A.H.; Seaton, A.; Stone, V.; Brown, S.; MacNee, W.; Donaldson, K. Carbon nanotubes introduced intot he abdominal cavity of mice show asbestoslike pathogenicity in a pilot study. Nature Nanotechnology 2008, 3, 423-428.

[8] Templeton, R.C.; Ferguson, P.L.; Washburn, K.M.; Scrivens, W.A.; and Chandler, G.T. Life-cycle effects of single-walled carbon nanotubes (CNTs) on an estuarine meiobenthic copepod. Environ. Sci. Technol. 2006, 40(23), 73877393. 
[9] Plata, D.L., Gschwend, P.M., Reddy, C.M. Industrially synthesized singlewalled carbon nanotubes: compositional data for users, environmental risk assessments, and source apportionment. Nanotechnology 2008, 19, 185706.

[10] Girifalco, L.A.; Hodak, M.; Lee, R.S. Carbon nanotubes, buckyballs, ropes, and a universal graphitic potential. Phys. Rev. B 2000, 62, 12104-13110.

[11] Goldberg, E.D. Black carbon in the environment: Properties and Distribution. John Wiley \& Sons, New York, NY. 1985.

[12] Hammes, K.; et 32 co-authors. Comparison of quantification methods to measure fire-derived (black/elemental) carbon in soils and sediments using reference materials from soil, water, sediment and the atmosphere. Global Biogeochemical Cycles 2007, 21, GB3016.

[13] Gustafsson, Ö., Haghseta, F.; Chan, C.; MacFarlane, J.; Gschwend, P.M. Quantification of the dilute sedimentary soot phase: implications for PAH speciation and bioavailability. Environ. Sci. Technol. 1997, 31, 203-209.

[14] Harutyunyan, A.R.; Pradhan, B.K.1 Chang, J.; Chen, G.; Eklund, P.C. Purification of single-wall carbon nanotubes by selective microwave heating of catalyst particles. J. Phys. Chem. B 2002, 106, 8671-8675.

[15] Accardi-dey, A.M.; Gschwend, P.M. Assessing the combined roles of natural organic matter and black carbon as sorbents in sediments. Environ. Sci. Technol. 2002, 36, 21-29.

[16] Flores-Cervantes, D.X. Black carbon in the Gulf of Maine: New insights into inputs and cycling of combustion-derived organic carbon. MIT Thesis. 2008.

[17] López-Fonseca, R.; Landa, I.; Elizundia, U.; Gutiérrez-Ortiz, M.A., GonzálezVelasco, J.R. Thermokinetic modeling for the combustion of carbonaceous particulate matter. Combustion and Flame 2006, 144, 398-406.

[18] Chiang, I.W.; Brinson, B.E.; Smalley, R.E.; Margrave, J.L.; Hauge, R.E. Purification and characterization of single-wall carbon nanotubes. J. Phys. Chem. $B$ 2001, 105, 1157-1161.

[19] Herrera, J.E.; Resasco, D.E. In situ TPO/Raman to characterize single-walled carbon nanotubes. Chem. Phys. Lett. 2003, 376, 302-309.

[20] Merritt, D.A.; Hayes, J.M.; Des Marais, D.J. Carbon isotopic analysis of atmospheric methane by isotope-ratio-monitoring gas chromatography-mass spectrometry. J. Geophys. Res. 1995, 100 (D1), 1317-1326. 
[21] Bridgewater, J.D.; Lim, J.; Vachet, R.W. Transistion metal-peptide binding studied by metal-catalyzed oxidation reactions and mass spectrometry. Anal. Chem. 2006, 78 (7), 2432-2438.

[22] Alexander, B.; Park, R.J.; Jacob, D.J.; Gong, S. Transistion metal-catalyzed oxidation of atmospheric sulfur: Global implications for the sulfur budget. $J$. Geophys. Res. 2009, 114, D02309 (13 pp).

[23] Schmidt, M.W.I.; Noack, A.G. Black carbon in soils and sediments: Analysis, distribution, implications, and current challenges. Global Biogeochemical Cycles 2000, 14 (3), 777-793.

[24] Preston, C.M.; Schmidt, M.W.I. Black (pyrogenic) carbon: a synthesis of current knowledge and uncertainties with special consideration of boreal regions.

Biogeosciences 2006, 3, 397-420.

[25] Maynard, A.D.; Baron, P.A.; Foley, M.; Shvedova, A.A., Kisin, E.R.; Castranova, V. Exposure to carbon nanotube material: Aerosol release during the handling of unrefined single-walled carbon nanotube material. J. Toxicol. Environ. Health A 2004, 67, 87-107.

[26] Plata, D.L. Carbon nanotube synthesis and detection: Limiting the environmental impact of novel technologies. Chapter 1. MIT Thesis 2009.

[27] Reddy, C.M., Pearson, A., Xu, L., McNichol, A.P., Benner, B.A., Wise, S.A., Klouda, G.A., Currie, L.A.; Eglinton, T.I. Radiocarbon as a tool to apportion the sources of polycyclic aromatic hydrocarbons and black carbon in environmental samples. Environ. Sci. Technol. 2002, 36, 1774-1782.

[28] SouthWest Nano Technologies, Product Data Sheet, http://www.swnano.com/tech/sg.php. Accessed 15 Jan 2009.

[29] Petersen, E.J.; Akkanen, J.; Kukkonen, J.V.; Weber, W.J. Biological uptake and depuration of carbon nanotubes by Daphnia magna. Environ. Sci. Technol. 2009. ASAP Articles DOI: 10.1021/es8029363

[30] Yeganeh, B.; Kull, C.M.; Hull, M.S.; Marr, L.C. Characterization of airborne particles during production of carbonaceous nanomaterials. Environ. Sci. Technol. 2008, 42 (12), 4600-4606.

[31] Bello, D.; Hart, A.J.; Ahn, K.; Hallock, M.; Yamamoto, N.l Garcia, E.J.; Wardle, B.L.; Ellenbecker, M.J. Particle exposure levels during growth and subsequent handling aligned carbon nanotube films. Carbon 2008, 46, 974-981. 


\section{SUPPORTING INFORMATION}

\section{Potential oxidizing power of SWCNT metal catalysts}

Table S1. Reduction potentials for various metals present in SWCNT powders.

\begin{tabular}{c|c} 
Half-reaction & Reduction potential $\left(\mathbf{E}^{\mathbf{0}}(\mathbf{V})\right)$ \\
\hline $\mathrm{Y}(\mathrm{III})+3 e^{-}=\mathrm{Y}(0)(s)$ & -2.37 \\
$\mathrm{Fe}(\mathrm{II})+2 e^{-}=\mathrm{Fe}(0)(s)$ & -0.44 \\
$\mathrm{Co}(\mathrm{II})+2 e^{-}=\mathrm{Co}(0)(s)$ & -0.28 \\
$\mathrm{Ni}(\mathrm{II})+2 e^{-}=\mathrm{Ni}(0)(s)$ & -0.25 \\
$\mathrm{Me}(\mathrm{III})+3 e^{-}=\mathrm{Fe}(0)(s)$ & -0.15 \\
$\mathrm{MoO}_{2}(s)+4 \mathrm{H}^{+}+4 e^{-}=\mathrm{Mo}(0)(s)+2 \mathrm{H}_{2} \mathrm{O}$ & -0.04
\end{tabular}

Comparing two-electron transfer reactions, $\mathrm{Ni}(\mathrm{II})$ is a superior oxidant compared to both $\mathrm{Co}(\mathrm{II})$ and $\mathrm{Fe}(\mathrm{II})$. Thus, from a very simplified perspective (ignoring, for example, metal abundance and oxygen physisorption processes), one might expect that SWCNTs that contain $\mathrm{Ni}(\mathrm{II})$ will have the lower oxidation temperatures than SWCNTs that contain $\mathrm{Co}(\mathrm{II})$ and $\mathrm{Fe}(\mathrm{II})$. Ni-containing CNTs (Ni and Y) did have lower oxidation temperatures $\left(\mathrm{T}_{\max , \text { oxidation }}=413-428^{\circ} \mathrm{C}\right)$ than SWCNTs that contained Fe, Co, and Mo $\left(\mathrm{T}_{\text {max,oxidation }}=\right.$ $\left.510-540^{\circ} \mathrm{C}\right)$ or Fe and Mo alone $\left(\mathrm{T}_{\max , \text { oxidation }}=505^{\circ} \mathrm{C}\right)($ Figure 2$)$. However, Fe(III) and Mo(IV) are more efficient oxidants than Ni(II), and so SWCNTs that contain Mo(IV) and $\mathrm{Fe}(\mathrm{III})$ might be expected to have lower oxidation temperatures than were observed. Considering the relatively high thermal stabilities of the Fe/Mo- and Fe/Mo/Cocontaining SWCNTs as compared to the Ni-containing SWCNTs (and ignoring the metal catalysts' abundances, influence on SWCNT structure, and ability to physisorb oxygen), one might infer that the Fe and Mo present in the Fe/Mo- and Fe/Mo/Co-containing SWCNTs is not Fe(III) or Mo(IV). The SWCNT sample that contained purely Fe had a low oxidation temperature, consistent with the presence of Fe(III) (but note that the mass of Fe in this sample was very high). Y(III) is expected to be a poor oxidant, but all Ni/Y samples had low oxidation temperatures, perhaps suggesting that $\mathrm{Ni}$ (II) was principally responsible for the oxidation of Ni-containing SWCNTs. 


\section{Mass spectra of evolved gases from the thermal treatment of standard materials}

Data processing. Mass spectral data was normalized and/or background subtracted, depending on particular ion interferences that arise from choice of carrier gas.

Generating mass spectra for test materials:

For each test material, the spectrum of ions, $i$, at the peak oxidation temperature ( $\mathrm{Y}_{i, \text { maximum,sample }}$ ) was corrected for the background signal and contributions from unresolved phases of the test material. We chose the mass spectrum at an oxidation minimum (trough or valley) preceding or following the peak of interest to represent the contributions from background and unresolved phases ( $\left.\mathrm{Y}_{\text {i,minimum, background }}\right)$. To account for signal drift during the thermal program, the minimum signal was normalized using ion current 40 (for the pyrolyses) or ion current 28 (for the oxidations) (represented as ion $\mathrm{X}$ in Eqn $\mathrm{S} 1)$.

$$
Y_{i, \text { sample, }, \text { corrected }}=Y_{i, \text { maximum, sample }}-Y_{i, \text { minimum,background }}\left(\frac{X_{\text {maximum, sample }}}{X_{\text {minimum,background }}}\right)
$$


Figure S1. Mass spectra of evolved gases from the temperature programmed oxidation (TPO) of (a) SWeNT Purified SWCNTs, (b) diesel particulate matter (NIST SRM 1650), (c) methylcellulose, and (d) bovine serum albumin. All spectra were background subtracted and are shown at the maximum oxidation temperature of the respective test material.
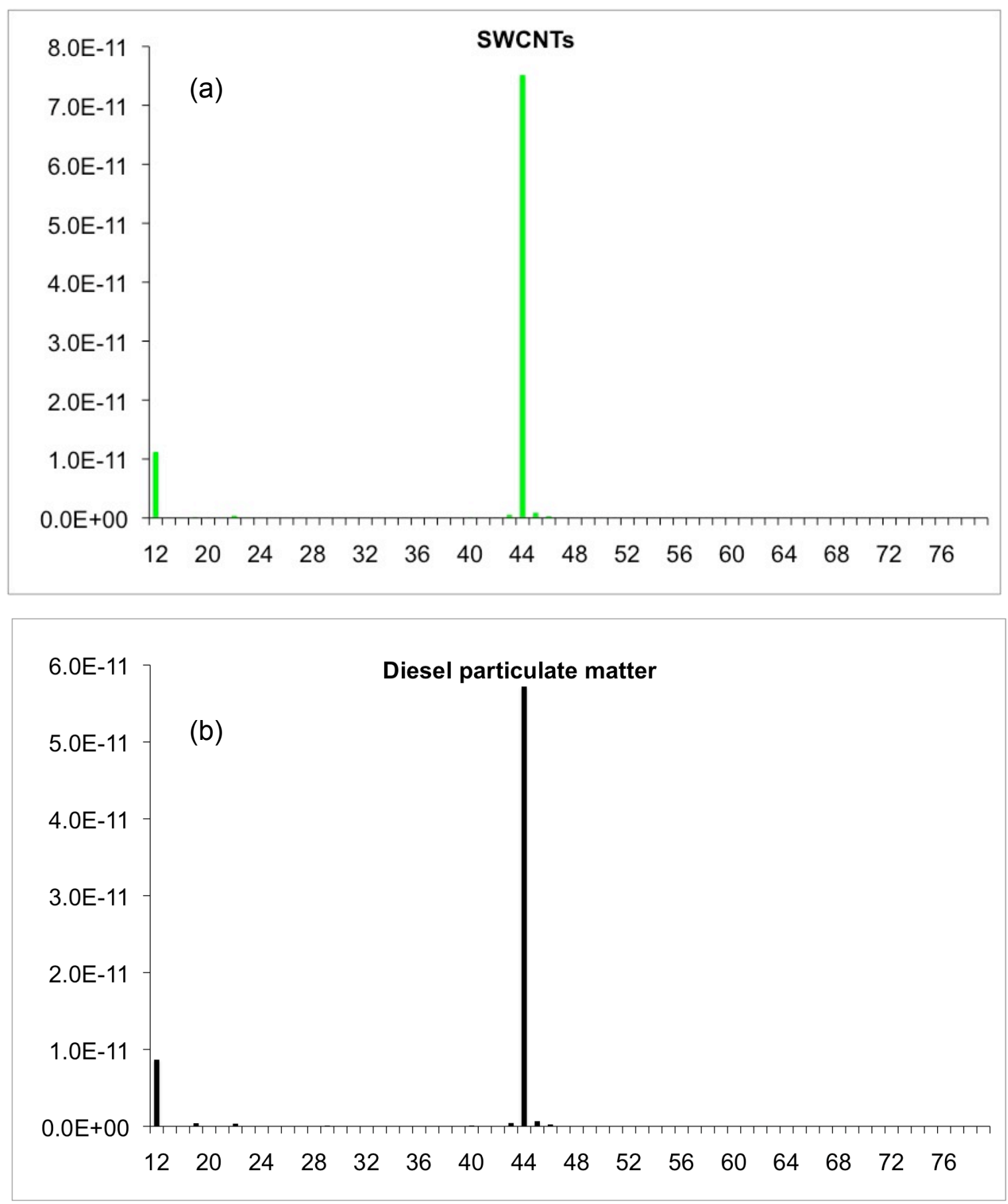

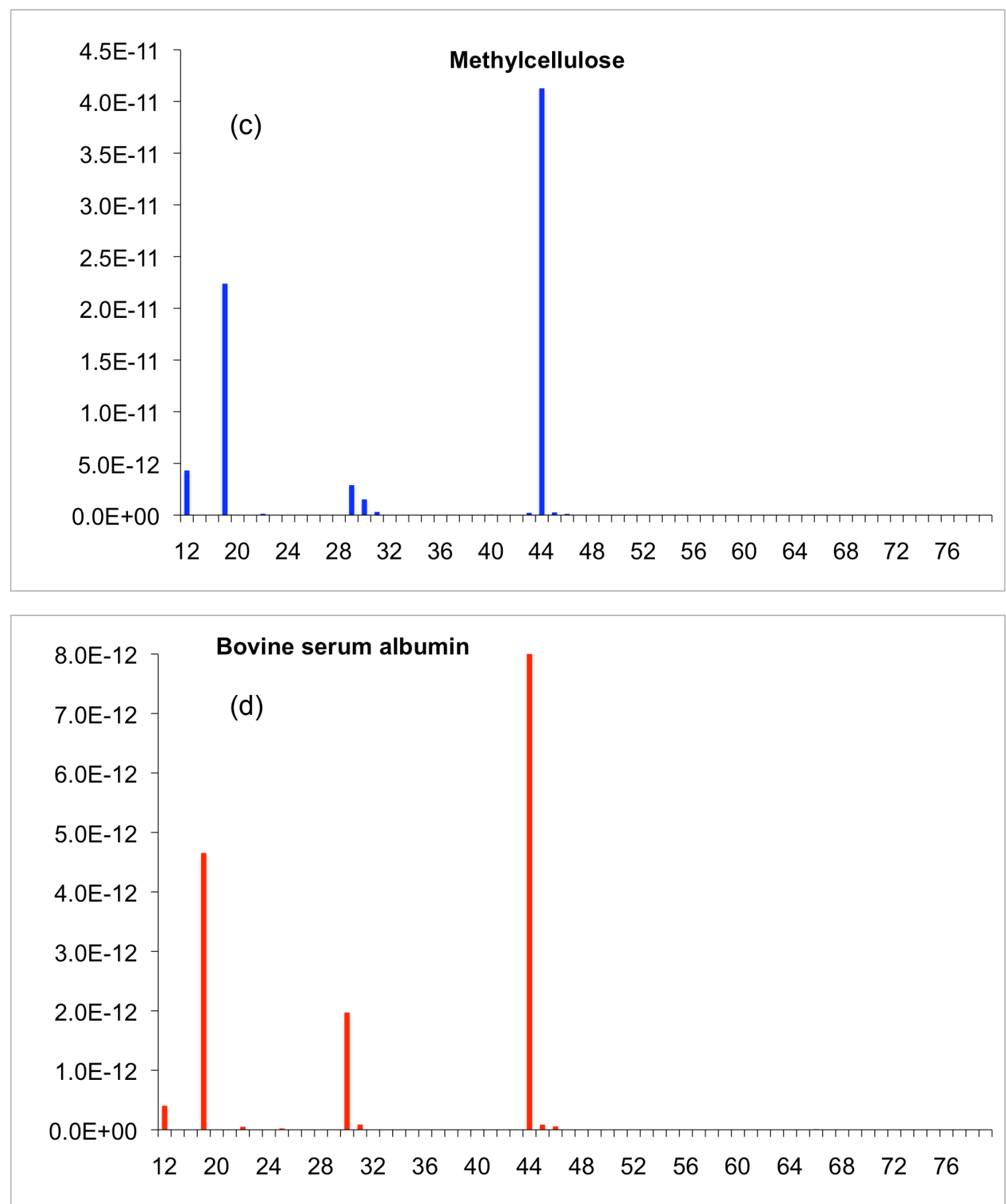
MWCNT and $\mathrm{C}_{60}$ thermal stability during TPO and HATD

Figure S2. Differentiated thermogram of MWCNTs and $\mathrm{C}_{60}$ during TPO and HATD.

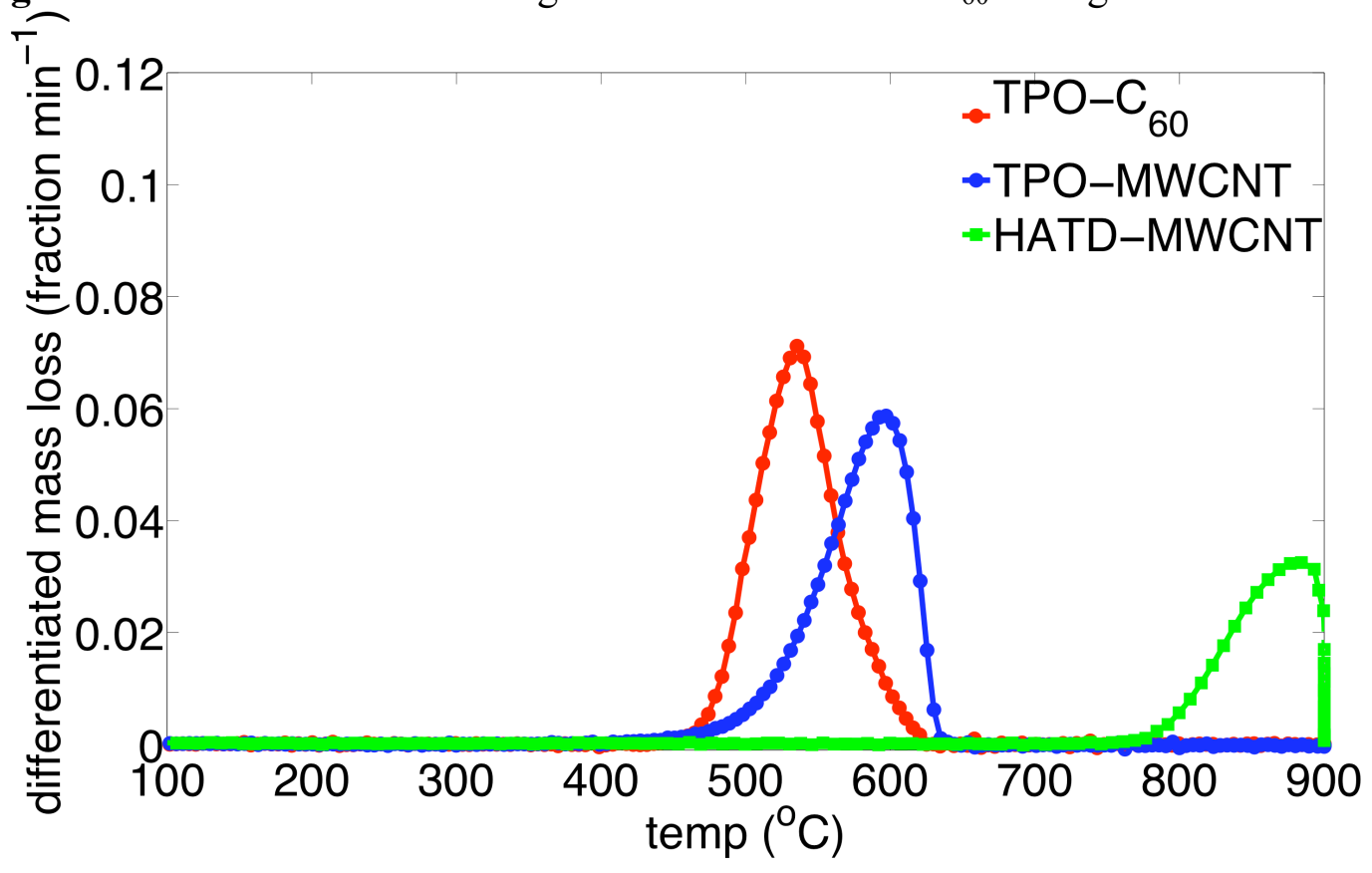


Attempts to reduce interferences in SWCNT oxidation temperature using different thermal programs.

Figure S4. Sediment and SWCNT-amended sediment were subjected to the following temperature program in dry air: Ballistic heat to $80 \mathrm{oC}, 15 \mathrm{~min}$ hold, ballistic heat to $200^{\circ} \mathrm{C}, 15 \mathrm{~min}$ hold, ballistic heat to $300^{\circ} \mathrm{C}, 30 \mathrm{~min}$ hold, ballistic heat to $425^{\circ} \mathrm{C}, 30 \mathrm{~min}$ hold, ballistic heat to $600^{\circ} \mathrm{C}, 300 \mathrm{~min}$ hold, ballistic heat to $900^{\circ} \mathrm{C}, 30 \mathrm{~min}$ hold. The differentiated mass loss plot (a) and ion current for mass $44\left(\mathrm{CO}_{2}\right)(\mathrm{b})$ are shown.
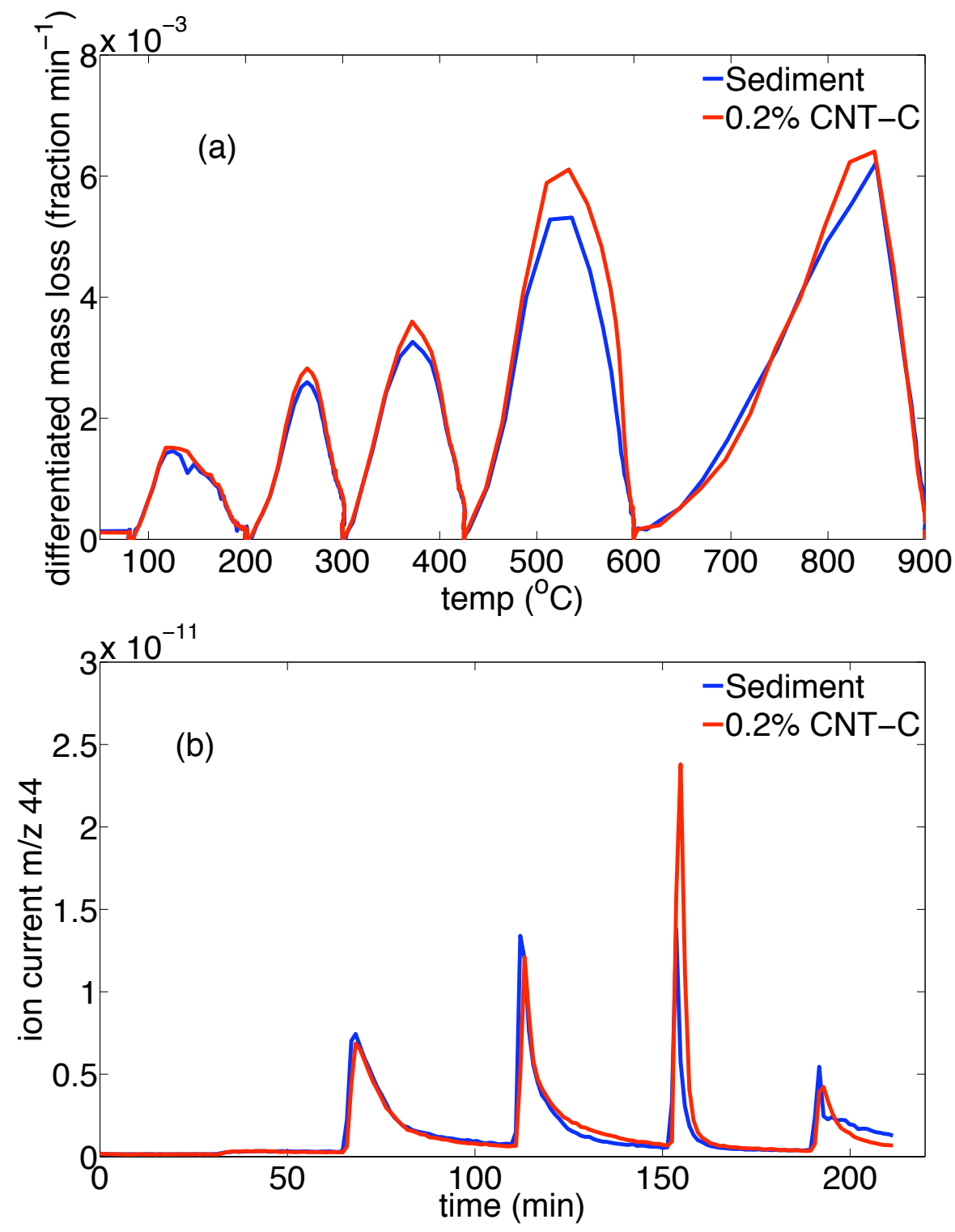
Figure S5. SWCNTs (SWP) and diesel particulate matter (NIST 1650) were subjected to the following temperature program in dry air: Ballistic heat to $80 \mathrm{oC}, 15 \mathrm{~min}$ hold, ballistic heat to $200^{\circ} \mathrm{C}, 15 \mathrm{~min}$ hold, ballistic heat to $300^{\circ} \mathrm{C}, 30 \mathrm{~min}$ hold, ballistic heat to $425^{\circ} \mathrm{C}, 30 \mathrm{~min}$ hold, ballistic heat to $600^{\circ} \mathrm{C}, 300 \mathrm{~min}$ hold, ballistic heat to $900^{\circ} \mathrm{C}, 30 \mathrm{~min}$ hold. The differentiated mass loss plot (a) and ion current for mass $44\left(\mathrm{CO}_{2}\right)(\mathrm{b})$ are shown.
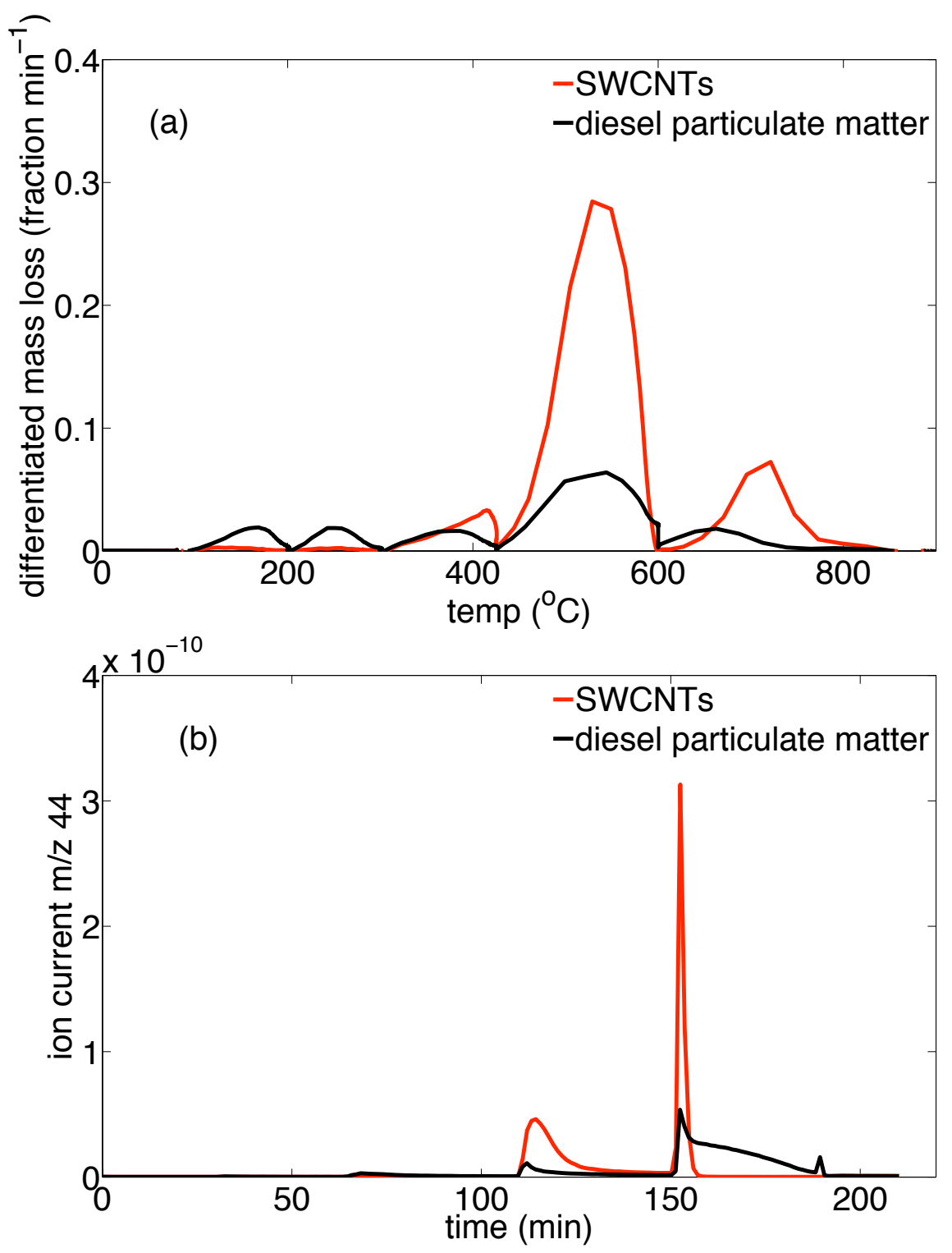
Figure S6. SWCNTs (SWP) and diesel particulate matter (NIST 1650) were subjected to the following temperature program in dry air: Ballistic heat to $80 \mathrm{oC}, 15 \mathrm{~min}$ hold, ballistic heat to $375^{\circ} \mathrm{C}, 30 \mathrm{~min}$ hold, ballistic heat to $500^{\circ} \mathrm{C}, 60 \mathrm{~min}$ hold, ballistic heat to $900^{\circ} \mathrm{C}, 15 \mathrm{~min}$ hold. The differentiated mass loss plot (a) and ion current for mass 44 $\left(\mathrm{CO}_{2}\right)(\mathrm{b})$ are shown.
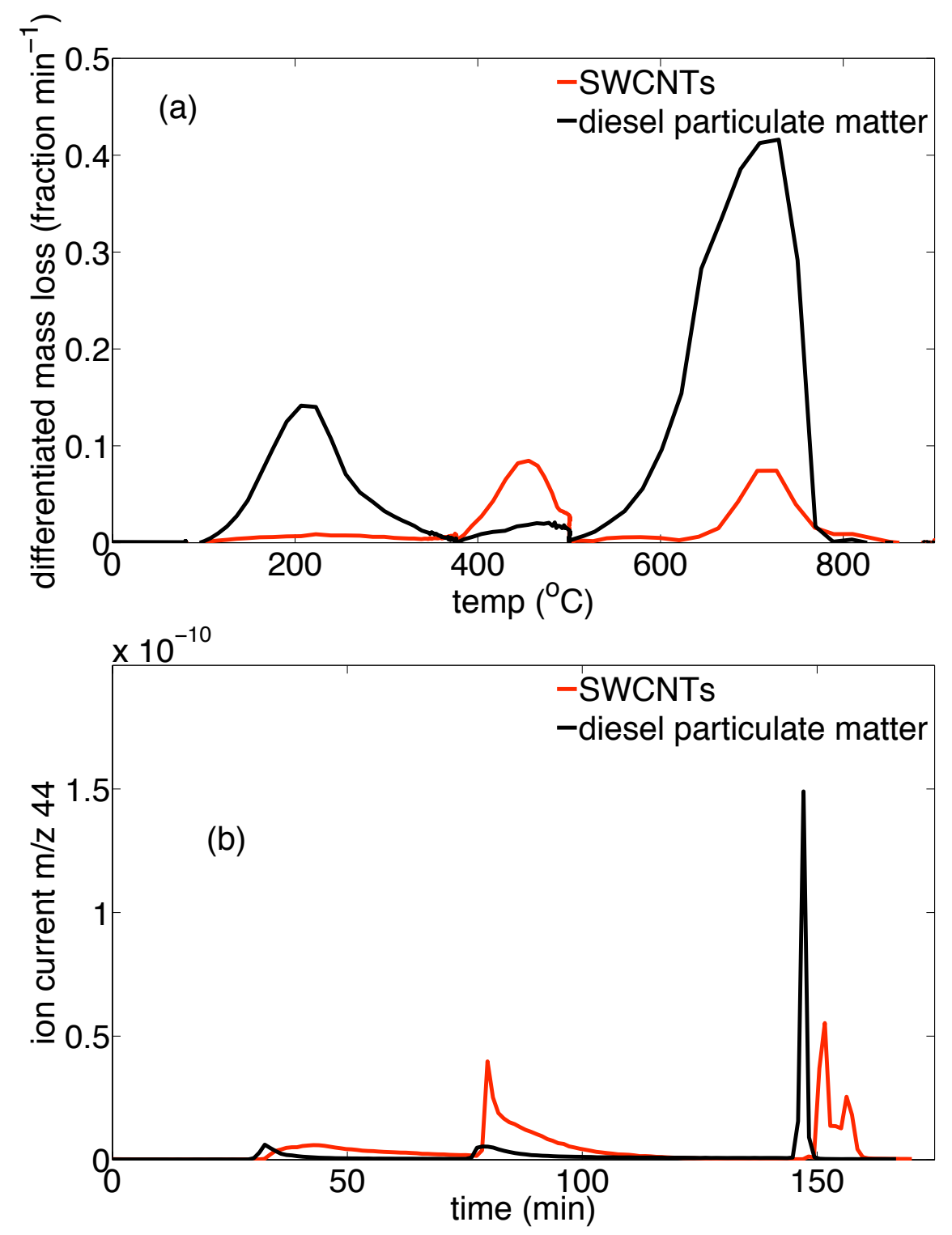
Figure S7. Sediment and SWCNT-amended sediment were subjected to the following temperature program in dry air: Ballistic heat to $80 \mathrm{oC}, 15 \mathrm{~min}$ hold, ballistic heat to $375^{\circ} \mathrm{C}, 90 \mathrm{~min}$ hold (not this step is 60 minutes longer than in Figure S6 (relatively pure materials), to allow sufficient time for sedimentary phases to oxidize before the next ramp step), ballistic heat to $500^{\circ} \mathrm{C}, 60 \mathrm{~min}$ hold, ballistic heat to $900^{\circ} \mathrm{C}, 15 \mathrm{~min}$ hold. The differentiated mass loss plot (a) and ion current for mass $44\left(\mathrm{CO}_{2}\right)(\mathrm{b})$ are shown.
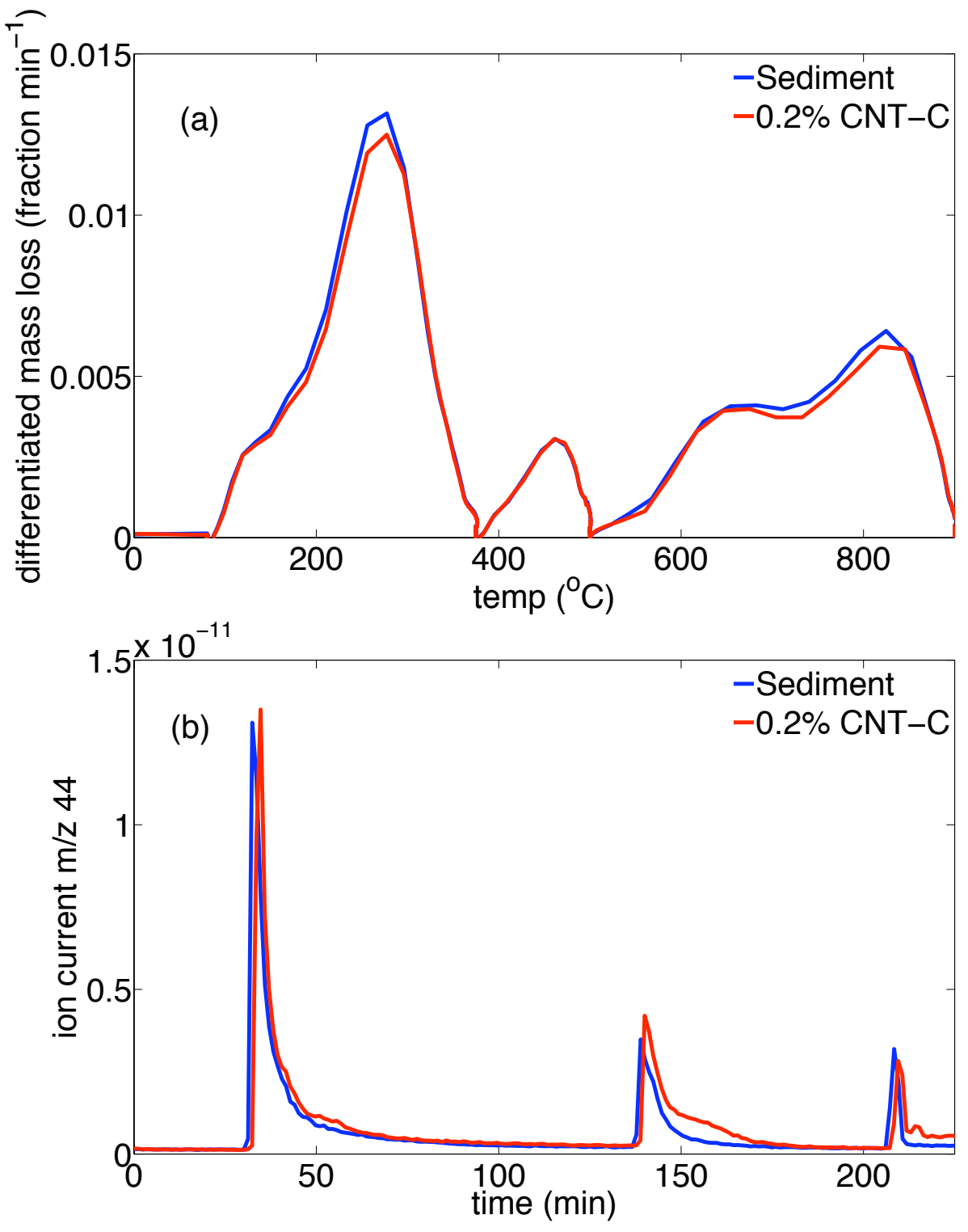
Figure S8. Sediment and SWCNT-amended sediment were subjected to the following

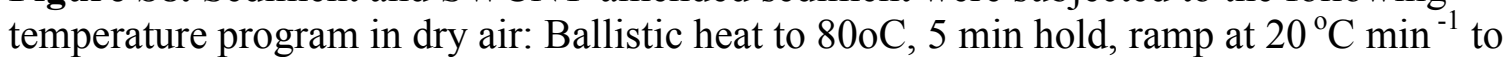
$250^{\circ} \mathrm{C}, 5 \mathrm{~min}$ hold, ramp at $20^{\circ} \mathrm{C} \mathrm{min}^{-1}$ to $375^{\circ} \mathrm{C}, 60 \mathrm{~min}$ hold, ballistic heat to $500^{\circ} \mathrm{C}, 15$ min hold, ballistic heat to $600^{\circ} \mathrm{C}, 15 \mathrm{~min}$ hold, ballistic heat to $900^{\circ} \mathrm{C}, 5 \mathrm{~min}$ hold. The differentiated mass loss plot (a) and ion current for mass $44\left(\mathrm{CO}_{2}\right)$ (b) are shown.
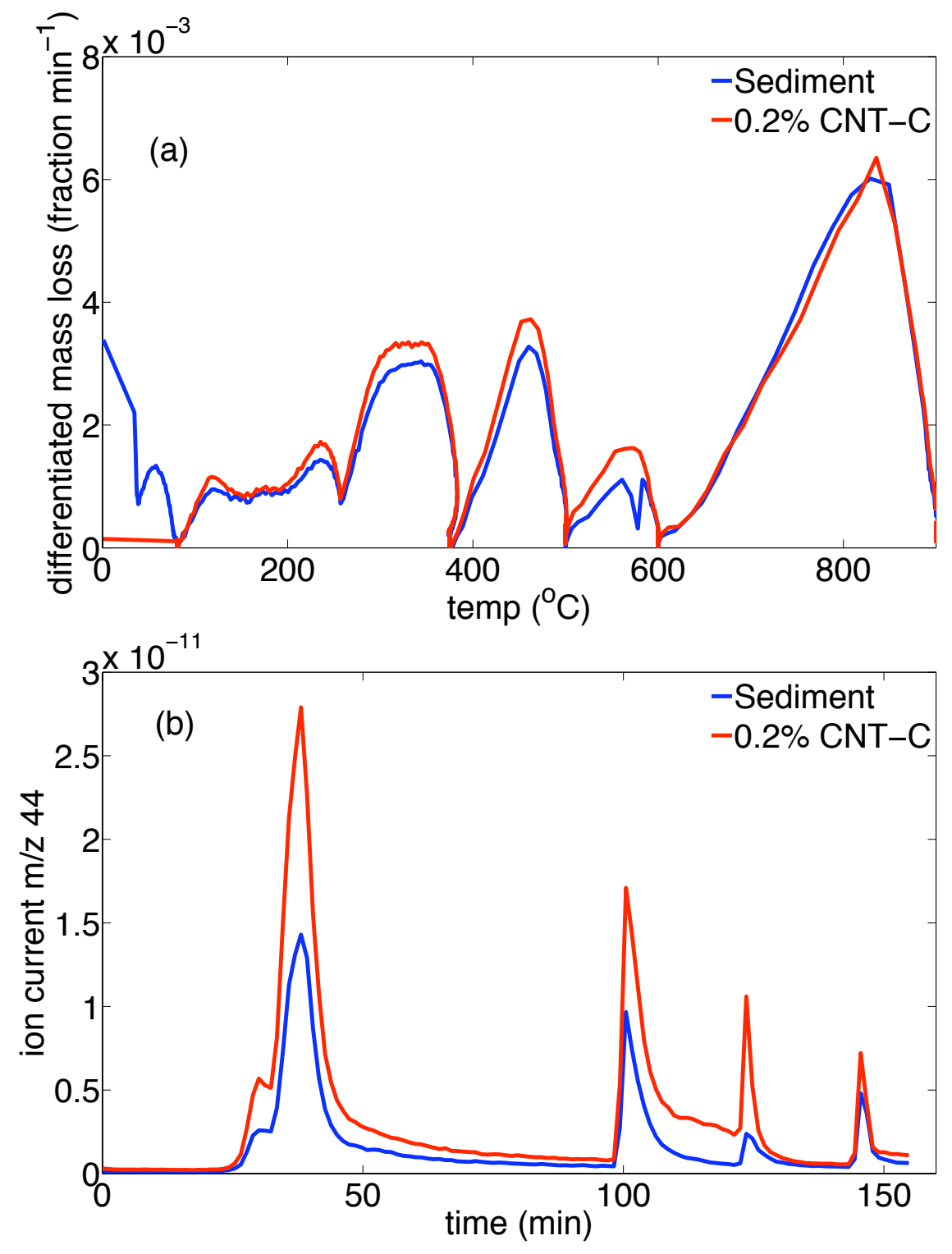


\section{Stable carbon isotopic content as a tool to quantify SWCNTs in complex mixtures}

Figure S9. Stable carbon isotopic content of Dorchester Bay sediment and SWCNTamended Dorchester Bay sediment after varying degrees of thermal treatment. Isotopic content is reported relative to a standard, Vienna Pee Dee Belemnite (VPDB), and the "delta notation" is defined in Eqn S2. Experimental details are described in the text of the Supporting Information.

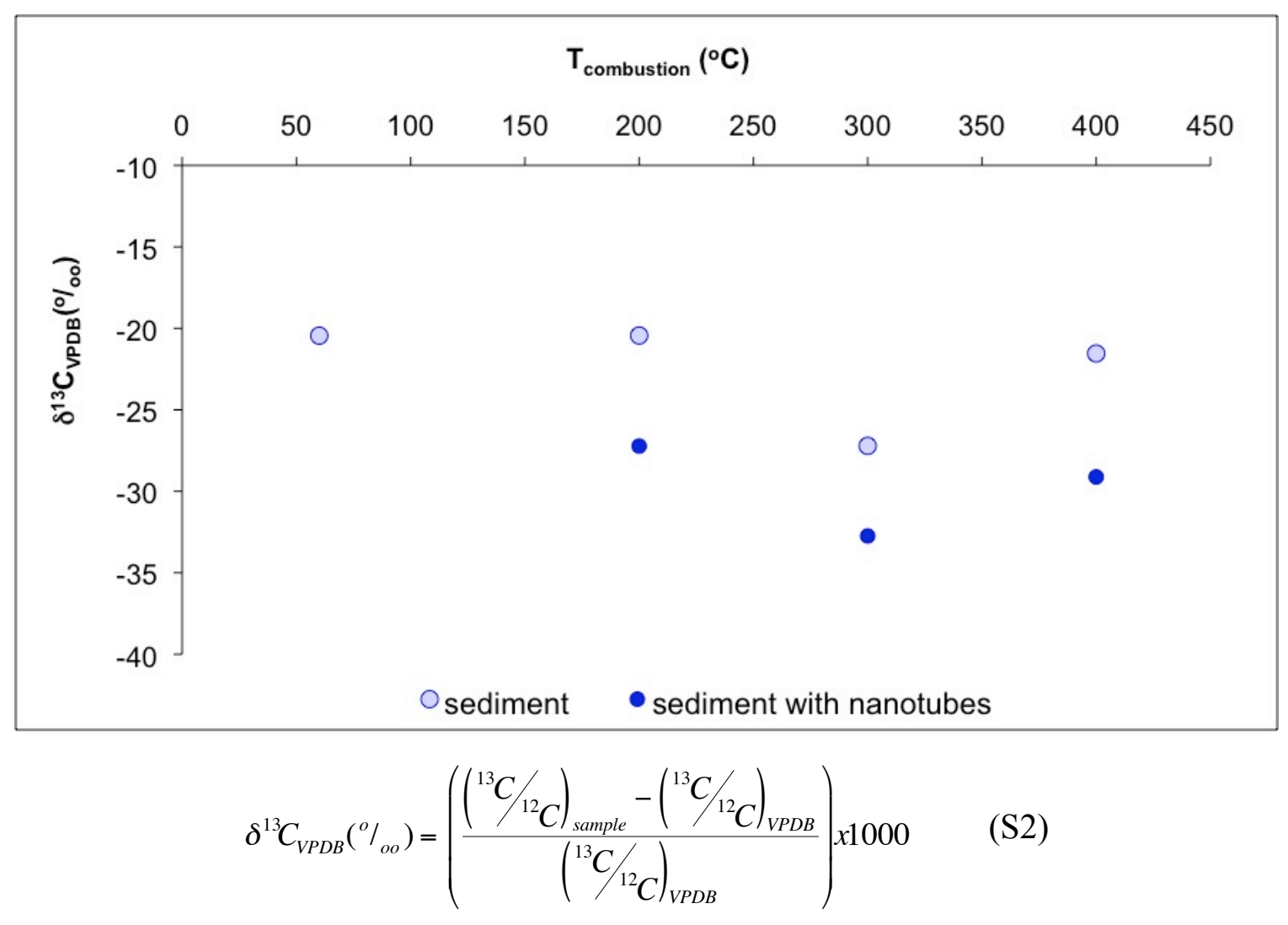

In this series of experiments, sediments from Dorchester Bay, MA were amended with HiPCO ${ }^{\mathrm{TM}}$ SWCNTs produced by Carbon Nanotech, Inc. (CNI, now Unidym) at 0.2 $\%$ dry weight. These SWCNTs are depleted in ${ }^{13} \mathrm{C}$ relative to the sediments $\left({ }^{13} \mathrm{C}_{\mathrm{SWP}}=-\right.$ $52.0 \%$ ). Native and amended sediments (5 mg samples) were well distributed in the pan to provide adequate oxygen access, acidified with $\mathrm{H}_{2} \mathrm{SO}_{3} / \mathrm{H}_{2} 0(50: 50 \mathrm{v} / \mathrm{v})$, and dried overnight at $60^{\circ} \mathrm{C}$. Each sample was then oxidized at the test temperature (e.g., 200, 300, or $400^{\circ} \mathrm{C}$ ) in TOC air (very low carbon content for total organic carbon (TOC) analyzers) for $1.5 \mathrm{hrs}$ in pre-combusted silver pans in a furnace. Following oxidation, samples were 
enclosed in silver pans and sealed into pre-combusted quartz tubes that contained roughly $2 \mathrm{~g}$ of $\mathrm{CuO}$. (Note that the silver pan is critical for the removal of residual sulfur from the acidification step. Failure to remove sulfur will result the formation of interfering sulfur oxides). After a 5 -hr oxidation at $800^{\circ} \mathrm{C}$, quartz tubes were cracked on a vacuum line, and resultant gases were purified cryogenically, quantified, and analyzed for stable carbon isotopic content following McNichol et al. ${ }^{1}$

The isotopic depletion due to the CNI SWCNTs was easily detected at the relatively high SWCNT proportion of $0.2 \%$ (about a factor of two higher than BC levels in most marine sediments) (Figure S3). The isotopic content of the sediment changed as more labile marine organic material $\left({ }^{13} \mathrm{C}_{\text {sediment }}=-20.4 \%\right.$ was removed and more recalcitrant, isotopically depleted material remained. The increase in from $\delta^{13} \mathrm{C}_{\text {sediment }} 300$ to $400^{\circ} \mathrm{C}$ was attributed to the preferential oxidation of the light isotope, which would make the residual solid carbon enriched with respect to ${ }^{13} \mathrm{C}$.

Figure S10. Calculated SWCNT content of the SWCNT-amended sediment following thermal oxidation.

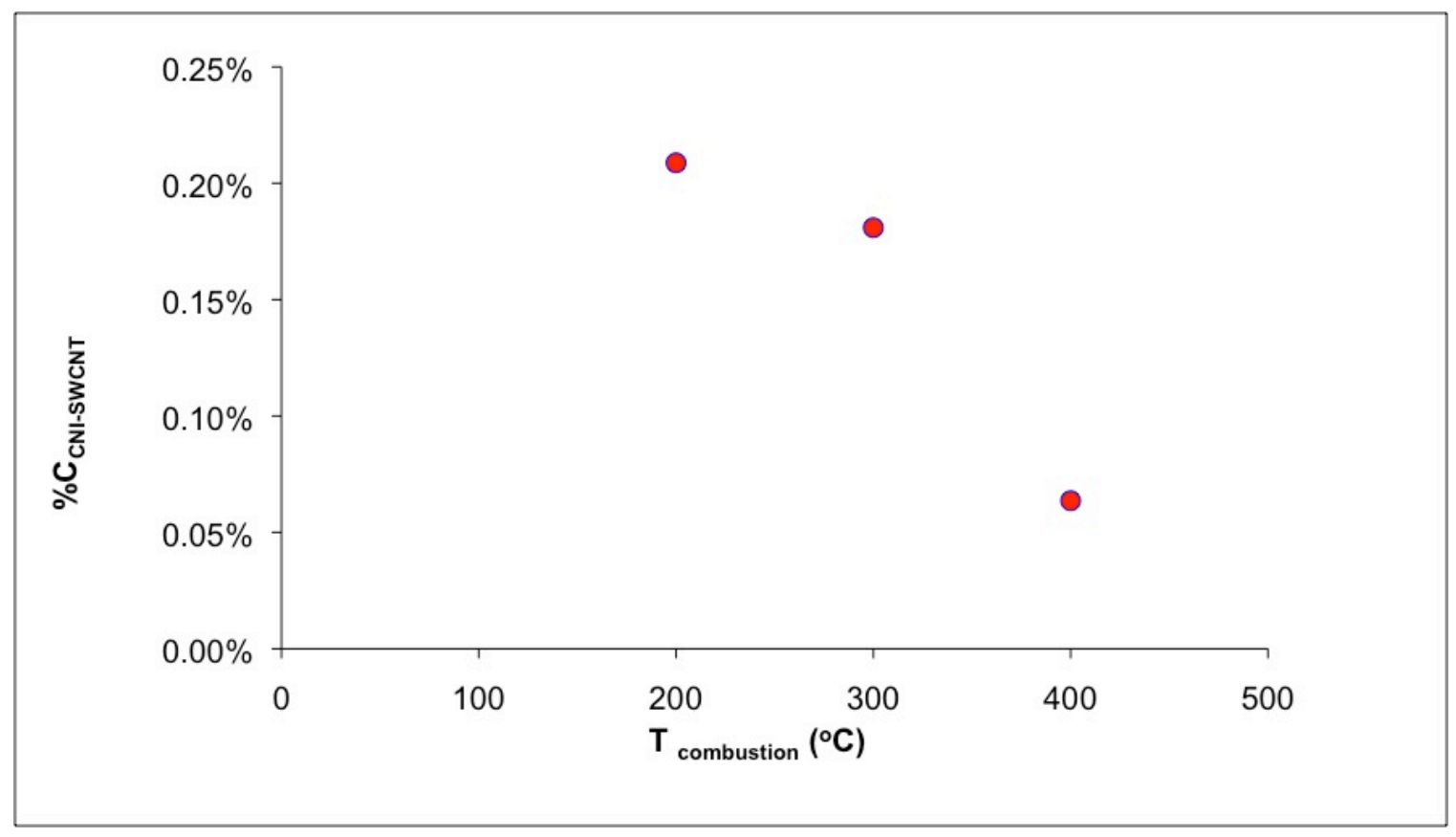


Using the isotopic content of the native and un-amended sediments, we calculated the proportion of SWCNTs remaining at each step of the thermal treatment (Eqn S3, where $f$ is the mass fraction of each respective material). The CNI SWCNTs began oxidize between 200 and $300^{\circ} \mathrm{C}$ and were largely degraded by $375^{\circ} \mathrm{C}$. This suggests that $\mathrm{CNI}$ SWCNTs will not be quantified as $\mathrm{BC}$ by traditional methods that define $\mathrm{BC}$ as material that survives a $24-\mathrm{hr}$ oxidation at $375^{\circ} \mathrm{C}$ in air (CTO-375 (Gustafsson \& Gschwend)). A similar approach could be used on gases evolved from thermal treatments by TGA, but assumptions would have to be made about the initial, SWCNT-free isotopic content of the sample matrix (i.e., $\delta^{13} \mathrm{C}_{\text {sediment }}$ in marine sediments, soil, aqueous filtrate, or aerosol concentrate). Additionally, this approach would not offer substantial improvements in the current detection limit of TGA-MS technique (see manuscript text for discussion).

$$
\delta^{13} C_{\text {sediment }+S W C N T}=f_{\text {sediment }} \delta^{13} C_{\text {sediment }}+f_{S W C N T} \delta^{13} C_{S W C N T}
$$




\section{Mass fragments generated during the hydrogen-assisted thermal decomposition of SWCNTs and other test materials.}

Figure S11. Mass spectra of evolved gases from the hydrogen-assisted thermal decomposition (HATD) of (a) SWeNT Purified SWCNTs, (b) Nanocyl MWCNTs, (c) methylcellulose, and (d) bovine serum albumin. No spectrum is shown for diesel particulate matter, as it did not degrade substantially during thermal treatment with pure $\mathrm{H}_{2}$. All spectra were background subtracted and are shown at the maximum degradation temperature of the respective test material.
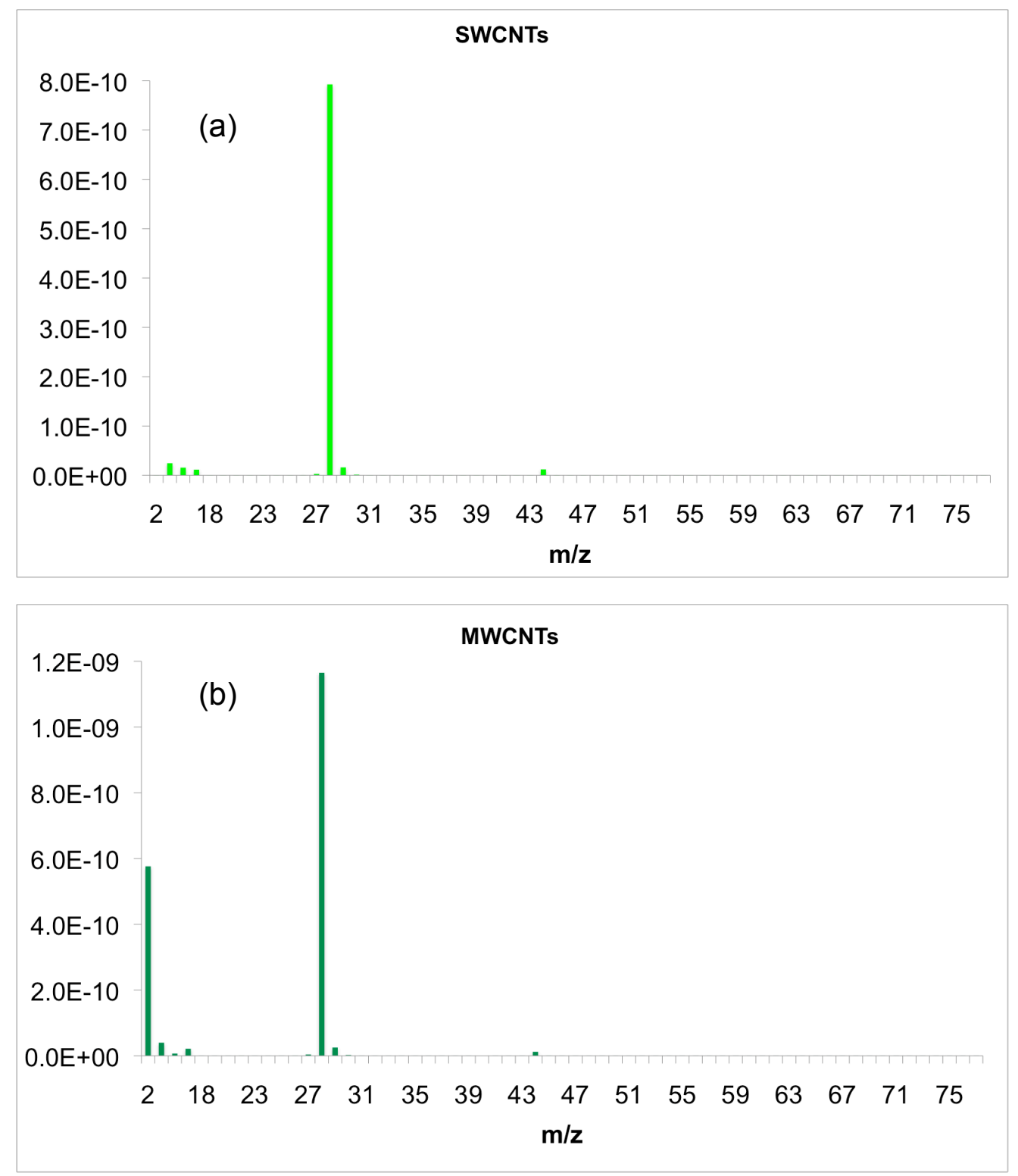

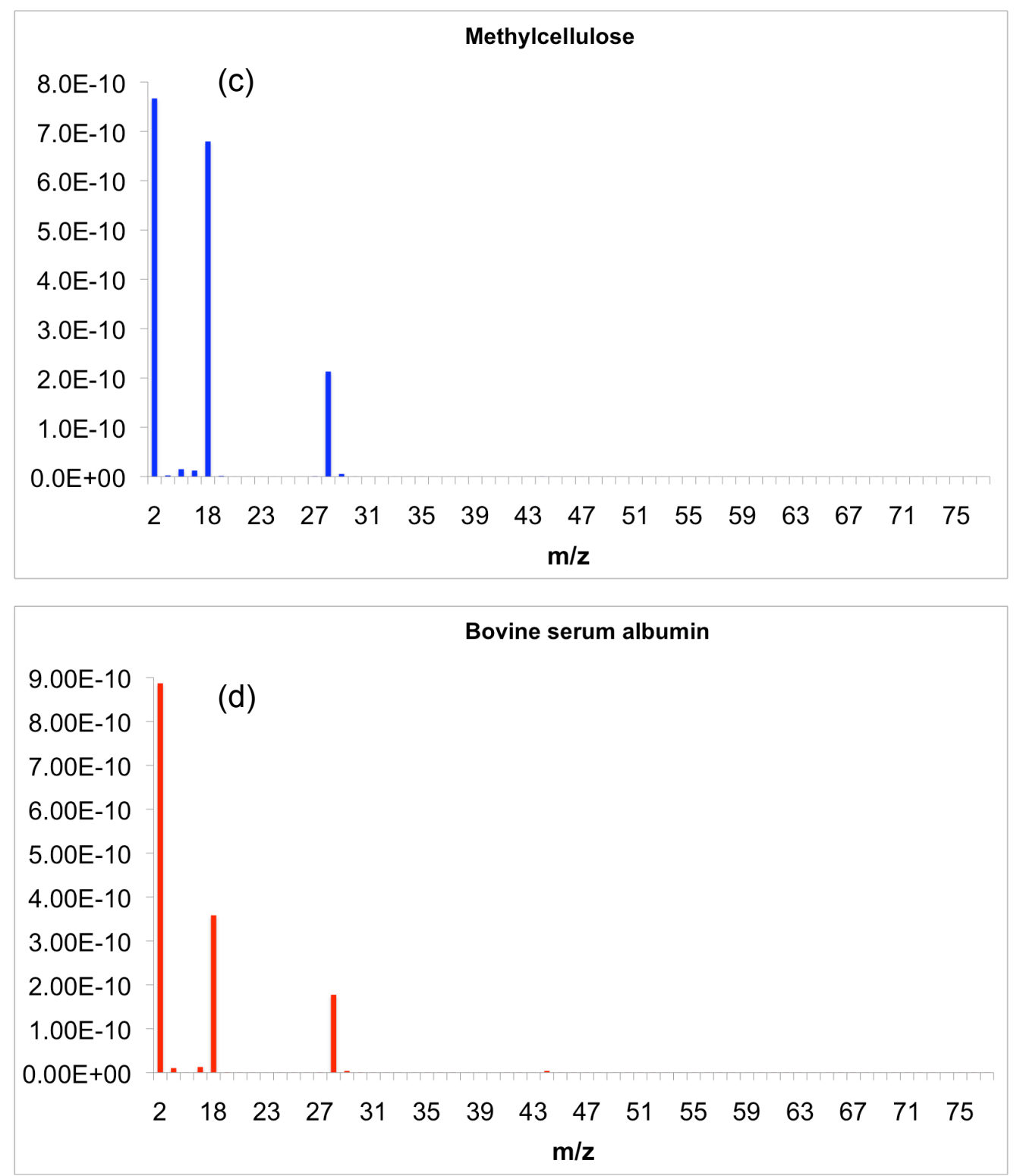
In order to confirm that mass fragment 28 was due to ethene and not nitrogen that may have been adsorbed or trapped inside the hollow SWCNTs, we thermally degraded SWCNTs in He. Theoretically, if the mass fragment 28 was due to internally-sorbed $\mathrm{N}_{2}$, then degradation of the nanotubes would release trapped $\mathrm{N}_{2}$. The amount of trapped $\mathrm{N}_{2}$ should increase with increasing mass of SWCNTs. Degradation of nanotubes in $\mathrm{He}$ should not yield appreciable $\mathrm{C}_{2} \mathrm{H}_{4}$, since there is inadequate $\mathrm{H}_{2}$ to produce $\mathrm{C}_{2} \mathrm{H}_{4}$ from solid C (i.e., SWCNTs). In contrast, degradation of the SWCNTs in $\mathrm{H}_{2}$ should yield $\mathrm{C}_{2} \mathrm{H}_{4}$ from the reaction of solid $\mathrm{C}$ with $\mathrm{H}_{2}$ to form $\mathrm{C}_{2} \mathrm{H}_{4}$.

Since $\mathrm{N}_{2}$ is always present in this system due to air leaks (the TGA-MS is not designed to be airtight), we monitor the $28 / 40$ ion ratio $\left(\mathrm{N}_{2}\right.$ or $\mathrm{C}_{2} \mathrm{H}_{4} / \mathrm{Ar}$ ratio). This ratio is constant when the only $\mathrm{N}_{2}$ present is due to air, while it will change if there is a production of $\mathrm{N}_{2}$ or $\mathrm{C}_{2} \mathrm{H}_{4}$. 
Figure S12. SWCNT degradation in He does not release $\mathrm{N}_{2}$ and SWCNT degradation in $\mathrm{H}_{2}$ does form $\mathrm{C}_{2} \mathrm{H}_{4}$. (a) Differentiated mass loss thermograms of two different masses of SWCNTs in He and one in $\mathrm{H}_{2}$. (b) A mass loss plot is shown to demonstrate the near complete degradation of SWCNTs in He. (c) The ion ratio of mass 28 to mass 40 is shown, and indicates that no $\mathrm{N}_{2}$ was released during SWCNT destruction in He.
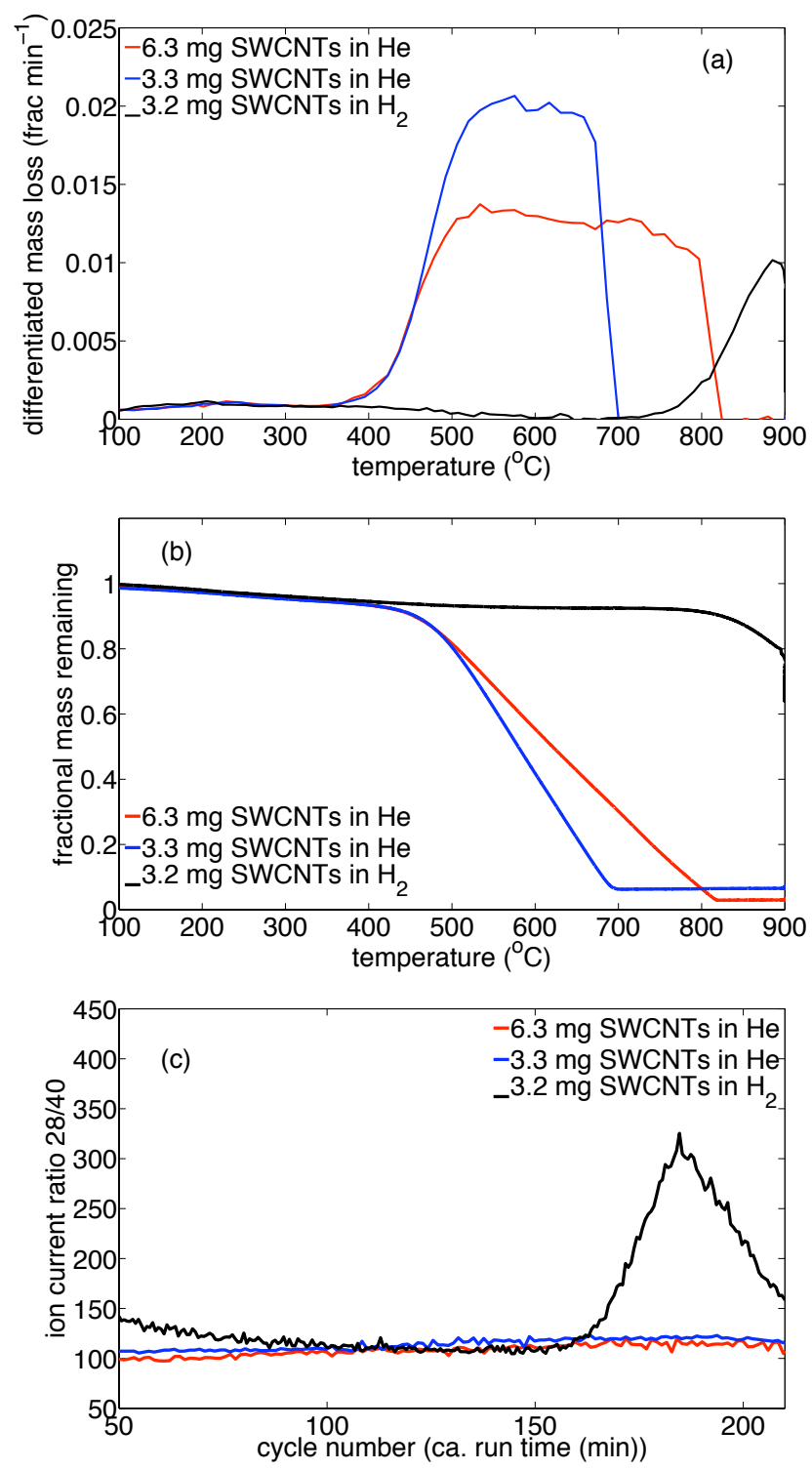
Two different masses of SWCNTs were completely degraded in a He atmosphere (Figure S12). In spite of complete breakdown of the SWCNT, no increase in mass 28, relative to mass 40 , was observed. Thus, significant quantities of $\mathrm{N}_{2}$ were not adsorbed to or trapped in the SWCNTs and could not have contributed to the mass 28 signal observed during HATD of SWCNTs. In contrast, when reacted with $\mathrm{H}_{2}$, SWCNTs did increase the 28/40 ratio, suggesting that $\mathrm{C}_{2} \mathrm{H}_{4}$ was produced from the HATD of SWCNTs. 
Figure S13. Nitrogen leak and ethene calibration during HATD. (a) The mass 28 signal due to $\mathrm{N}_{2}$ decreases throughout the run, with a drop at the start of the temperature program (around cycle \# 60). (b) The response of the mass 28 ion current to increasing ethene concentration. The current when $0 \%(\mathrm{v} / \mathrm{v})$ ethene is introduced drops as the run progresses. All "15 min mean" data points represent the signal averaged over 15 minutes, over which the signal appeared to be stable. (c) The response of the mass 28 ion current to increasing ethene concentration $\left(\mathrm{g} \mathrm{C} \mathrm{mL}^{-1}\right.$ gas). In all of these experiments, $\mathrm{H}_{2}$ was the balance gas.



\section{REFERENCES}

[1] McNichol, A.P.; Osborne, E.A.; Gagnon, A.R.; Fry, B.; Jones, G.A. TIC, TOC, DIC, DOC, PIC, POC- unique aspects in the preparation of oceanographic samples for ${ }^{14} \mathrm{C}$-AMS. Nuclear Instruments and Methods in Physics Res. 1994, B92 (1-4), 162-165. 


\title{
CHAPTER 4: \\ EARLY EVALUATION OF POTENTIAL IMPACTS OF CARBON NANOTUBE SYNTHESIS BY CHEMICAL VAPOR DEPOSITION
}

\author{
By
}

Desirée L. Plata

This work was performed in collaboration with A. John Hart during his post-doctoral appointment at MIT in the Department of Mechanical Engineering and the Department of Aeronautics and Astronautics. The writing and data presented in this chapter are the work of D.L. Plata, principally. 


\begin{abstract}
The carbon nanotube (CNT) industry is rapidly expanding, yet little is known about the potential environmental impacts of CNT manufacture. Historically, delayed evaluation of environmental effects of industrial processes has resulted in costly, and often irreparable, damages to public and ecological health. Here, we evaluate the environmental consequences of a representative multi-walled CNT synthesis by catalytic chemical vapor deposition (CVD). From a heated mixture of ethene and $\mathrm{H}_{2}$, we identified and quantified over 45 side-products formed by thermal treatment of the reactant gases, including methane, volatile organic compounds (VOCs), and polycyclic aromatic hydrocarbons (PAHs). Several environmental concerns resulted, including effects from discharges of the potent greenhouse gas, methane (up to $1.7 \%$ ), and toxins such as benzene and 1,3-butadiene (up to 36,000 ppmv). Extrapolating these data to future CNT production volumes, we estimate that contributions to VOC and PAH emissions may become important on local scales, but will be small compared to national industrial sources. As a first step toward reducing wasteful and unwanted emissions, CNT length was measured in situ, and select thermally generated compounds correlated with CNT growth rate. This suggests that important compounds could be delivered to the catalyst without thermal treatment, thus eliminating the most energetically expensive component of CVD synthesis (heating reactant gases) and minimizing the formation of unintended by-products.
\end{abstract}

\title{
INTRODUCTION
}

Recent reports have highlighted the urgent need for improved understanding of the environmental, health, and safety (EHS) risks associated with nanomaterials and their fabrication $^{1-3}$. Historically, chemical manufacturing procedures have been designed to maximize material performance, minimize production costs, and accelerate the delivery of products to market. However, little attention has been devoted to environmental 
impact(s) mitigation during the design phase of these materials and their production processes. This practice has resulted in severely delayed recognition of malignant environmental and public health effects; and at an advanced stage of industrial development, after substantial investment, it is very difficult to arrest, let alone rectify, the resultant damages. As exemplified by other industrially important materials, retarded identification of undesirable environmental impacts has necessitated costly reparation and remediation efforts (e.g., perfluorooctanoic acid from Teflon ${ }^{\circledR}$ manufacture) and ultimately resulted in product bans and re-development of synthetic technologies ${ }^{4-6}$. Many industrial chemicals follow this pattern of initially rapid production, then recognition of malignant environmental or public health effects, subsequent product bans, and finally, replacement by new technologies on a "few decades" timeframe ${ }^{7}$. Indeed, if history provides a model, then ignoring environmental impacts will all but guarantee the pre-mature departure of promising industries. Aware of this pattern and poised in an aggressively expanding industry, nanoscientists have a unique opportunity to alter the future approach to the development of all materials: by incorporating environmental objectives early in the design phase of each novel chemical process. In doing so, they will not only prevent unnecessary harm to the Earth and human health, but will also preserve the public's confidence ${ }^{8}$ in a field that promises many far-reaching benefits to society.

While the advantages of early efforts to incorporate environmental criteria along with performance and cost metrics in nanomaterial manufacture optimization are quite clear, the approach can be complex. Dahl et al. ${ }^{9}$ suggested an adaptation of the twelve principles of green chemistry ${ }^{10}$ for the design of nanomaterials and their production processes. This includes planning for (1) safer nanomaterials, (2) reduced environmental impact, (3) waste reduction, (4) process safety, (5) materials efficiency, and (6) energy efficiency $^{9}$. Objective 1 relies on structure-activity toxicity information, which will require careful study of a broad spectrum of well-characterized materials in a variety of biological systems ${ }^{1,11}$. Thus, it will likely be several more years before adequate understanding enables the a priori design of safer materials. However, objectives 2 
through 6 can be addressed at the inception of a nanomaterial's fabrication technique. A natural consequence of parallel optimization of environmental and material parameters is the requirement of an interdisciplinary team of researchers (Figure 1). Materials scientists guide the development of the nanomaterial for directed application(s) and offer insight to the materials' intended uses and potential production volume. The latter data are essential to an accurate projection of a material's life cycle impacts. Armed with this information, an environmental chemist can then evaluate the process from chemical mass balance and emission perspectives and offer approaches to limit waste (both in starting materials and effluent streams) and maximize mass conversion and energy efficiency. Then, these data must be fed back to the material engineer to minimize the internal (e.g., manufacture expenses) and external costs (e.g., future remediation expenses) of product production. This iterative, interdisciplinary approach can be used to guide choices for large-scale synthesis, allowing nanomaterial manufacture to develop unlike the majority of industrial chemicals in our history: with "up front" consideration of environmental and public health impacts prior to the observation of a malignant effect.

To provide a model for this co-operative approach, we evaluated a representative synthesis for a promising nanomaterial, vertically aligned multi-walled carbon nanotubes (VA-MWCNTs). While there are several approaches to manufacturing carbon nanotubes (CNTs), including laser-ablation and arc-discharge, the preferred method for largevolume production is catalytic chemical vapor deposition. Typical CVD procedures for CNT growth require heating a carbonaceous gas to high temperature $\left(500-1200^{\circ} \mathrm{C}\right)^{14,15}$ in a reducing or inert atmosphere (e.g., $\mathrm{H}_{2}, \mathrm{He}$, or $\mathrm{Ar}$ ) and subsequent reaction of the heated mixture at a nanoparticle surface (e.g., $\mathrm{Fe}, \mathrm{Co}, \mathrm{Ni}, \mathrm{Co} / \mathrm{Mo})$, which templates $\mathrm{CNT}$ growth. In combustion systems (when oxygen is present), the thermal treatment of carbon-based gases promotes radical formation and recombination reactions that result in the production of other volatile organic compounds (VOCs), polycyclic aromatic hydrocarbons (PAHs), and amorphous carbon or soot ${ }^{16-19}$. Similar mechanisms may occur in oxygen-free systems, but there is limited theoretical and experimental data to support this. Thus, one cannot rely on existing reaction kinetics models (e.g., derived 
from the combustion literature) to predict the evolution of by-products in a CVD reactor for CNT production.

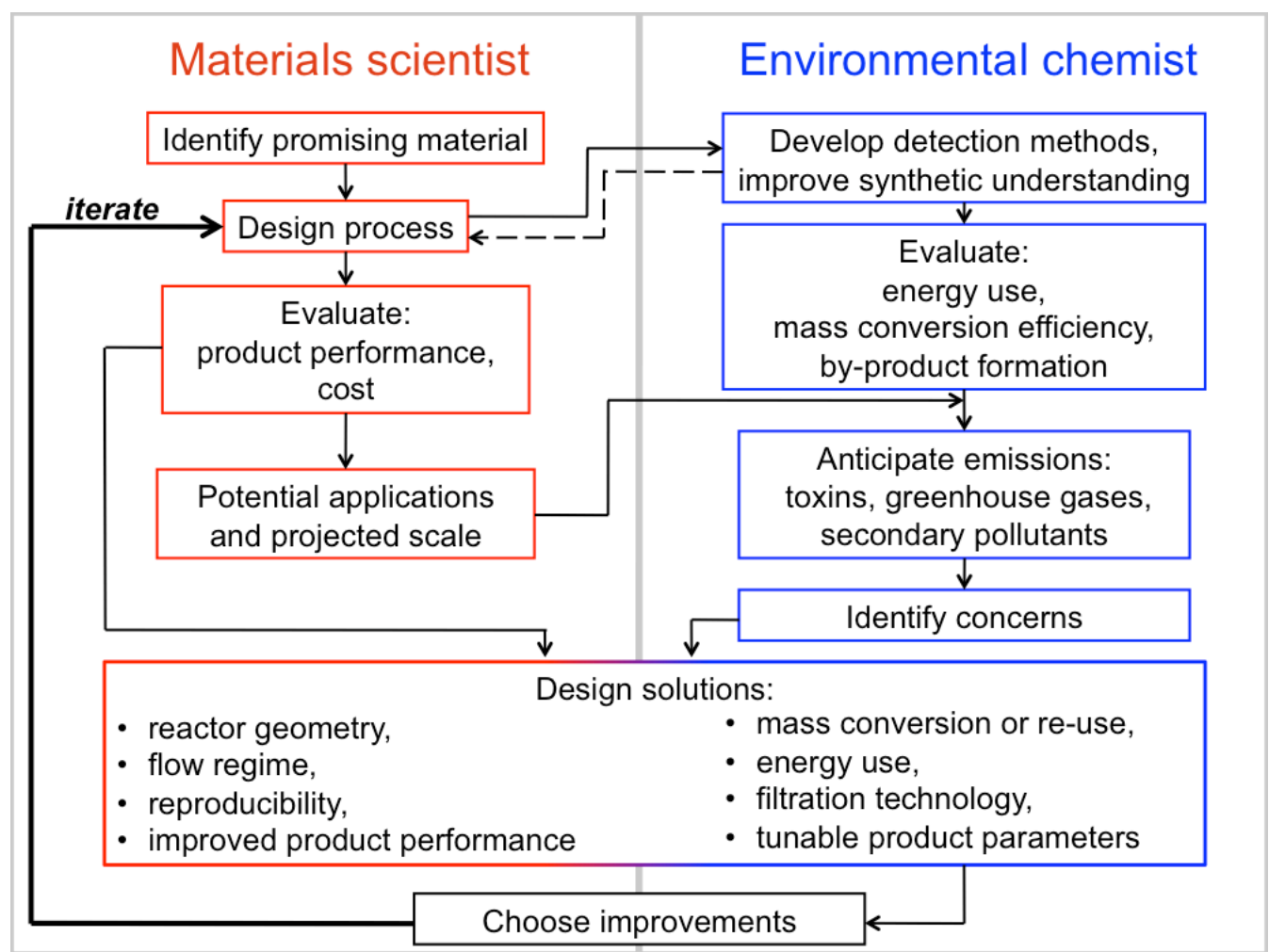

Figure 1. Co-operative approach to material design: incorporating performance, cost, and environmental objectives. Ideally, a collaboration that includes (but is not limited to) materials scientists and environmental chemists begins early in a novel chemical's development on a small-scale, model system. Depending on the fluidity of the synthetic approach, environmental chemists can join the design process even after initial practice has been established (dashed line). However, involvement following substantial and irreversible investments on the part of a manufacturer is non-ideal. Additional team members can include energy use experts ${ }^{12}$, risk analysts ${ }^{13}$, process engineers, toxicologists, and synthetic chemists. 
Nevertheless, some of the compounds formed in reducing or inert gas conditions may present environmental and public health concerns. In particular, photoactive VOCs have long been recognized as contributors to smog and lower atmosphere ozone formation, thereby exacerbating respiratory diseases (e.g., 1976 report from the National Academy of Sciences ${ }^{20}$ ). Furthermore, select PAHs have long been known to be carginogenic, and soot influences public respiratory health ${ }^{2 l}$ as well as the radiative heat balance of the atmosphere. In order to prevent the release of these materials, many CVD effluents pass through "environmental scrubbers" (e.g., paraffin or water bubblers $\left.{ }^{14}\right)$, but short contact times and low partition coefficients may not efficiently capture volatile components. Earlier this year, the Environmental Protection Agency (EPA) strengthened air quality standards ${ }^{22}$ and several industrialized cities (e.g., Houston, TX and Los Angeles, CA $)^{23}$ already exceed these limits. To avoid these potential problems, we must acquire quantitative knowledge of $\mathrm{VOC}, \mathrm{PAH}$, and particulate matter generated during CNT manufacture and adjust synthetic conditions or improve filtration to reduce such pollution.

To date, publicly available effluent analyses have relied on relatively insensitive techniques, such as residual gas analysis ( $c a$. ppm detection limits) ${ }^{24-26}$. For example, online analysis of an ethyne (acetylene)-based CNT production revealed a handful of incompletely identified by-products: cyclohexane, a "cyclopentane fragment," and a "hexane fragment"26. An offline analysis of a different ethyne-based CNT synthesis identified methane and several unsaturated hydrocarbons (specifically, ethene (ethylene), 1-buten-3-yne, pent-3-en-1-yne, two hexadiene-yne isomers, benzene, toluene, and cyclooctatetrene $)^{27}$. However, trace gas components $(<\mathrm{ppm})$ are overlooked by these methods, and these by-products may become important as the industry continues to grow. For example, annual production of CNT powders already exceeds $300 \operatorname{tons}^{28}$ (ton $=10^{6}$ $\mathrm{g}$ ) and is expected to double approximately every two years ${ }^{29}$. If CVD processes have a $3 \%$ atom efficiency ${ }^{30}$, then 10,000 tons of feedstock gas will be used for the present production volume. A sub-ppm contaminant in this effluent (that is not captured before 
release) could be discharged to the atmosphere in large volumes, approximately 3.2 tons, within ten years.

To avoid environmental damages from by-products and improve resource use in VA-MWCNT production, we quantified and identified carbonaceous compounds formed by the thermal treatment of reaction gases $\left(\mathrm{C}_{2} \mathrm{H}_{4} / \mathrm{H}_{2}\right)$. In our CVD reactor (Figure 2), the growth gas mixture was heated and cooled ("pre-heated") before it was impinged on the VA-MWCNT catalyst, which was heated independently on a silicon platform in a closed quartz tube. This physical separation de-convoluted thermal effects on the feedstock gas from those on the metal catalyst and enabled investigation of the two critical processes in isolation $^{31}$. We previously showed that pre-heating is necessary for rapid growth of VAMWCNTs, enhancing the growth rate by over $2000 \%$ compared to when the gas is heated only at the catalyst ${ }^{32}$. VOCs were collected downstream of the pre-heater and upstream of the catalyst, and the reported gas composition represents effects of the thermal treatment only. Simultaneously, we measured VA-MWCNT height in situ using a laser displacement sensor ${ }^{32,33}$. The mixture of thermally generated carbonaceous material was complex and included many VOCs and PAHs, some of which may diminish CNT quality $^{31}$ and present environmental or toxicology concerns. Here, we estimate the potential contribution of two anticipated VA-MWCNT-based products, flat-panel displays and thermal interface materials, to current national and/or local emissions of VOCs and PAHs. With these results, we can begin to co-optimize the synthetic process by (1) minimizing unwanted side-products, (2) minimizing cost by selecting potent reagent gases that require minimal thermal treatment, and (3) maximizing production growth rates.

This is the first step in an iterative process, where we identify opportunities to reduce environmental impact, minimize waste, improve process safety (for the public and occupational population), and maximize materials and energetic efficiency (objectives 26, as described by Dahl et al. ${ }^{9}$ ). Subsequent studies will be required to demonstrate the effectiveness of our suggested measures, but we provide a critical foundation that is 


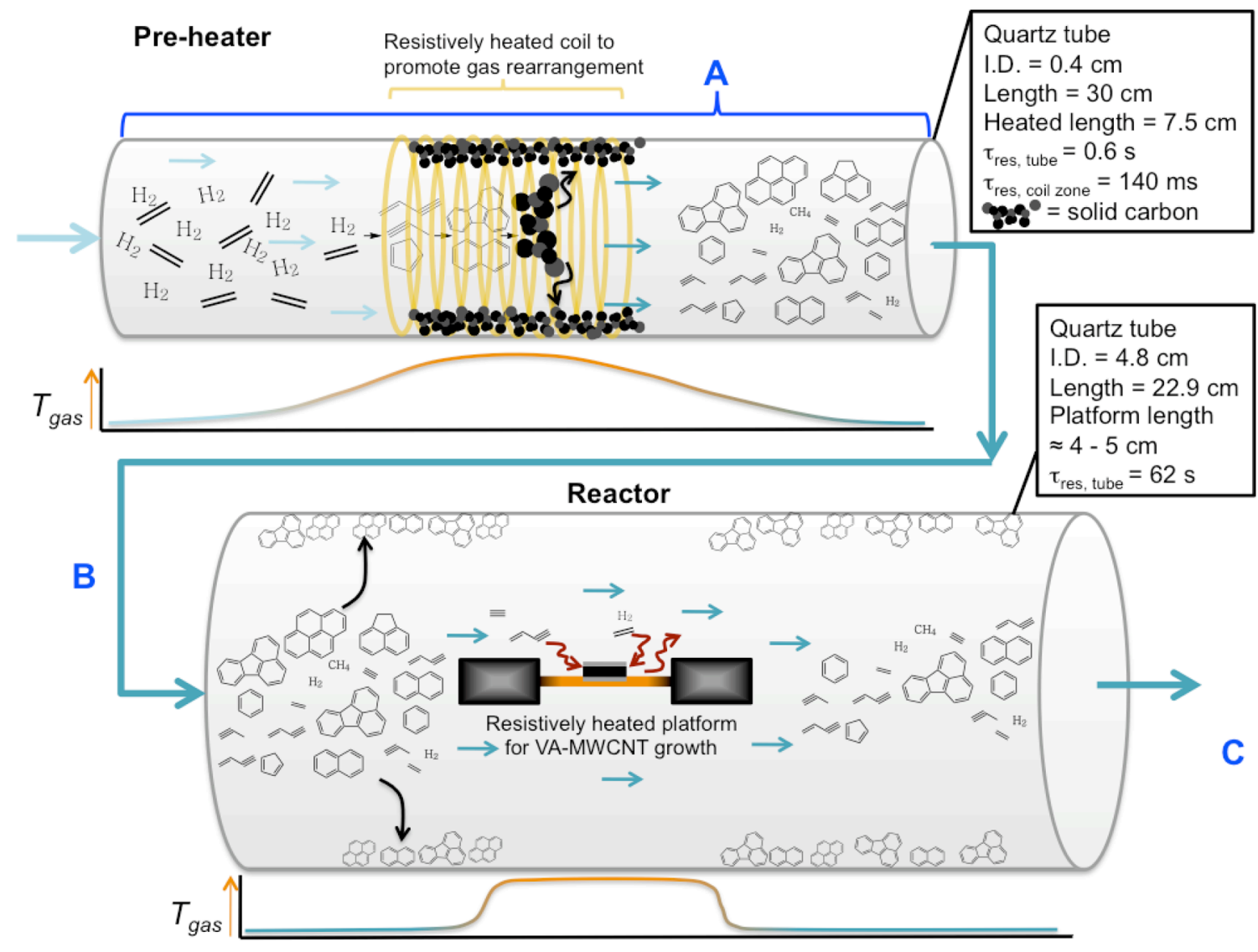

Figure 2. Reactor design and sampling of thermally generated species during transport through a two-stage CVD process, where the gas was first thermally treated in the pre-heater. Chemical structures, particles, and quartz tubes are not drawn to scale. Hydrogen and ethylene were introduced to a narrow-diameter $(4 \mathrm{~mm})$ pre-heater tube that was wrapped with a resistively heated coil and covered with insulating material (insulation not shown). At high temperatures (e.g., $690-1200^{\circ} \mathrm{C}$ ), ethylene and hydrogen rearrange and form other molecules (e.g., methane, 1,3-butadiene, benzene, and fluoranthene) and soot-like particles. Solid carbon deposits formed in the heated zone of the pre-heater tube, but were not deposited significantly outside of the heated zone. (Sampling zone A was sectioned along the flow axis prior to quantification of solid carbon deposits). VOCs and PAHs survived transit through the pre-heater tube and were delivered to a room-temperature reactor. Here, PAHs were lost from the reactant stream (see Supporting Information) due to sorption to the cool quartz walls of the reactor. Some PAHs were transported out of the reactor as effluent. VOCs were not retained on the reactor walls, and comparisons at sampling points $\mathrm{B}$ and $\mathrm{C}$ did not show distinct differences. VOC and PAH abundances presented in subsequent figures of the text represent samples collected at sampling points B and C, respectively. Both PAHs and VOCs were available for carbon nanotube growth on the resistively heated platform, which supported a VA-MWCNT catalyst substrate. 
needed to advance CNT production and prevent unnecessary damage to the environment, public health, and the public's opinion of nanotechnology as a whole.

\section{RESULTS AND DISCUSSION}

Improving CNT synthetic efficiency from an industrial perspective and minimizing environmentally undesirable side products requires a deeper understanding of the chemical reactions occurring in the gas phase of CVD reactors. Here, we sought to identify and quantify the volatile, semi-volatile, and solid products formed as a function of pre-heater temperature and reaction gas composition (e.g., $\mathrm{H}_{2} / \mathrm{C}_{2} \mathrm{H}_{4}$ ratio). Simultaneously, we aimed to optimize the synthetic performance by monitoring in situ CNT growth kinetics.

VOCs formed during VA-MWCNT synthesis: Effects of pre-heater temperature. The thermal pretreatment of VA-MWCNT reactant gases $\left(\mathrm{C}_{2} \mathrm{H}_{4} / \mathrm{H}_{2}=70 / 330\right.$ standard cubic centimeters per minute $(\mathrm{sccm})$ ) resulted in the formation of many VOCs (Figure 3 ). None of these VOCs have been reported previously in an ethene-based CVD synthesis of CNTs, but methane, ethane, benzene, and 1-buten-3-yne (vinyl acetylene) have been detected in the effluents of an ethyne-fed CVD reactor ${ }^{27}$. Relatively small increases in the pre-heater current lead to order-of-magnitude increases in the abundances of methane, benzene, propyne, and pentane; whereas ethane, 1,3-butadiene, and 1,2-butadiene were less sensitive to thermal treatment. VOC concentrations ranged from $4.7 \pm 0.3 \mathrm{ppmv}$ (1,3-cyclopentadiene) to $8,700 \pm 500 \mathrm{ppmv}$ (ethane) at the lowest pre-heater current $(1.5$ $\left.\mathrm{A}, 690^{\circ} \mathrm{C}\right)$ and from $17.6 \pm 0.1 \mathrm{ppmv}(1,3$-cyclopentadiene) to $17,000 \pm 700 \mathrm{ppmv}$ (methane) at the highest pre-heater current $\left(2.0 \mathrm{~A}, 1040^{\circ} \mathrm{C}\right)$. Increasing pre-heater temperature clearly accelerated VA-MWCNT growth (Figure 4), and this suggests some of the VOCs may accelerate VA-MWCNT growth. 


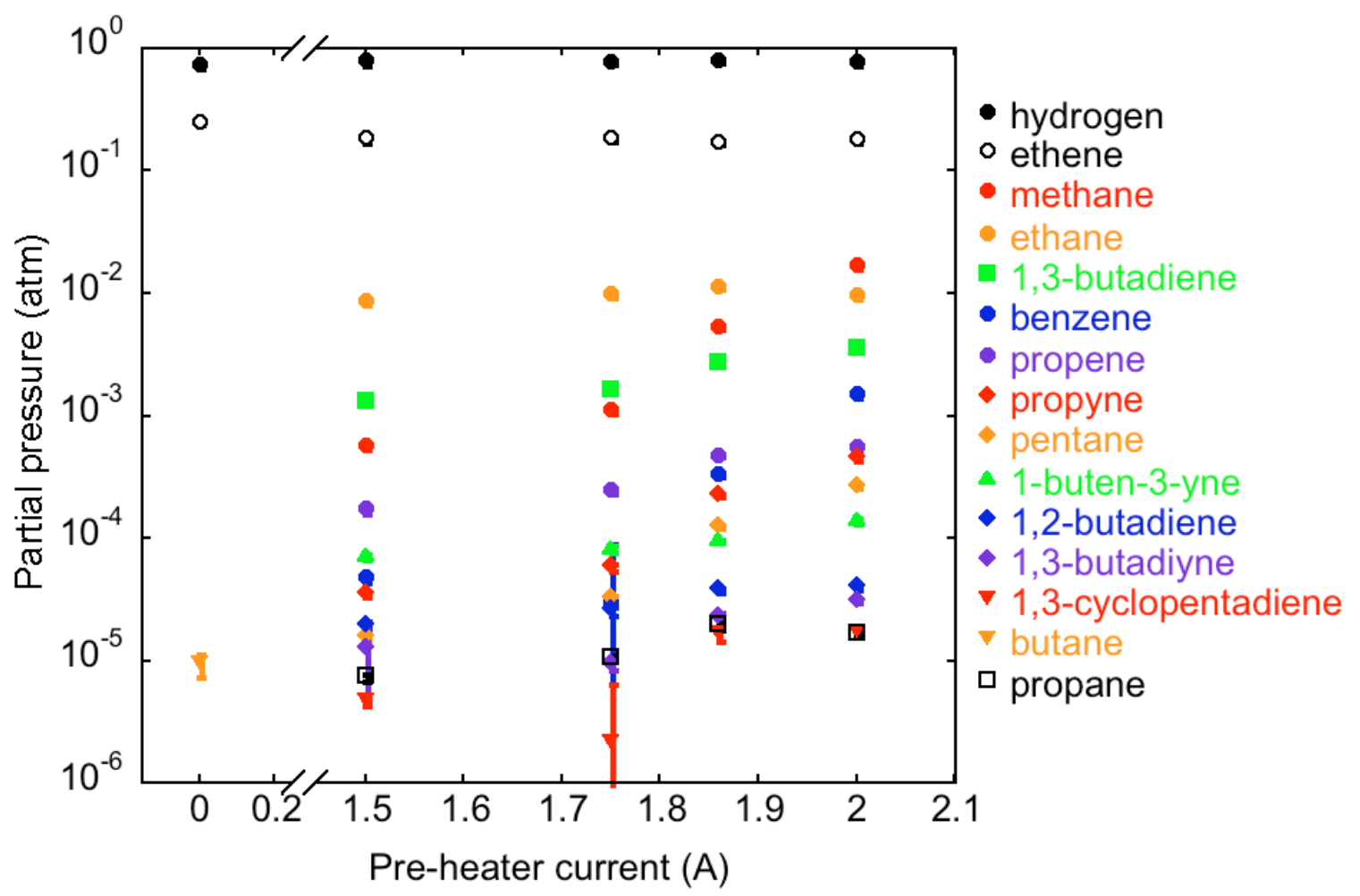

Figure 3. VOC abundance as related to pre-heater current, a surrogate metric for preheater temperature. Pre-heater current was related to temperature approximately as follows: $1.5,1.75,1.86,2.0 \mathrm{~A}=690,860,920,1040^{\circ} \mathrm{C}$, respectively. Error bars represent one standard deviation on triplicate measurements of the same samples, and non-visible error bars are smaller than the data marker. 

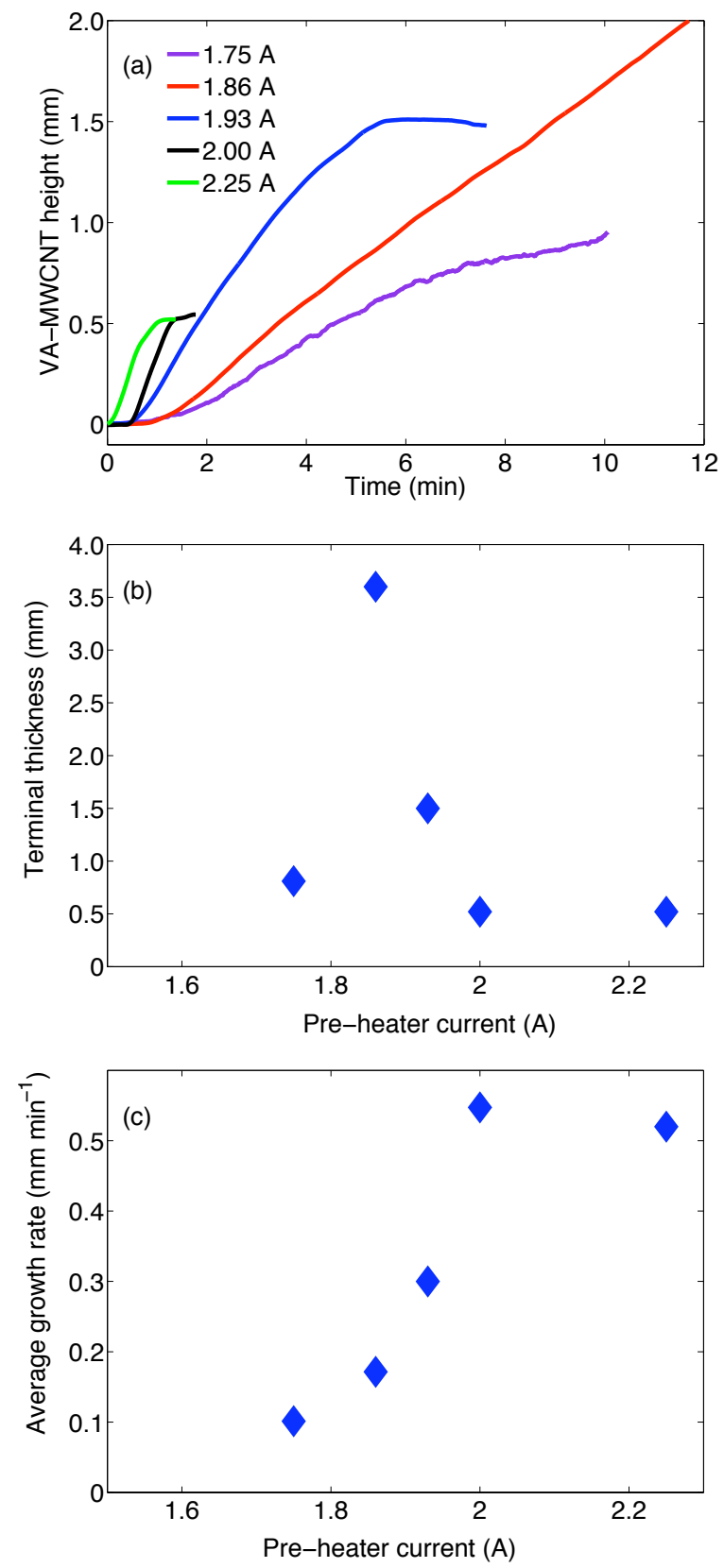

Figure 4. Thermal pre-treatment of reactant gases $\left(\mathrm{H}_{2} / \mathrm{C}_{2} \mathrm{H}_{4}\right)$ affected VA-MWCNT growth rate and terminal height. (a) Tube growth as a function of pre-heater current. (b) The terminal height of the VA-MWCNT forest exhibited a maximum at $920^{\circ} \mathrm{C}$, while (c) the growth rate reached a plateau with increasing temperature. Pre-heater current was related to temperature as follows: $1.75,1.86,2.0,2.25 \mathrm{~A}=860,920,1040,1200^{\circ} \mathrm{C}$, respectively. 
Although the effluent mixture was complex, some thermally generated compounds were correlated to the increased growth rate observed at higher pre-heating temperatures. There were strong correlations between the growth rate and the partial pressures of methane, benzene, and 1-buten-3-yne (each with correlation coefficients of $0.99 ; n=3$ ). In our system, only the abundances of methane and ethane were sufficient to account for the mass of VA-MWCNTs formed. Although less abundant, the unsaturated compounds could accelerate VA-MWCNT growth by co-polymerizing with ethene at the metal catalyst, an effect that has been observed in polyethylene synthesis $^{34-36}$.

Interestingly, the abundances of the same VOCs did not correlate with the absolute CNT height, which maximized at the 1.86 A pre-heater current and decreased sharply at $2.00 \mathrm{~A}\left(3.6\right.$ and $0.52 \mathrm{~mm}, \mathrm{~T}=920$ and $1040^{\circ} \mathrm{C}$, respectively). Instead, the terminal height was directly correlated to ethane abundance (correlation coefficient of $0.97 ; n=3$ ), suggesting that reducing conditions that lead to ethane formation also play a role in sustaining catalyst activity.

Since production of such VOCs could be environmentally problematic (see below) and wasteful, these results suggest it may be possible to selectively deliver only the appropriate reactants to the catalyst, rather than relying on thermal techniques to generate a suite of diverse reactants. Consequently, we would avoid the thermal pretreatment step, and thus prevent the formation of potentially interfering products (e.g., soot), minimize unnecessary toxin formation, and eliminate the most expensive component of VA-MWCNT synthesis (heating the reactant gases ${ }^{37}$ ). Furthermore, this technique could dramatically improve the carbon-to-VA-MWCNT mass conversion efficiency.

\section{VOCs formed during VA-MWCNT synthesis: Effects of hydrogen gas content.} Several reports suggest that the partial pressure of hydrogen $\left(\mathrm{pH}_{2}\right)$ in the CNT feedstock gas can influence the diameter ${ }^{38}$ and purity of $\mathrm{CNTs}^{39}$. Hydrogen species $\left(\mathrm{H} \bullet, \mathrm{H}_{2}\right)$ play critical roles in gas-phase radical reactions ${ }^{16,17,19,40}$, so it is reasonable to expect changes in $\mathrm{pH}_{2}$ to alter $\mathrm{VOC}$ formation. At $0 \mathrm{pH}_{2}$, the $\mathrm{VOC}$ abundances were relatively low 
(normalized to total carbon abundance; Figure 5). As $\mathrm{pH}_{2}$ increased, ethene, of course, decreased chiefly by dilution. Ethane and methane's abundances relative to ethene increased with $\mathrm{pH}_{2}$, while all the other VOCs showed a maximum presence at mid $\mathrm{pH}_{2}$. As observed in earlier tests of the effects of temperature, ethane abundance was correlated to VA-MWCNT terminal height (230, 1300, 1950 um for $0,0.5$, and $0.8 \mathrm{~atm}$ $\mathrm{H}_{2}$, respectively; correlation coefficient of $0.98, n=3$ ). Thus, reducing conditions substantially influenced the VOCs formed, as well as the terminal height of the product.
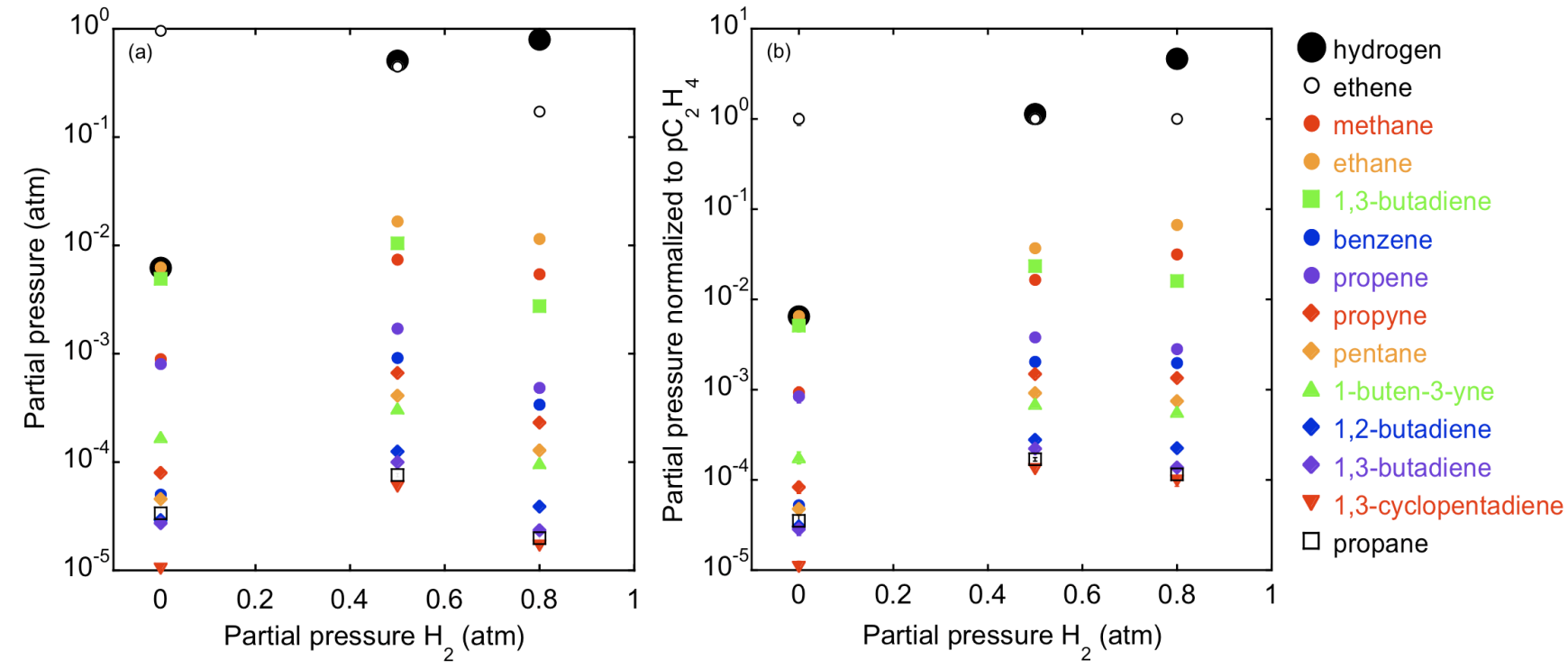

Figure 5. The abundance of VOCs varies with the partial pressure of hydrogen. Individual VOCs concentrations are shown (a) in absolute partial pressure or (b) normalized to the partial pressure of ethene. Note that some $\mathrm{H}_{2}$ was present in the postpre-heater gas mixure of the $0 \mathrm{pH}_{2}$ case, as some $\mathrm{H}_{2}$ is generated from the thermal decomposition of ethene ${ }^{16,17}$. Error bars show standard deviation of triplicate analyses of the same sample. All experiments were conducted with a pre-heater current of $1.86 \mathrm{~A}$ $\left(920{ }^{\circ} \mathrm{C}\right)$. 


\section{Safety concerns and environmental implications of extrapolating VOC}

emissions to industrial scale. While pre-heating and varying the composition of the $\mathrm{C}_{2} \mathrm{H}_{4} / \mathrm{H}_{2}$ feedstock can enable tuning of VA-MWCNTs growth rate and height, several of the thermally generated VOCs can also have undesirable environmental and public health effects. Of particular concern are: (1) methane, a potent greenhouse gas, (2) photoactive VOCs, which contribute to smog formation, and (3) benzene and 1,3-butadiene, which are regulated as hazardous air pollutants and occupational chemical hazards (by the EPA and the Occupational Safety and Health Administration (OSHA), respectively). At all of the tested pre-heater currents, benzene concentrations were over 40 times higher than time-weighted average permissible exposure limits (TWA PEL; 1 ppmv). Similarly, 1,3butadiene concentrations exceeded the TWA PEL (1 ppmv) by over a factor of 1000, increasing from $1,300 \pm 100 \mathrm{ppmv}$ to $3,600 \pm 200 \mathrm{ppmv}$ over the tested pre-heater range. At the highest current, the 1,3-butadiene and benzene contents of the effluent stream are considered immediately dangerous to life and health (IDLH; limits of 2000 and 500 ppmv, respectively ${ }^{41}$ ). Typically, great care is taken to protect employee health, and effluents are vented through fume hoods. However, if the reactant mixture is discharged to the atmosphere without sufficient treatment, industrial-scale CNT synthesis may result in unacceptable environmental and public health consequences, and thus threaten the ultimate success of the CNT market ${ }^{2}$.

As the commercial market for CNT-based products is relatively nascent, there is a great deal of uncertainty surrounding the magnitude of production and the details of industrial-scale synthesis. (For example, CVD is favored for large-scale CNT production, but ethene may not be a preferred carbon feedstock). Acknowledging the inherent uncertainty in the future magnitude of the industry, we estimated the prospective contributions of CNT manufacture to the anthropogenic VOC flux, focusing on two potential large-scale applications of VA-MWCNTs: flat panel displays (FPDs) and 
thermal interface materials (TIMs). In our reactor ${ }^{\mathrm{a}}, 0.5 \mathrm{~cm}^{2}$ VA-MWCNTs grew to a height of $2 \mathrm{~mm}$ and released $0.70 \pm 0.04 \mathrm{~g}$ VOCs (at the 1.86 A pre-heater current, $\mathrm{T}=$ $\left.920^{\circ} \mathrm{C} ; \mathrm{pH}_{2}=0.8 \mathrm{~atm}\right)$. We scale this effluent mass to account for the shorter nanotubes and larger areas required by each respective VA-MWCNT application (see Supporting Information). For the sake of argument, we assume that VA-MWCNT use will reach the same magnitude in FPDs as liquid crystal displays (LCDs) and that VA-MWCNTs will be used as TIMs in home computers ${ }^{\mathrm{b}}$. If we scale VA-MWCNT production to account for the annual LCD and home computer CPU (central processing unit) sales (in total surface area, see Supporting Information) $)^{43,44}$, then roughly $50 \pm 3 \mathrm{Gg} \mathrm{VOCs} \mathrm{yr}^{-1}$ and $0.40 \pm 0.02 \mathrm{Gg}$ VOCs $\mathrm{yr}^{-1}$ will be generated by FPD and TIM technologies, respectively (Table 1 , note $1 \mathrm{Gg}=10^{9} \mathrm{~g}$ ). These emissions would represent between 0.34 and $0.002 \%$ of all the anthropogenic VOC emissions from all other sources in the United States (16 ${\operatorname{Tg~} \mathrm{r}^{-1}}^{45}$, including industrial, residential, and automotive sources. The dramatic difference between the FPD and TIM estimates results from the distinct surface areas required for each, the former requiring a much larger area of VA-MWCNTs than the latter $\left(3.8 \times 10^{11}\right.$ and $2.7 \times 10^{9} \mathrm{~cm}^{2}$, respectively). While the total VOC output influences tropospheric ozone formation, which is regulated by the EPA, each VOC has unique photoreactivity, toxicity, and greenhouse gas potential. Extending these estimates to individual compounds, we predict $1.00 \pm 0.03 \mathrm{Gg} \mathrm{yr}^{-1}$ methane, $2.0 \pm 0.1 \mathrm{Gg} \mathrm{yr}^{-1} 1,3-$ butadiene, and $0.30 \pm 0.01 \mathrm{Gg} \mathrm{yr}^{-1}$ benzene would be generated from VA-MWCNT synthesis for FPDs. VA-MWCNT manufacture for TIMs would generate lesser emissions; $7.0 \pm 0.2 \mathrm{Mg} \mathrm{yr}^{-1}$ methane, $10 \pm 1 \mathrm{Mg} \mathrm{yr}^{-1}$ 1,3-butadiene, and 2.0 $\pm 0.1 \mathrm{Mg} \mathrm{yr}^{-}$ ${ }^{1}$ benzene (note: $1 \mathrm{Mg}=10^{6} \mathrm{~g}$ ). On a national scale, these contributions are small

\footnotetext{
${ }^{a}$ This reactor is considered efficient (high mass conversion of feedstock to CNT; here 0.2 $\%$ ) compared to other research-scale reactors, but is likely less efficient than would be expected for an industrial-scale reactor ( $3 \%$ reported for single walled CNT powders). ${ }^{\mathrm{b}}$ Note that LCDs are very competitive in terms of cost and performance, and substantial advances in CNT technology are required before VA-MWCNT FPDs become a commercial reality ${ }^{42}$. VA-MWCNT TIMs are in the early stages of development, but they are very promising. If ultimately successful, their market applications will extend to all electronics, not just home computers.
} 
compared to total annual emissions of methane $\left(25,000 \mathrm{Gg} \mathrm{yr}^{-1}\right)^{46}$ and benzene $((2.4 \mathrm{Gg}$ $\left.\mathrm{yr}^{-1}\right)^{47}$, but can exceed emissions of 1,3-butadiene $\left(0.7 \mathrm{Gg} \mathrm{yr}^{-1}\right)^{47}$ from all other industrial sources. On a local scale, benzene and 1,3-butadiene releases due to VA-MWCNT manufacture for FPDs could surpass or approach the total emissions from residential and industrial releases combined. For example, in Houston, TX (one of the United States' most industrialized cities), total 1,3-butadiene emissions are $0.68 \mathrm{Gg} \mathrm{yr}^{-1}$ and total benzene emissions are $1.7 \mathrm{Gg} \mathrm{yr}^{-148}$. Thus, if $20 \%$ of the VA-MWCNT-based FPDs were manufactured in Houston, 1,3-butadiene outputs would increase by almost $50 \%$ and benzene emissions would increase by over $3 \%$. In contrast, contributions from VAMWCNT-based TIMs would be marginal. Clearly, the relative impact of a CNT-based material is very sensitive to both the size of the market and the specific application requirements (e.g., large mass or surface area), and such estimates should be considered for each novel technology.

Table 1. Projected emissions due to CVD synthesis of VA-MWCNTs for potential applications compared to national and local emissions.

\begin{tabular}{ccccc}
\hline \multirow{2}{*}{ Compound(s) } & \multicolumn{4}{c}{ Annual emissions $\left(\mathrm{Gg} \mathrm{yr}^{-1} ; \mathrm{Gg}=10^{9} \mathrm{~g}\right)$} \\
\cline { 2 - 5 } & FPDs $^{a}$ & TIMs $^{a}$ & Houston, TX & United States \\
\hline$\Sigma$ VOCs & $50 \pm 3$ & $0.40 \pm 0.02$ & $---{ }^{b}$ & $16,000^{c}$ \\
methane $^{d}$ & $1.00 \pm 0.03$ & $0.0070 \pm 0.0002$ & $---{ }^{b}$ & $25,000^{e}$ \\
1,3 -butadiene & $2.0 \pm 0.1$ & $0.010 \pm 0.001$ & $0.68^{f}$ & $0.7^{g}$ \\
benzene & $0.30 \pm 0.01$ & $0.0020 \pm 0.0001$ & $1.7^{f}$ & $2.4^{g}$ \\
\hline
\end{tabular}

${ }^{a}$ Methods used to estimate emissions from VA-MWCNT synthesis for FPDs (flat-panel displays) and TIMs (thermal interface materials) are presented in the Supplemental Information.

${ }^{b}$ Data not available.

${ }^{c}$ Environmental Protection Agency ${ }^{45}$

${ }^{d}$ Methane is not classified as a VOC by the EPA, as it is not photoactive. ${ }^{e}$ Environmental Protection Agency ${ }^{46}$

${ }^{f}$ All emissions sources, Evironmental Protection Agency ${ }^{48}$

${ }^{g}$ All industrial emissions sources, Environmental Protection Agency ${ }^{47}$ 


\section{PAH formation during VA-MWCNT synthesis: Effects of temperature and}

hydrogen gas content. Several PAHs, formed during VA-MWCNT synthesis, survived transit through the quartz reactor (Figure 6, samples collected at point $\mathrm{C}$ in Figure 2).

Our analytical method concentrates hydrophobic PAHs over the course of the reaction, and so PAH concentrations reported here are given relative to the total amount of carbon delivered during the VA-MWCNT growth. Naphthalene was the most abundant PAH at

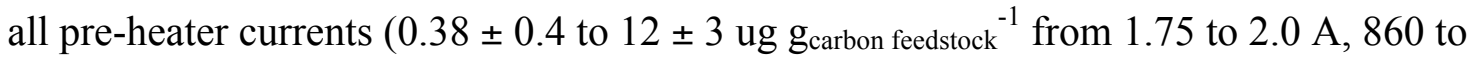
$1040^{\circ} \mathrm{C}$, respectively), and anthracene was the least abundant $(14.2 \pm 0.2$ to $95 \pm 20 \mathrm{ng}$ $\left.\mathrm{g}_{\text {carbon feedstock }}{ }^{-1}\right)$.

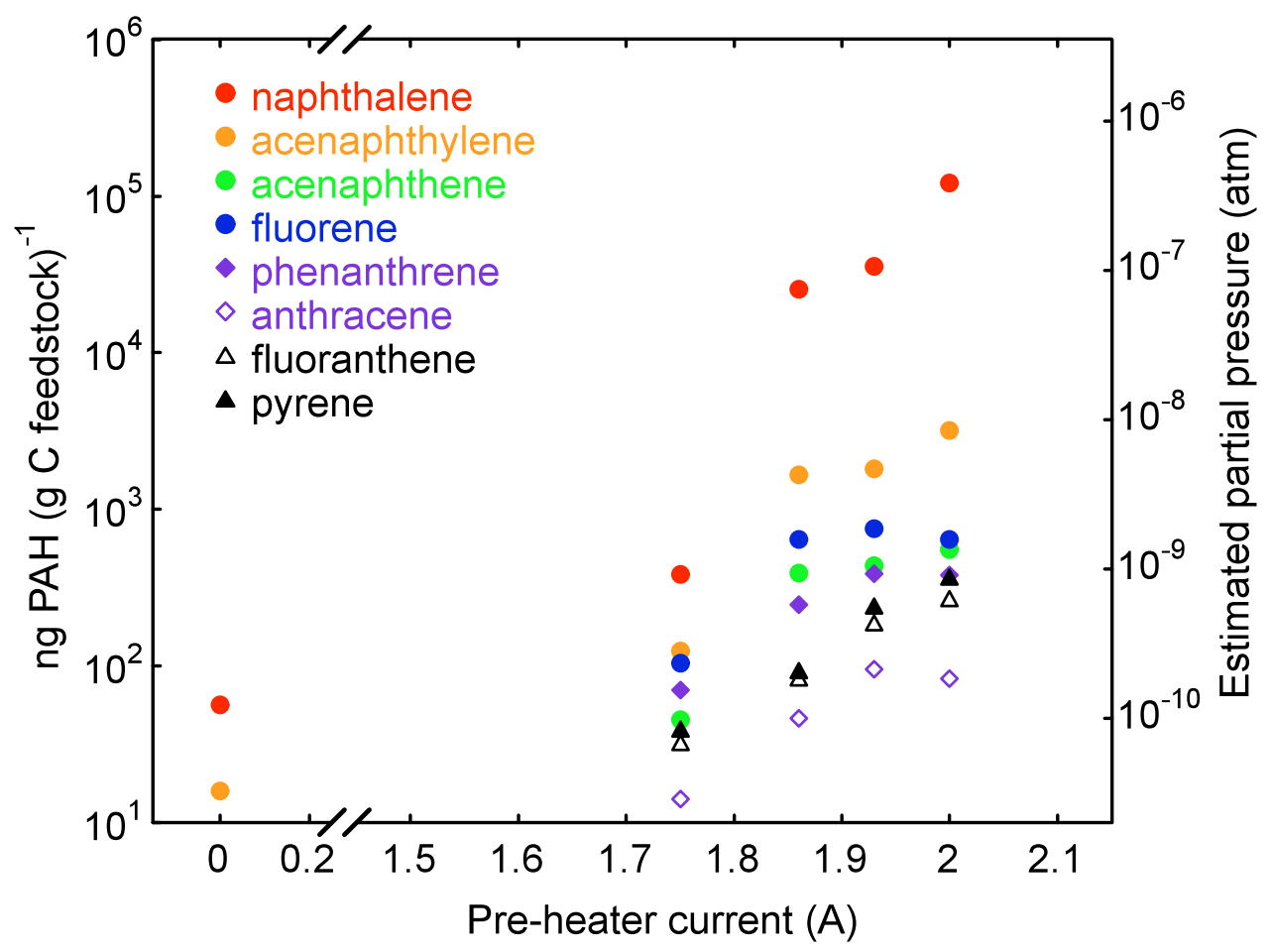

Figure 6. PAH content as related to pre-heater current. The sampling method concentrated PAHs over the course of the reaction, and PAH content is reported relative to the total amount of carbon delivered to the reactor (left-hand axis). Assuming uniform PAH production during the synthesis, we can calculate an average concentration of PAH in the effluent gas (right-hand axis), which enables comparison with NIOSH and EPA regulations. Pre-heater current was related to temperature approximately as follows: 1.75 , $1.86,1.93,2.0 \mathrm{~A}=860,920,970,1040^{\circ} \mathrm{C}$, respectively. 
Individual PAHs increased by orders of magnitude with increasing pre-heater current (Figure 6). Changes in $\mathrm{pH}_{2}$ also influenced the $\mathrm{PAH}$ content of the effluent, where higher $\mathrm{PAH}$ abundances were observed at higher $\mathrm{pH}_{2}$ (Figure $7 \mathrm{~b}$ ). Naphthalene, fluoranthene, and pyrene production were correlated to the increasing VA-MWCNT growth rate (in temperature experiments, correlation coefficients of 0.99, 0.99, 0.99, respectively, $\mathrm{n}=3$ ). Select PAHs are thought to be important for CNT nucleation (e.g., fluoranthene ${ }^{49}$ ), but the role of PAHs in CNT growth (and/or inhibition) has yet to be explored experimentally. To date, on-line analysis of CVD reactants have had limited sensitivity (detection limits in the ppmv range), precluding the identification of trace components. The pre-concentration methods used in this study offer detection limits in the sub parts per trillion $\left(10^{-12}\right)$ range. Without this sensitivity, none of the PAHs formed during the tested syntheses would have been observed. Similarly, if private manufacturers use on-line techniques to monitor toxin formation in their reactors, they may unknowingly release PAHs to the atmosphere.
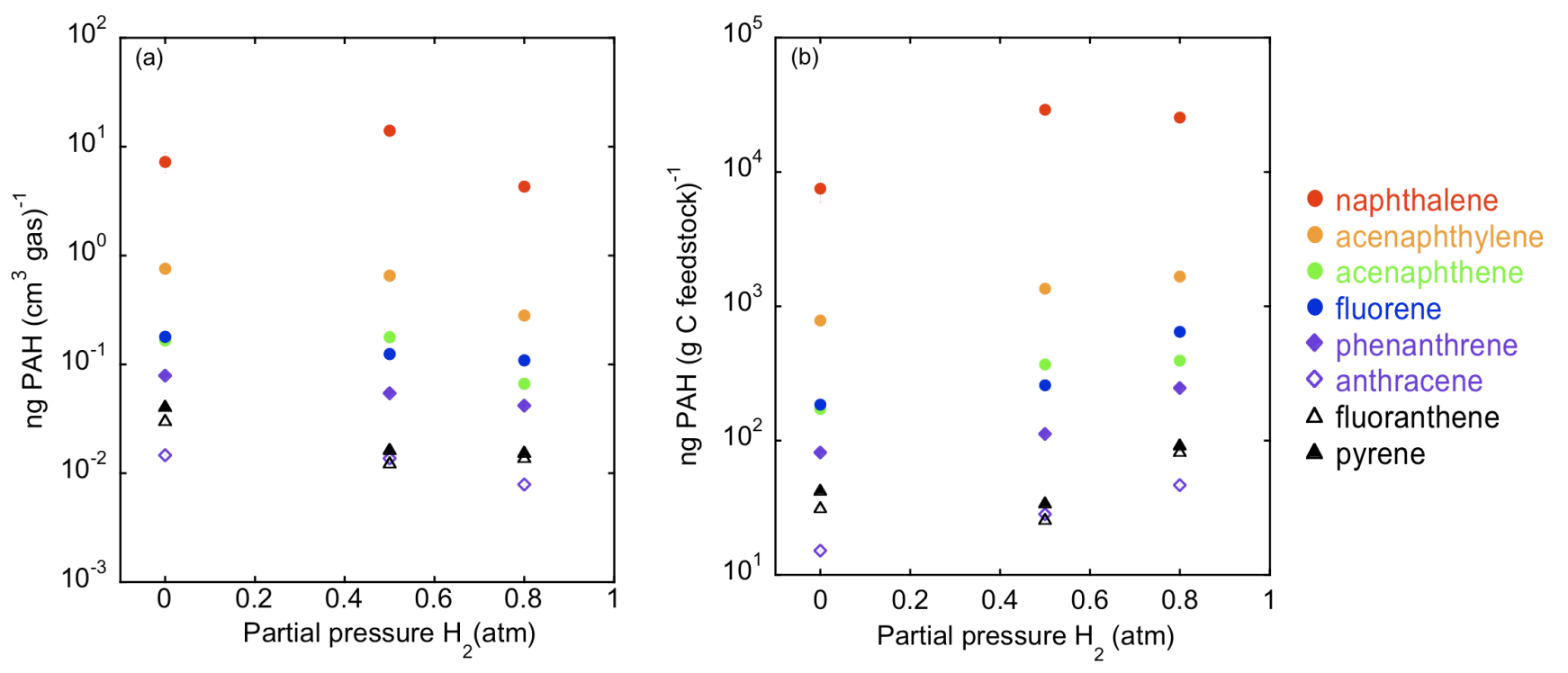

Figure 7. PAH abundances as influenced by the partial pressure of hydrogen. PAH concentration is reported relative to (a) to the total volume of gas filtered by the polyurethane foam and (b) the total amount of carbon delivered to the reactor. Error bars represent one standard deviation on triplicate analysis of the same sample. Invisible error bars are smaller than the symbol. 


\section{PAH safety concerns and environmental implications of extrapolating to}

industrial scale. Many PAHs are toxic and subject to regulation by the EPA and OSHA. To determine if the PAHs formed in the reactor may present an occupational risk, we calculated the average concentration of the PAH in the effluent stream by assuming constant PAH formation over the course of the reaction (Figure 7a). At the highest preheater current, the runtime-average naphthalene content was $0.33 \pm 0.01 \mathrm{ppm}(1.7 \pm 0.1$ $\left.\mathrm{mg} \mathrm{m}^{-3}\right)$. This does not exceed the TWA PEL $(10 \mathrm{ppm})^{40}$, but the total PAH content of the effluent stream $\left(\Sigma \mathrm{PAH} \mathrm{EPApriority}_{\mathrm{N}} 1.8 \pm 0.1 \mathrm{mg} \mathrm{m}^{-3}\right)$ was concentrated relative to engine exhaust $\left(\Sigma \mathrm{PAH} \mathrm{EPApriority}_{\text {0 }} 0.1-0.8 \mathrm{mg} \mathrm{m}^{-3}\right)^{50,51}$. Extrapolating these results (as done before for VOCs), we estimate that future possible PAH emissions from VA-MWCNT synthesis for FPDs and TIMs will be small (15 \pm 0.1 and $0.11 \pm 0.01 \mathrm{Mg} \mathrm{yr}^{-1}$, respectively) compared to national emissions $\left(\geq 16 \mathrm{Gg} \mathrm{yr}^{-1}\right)^{52}$, but may be important on a local scale. If so, it will be necessary to find ways to either limit PAH release to the atmosphere (e.g., by filtration) or minimize PAH formation. Indeed, minimizing PAH formation may prove to be beneficial, not only for the environment, but also for the quality of the CNT product $^{31}$.

There are several sources of PAHs to the environment, and diagnostic PAH ratios have been used to indicate the origin and formation temperature of $\mathrm{PAHs}^{53,54}$. Fluoranthene/pyrene ratios from VA-MWCNT synthesis were between 0.7 and 0.8 , very similar to those found in urban aerosols (0.6 to 0.8), and phenanthrene/anthracene ratios were between 4 and 5, consistent with pyrogenic PAH formation (phenanthene/anthracene $<10)^{55}$. High-temperature processes (i.e., $>$ approximately $800^{\circ} \mathrm{C}$ ) often favor non-alkylated PAH formation, and methylated PAH-to-parent PAH ratios from VA-MWCNT synthesis are low, as expected, decreasing from 0.38 to 0.06 with increasing pre-heater current. While VA-MWCNT-derived PAH ratios are not distinct from other high-temperature processes, there are some unique compounds in the CVD effluent stream that may be useful for tracking emissions. Acephenanthrylene, an isomer of fluoranthene and pyrene, is rarely observed in the natural world ${ }^{56}$. The presence of this unique isomer (see Supporting Information) in VA-MWCNT effluent 
may be useful for tracing CVD reactant streams near the point-of-release. However, the atmospheric lifetime of these chemicals must be explored before they can offer conclusive information about PAH sources in aged environmental samples.

Solid carbon formation during VA-MWCNT synthesis: Effects of temperature and hydrogen gas content. Large PAHs are also pre-cursors to soot ${ }^{57}$, and controlling PAH formation during VA-MWCNT synthesis may allow one to limit the co-production of sooty or amorphous carbon phases, which are interfering contaminants in many CNTbased applications. In this system, carbonaceous solids were deposited on the inner walls of the pre-heater tube, but were not visible in the reactor tube (where VA-MWCNT growth occurred). Solid C deposition increased from 0.2 to $4.0 \mathrm{mg} \mathrm{g}_{\text {carbon feedstock }}{ }^{-1}$ as the pre-heater current increased from 1.75 to $1.93 \mathrm{~A}$ ( 860 to $970{ }^{\circ} \mathrm{C}$, Table 2$)$, but then decreased at the highest pre-heater current $\left(2.6 \mathrm{mg} \mathrm{g}_{\text {carbon feedstock }}{ }^{-1}\right.$ at $\left.2.00 \mathrm{~A}, 1040^{\circ} \mathrm{C}\right)$. In relation to $\mathrm{pH}_{2}$ levels, carbon deposits were highest at low $\mathrm{pH}_{2}$ (e.g., $3.6 \mathrm{mg} \mathrm{g}_{\text {carbon feedstock }}$ ${ }^{-1}$ at $0.5 \mathrm{~atm} \mathrm{pH}_{2}$, Table 3 ). Solvent extraction of the solid carbon phases yielded no detectable PAHs ( $<1 \mathrm{ng}$ per $10 \mathrm{~cm}$ of tube), but deposits in the reactor have been shown to influence PAH formation during subsequent uses $^{58}$. In the absence of sorptive surfaces upstream (e.g., in a tube furnace), the large PAHs and solid phases could impinge on the reactive catalyst and potentially interfere with the reaction dynamics and resultant product purity $^{31}$. These competing processes could be eliminated with selective delivery of critical CNT precursors, rather than relying on thermal generation to provide a subset of necessary reactants in a complex mixture of chemicals, some of which present ecological and occupational concerns. 
Table 2. Carbonaceous deposits formed in the quartz pre-heater tube.

\begin{tabular}{cc}
\hline $\begin{array}{c}\text { Pre-heater } \\
\text { current }(\mathrm{A})\end{array}$ & $\begin{array}{c}\text { Mass of solid C deposited } \\
(\mathrm{mg} \text { C deposited } \\
\left(\mathrm{g} \mathrm{C} \mathrm{feedstock}^{-1}\right)^{\mathrm{a}}\end{array}$ \\
\hline 1.75 & $0.22 \pm 0.02$ \\
1.86 & $1.9 \pm 0.2$ \\
1.93 & $4.0 \pm 0.4$ \\
2.00 & $2.6 \pm 0.3$ \\
\hline
\end{tabular}

${ }^{a}$ Black, carbonaceous material that was not soluble in dichloromethane/methanol (90:10 mixture, at high pressure and temperature) was formed during the thermal pre-treatment of ethylene. The partial pressure of hydrogen in these experiments was 0.8 atm. Preheater current was related to temperature approximately as follows: 1.75, 1.86, 1.93, 2.0 $\mathrm{A}=860,920,970,1040^{\circ} \mathrm{C}$, respectively.

Table 3. Carbonaceous deposits formed in the quartz pre-heater as a function of hydrogen partial pressure.

\begin{tabular}{cc}
\hline $\begin{array}{c}\text { Partial } \\
\text { pressure } \mathrm{H}_{2} \\
(\mathrm{~atm})\end{array}$ & $\begin{array}{c}\text { Mass of soot deposited } \\
(\mathrm{mg} \mathrm{C} \text { deposited } \\
\left(\mathrm{g} \mathrm{C} \mathrm{feedstock}^{-1}\right)^{\mathrm{a}}\end{array}$ \\
\hline 0.0 & $3.2 \pm 0.3$ \\
0.5 & $3.6 \pm 0.4$ \\
0.8 & $1.9 \pm 0.2$ \\
\hline
\end{tabular}

${ }^{a}$ Black, carbonaceous material that was not soluble in dichloromethane/methanol (90:10 mixture, at high pressure and temperature) was formed during the thermal pre-treatment of ethylene. Soot deposition was minimized at the highest partial pressure of hydrogen. The pre-heater current of these experiments was $1.86 \mathrm{~A}\left(920^{\circ} \mathrm{C}\right)$. 


\section{Additional carbonaceous materials in the effluent contribute to smog}

formation. Many $(>25)$ other carbonaceous side products were formed during the CVD synthesis (Figures 6 and 7). Comprehensive two-dimensional gas chromatography ( $\mathrm{GC} \times \mathrm{GC})$ with time of flight mass spectrometry (TOF-MS) enabled the identification of the most abundant compounds, which included styrene, indene, and indane. Substituted benzenes, indenes, and naphthalenes were also formed during the CVD synthesis of VAMWCNTs. In general, abundances of these side products increased with increasing temperature (see Supporting information, quantification by $\mathrm{GC} \times \mathrm{GC}$ - flame ionization detection (FID)). While no concentrations exceeded occupational health guidelines, all of these compounds can contribute to smog formation, and must be quantified with photoactive VOC fraction of the effluent. 


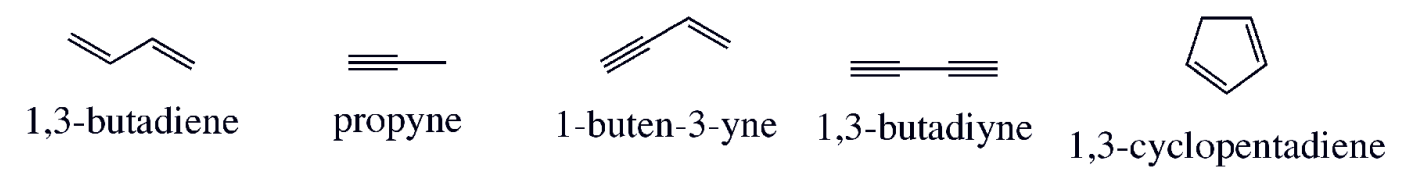<smiles></smiles><smiles>C1=Cc2cccc3cccc1c23</smiles><smiles>c1cc2c3c(cccc3c1)CC2</smiles>

naphthalene acenaphthylene

acenaphthene<smiles>c1ccc2c(c1)Cc1ccccc1-2</smiles>

fluorene<smiles>c1ccc2c(c1)ccc1ccccc12</smiles>

phenanthrene<smiles>c1ccc2cc3ccccc3cc2c1</smiles>

anthracene<smiles>c1ccc2c(c1)-c1cccc3cccc-2c13</smiles>

fluoranthene<smiles>c1cc2ccc3cccc4ccc(c1)c2c34</smiles>

pyrene<smiles>C#Cc1ccccc1</smiles>

phenylethyne<smiles>C=Cc1ccccc1</smiles>

styrene
3<smiles>C=Cc1ccccc1C</smiles>

o-methyl styrene<smiles>CCCc1ccccc1</smiles>

propylbenzene<smiles>C=Cc1cccc(C)c1</smiles>

m-methyl styrene cyclooctatetrene<smiles>C1=CC=CC=CC=C1</smiles><smiles>CC(C)c1ccccc1</smiles>

1-methylethyl benzene<smiles>C=Cc1cccc(C=C)c1</smiles><smiles>C=CCc1ccccc1</smiles><smiles>CC=Cc1ccccc1</smiles>

10<smiles>c1ccc2c(c1)CCC2</smiles>

2-propenyl benzene

1-propenyl benzene

indane<smiles>C=Cc1ccc(C=C)cc1</smiles><smiles>C#C/C=C/c1ccccc1</smiles>

1,4-diethenyl benzene 4-phenylbut-3-ene-1-yne

18<smiles>C1=Cc2ccccc2CC1</smiles>

1.2-dihydronaphthalene
20-24<smiles>Cc1ccc2ccccc2c1</smiles>

1-methyl $1 \mathrm{H}$-indene<smiles>CC1C=Cc2ccccc21</smiles>

25<smiles>Cc1cccc2ccccc12</smiles><smiles>Cc1ccc2ccccc2c1</smiles>

2-methyl

$$
\mathrm{C}_{2} \text {-substituted indenes 1-methyl naphthalene naphthalene }
$$

Figure 8. VOC and PAH structures of compounds identified in CVD effluent during VAMWCNT synthesis. Bold-faced numbers refer to peak numbers that appear in Figure 9. Compounds 20 and 21 are $\mathrm{C}_{2}$-indene isomers with no double bond in the alkyl substituent(s), whereas compounds 22-24 are $\mathrm{C}_{2}$-indene isomers with one double bond in the alkyl chain(s). There was insufficient mass spectral information to identify compound 14, and no structure is shown. 


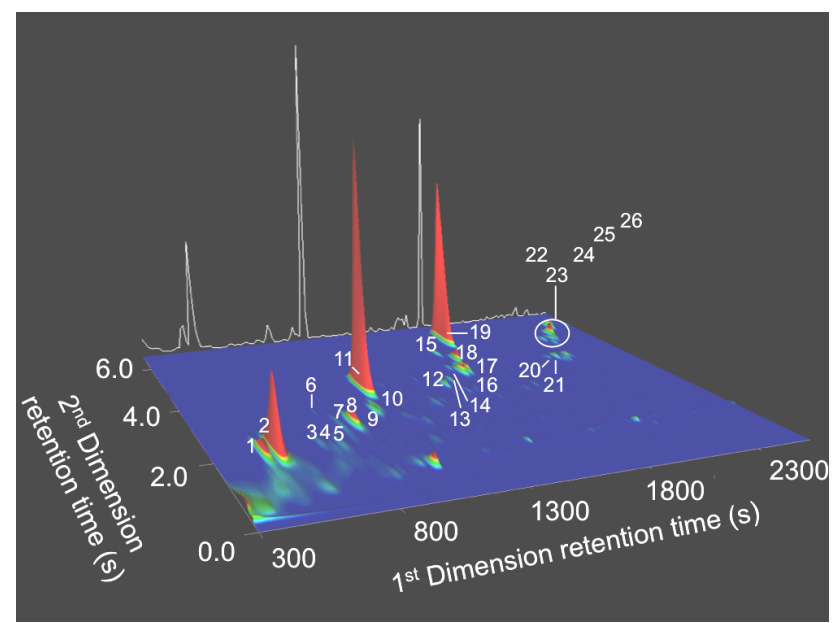

Figure 9. Comprehensive two-dimensional gas chromatogram of hydrophobic materials collected from the CVD effluent. Numbers correspond to compound identities noted in Figure 8. Here, the FID trace is shown as a three-dimensional plot and peak volume corresponds to compound abundance. A mathematically reconstructed one-dimensional trace is shown in white in the background. Compounds that were more abundant (by a factor of 3 or more) than in a blank PUF are labeled with a number. There was a series of compounds in the foreground that are unlabelled, as they are present in the PUF blank. These were alkanes (the most abundant is $n-\mathrm{C}_{11}$ ) and xylenes.

Extension to alternative CNT production methods. Within a single synthetic approach, small variations in system parameters (e.g., temperature, $\mathrm{H} / \mathrm{C}$ ratio in feedstock mixture) can cause dramatic differences in the abundance and identity of the resultant byproducts. Thus, we expect that there will be substantial differences among the various methods to produce CNTs, such as arc discharge, laser ablation, and CVD using alternate starting materials (e.g., $\mathrm{CO}$ or $\left.\mathrm{CH}_{4}\right)$. Additionally, we argue that each method should be evaluated with respect to (a) energy consumption, (b) starting material use ${ }^{30,59}$, and (c) the unintended by-products formed during the synthesis. With this comprehensive comparison of environmental metrics (and simultaneous consideration of product quality), the most benign production methods can be selected at this relatively early stage.

While it would be naïve to draw inferences from a single study to any other method, it is reasonable to suggest that the high temperature thermal treatment of any gaseous hydrocarbon will lead to the production of VOCs, PAHs, and soot. Further, one 
might expect that larger or more reactive starting materials (e.g., benzene or acetylene, respectively) will produce larger $\mathrm{VOC}, \mathrm{PAH}$, and soot species for a given amount of thermal energy. Similarly, small, less reactive compounds (e.g. methane) will produce fewer by-products for a given amount of heat delivered. Indeed, CNTs produced by methane-based CVD often yield higher-purity CNTs and acetylene-based syntheses often require minimal thermal treatment of the feedstock gas (e.g., by low temperature or low thermal contact time) to avoid "over sooting". CNT production from non-hydrocarbon feedstock gas (e.g., $\mathrm{CO})$ is purported to yield very little amorphous carbon material ${ }^{60}$ and online mass spectral analysis shows only $\mathrm{CO}_{2}$ as a by-product ${ }^{61}$. Arc-discharge and laser ablation techniques, which rely on the construction of CNT structures from solid graphite, might be expected to yield more solid-phase by-products (e.g., soot), but it is certainly possible that semi-volatile and volatile contaminants could form in these highenergy environments.

Conclusions and implications for future CNT production management. We have demonstrated that thermal decomposition of reagent gases $\left(\mathrm{C}_{2} \mathrm{H}_{4} / \mathrm{H}_{2}\right)$ during CVD growth of CNTs results in the formation of numerous hazardous air pollutants, greenhouse gases, and carcinogens. While currently negligible relative to other emissions, their environmental signal may increase to unacceptable levels as the industry continues to grow. At this early stage in the growth of the CNT industry, it is possible to circumvent the potential problems these may cause in several ways: (1) implement adequate filtration technologies (e.g., paraffin bubblers with short contact times may not be adequate) for effluent treatment, (2) recycle effluents for reuse in subsequent CNT syntheses by removing trace contaminants and soot, recollecting critical feedstock components, and generating energy onsite from appropriate materials (e.g., $\left.\mathrm{H}_{2}\right)$, or (3) reduce the formation of compounds of concern through a modified synthetic approach. The former two approaches require little-to-no technological development, simply commitment from the responsible parties. The latter approach requires iterative collaboration between materials scientists and environmental chemists (Figure 1), and this data set enables the first steps toward improved synthetic techniques. 
Additionally, armed with a thorough description of the thermally derived products, we can begin to consider which compounds are most active as CNT precursors. By selecting the appropriate gaseous component(s) as starting materials, instead of relying on thermal generation of the compounds, one could substantially improve resource use during VA-MWCNT synthesis. In particular, (1) reaction efficiency may improve (in our studies, carbon conversion efficiencies did not exceed $0.2 \%$ !), (2) chemical and energetic costs will be greatly reduced, (3) amorphous carbon formation will decrease, thereby increasing the purity and performance of the product ${ }^{31}$, (4) formation of undesirable compounds of environmental concern will be reduced, and (5) future clean-up and public health reparations will be avoided.

\section{EXPERIMENTAL METHODS}

CNT synthesis. In order to assess the effect of thermal pretreatment of reaction gases on CNT growth, we utilized a custom-built CVD reactor ${ }^{31,32}$, in which substrate (supporting the catalyst) and gas temperature were controlled independently. Pre-mixed gases were heated in a quartz pre-heater tube ( 4 x $300 \mathrm{~mm}$ (inner diameter x length), 75 $\mathrm{mm}$ heated zone) and delivered to a quartz reactor tube $(4.8 \times 22.9 \mathrm{~cm})$. Inside the reactor tube, VA-MWCNT thin films were grown on electron-beam deposited Fe $(1.2$ $\mathrm{nm})$ with an $\mathrm{Al}_{2} \mathrm{O}_{3}(10 \mathrm{~nm})$ under layer on a $\mathrm{Si}(600 \mathrm{um})$ support. The catalyst substrate temperature was regulated by a localized, resistively heated silicon platform, thereby minimizing homogenous gas phase reactions in the reactor tube. The temperature of the pre-heater tube was determined by a thermocouple placed in the center of the resistively heated coils on the outside of the quartz tube. The transit time of the gas across the heated zone was roughly $130 \mathrm{~ms}$, and heat transfer estimates suggest that roughly $30 \mathrm{~ms}$ were needed to bring the gas to the target temperature. The temperature of the silicon platform was measured using an infrared sensor mounted below the reactor tube, and the VA-MWCNT film height was measured in real-time using a laser displacement sensor mounted above the chamber. 
In order to eliminate effects of tube age ${ }^{58}$, pre-heater tubes were pre-combusted and changed for each experiment. Reactant gases were introduced according to the following program: He at $2000 \mathrm{sccm}$ for $8 \mathrm{~min}$ (where the pre-heater is turned on after 5 $\min$ ), $\mathrm{He}$ and $\mathrm{H}_{2}$ at 70 and $330 \mathrm{sccm}$ for $4 \mathrm{~min}$, respectively, (where the substrate is turned on after $2 \mathrm{~min}$ ), and then $\mathrm{C}_{2} \mathrm{H}_{4}$ and $\mathrm{H}_{2}$ at 70 and $330 \mathrm{sccm}$, respectively, for the duration of the VA-CNT growth. The end of the CNT growth was designated as the time at which the height of the VA-CNT film stopped increasing.

VOC collection and analysis. Stainless-steel 300-mL gas canisters were placed downstream from the pre-heater and reactor tubes. Considering the flow rate of the gases, we note that these samples represent a 45 -second integrated signal of VOCs forming during the reaction. Gas samples were collected just after growth termination and sealed with stainless-steel ball valves.

VOCs were quantified by gas chromatography with a flame ionization detector (GC-FID) on an HP5890 II (HayeSep-Q, 80/100 mesh, 6' x 1/8” x 0.085”, 15 mL min ${ }^{-1}$ He carrier gas flow, $50^{\circ} \mathrm{C}$ for $10 \mathrm{~min}, 6^{\circ} \mathrm{C} \mathrm{min}^{-1}$ to $100^{\circ} \mathrm{C}, 8^{\circ} \mathrm{C} \mathrm{min}{ }^{-1}$ to $160^{\circ} \mathrm{C}, 12^{\circ} \mathrm{C} \mathrm{min}^{-1}$ to $230^{\circ} \mathrm{C}, 7 \mathrm{~min}$ hold) calibrated with standard gas mixtures. Gas samples were prefocused using a cryogenic $\left(\mathrm{N}_{2}(l)\right)$ trap of Porasil-C treated silica beads. Unidentified carbonaceous species were analyzed by GC-mass spectrometry (GC-MS) on an HP G1530A (DB-624, $60 \mathrm{~m}$ x $0.25 \mathrm{~mm}$ I.D. x $1.4 \mathrm{um}$ film, $32 \mathrm{~cm} \mathrm{~s}^{-1}, 45^{\circ} \mathrm{C}$ for $10 \mathrm{~min}, 12^{\circ} \mathrm{C}$ $\min ^{-1}$ to $190^{\circ} \mathrm{C}, 2 \mathrm{~min}$ hold, $6^{\circ} \mathrm{C} \min ^{-1}$ to $225^{\circ} \mathrm{C}, 1$ min hold) interfaced to an HP 5873 MSD. The $\mathrm{CO}_{2}, \mathrm{He}$, and $\mathrm{H}_{2}$ contents were quantified by $\mathrm{GC}$ interfaced to a thermal conductivity detector (HP 5890 II, MoleSieve, 80/100 mesh, 6’ x 1/8” x 0.085”, N2 carrier gas, $70^{\circ} \mathrm{C}$ isothermal).

Unfortunately, the large quantity of ethene in our samples inhibited the quantification of ethyne, as the two components could not be fully resolved by gas chromatography in our system. Using mass spectral reconstruction of selected ions, we were able to confirm the presence of ethyne in these samples (as an unresolved shoulder on a large ethene peak). Previous studies have suggested that unheated ethyne (acetylene) can add directly to a growing VA-MWCNT carbon lattice at the Fe catalyst ${ }^{49}$. 
In addition, ethyne may play a role in gas phase reactions in the pre-heater that give rise to larger carbonaceous species. For example, ethyne is able to react with a $\mathrm{C}_{1}$-compound to form propyne ${ }^{62}$, which was relatively abundant ( $36 \pm 2$ to $\left.460 \pm 50 \mathrm{ppm}\right)$ in the effluent gas.

PAH collection and analysis. PAHs were concentrated on two consecutive polyurethane foam (PUF) filters (3" length x 1" diameter). These filters were in place for the entire duration of CNT growth, and the reported PAH abundances represent an integrated signal throughout the growth period. The PUF filters were downstream of two quartz fiber filters (QFFs), which collect particulate material ( $>0.2 \mathrm{um})$.

PUFs were pre-cleaned by triplicate accelerated solvent extractions (ASE) with a 90:10 dichloromethane: methanol mixture at $100^{\circ} \mathrm{C}$ and 1000 psi for $5 \mathrm{~min}$. The same routine was used to extract PAHs from the filters. The three serial extracts were combined, concentrated by rotary evaporation, and analyzed by GC-MS (HP 6890- JEOL GCmate, HP5-MS column, 30 m x 0.25 mm I.D. x 0.25 um film). Sample recovery was assessed using internal standards ( $d_{10}$-acenaphthalene, $m$-terphenyl, and $d_{12}$-perylene) and ranged from $75 \pm 1 \%$ for low molecular weight PAHs (128 to $154 \mathrm{amu}$ ) to greater than $90 \pm 1 \%$ for higher molecular weight PAHs (> $166 \mathrm{amu})$. Injection efficiency was monitored using a second suite of deuterated standards ( $d_{10}$-phenanthrene, $d_{10}$-pyrene, $d_{12}$-chrysene). PUF extracts were also analyzed by comprehensive two-dimensional gas chromatography (GC×GC; LECO Pegasus IV- Agilent 6890N; column 1: RTX-1 (15 m x $0.25 \mathrm{~mm}$ I.D. x $0.25 \mathrm{um}$ film); column 2: SGE BPE-50 (0.9 m x $0.10 \mathrm{~mm}$ I.D. x $0.10 \mathrm{um}$ film)).

Pre-heater tube deposits and adsorbed PAHs. Each pre-heater tube was sectioned into an even number of segments. One set of these was used to quantify the carbonaceous deposits, and the other set was used to identify sorbed compounds. Sorbed PAHs were extracted by ASE and analyzed by GC-MS (as described earlier). Carbonaceous deposits were quantified by closed-tube-combustion and vacuum line quantification $^{63}$. Briefly, segments of the tube were sealed in $9 \mathrm{~mm}$ quartz tubes with $\sim 2$ 
g cupric oxide and combusted for $5 \mathrm{hrs}$ at $850{ }^{\circ} \mathrm{C}$. The resultant $\mathrm{CO}_{2}$ was quantified on a calibrated vacuum line.

\section{ACKNOWLEDGMENTS}

A. John Hart is thanked for his contribution to this work. The authors would like to thank the National Science Foundation's Graduate Research Fellowship Program, the Woods Hole Oceanographic Institution's Ocean Ventures Fund, Arunas and Pam Chesonis through the MIT Earth System Initiative Ignition Grant program, The Seaver Institute, and the Martin Family Society of Graduate Fellows for Sustainability. We are extremely grateful for J.S. Seewald, E. Reeves, S.P. Sylva, and R.K. Nelson for the use of laboratory equipment and invaluable technical support. We thank E.R. Meshot, G.D. Nessim, B. Wardle, A. Slocum, and C.V. Thompson for useful discussions and resources.

\section{REFERENCES}

[1] Hutchison, J.E. Greener nanoscience: A proactive approach to advancing applications and reducing implications of nanotechnology. ACS Nano. 2008, 2 (3), 395-402.

[2] Maynard, A.D.; Aitken, R.J.; Butz, T.; Colvin, V.; Donaldson, K.; Oberdörster, G.; Philbert, M.A.; Ryan, J.; Seaton, A.; Stone, V.; Tinkle, S.S.; Tran, L.; Walker, N.J.; Warheit, D.B. Safe handling of nanotechnology. Nature 2006, 444, 267-269.

[3] National Academy of Sciences News Release, "Federal research plan inadequate to shed light on health and environmental risks posed by nanomaterials." Dec 10, 2008.

[4] DuPont, DuPont ${ }^{\mathrm{TM}}$ Progress Report on PFOA Phase Out. 2008. http://www2.dupont.com/PFOA2/en_US/assets/downloads/pdf/PFOARepor tExecSummary_20080114.pdf

[5] U.S. Environmental Protection Agency. EPA Announces Substantial Decrease of PFOA. 2008.

http://yosemite.epa.gov/opa/admpress.nsf/bd4379a92ceceeac852573590040

0c27/8f9dbdd044050f71852573e50064439f!OpenDocument 
[6] DuPont, New DuPoont ${ }^{\mathrm{TM}}$ Capstone ${ }^{\mathrm{TM}}$ for Repellents and Surfactants Deliver Maximum Performance, Minimal Environmental Footprint. 2008, http://www2.dupont.com/Capstone/en_US/assets/downloads/final_press_rel ease_english_3_20_2008.pdf

[7] Schwarzenbach, R.P.; Gschwend, P.M.; Imboden, D.M. Environmental Organic Chemistry, $2^{\text {nd }}$ Edition. 2003 New York, NY. John Wiley and Sons.

[8] Pidgeon, N.; Harthorn, B.H.; Bryant, K.; Rogers-Hayden, T. Deliberating the risks of nanotechnologies for energy and health applications in the United States and United Kingdom. Nature Nanotechnology 2009, 4, 95-98.

[9] Dahl, J.A.; Maddux, B.L.S.; Hutchison, J.E. Toward greener nanosynthesis. Chem. Rev. 2007, 107, 2228-2269.

[10] Anastas, P.; Warner, J. Green Chemistry: Theory and Practice. 2000 New York, NY. Oxford University Press.

[11] Plata, D.L.; Gschwend, P.M.; Reddy, C.M. Industrially synthesized singlewalled carbon nanotubes: compositional data for users, environmental risk assessments, and source apportionment. Nanotechnology 2008, 19, 185706.

[12] Gutowski, T.G.; Branham, M.S.; Dahmus, J.B.; Jones, A.J.; Thiriez, J.; Thiriez, A.; Sekulic, D.P. Thermodynamic analysis of resources used in manufacturing processes. Environ. Sci. Technol. 2009, doi: $10.1021 /$ es8016655.

[13] Linkov, I.; Satterstrom, F.K.; Steevens, J.; Ferguson, E.; Pleus, R.C. Multicriteria decision analysis and environmental risk assessment for nanomaterials. J. Nanoparticle Research 2007, 9 (4), 543-554.

[14] See, C.H.; Harris, A.T. A review of carbon nanotube synthesis via fluidizedbed chemical vapor deposition. Ind. Eng. Chem. Res. 2007, 46, 997-1012.

[15] Lamouroux, E.; Serp, P.; Kalck, P. Catalytic routes towards single wall carbon nanotubes. Catalysis Rev. 2007, 49, 341-405.

[16] Towell, G.D.; Martin, J.J. Kinetic data from nonisothermal experiments: Thermal decomposition of ethane, ethylene, and acetylene. A.I.Ch. E. Journal 1961, 7 (4), 693-698.

[17] Dean, A.M. Detailed kinetic modeling of autocatalysis in methane pyrolysis. J. Phys. Chem. 1990, 94, 1432-1439. 
[18] Bohm, H.; Jander, H. PAH formation in acetylene-benzene pyrolysis. Phys. Chem. Chem Phys. 1999, 1, 3775-3781.

[19] Frenklach, M.F.; Clary, D.W.; Gardiner, Jr., W.C.; Stein, S.E. Effect of fuel structure on pathways to soot. Twenty-first symposium (International) on Combustion, The Combustion Institute, 1986, 1067-1076.

[20] National Research Council. Vapor-phase organic pollutants: volatile hydrocarbons and oxidation products In the series Medical and Biological Effects of Environmental Pollutants. 1976 Washington, D.C. National Academy of Sciences.

[21] National Research Council. Airborne particles In the series Medical and Biological Effects of Environmental Pollutants. 1979 Baltimore, MD. University Park Press, National Academy of Sciences.

[22] U.S. Environmental Protection Agency, 40 CRF Pars 50 and 58, National Ambient Air Quality Standards for Ozone, Final Rule, Federal Register, 2008. http://www.epa.gov/fedrgstr/EPA-AIR/2008/March/Day27/a5645.pdf

[23] U.S. Environmental Protection Agency, Counties with Monitors Violating the 1997 8-hour Ozone standard of 0.08 parts per million (based on 2004-2006 Air Quality Data), 2008. http://www.epa.gov/air/ozonepollution/pdfs/20032005_Design_Value_Color.pdf, http://www.epa.gov/air/ozonepollution/pdfs/2008_03_monitors_violating_1 997.pdf

[24] Ago, H.; Uehara, N.; Yoshihara, N.; Tsuji, M.; Yumura, M.; Tomonaga, N.; Setoguchi, T. Gas analysis of the CVD process for high yield growth of carbon nanotubes over metal-supported catalysts. Carbon 2006, 44, 29122918.

[25] Tian, Y.; Hu, Z.; Yang, Y.; Chen, X.; Ji, W.; Chen, Yi. Thermal analysis-mass spectroscopy coupling as a powerful technique to study the growth of carbon nanotubes from benzene. Chem. Phys. Lett. 2004, 388, 259-262.

[26] Kim, S-M.; Zhang, Y.; Teo, K.B.K.; Bell, M.S.; Gangloff, L.; Wang, X.L.; Milne, W.I.; Wu, J.; Jiao, J.; Lee, S-B. SWCNT growth on Al/Fe/Mo investigated by in situ mass spectroscopy. Nanotechnology 2007, 18, 185709(6). 
[27] Schmitt, T.C.; Biris, A.S.; Miller, D.W.; Biris, A.R.; Lupu, D.; Trigwell, S.; Rahman, Z.U. Analysis of effluent gases during the CCVD growth of multiwall carbon nanotubes from acetylene. Carbon 2006, 44, 2032-2038.

[28] Eklund, P.C.; Ajayan, P.M.; Blackmon, R.; Hart, A.J.; Kong, J.; Pradhan, B.; Rao, A.; Rinzler, A.G. International Assessment of Research and Development on Carbon Nanotubes: Manufacturing and Applications, World Technology Evaluation Center Report, 2008, http://www.wtec.org/cnm.

[29] Thayer, A.M. Carbon nanotubes by the metric ton. Chemical \& Engineering News 2007, 85 (46), 29-35.

[30] Healy, M.L.; Dahlben, L.J.; Isaacs, J.A. Environmental assessment of singlewalled carbon nanotube processes. J. Industrial Ecology 2008, 12 (3), 376393.

[31] Meshot, E.R.; Tawfick, S.; Plata, D.L.; Verploegen, E.; Hart, A.J. Decoupled thermal treatment of precursor and catalyst enables control of diameter, structural quality, and growth kinetics of vertically aligned carbon nanotubes. Submitted to ACS Nano.

[32] Hart, A.J.; van Laake, L.C.; Slocum, A.H. Desktop growth of carbon nanotube monoliths with in situ optical imaging. Small 2007, 3 (5), 772-777.

[33] van Laake, L.C.; Hart, A.J.; Slocum, A.H. A suspended heated silicon platform for rapid thermal control of surface reactions with application to carbon nanotube synthesis. Review of Scientific Instruments 2007, 78, 083901.

[34] Choi, K.Y.; Ray, W.H. Polymerization of olefins through heterogeneous catalysis. II. Kinetics of gas phase propylene polymerization with ZieglerNatta catalysts. J. Appl. Polymer Sci. 1985, 30, 1065-1081.

[35] Jaber, I.A.; Ray, W.H. Polymerization of olefins through heterogeneous catalysis. XIV. The influence of temperature in the solution copolymerization of ethylene. J. Appl. Polymer Sci. 1993, 50, 201-215.

[36] Jaber, I.A.; Ray, W.H. Polymerization of olefins through heterogeneous catalysis. XIV. The influence of pressure in the solution copolymerization of ethylene. J. Appl. Polymer Sci. 1993, 50, 217-231.

[37] Gutowski, T.G.; Branham, M. Department of Mechanical Engineering, Massachusetts Institute of Technology, Personal Communication, 2008. 
[38] Okita, A.; Suda, Y.; Oda, A.; Nakamura, J.; Ozeki, A.; Bhattacharyya, K.; Sugawara, H.; Sakai, Y. Effects of hydrogen on carbon nanotube formation in $\mathrm{CH}_{4} / \mathrm{H} 2$ plasmas. Carbon 2007, 45, 1518-1526.

[39] Jungen, A.; Stampfer, C.; Durrer, L.; Helbling, T.; and Hierold, C. Amorphous carbon contamination monitoring and process optimization for singlewalled carbon nanotube integration. Nanotechnology 2007, 18, 075603.

[40] Stein, S.E.; Fahr, A. High-temperature stabilities of hydrocarbons. J. Phys. Chem, 1985, 89 (17), 3714-3725.

[41] National Institute for Occupational Safety and Health. NIOSH Pocket Guide to Chemical Hazards. 2005. Washington, D.C. Centers for Disease Control and Prevention.

[42] Dean, K.A. A new era: Nanotube displays. Nature Photonics 2007, 1 (5), 273 275.

[43] Euromonitor International. Global Market Information Database: Consumer Electronics. 2007. London, UK: Euromonitor International Plc.

[44] Frost and Sullivan. An Assessment on the Future of Carbon NanotubesStrategic Analysis of the Market and Potential. 2006. New York, NY: Frost and Sullivan Ltd.

[45] U.S. Environmental Protection Agency. National Air Quality and Emissions Trends Report: 2003 Special Studies Edition. 2003. Research Triangle Park, N.C. pp. 9-47. (http://www.epa.gov/air/airtrends/aqtrnd03/)

[46] U.S. Environmental Protection Agency. Methane Sources and Emissions. 2003. http://www.epa.gov/methane/sources.html

[47] U.S. Environmental Protection Agency. Toxics Release Inventory. 2006. http://www.epa.gov/tri/

[48] U.S. Environmental Protection Agency. Hanna, S.; Paine, R.; Heinold, D.; Kintigh, E.; Frey, H.C.; Baker, D.; Karp, R. Uncertainties in benzene and 1,3-butadiene emissions in Houston and their effects on uncertainties in concentrations calculated by AERMOD and ISC.

[49] http://www.epa.gov/ttn/chief/conference/ei13/uncertainty/hanna.pdf. Accessed April 2008. 
[50] Eres, G.; Kinkhabwala, A.A.; Cui, H.; Geohegan, D.B.; Puretzky, A.A.; Lowndes, D.H. Molecular beam-controlled nucleation and growth of vertically aligned single-wall carbon nanotube arrays. J. Phys. Chem. B. 2005, 109 (35), 16684-16694.

[51] Chen, Y-C.; Lee, W-J.; Uang, S-N.; Lee, S-H., Tsai, P-J. Characteristics of polycyclic aromatic hydrocarbon (PAH) emissions from a $\mathrm{UH}-1 \mathrm{H}$ helicopter engine and its impact on the ambient environment. Atmospheric Environment 2006, 40, 7589-7597.

[52] Mi, H-H.; Lee, W-J.; Chem, C-B.; Yang, H-H.; Wu, S-J. Effect of fuel aromatic content on $\mathrm{PAH}$ emission from a heavy-duty diesel engine. Chemosphere 2000, 41, 1783-1790.

[53] Galarneau, E.; Makar, P.A.; Sassi, M.; Diamond, M.L. Estimation of atmospheric emissions of six semivolatile polycyclic aromatic hydrocarbons in southern Canada and the United States by use of an emissions processing system. Environ. Sci. Technol. 2007, 41, 4205-4213.

[54] Gschwend, P.M.; Hites, R.A. Fluxes of polycyclic aromatic hydrocarbons to marine and lacustrine sediments in the northeastern United States. Geochemica et Cosmochimica Acta 1981, 45 (12), 2359-2367.

[55] Youngblood, W.; Blumer, M. Polycyclic aromatic hydrocarbons. Geochemica et Cosmochimica Acta 1975, 39, 1303-1314.

[56] Budzinski, H.; Jones, I.; Bellocq, J.; Piérard, C.; Garriges, P. Evaluation of sediment contamination by polycyclic aromatic hydrocarbons in the Gironde estuary. Marine Chemistry 1997, 58, 85-97.

[57] Yunker, M. B.; MacDonald, R.W.; Vingarzan, R.; Mitchell, R.H.; Goyette, D.; Sylvesre, S. PAHs in the Fraser River basin: a critical appraisal of PAH ratios as indicators of PAH source and composition. Organic Geochemistry 2002, 33, 489-515.

[58] Richter, H.; Howard, J.B. Formation of polycyclic aromatic hydrocarbons and their growth to soot- a review of chemical reaction pathways. Progress in Energy and Combustion Science 2000, 26, 565-608.

[59] Liu, K.; Liu, P.; Jiang, K.; Fan, S. Effect of carbon deposits on the reactor wall during the growth of multi-walled carbon nanotube arrays. Carbon 2007, 45, 2379-2387. 
[60] Robichaud, C.O.; Tanzil, .D; Weilenmann, U.; Wisner, M.R. Relative risk analysis of several manufactured nanomaterials: An insurance industry context. Enviorn. Sci. Technol. 2005, 39 (22), 8685-8994.

[61] Nikolaev, P.; Bronikowski, M.J.; Bradley, R.K.; Rohmund, F.; Colbert, D.T.; Smith, K.A.; Smalley, R.E. Gas-phase catalytic growth of single-walled carbon nanotubes from carbon monoxide. Chem. Phys. Lett. 1999, 313, 9197.

[62] Resasco, D. University of Oklahoma College of Engineering. Personal communication. 2009.

[63] Diau, E.W.; Lin, M.C.; Melius, C.F. A theoretical study of the $\mathrm{CH}_{2}+\mathrm{C}_{2} \mathrm{H}_{2}$ reaction. J. Chem Phys. 1994, 101, 3923-3927.

[64] McNichol, A.P.; Osborne, E.A.; Gagnon, A.R.; Fry, B.; Jones, G.A. TIC, TOC, DIC, DOC, PIC, POC- unique aspects in the preparation of oceanographic samples for ${ }^{14} \mathrm{C}$-AMS. Nuclear Instruments and Methods in Physics Res. 1994, B92 (1-4), 162-165. 


\section{SUPPORTING INFORMATION}

\section{PAHs are retained on cool, room temperature walls of quartz reactor tube}

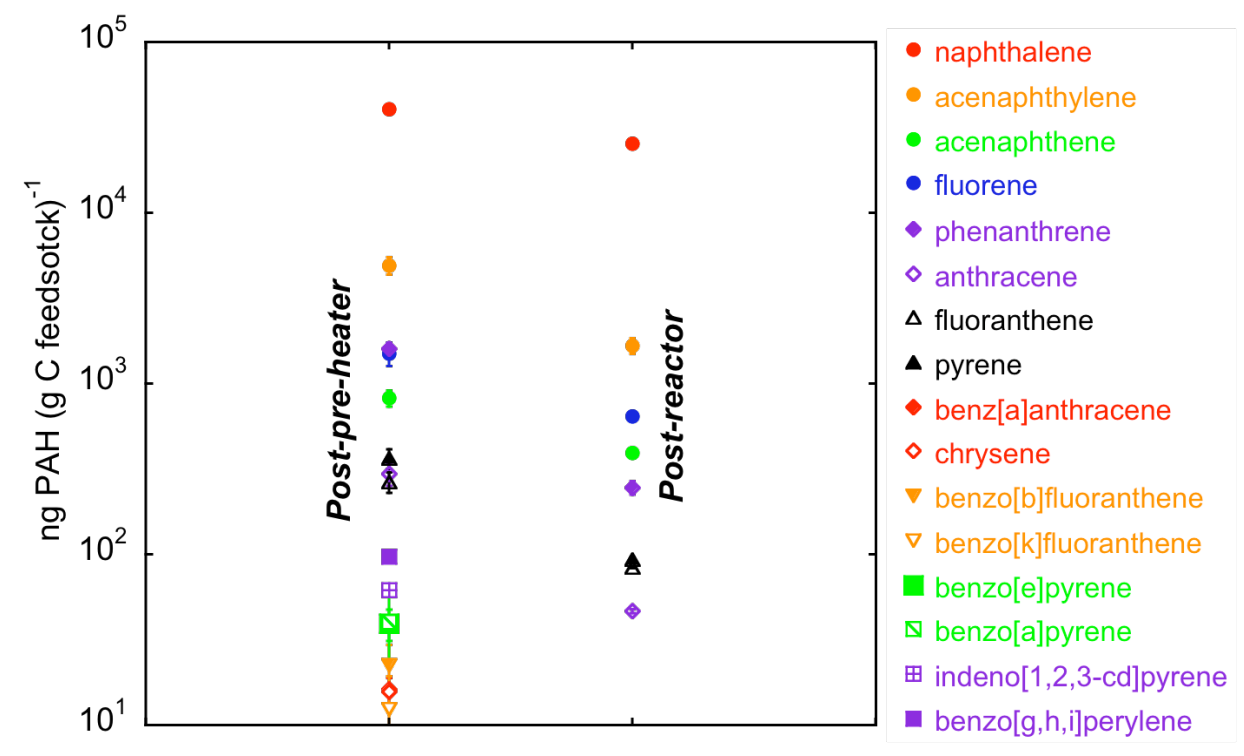

Figure S1. Polycyclic aromatic hydrogen content of gases exiting the pre-heater tube (post-pre-heater) and exiting the reactor (post-reactor). Several compounds, benzo $[b]$ fluoranthene, benzo $[k]$ fluoranthene, benzo $[e]$ pyrene, benzo $[a]$ pyrene, indeno[ $[1,2,3-c d]$ pyrene, and benzo[ $g, h, i]$ perylene, are formed in the pre-heater, but do not survive transit through the reactor tube. The total abundance of other PAHs decrease as the gas mixture travels through the reactor tube. The total mass loss of the PAHs is small compared to the mass of CNTs formed. Error bars are one standard deviation on triplicate measurements. 


\section{Scaling VA-MWCNT production to potential markets}

In both estimates, we assume that relatively short ( $\leq 100 \mathrm{um}$ ) VA-MWCNT forests will suffice. This would require roughly $1 / 10^{\text {th }}$ of the actual growth time, and we reduce our emissions by a factor of 10 to account for the truncated growth. We then calculate the average mass of ethylene that is delivered during that time, and knowing the mass of each compound per gram of ethylene (for a $1.86 \mathrm{~A}$ growth), we determine the mass of each compound that will be formed to synthesize $0.5 \mathrm{~cm}^{2}$ of VA-MWCNTs. This area is scaled up to account for the total surface area of the two applications described here:

(1) Scaling VA-MWCNT production to the size of the current liquid crystal display market

Liquid crystal display (LCD) technology accounts for a fraction ( $90 \%$ of dollar revenues in 2005) of the current flat panel display market. Annual sales of LCDs are recorded as the number of unit sales according to diagonal display size and type (e.g., television or laptop). Assuming an aspect ratio (e.g., 4:3 or 16:9) and applying the Pythagorean theorem, we can calculate the total area of each display and multiply to account for the total number of sales. Once we know the total area of LCD flat panel displays, we assume that VA-MWCNT-based displays will reach the magnitude of the LCD market. Then, the emissions generated to synthesize a given area of VA-MWCNTs are scaled to account for the total display area of the market. 
Sample calculation: Here, we assume an aspect ratio of 16:9.

Table S1. Converting total liquid crystal display unit sales to total area.

\begin{tabular}{ccc} 
Diagonal screen size $^{a}(\mathbf{i n})$ & $\mathbf{\%}_{\text {of } \text { market }^{a}}$ & Total area (cm $\left.^{(}\right)$ \\
\hline LCD Television Screens $\left(5.8 \times 10^{7}\right.$ & units) & \\
40 & 7 & $1.8 \times 10^{10}$ \\
37 & 15 & $3.3 \times 10^{10}$ \\
32 & 28 & $4.6 \times 10^{10}$ \\
30 & 50 & $7.2 \times 10^{10}$ \\
& Subtotal & $1.69 \times 10^{11}$
\end{tabular}

LCD Monitor Screens (2.35 $\times 10^{8}$ units)

27

19

17

15

$\operatorname{Other}^{b}\left(1.1 \times 10^{7}\right.$ units)

4
5

39

44

12

Subtotal

100

Subtotal
$2.4 \times 10^{10}$

$9.1 \times 10^{10}$

$8.2 \times 10^{10}$

$1.8 \times 10^{10}$

$2.15 \times 10^{11}$

$4.9 \times 10^{8}$

$4.9 \times 10^{8}$

\section{Total area of LCD displays $\quad 3.8 \times 10^{11}$}

${ }^{a}$ Data from Frost \& Sullivan using sales in 2007 (projected from base year of 2005) . ${ }^{b}$ This category includes mobile phones, PDAs, medical, public information, and automotive displays. An average diagonal screen size of 4 " was assumed.

If we assume an aspect ratio of 4:3, the total LCD area becomes $4.3 \times 10^{11} \mathrm{~cm}^{2}$. The calculations presented in the manuscript text assume a 16:9 aspect ratio. Multiplying those estimates by $1.1\left(4.3 \times 10^{11} / 3.8 \times 10^{11}\right)$ gives the emission output that would be calculated by assuming a 4:3 aspect ratio, putting bounds on the error induced by the aspect ratio assumption.

Note that these estimates are very sensitive to the VA-MWCNT area-to- effluent mass ratio. In our study, the reactor geometry was optimized to synthesize $0.5 \mathrm{~cm}^{2} \mathrm{VA}-$ MWCNTs, and novel reactor geometries may enable larger areas for a given effluent quantity. The largest area of CNTs synthesized in an array for a flat panel display was $\sim 70 \mathrm{~cm}^{2}$ (Coll et al.). As an exercise, we can make the grand (unjustified) assumption that the same effluent quantity was released during that synthesis. Then, the estimates 
presented in the manuscript text would be overestimated by a factor of $140\left(70 \mathrm{~cm}^{2} / 0.5\right.$ $\left.\mathrm{cm}^{2}\right)$.

(2) Scaling VA-MWCNT production to the size of the current household computer central processing unit (CPU) market

Annual sales of household computers were 134,826,800 in 2007 (Euromonitor

International). The average household computer contains between 1 and $3 \mathrm{~cm}^{2} \mathrm{CPU}$, and we assume a moderate value of $2 \mathrm{~cm}^{2}$ (personal communication, Intel ${ }^{\circledR}$ ). Then, we assume that the VA-MWCNT density must be at least $10 \%$ of the total surface area occupied by the forest. As-grown VA-MWCNTs in our system have a forest density of $\sim 1 \%$, and this density can be increased by squeezing the forest down to a $10^{\text {th }}$ of its original size. Thus, the necessary area of VA-MWCNTs is 10-times larger than the area of CPUs sold globally. Then, the area of CPUs in home computers is $2.7 \times 10^{8} \mathrm{~cm}^{2}$, and the area of VA-MWCNT forests needed to act as efficient TIMs is $2.7 \times \mathbf{~} \mathbf{1 0} \mathbf{c m}^{2}$. 


\section{Unique PAH isomers present in the CVD effluent}

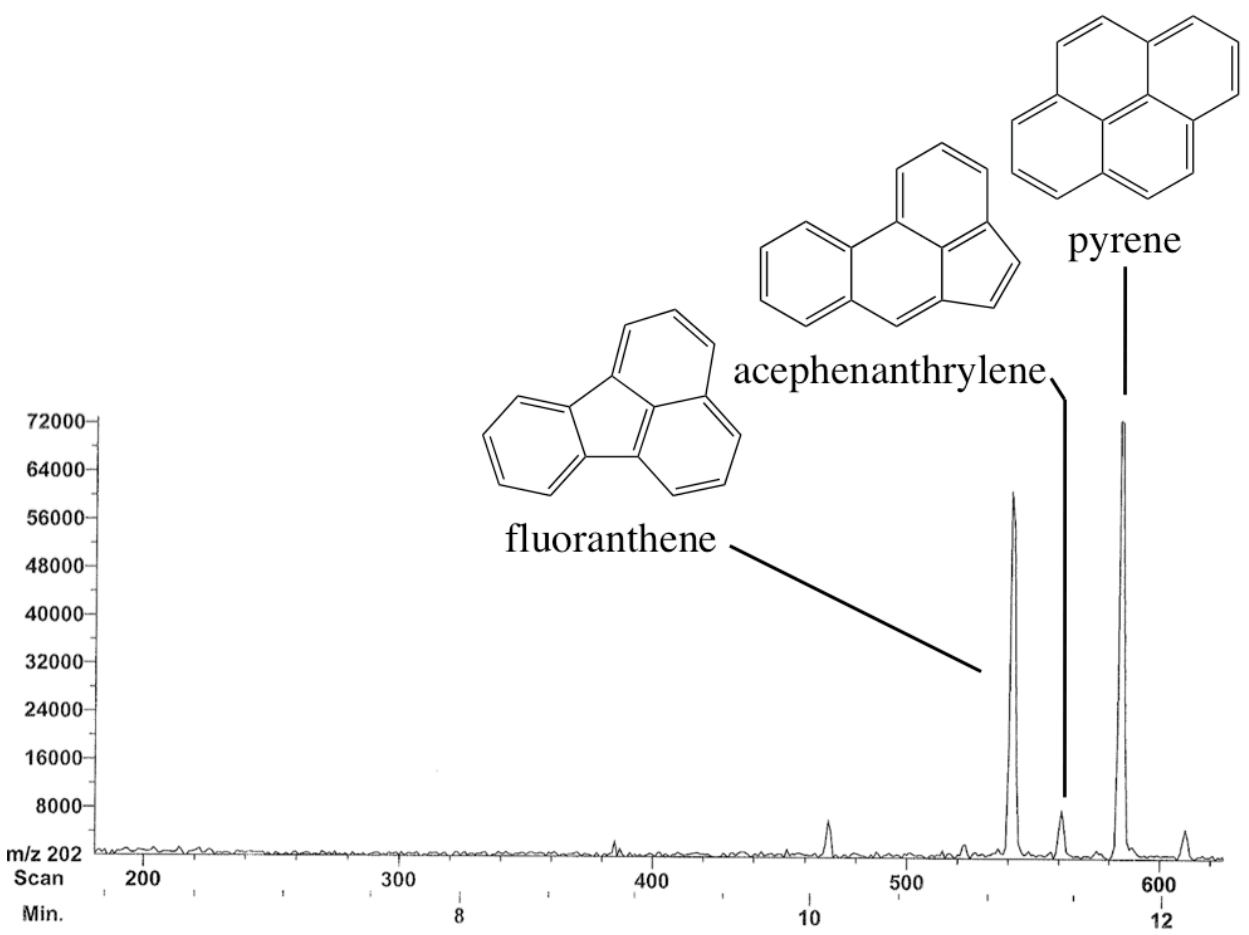

Figure S2. Extracted ion chromatogram of m/z 202 ion. Acephenanthrene was formed during CVD synthesis of MWCNTs. Pyrene and fluoranthene are commonly observed in petrogenic (fluoranthene/pyrene $<<1$ ) and pyrogenic (fluoranthene/pyrene $\geq 1$ )

emissions, but acephenanthrylene is rare (Yunker et al., Lima et al.). Acephenanthrylene quickly isomerizes to fluoranthene by thermal interconversion during pyrogenesis (Richter et al., Scott and Roeloffs). 


\section{Effects of temperature on substituted benzene formation}

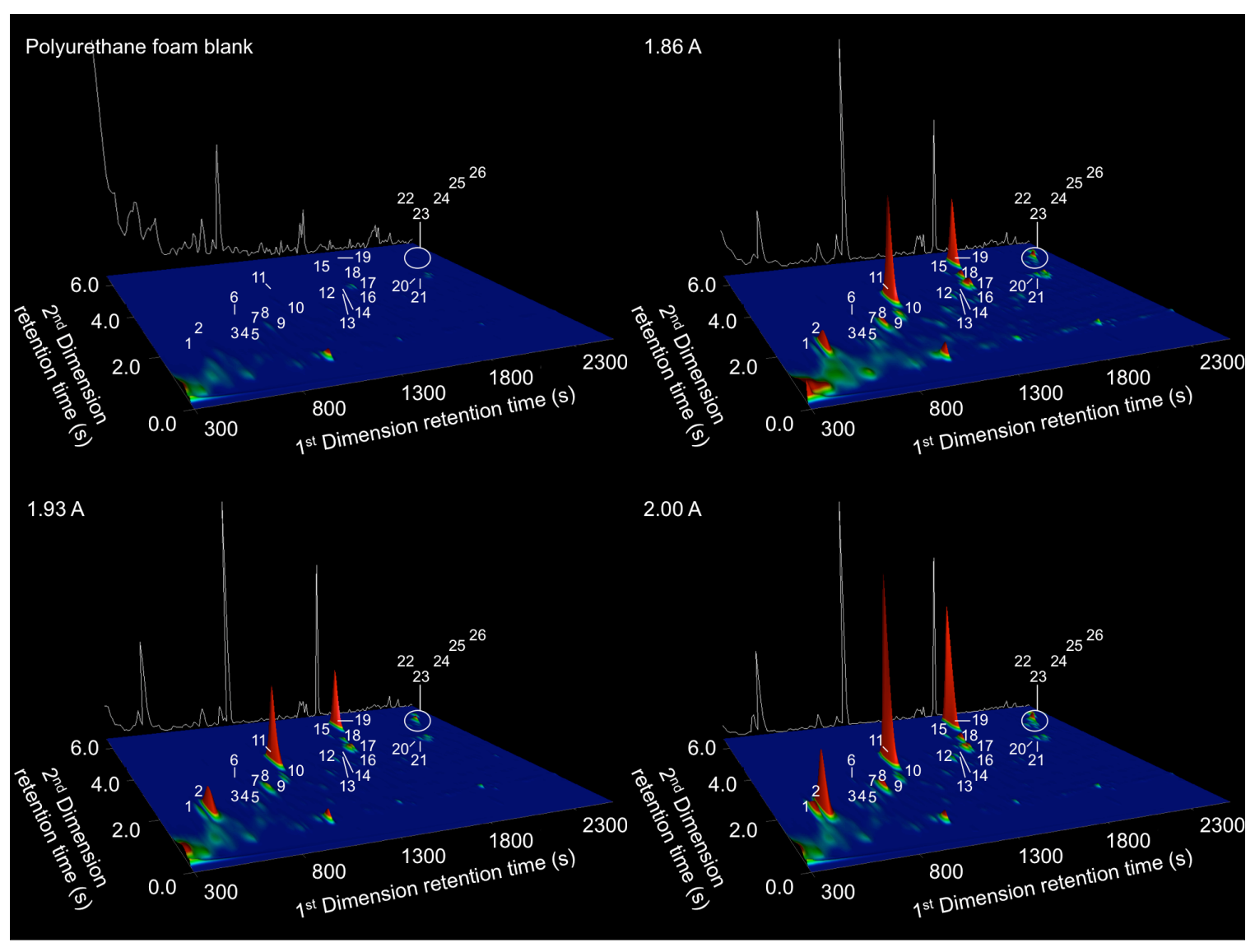

Figure S3. Comprehensive two-dimensional gas-chromatography flame-ionization detection chromatograms as a function of pre-heater current. The pre-heater current is given in the upper left hand corner of the sub-figure. Peak volume is approximately proportional to the abundance of the compound.

As pre-heater current increases, the abundance of many compounds increases. Indene is an exception, with a minimum at the 1.93 A pre-heater current. Also, there is a group of compounds that are maximized at the lowest pre-heater temperature. These unresolved peaks appear at low retention times $\left(1^{\text {st }}\right.$ and $2^{\text {nd }}$ dimensions $)$ and represent $\mathrm{C}_{2}$-substituted benzenes (xylenes). In the blank and real samples, there is a series of alkanes in the foreground of the GCxGC image. The most abundant alkane is $n-\mathrm{C}_{11}$. 


\section{Identification of compounds formed by thermal treatment of reactant gases}

Table S2. List of compounds and analytical methods used to identify the compound.



${ }^{a}$ Compound's retention time is consistent with reported boiling point or relative polarity.

${ }^{b}$ Color of checkmark indicates instrument on which the analysis was made (i.e., double checkmarks indicate that the compound was analyzed by two different instruments). 


\section{Fate of 1,3-butadiene and benzene in the atmosphere}

The primary route for human exposure to 1,3-butadiene is through air, especially in urban environments with significant automotive sources or manufacturing facilities. In the atmosphere, 1,3-butadiene is photooxidized by reaction with hydroxyl radicals, nitrate radicals, and ozone. Removal half-lives are estimated to be on the order of hours ${ }^{8}$; where daylight transformation in the troposphere is chiefly by reaction with hydroxyl radicals and nighttime removal is principally by reaction with nitrate radicals ${ }^{9}$. Physical removal from the atmosphere occurs during rain events, but the volatility of 1,3-butadiene results in return of the chemical to the atmosphere if it does not leach into the soil ${ }^{8}$.

Benzene has similar atmospheric sources and fates as 1,3-butadienes, being principally released to the atmosphere via automobile exhaust and industrial manufacturing processes. The atmospheric half-life of benzene is on the order of two weeks with hydroxyl radicals, and can be as fast as 4 hours in the presence of nitrogen oxides of sulfur dioxide, which can accelerate benzene's indirect photooxidation removal pathways ${ }^{10}$. Fairly soluble in water, benzene is removed from the atmosphere during rain events $^{10}$.

\section{REFERENCES}

[1] Frost and Sullivan. An Assessment on the Future of Carbon NanotubesStrategic Analysis of the Market and Potential. 2007 New York, NY: Frost and Sullivan Ltd.

[2] Coll, B.F.; Dean, D.A.; Howard, E.; Johnson, S.V.; Johnson, M.R.; Jaskie, J.E. Nano-emissive display technology for large-area HDTV. Journal of the Society for Information Display 2006, 14 (5), 477-485. 
[3] Euromonitor International. Global Market Information Database: Consumer Electronics. 2007. London, UK: Euromonitor International Plc.

[4] Yunker, M. B.; MacDonald, R.W.; Vingarzan, R.; Mitchell, R.H.; Goyette, D.; Sylvesre, S. PAHs in the Fraser River basin: a critical appraisal of PAH ratios as indicators of PAH source and composition. Organic Geochemistry 2002, 33, 489-515.

[5] Lima, A.L.C.; Farrington, J.W.; Reddy, C.M. Combustion-derived polycyclic aromatic hydrocarbons in the environment- A review. Environ. Forensics 2005, 6, 109-131.

[6] Richter, H.; Grieco, W.J.; Howard, J.B. Formation mechanisms of polycyclic aromatic hydrocarbons and fullerenes in premixed benzene flames. 1999, $119,1-22$.

[7] Scott, L.T.; Roelofs, N.H. Benzene ring contractions at high temperatures. Evidence from the thermal interconversions of aceanthrylene, acephenanthrylene, and fluoranthene. J. Am. Chem. Soc. 1987, 109, 54615465 .

[8] Tuazon, E.C.; Alvarado, A.; Aschman, S.M.; Atkinson, R.; Arey, J. Products of the gas-phase reactions fo 1,3-butadiene with $\mathrm{OH}$ and $\mathrm{NO}$ radicals. Environ. Sci. Technol. 1999, 33 (20), 3586-3595.

[9] Howard, P. Handbook of Environmental Fate and Exposure Data for Organic Chemicals. Vol I. Large Production and Priority Pollutants. Lewis Publishers. 1989 Chelsea, MI. USA. pp. 101-105.

[10] Howard, P. Handbook of Environmental Fate and Exposure Data for Organic Chemicals. Vol II. Solvents. Lewis Publishers. 1990 Chelsea, MI. USA. pp. 29-39. 


\title{
CHAPTER 5: \\ IMPROVED RESOURCE USE IN CARBON NANOTUBE SYNTHESIS: \\ RAPID GROWTH WITHOUT THERMAL TREATMENT OF FEEDSTOCK GASES
}

\author{
By
}

Desirée L. Plata

This work was performed in collaboration with A. John Hart and Eric R. Meshot at the University of Michigan in the Department of Mechanical Engineering. The writing and data presented in this chapter are the work of D.L. Plata, principally. 


\begin{abstract}
Carbon nanotube (CNT) production by catalytic chemical vapor deposition (CVD) is increasing globally, with current production volumes at 300 tons $\left(3 \times 10^{8} \mathrm{~g}\right)$, and recent studies suggest that environmentally concerning compounds are generated by thermal treatment of common CNT feedstock gasses $\left(\mathrm{C}_{2} \mathrm{H}_{4} / \mathrm{H}_{2}\right)$. Eliminating thermal treatment may prevent the formation of unwanted by-products (e.g., toxicants, greenhouse gases, and smog-forming compounds), reduce energetic demands, and improve overall control over the synthesis. However, heating the feedstock gas is necessary for rapid vertically aligned multi-walled CNT (VA-MWCNT) growth, presumably due to the thermal generation of critical CNT precursor molecules. Using in situ VA-MWCNT height measurements and complementary gas analysis, we identified thermally generated compounds that were correlated with VA-MWCNT formation rate (propyne and but-1en-3-yne). To demonstrate that these alkynes were responsible for rapid VA-MWCNT growth, we delivered each chemical and typical feedstock gases, without heating, directly to a locally heated metal catalyst substrate. The tested alkynes accelerated VA-MWCNT formation to rates comparable to, or greater than, those achieved via thermal treatment of the feedstock gas. Ethene and hydrogen were still required for efficient CNT formation, but their input concentrations could be reduced by 20 and $40 \%$, respectively, without sacrificing CNT growth rate. Using this new approach of unheated, alkyne-assisted VAMWCNT growth, emissions of volatile organic compounds and polycyclic aromatic hydrocarbons were reduced by more than an order of magnitude compared to traditional CVD approaches. Furthermore, the chemical studies presented here shed new light on the current understanding of CNT synthesis, suggesting that a metal-catalyzed polymerization reaction may be involved in CNT formation.
\end{abstract}




\section{INTRODUCTION}

The industrial and laboratory-scale production of carbon nanotubes (CNTs) has been increasing for the last decade, with current production volumes at 300 tons year ${ }^{-1}$ globally (ton $=10^{6} \mathrm{~g}$ ) . The preferred method for large-volume CNT production, catalytic chemical vapor deposition (CVD), is plagued by inefficiency, where no more than $3 \%$ of the introduced carbon feedstock is converted to $\mathrm{CNT}^{2}$. In some cases, the unused feedstock is recycled for subsequent nanotube growth, but in many other cases, the effluent and its associated by-products are vented to the atmosphere. These untreated materials could amount to an annual release of 10,000 tons $\left(10 \times 10^{9} \mathrm{~g}\right)$ of carbonaceous material, and this will expand to 300,000 tons year ${ }^{-1}\left(0.3 \times 10^{12} \mathrm{~g} \mathrm{year}^{-1}\right)$ within the next decade if production accelerates as predicted ${ }^{3}{ }^{3}$. Plata et al $^{4}$ recently demonstrated that the effluent from an ethene-based CVD growth contained several compounds that pose threats to the quality of the air, water and soil. These included toxics (e.g., benzene, 1,3butadiene, and aromatic hydrocarbons), greenhouse gases (e.g., methane), and compounds that contribute to smog formation and exacerbate respiratory illness. To avoid unnecessary environmental and public health damages from CNT fabrication, efforts must be made to reduce emissions and improve the efficiency of CNT synthesis. These preventative measures will not only save manufacturers immediate costs, but also prevent future loss due to production bans, environmental remediation efforts, and personal claims reparations.

Understanding the CNT formation mechanism(s) is a critical first step toward improved efficiency. In general, all CVD processes involve the introduction of a gaseous carbon precursor (e.g., $\mathrm{CO}, \mathrm{C}_{2} \mathrm{H}_{4}$, or $\mathrm{CH}_{4}$ ) to a heated (e.g., $700-1000^{\circ} \mathrm{C}$ ) reaction zone that contains a free-floating or substrate-supported metal catalyst (e.g., $\mathrm{Fe}, \mathrm{Ni}$, or Co). While the current "mechanism" is limited with respect to chemical details ${ }^{5-7}$, the CNT growth process is generally described by three stages: nucleation, elongation, and

\footnotetext{
${ }^{a}$ With a doubling rate of once every two years and assuming that the CNT mass: US \$ ratio is unchanged over the next ten years.
} 
termination. The prevailing thought is that during nucleation, the carbon-containing precursor dissociates at the metal catalyst and forms a CNT cap ${ }^{5,8}$. During elongation, carbon adds to the growing CNT by continual dissociation at, diffusion into, and precipitation from the metal catalyst (i.e., the vapor-liquid-solid (VLS) model ${ }^{9}$. This addition of single carbon units continues until termination, where mechanical stress ${ }^{10}$, catalyst encapsulation $^{11}$, and/or catalyst deactivation halts CNT growth ${ }^{12}$. As reactions at the catalyst are clearly important for CNT formation, little attention has been given to gas-phase reactions in these syntheses. However, recent work by Hart et al. ${ }^{13,14}$ has shown that there are distinct effects that arise from the independent thermal treatment of the carbonaceous feedstock and the metal catalyst. In particular, heating and cooling ("pre-heating") the feedstock gas prior to impingement on a metal catalyst is necessary for rapid growth of vertically aligned multi walled CNTs (VA-MWCNTs), enhancing the CNT formation rate by over $2000 \%$ compared to when the gas is heated only at the catalyst ${ }^{13}$.

The thermal pre-treatment step generates a suite of volatile organic compounds (VOCs) from an ethene feedstock, ${ }^{4}$ some of which may be responsible for enhanced CNT formation and others of which may diminish CNT quality ${ }^{14}$ and present environmental and occupational concern. These competing processes may be eliminated with selective delivery of critical CNT precursors, rather than relying on thermal generation to provide a subset of necessary reactants in a complex mixture of chemicals. Furthermore, avoiding the thermal treatment of the feedstock gas would remove the most energetically expensive component of the synthesis ${ }^{15,16}$ and potentially improve the carbon-to-CNT mass conversion efficiency. Here, we aim to identify compounds on the critical path to CNT formation by monitoring in situ VA-MWCNT growth rate as we deliver potentially important molecules directly to the catalyst. With these results, we can begin to cooptimize the synthetic process by (1) minimizing cost by selecting potent reagent gases that require minimal thermal treatment ${ }^{15,16},(2)$ maximizing production growth rates, and (3) minimizing unwanted side-products that deteriorate product quality (e.g., soot and polycyclic aromatic hydrocarbons (PAHs)) and threaten the health of the public and the 
environment (e.g., toxics, greenhouse gases, and compounds that promote the formation of secondary pollutants).

\section{EXPERIMENTAL METHODS}

Chemical supplies. Helium, hydrogen, ethene, methane, ethyne, and 1\% ethyne (mixed with helium) were purchased from Metro Welding (Ann Arbor, MI) or AirGas (all ultra high purity (UHP) grade). Before use and prior to introduction to a mass flow controller, pure ethyne was filtered through a Porasil-C packed column immersed in a cyrogenic solution (acetone $/ \mathrm{N}_{2}(l)$ or acetonitrile $/ \mathrm{N}_{2}(l)$, both $-39-41{ }^{\circ} \mathrm{C}$ ) to remove acetone (which is used as a stabilizer to prevent reaction in the ethyne tank). Mass spectral analysis indicated that acetone was removed to below the detection limit $(<$ ppmv) by this method. To our knowledge, this is the first CNT growth study where acetone has not been present as a co-reactant in ethyne-fed CVD experiments. The 1\% ethyne mixture did not require purification, as it was free of acetone when shipped from the manufacturer. Other gases, including 1,3-butadiene, but-1-ene-3-yne (1\% mixture in helium), propyne, ethane, and 1-butyne, were purchased from Air Liquide America Specialty Gas in high purity. Diluted mixtures of methane and ethane were prepared by flushing 300-mL stainless steel (SS) tanks with the gas of interest, pressurizing the tanks to some low pressure (e.g., 5 psi), and then further pressurizing the tank with He to approximately 250 psi. Benzene was prepared by adding over $30 \mathrm{~mL}$ of liquid benzene to a $300-\mathrm{mL}$ SS tank, flushing the tank with He (without removing the benzene), and pressurizing the tank to $250 \mathrm{psi}$. In all cases where He (UHP grade) was used to prepare test gases, it was further purified with a Porasil-C packed column immersed in $\mathrm{N}_{2}(l)$.

CNT synthesis. In order to measure in situ VA-MWCNT height during the reaction progress, we utilized a custom-built CVD reactor ${ }^{13,17}$ with a laser displacement sensor mounted above the growth chamber (see Supporting Information). In this reactor, gases are pre-mixed and introduced to a resistively heated quartz pre-heater tube $(4 \times 300$ $\mathrm{mm}$ (inner diameter $\mathrm{x}$ length)), cooled to room temperature, and then delivered to a 
quartz reactor tube $(4.8 \times 22.9 \mathrm{~cm})$. Inside the reactor tube, VA-MWCNT thin films were grown on electron-beam deposited $\mathrm{Fe}(1.2 \mathrm{~nm})$ with an $\mathrm{Al}_{2} \mathrm{O}_{3}(10 \mathrm{~nm})$ under layer on a $\mathrm{Si}$ (600 um) support. The catalyst substrate temperature was regulated by a localized, resistively heated silicon platform, thereby minimizing gas phase reactions in the growth chamber. The temperature of the silicon platform was measured using an infrared sensor mounted below the reactor tube and fixed at $725^{\circ} \mathrm{C}$ for all experiments (except where noted). The temperature of the "cool-wall" reactor was less than $70^{\circ} \mathrm{C}$ over the length of the platform $(4-5 \mathrm{~cm})$ and room temperature elsewhere, as measured by a surface-contact thermocouple place on the outside of the quartz wall. The pre-heater temperature was determined by a thermocouple placed outside of the quartz tube in the center of the resistively heated coils. The pre-heater was operated in two modes: (1) "on" at $1000{ }^{\circ} \mathrm{C}$ or (2) "off" at room temperature $\left(21^{\circ} \mathrm{C}\right)$. In both modes, reactant gases $\left(\mathrm{C}_{2} \mathrm{H}_{4} / \mathrm{H}_{2}\right)$ travelled through the pre-heater tube and connected to a three-way valve that was installed downstream of the pre-heater. This allowed the introduction of helium carrier gas and specific VOC test gases (e.g., propyne or but-1-en-3-yne) to the reactant stream mixture prior to impingement on the catalyst. The sum of these flows ( $\mathrm{He}+\mathrm{VOC}$ test gas) was constant, but the distribution varied; test gases were only introduced when the pre-heater was "off." Given the varying He delivery, we sought to remove effects associated with trace contaminants (e.g., methane or water) in the UHP grade He by purifying the carrier gas using a $\mathrm{N}_{2}(l)$ cold trap with Porasil-C treated silica beads.

While the mixture of reactant gases varied during the growth phase, the reactor flush and annealing treatments were kept constant. A typical reactant gas program included: He flush at $2000 \mathrm{sccm}$ for $8 \mathrm{~min}$ (where the pre-heater is turned on after $5 \mathrm{~min}$, if applicable), $\mathrm{He}$ and $\mathrm{H}_{2}$ at 70 and $300 \mathrm{sccm}$, respectively, for $4 \mathrm{~min}$ (where the catalyst substrate platform is turned on after $2 \mathrm{~min}$ ), and $\mathrm{C}_{2} \mathrm{H}_{4}, \mathrm{H}_{2}$, the VOC test gas, and He were introduced for the duration of the CNT growth (where the summed flow rate of these gases was always $604 \mathrm{sccm}$, except where noted).

VOC collection and analysis. VOCs were collected and analyzed as detailed in Plata et al. ${ }^{4}$ Briefly, stainless steel canisters were placed downstream of the reactor tube 
and flushed for the duration of CNT growth. Gas samples were collected just after growth termination and sealed with stainless steel ball valves. Considering the flow rate of the gas and volume of the cylinder, we note that these samples represent a 30-second integrated signal of VOCs forming during the reaction. Simultaneous measurements of VOC composition in the effluent gases were collected using an online mass spectrometer (MS, Pfeiffer OmniStar ${ }^{\mathrm{TM}}$ ) and monitoring relevant ions (m/z 2, 4, 12-18, 25-30, 32, 39$42,44-45,51-54,65-66,77-78,91)$. These real-time analyses indicated that gas composition over a 30 -second interval during the growth cycle was stable. VOCs in the stainless steel canisters were quantified by gas chromatography with a flame ionization detector and thermal conductivity detector (GD-FID-TCD with He reference gas) calibrated with standard gas mixtures. Gas samples were pre-focused using a cryogenic $\left(\mathrm{N}_{2}(l)\right)$ trap of Porasil-C treated silica beads before injection on the HayeSep Q column. Detection limits were around 0.1 ppmv. $\mathrm{He}$ and $\mathrm{H}_{2}$ were quantified using an additional GC-TCD with a $\mathrm{N}_{2}$ reference gas.

$P A H$ collection and analysis. PAHs were collected and analyzed as detailed in Plata et al. ${ }^{4}$ Briefly, PAHs were concentrated on two consecutive, pre-cleaned polyurethane foam (PUF) filters (3" length x 1" diameter). These filters were in place for the entire duration of CNT growth, and the reported PAH abundances represent an integrated signal throughout the growth period. PUFs were extracted by triplicate accelerated solvent extractions (ASE) with a 90:10 dichloromethane: methanol mixture at $100^{\circ} \mathrm{C}$ and $1000 \mathrm{psi}$ for $5 \mathrm{~min}$. Each extract was concentrated by rotary evaporation, and analyzed by GC-MS. Sample recovery was assessed using internal standards $\left(d_{10^{-}}\right.$ acenaphthalene, $m$-terphenyl, and $d_{12}$-perylene) and ranged from $75 \pm 1 \%$ for low molecular weight PAHs (128 to $154 \mathrm{amu}$ ) to greater than $90 \pm 1 \%$ for higher molecular weight PAHs ( $>166 \mathrm{amu}$ ). Detection limits were around $1 \mathrm{ng} \mathrm{g}_{\mathrm{C} \text { feedstock }}{ }^{-1}$ (an average of 0.001 parts per trillion by volume). 


\section{RESULTS AND DISCUSSION}

Accelerated CNT growth with thermal pre-treatment of feedstock gas. Using a decoupled CVD reactor, we independently controlled the temperature of VA-MWCNT metal catalyst and $\mathrm{C}_{2} \mathrm{H}_{4} / \mathrm{H}_{2}$ feedstock while monitoring the composition of gases evolved from the pre-heater, as well as the in situ VA-MWCNT forest growth rate. As the preheater temperature increased (from 690 to $1200^{\circ} \mathrm{C}$ ), the growth rate of the VA-MWCNT forest increased ${ }^{4,14}$, and we hypothesized that some subset of thermally generated compounds was responsible for the accelerated CNT formation. Indeed, there were strong linear correlations between the growth rate and the partial pressures of methane, benzene, and but-1-en-3-yne (each with a correlation coefficient of $0.99 ; n=4$, Figure 1). As the abundance of propyne increased, the growth accelerated to a point of apparent saturation, indicating that at relatively high propyne concentrations, something other than substrate concentration limited the rate of VA-MWCNT formation. The hyperbolic behavior is characteristic of catalysis reactions (see Supporting Information), suggesting that the metal catalyst may play a role in CNT formation beyond simply providing a template for highly-ordered carbon precipitation, as described by the VLS model.

The VLS model has been used to describe the probability of CNT growth from various carbon precursors, such as methane. Methane is a common CVD feedstock gas, and recent models suggest it gives effective CNT growth via decomposition on Fe catalyst surfaces $^{18}$. Benzene has been discussed as an important intermediate in CNT formation, but the alkynes propyne and but-1-en-3-yne have not been recognized as active molecules in the CNT formation pathway. Ethyne, however, has been noted for its relatively efficient conversion to CNT in molecular beam experiments (where gas phase reactions are minimized $)^{19}$. Unfortunately, in those studies, acetone was present as a trace $(<1 \%)$ component of the commercially available ethyne, and it is unclear if the enhanced growth was an effect of the ethyne itself or the oxygen-containing acetone. (Oxygen-containing compounds can enhance CNT growth ${ }^{20}$ ). Nevertheless, in homogeneous transition metal catalysis, alkynes have been shown to react with alkenes to 

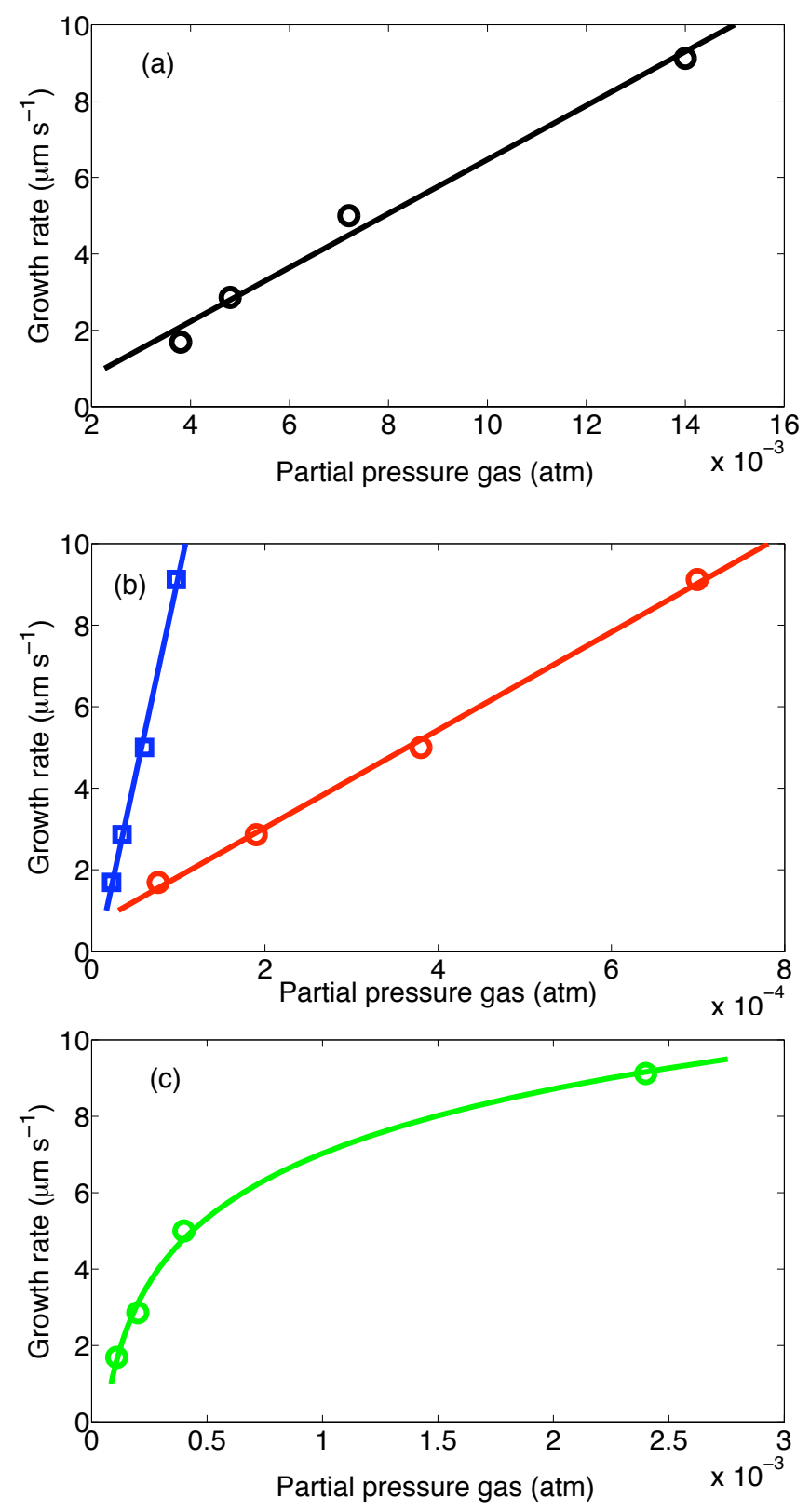

Figure 1. Thermally generated compounds correlate with increase VA-MWCNT growth rate. In all subplots, the symbols are measured data ${ }^{4}$ and the line is the best-fit curve. (a) Methane's abundance was linearly related to the growth rate $\left(\mathrm{R}^{2}=0.99, n=4\right)$. (b) Benzene (red circles) and but-1-en-3-yne (blue squares) were linearly related to the growth rate $\left(\mathrm{R}^{2}=0.99, n=4\right)$. (c) The relationship between propyne and the VAMWCNT growth rate was described by a hyperbola. In these experiments, the gas flow during growth was $\mathrm{C}_{2} \mathrm{H}_{4} / \mathrm{H}_{2} / \mathrm{He}=70 / 330 / 0 \mathrm{sccm}$ and the catalyst substrate temperature was $825^{\circ} \mathrm{C}$. 
form cyclic intermediates, which are subsequently released from the active metal ${ }^{21-24}$. Although CVD is a heterogeneous catalysis, it is possible that alkynes are playing a similar role in CNT formation.

Accelerated CNT growth without heating feedstock gases. To demonstrate that members of a chemical class, small alkynes (with $\mathrm{C}_{\mathrm{n} \leq 4}$ ), are responsible for the accelerated VA-MWCNT growth, we delivered each potential pre-cursor molecule to a heated metal catalyst without thermal treatment of the feedstock gas. To simulate the growth environment that would be generated by the pre-heater (without the convolution of the more than 40 thermally generated compounds) ${ }^{4}$, we delivered trace amounts of the test gas (e.g., < 1\% by vol), along with a supply of ethene $(18.7 \%$ by vol) and hydrogen $(51.3 \%$ by vol, balance $\mathrm{He})$, to the heated metal catalyst. When equal partial pressures $\left(9.8 \times 10^{-3} \mathrm{~atm}\right)$ of each test gas were delivered, ethyne and propyne enhanced the growth rate of CNTs to a greater extent than either 1,3-butadiene or methane (Figure $2 \mathrm{a}$; growth rates (all in $\mathrm{um} \mathrm{s}^{-1}$ ) of $3.5 \pm 0.1$ for both ethyne and propyne, and $1.1 \pm 0.1$ and $0.7 \pm 0.1$ for 1,3-butadiene and methane, respectively). (Note that mass spectral analysis showed no acetone in our cryogenically purified ethyne). The addition of ethane, an abundant product of the thermal treatment of ethene ${ }^{4}$, did not accelerate CNT growth beyond that observed without any additional test gas (the "pre-heater off") case. A third alkyne was tested, but at a slightly lower partial pressure $\left(3.0 \times 10^{-3} \mathrm{~atm}\right)$, due to concentration limitations on but-1-en-3-yne (which is unstable at high concentrations). In spite of over a factor-of-3 dilution compared to ethyne and propyne, the CNT growth rate of but-1-en-3-yne was only 1.3 times slower $\left(2.7 \pm 0.1 \mathrm{um} \mathrm{s}^{-1}\right.$ vs. $3.5 \pm 0.1 \mathrm{um} \mathrm{s}^{-1}$ ). Thus, if these concentrations are within the linear response range of but-1-en-3-yne's effect on growth, then but-1-en-3-yne could be more active than both propyne and ethyne.

We hypothesized that the additional activity of but-1-en-3-yne might arise from the double bond at the head of the molecule. To probe the effect of this functional group, we explored the accelerating effects of 1-butyne (which lacks a double bond). At the same partial pressures (3.3 $\left.\times 10^{-4} \mathrm{~atm}\right), 1$-butyne accelerated the growth of CNTs to a 
lesser extent than but-1-en-3-yne (Figure $2 b$ ). This suggests that the alkene group has a role in promoting CNT formation beyond the effects of the alkyne alone, and potential mechanisms will be discussed in light of further results (that appear later). For completeness, benzene, which was correlated to VA-MWCNT growth rate, was delivered to the catalyst as a test gas. There was limited acceleration in CNT formation at this low abundance of benzene (vapor pressure limits prevent the use of higher concentrations), but $3.3 \times 10^{-4} \mathrm{~atm}$ is within the range expected from thermal generation. Similarly, relevant concentrations of methane did not promote CNT formation rates. Thus, correlation with rate does not necessarily indicate that benzene or methane was acting to accelerate VA-MWCNT growth at the catalyst, as they may simply have been synthesized in sequence from another critical component (e.g., benzene formation propyne-derived radicals ${ }^{25}$ ).

It was possible that the perceived inactivity of methane could be due to its low carbon content (per mol gas) as compared to longer alkenes and alkynes. To ensure that the observed rate enhancements were not merely the result of differences in content, we normalized the delivery of each test precursor to a constant mass $\left(5.5 \pm 0.4 \mathrm{ug} \mathrm{C} \mathrm{sccm}{ }^{-1}\right.$, where sccm is a standard cubic centimeter per minute). On a mass basis, but-1-en-3-yne exhibited the greatest rate acceleration, followed by ethyne, propyne, 1,3-butadiene, and methane (Figure 2c). Thus, the accelerating effects of alkynes on CNT growth are not simply due to their relative carbon contents, but are intimately related to their structures.

Ultimately, it is clear that rapid CNT growth can be achieved without heating feedstock gas, which will reduce energy requirements of industrial scale CVD synthesis and likely limit the formation of unintended by-products (e.g., toxicants and greenhouse gases). 

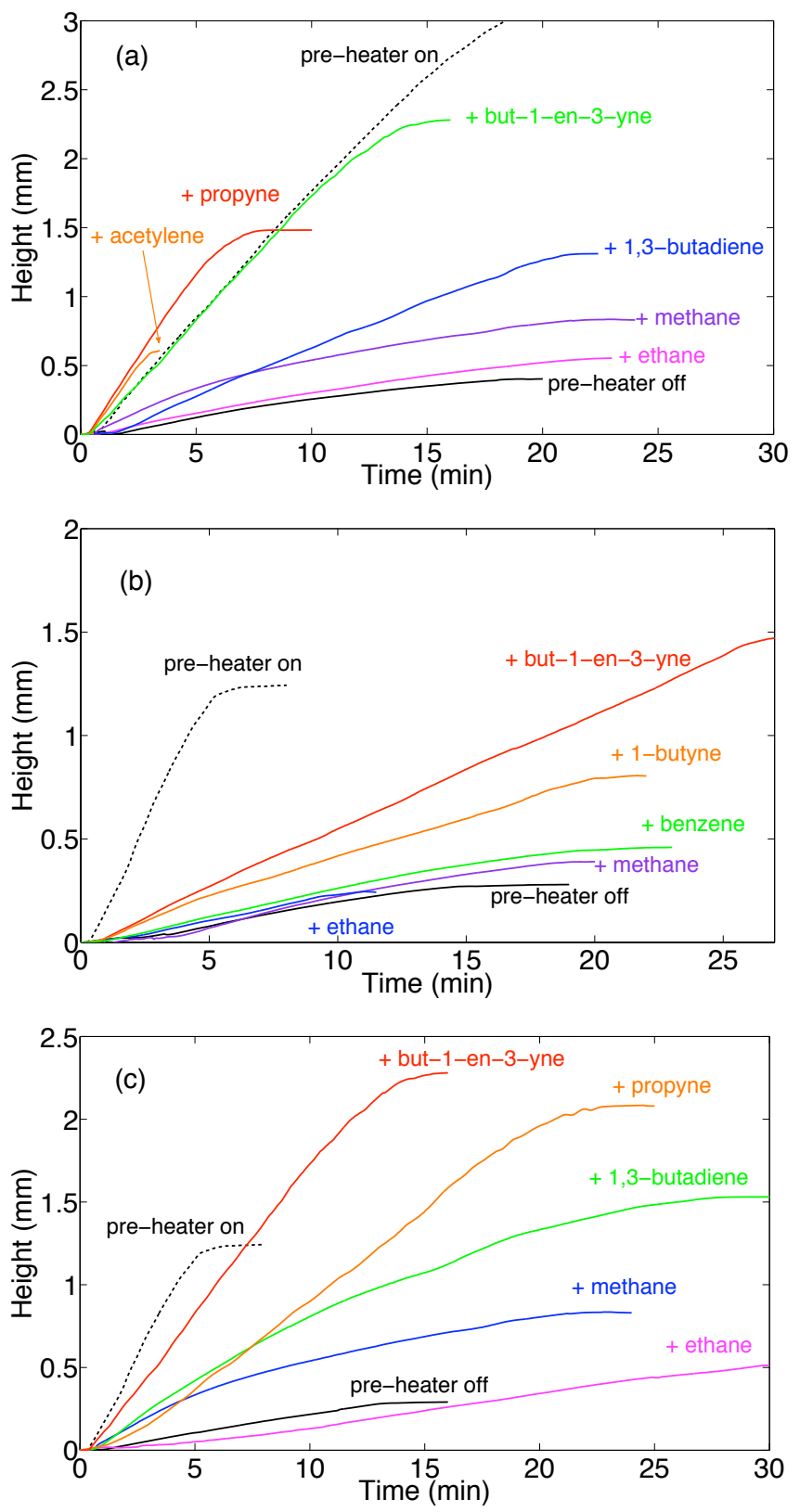

Figure 2. Effects of chemical structure on VA-MWCNT growth. In all experiments, standard growth gasses $\left(\mathrm{C}_{2} \mathrm{H}_{4} / \mathrm{H}_{2}=120 / 310 \mathrm{sccm}\right)$ were delivered without pre-heating in addition to either (1) no test gas ("pre-heater off") or (2) a test gas (e.g., propyne or methane). To provide a reference for typical growth conditions, a "pre-heater on" case (where only $\mathrm{C}_{2} \mathrm{H}_{4}$ and $\mathrm{H}_{2}$ are delivered) is also shown. (a) Test gases were delivered at equal partial pressures $\left(9.8 \times 10^{-3} \mathrm{~atm}\right)$, except for but-1-en-3-yne, which was more dilute $\left(3.0 \times 10^{-3} \mathrm{~atm}\right)$. All alkynes significantly accelerated growth compared to when they were not delivered (pre-heater off case). Test gases were then delivered at (b) lower partial pressures $\left(3.3 \times 10^{-4} \mathrm{~atm}\right)$ or $(\mathrm{c})$ equal masses $\left(5.5 \pm 0.4 \mathrm{ug} \mathrm{C} \mathrm{sccm}^{-1}\right)$. 


\section{Reduced by-product formation without sacrificing CNT growth. Although}

feedstock gases were not heated prior to impingement on the metal catalyst, they were subject to local heating in the proximity of the resistively heated catalyst substrate platform. Thus, gas phase rearrangements in the reactor tube could result in the formation of unintended side products. In the typical CVD approach, with the pre-heater on, many volatile organic compounds are generated from the thermal treatment of ethene and hydrogen (Figure 3), including methane (a potent greenhouse gas), benzene, and 1,3butadiene (hazardous air pollutants regulated by the EPA). Omitting pre-heating drastically reduces the abundance of all VOCs (except ethane) by more than an order of magnitude (see Supporting Information for reduction factors). Several compounds, benzene, 1,3-butadiyne, cyclopentadiene, pentene, and pentane, were not formed in detectable quantities ( $>0.1 \mathrm{ppmv}$ ) when the pre-heater was off. Thus, CNT fabrication techniques that limit the thermal treatment of the feedstock gas offer substantial reductions in unnecessary emissions. However, neglecting to pre-heat requires the addition of an alkyne to achieve comparable CNT growth rates and heights. The addition of propyne and but-1-en-3-yne did increase the VOC content of the effluent gas, but not above levels produced by thermal treatment of the feedstock. Compared to pre-heating, methane formation was reduced by about a factor of 30 during alkyne-assisted CNT growth; 1,3-butadiene formation was reduced by more than a factor of 60; and benzene formation was reduced below the detection limit $(<0.1 \mathrm{ppmv})$. Benzene was only formed when 1,3-butadiene was added to the unheated feedstock gases, and VOCs were highest compared to all other experiments without thermal treatment. The addition of either methane or ethane did not substantially increase the VOC load of the effluent, but it did augment the formation of propyne in gas phase reactions around the heated substrate (potentially due to the combination of methane radicals with ethene $\mathrm{e}^{25}$ ).

VOCs are the most abundant components of the CVD effluent, but several toxic PAHs are also formed and emitted ${ }^{4}$. Eliminating thermal treatment of the feedstock gases reduced the total PAH load by an order of magnitude (Figure 4, Supporting Information). Naphthalene, fluoranthene, and pyrene were most sensitive to changes in thermal pre- 
treatment, reduced by factors of 20,60 , and 40 , respectively. The acenaphthylene, acenaphthene, fluorene, phenanthrene, and anthracene contents of the effluent were only slightly reduced by foregoing thermal pre-treatment. Interestingly, adding ethane resulted in a measurable reduction in fluoranthene and pyrene, and 1,3-butadiene-assisted CNT growth did not yield measurable fluroanthene or pyrene. In contrast, fluoranthene and pyrene were elevated in alkyne-assisted CNT syntheses relative to unassisted growths. Some have postulated that fluoranthene, whose structure resembles a CNT cap, is responsible for CNT nucleation ${ }^{19}$. The increased abundance of these four-ringed PAHs in enhanced syntheses, and their reduced presence in reactions that showed no enhancement, suggests they may have a role in CNT formation. However, further studies are needed to establish the function of PAHs in CNT formation reactions.

The total PAH content of alkyne-assisted CNT syntheses was elevated relative to unassisted growths, but was reduced by over an order of magnitude compared to traditional thermal pre-treatment techniques. Thus, the potential environmental impact of CNT manufacture can be markedly reduced without sacrificing CNT growth rate. Furthermore, selective delivery of important CNT precursor molecules will ultimately afford greater control over the reaction, as thermally generated compounds that interfere with product quality will be avoided or minimized. For example, PAHs may contribute to the formation of amorphous carbon, a significant, interfering, and difficult to remove co-product generated with almost all CNTs. We hypothesize that the reduced PAH content of alkyne-assisted syntheses will yield high purity CNTs with limited amorphous carbon coatings ${ }^{14}$, and these studies are on going. 


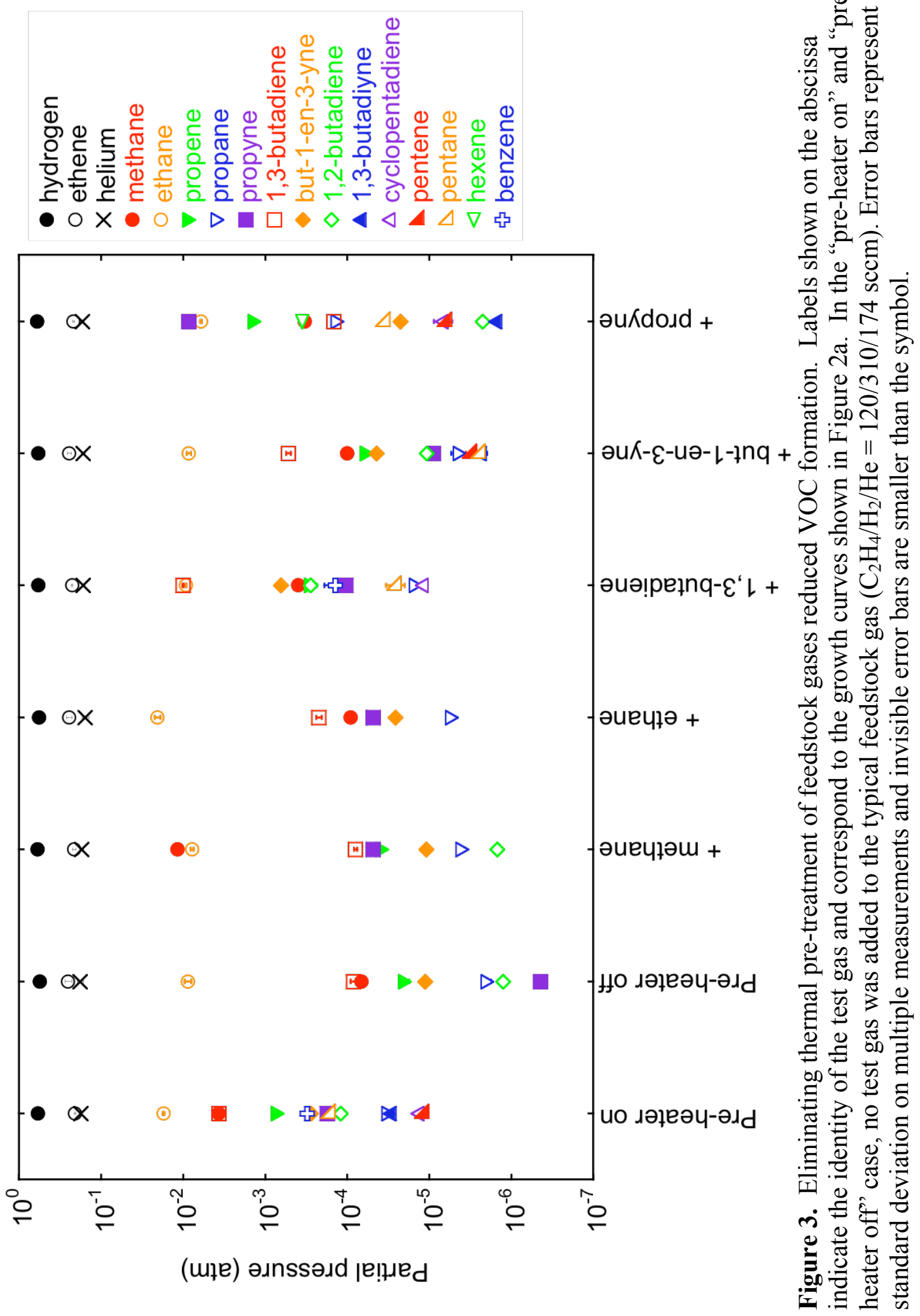




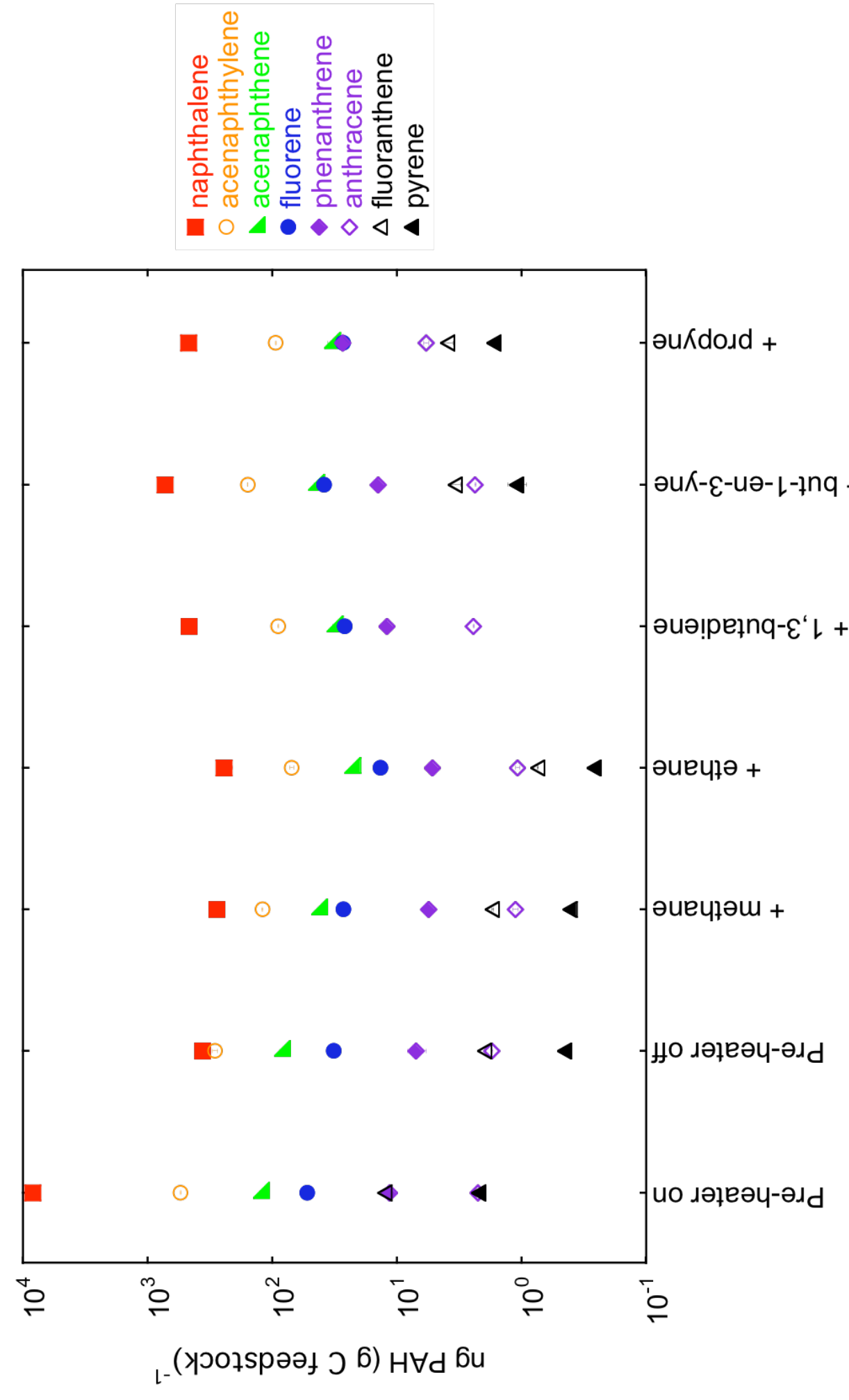

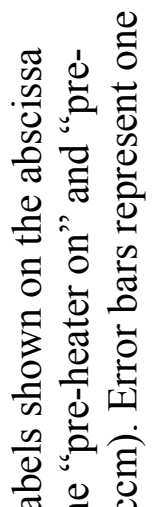

西五

몽

产 동

छ

잉

피그

$\varangle \|$

로

플 है

요

ठृ

氙记

is

के

다원

舟

엉유 종

듬.

词市

苞苛窇

छ ᄋ요

¿

ป त 휴

పี



효

$\Xi \nsubseteq$ 品

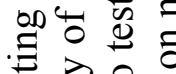

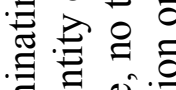

寻焉

- \&

نे

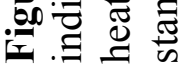




\section{Minimizing the use of ethene and hydrogen: Optimizing feedstock}

requirements. While a relatively small concentration of alkyne added to the unheated ethene feedstock accelerated CNT growth, the mass of alkyne was not sufficient to account for the mass of CNT formed, and additional compounds must have added to the growing CNT lattice. The principle feedstock, ethene, may serve as a co-reactant at the metal catalyst to form CNTs. If so, we would expect to see a reduction in the rate of CNT formation as $\mathrm{C}_{2} \mathrm{H}_{4}$ abundance is reduced. As mentioned earlier, transition-metal catalyzed cyclization reactions rely on both an alkyne and an alkene to form new carboncarbon bonds, ultimately forming unsaturated rings with carbon backbones. In these reactions, the metal must be reduced in order to have catalytic activity, and the electrons are necessary to promote bond formation. In CNT synthesis, most catalysts must be reduced (usually with $\mathrm{H}_{2}$ ) prior to $\mathrm{CNT}$ growth (except for, most notably, $\mathrm{Fe}(\mathrm{CO})_{5}$, which is used in $\mathrm{HiPCO}{ }^{\circledR}$ syntheses and is already reduced (has an $\mathrm{Fe}(0)$ oxidation state) in its native form). If the catalyst continually transfers electrons to carbonaceous reactants to form long CNTs, we would expect $\mathrm{H}_{2}$ (an electron donor) to be required for sustained catalytic activity. However, hydrogen's influence will not be strictly limited to catalytic effects, as it is also important to gas-phase reactions during CNT synthesis ${ }^{4}$. To explore the role of $\mathrm{H}_{2}$ and $\mathrm{C}_{2} \mathrm{H}_{4}$ in an alkyne-assisted CNT synthesis, we varied the concentration of each independently while fixing ethyne (the cheapest of the tested alkynes).

Furthermore, we aimed to identify the minimal $\mathrm{H}_{2}$ and $\mathrm{C}_{2} \mathrm{H}_{4}$ quantities necessary to obtain rapid, sufficient CNT formation, so as to minimize initial feedstock costs and minimize waste. 

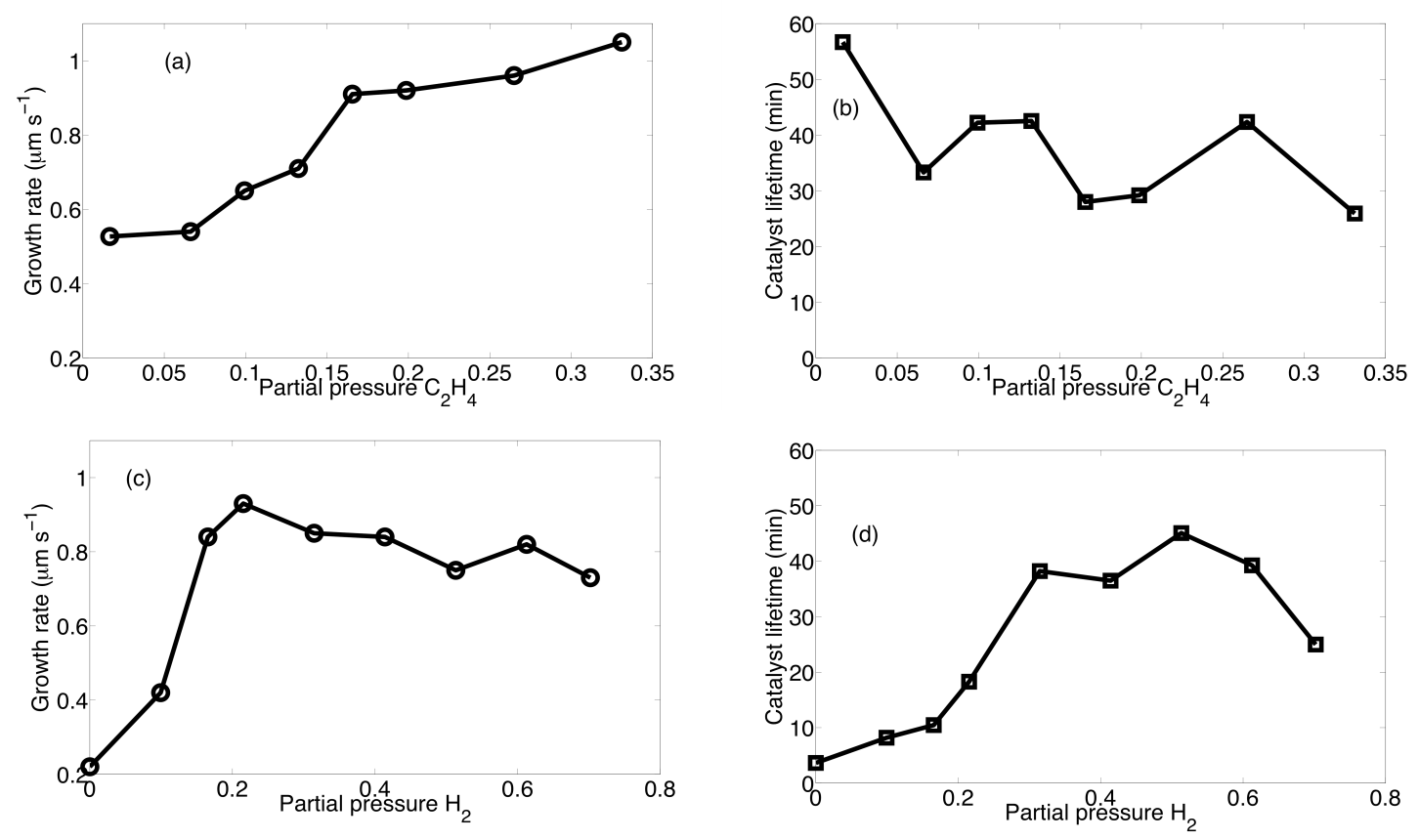

Figure 5. Effects of ethene and hydrogen on nanotube growth rate and catalyst lifetime during ethyne-assisted CNT growth. Acetone-free ethyne was delivered at $1.0 \times 10^{-3} \mathrm{~atm}$ in all experiments; when $\mathrm{C}_{2} \mathrm{H}_{4}$ was varied, $\mathrm{H}_{2}$ was constant 0.51 atm; when $\mathrm{H}_{2}$ was varied, $\mathrm{C}_{2} \mathrm{H}_{4}$ was constant at $0.20 \mathrm{~atm}$; helium was used to maintain a constant total flow rate of $604 \mathrm{sccm}$. The ethene content influenced the CNT growth rate (a), but had little effect on the catalyst lifetime (b). The hydrogen content influenced the growth rate at low partial pressure (c) and had a large impact on catalyst lifetime (d). 
As $\mathrm{C}_{2} \mathrm{H}_{4}$ abundance was reduced, a decrease in CNT growth rate was observed (Figure 5a). This is consistent with ethene having a co-catalytic role with the alkyne in the CNT formation reaction. In contrast, the abundance of $\mathrm{C}_{2} \mathrm{H}_{4}$ delivered as feedstock gas did not have a clear relationship with the catalyst lifetime (Figure 5b). Unless $\mathrm{C}_{2} \mathrm{H}_{4}$ concentration promotes significant soot formation over the catalyst, or increased $\mathrm{C}_{2} \mathrm{H}_{4}$ terminates the reaction, there is no a priori reason to expect that $\mathrm{C}_{2} \mathrm{H}_{4}$ levels would influence the duration of growth. A reduction in $\mathrm{C}_{2} \mathrm{H}_{4}$ (from 0.2 atm to $0.16 \mathrm{~atm}$ ) did not affect the growth duration (catalyst lifetime) or the growth rate appreciably. Thus, the initial feedstock concentration of ethene can be reduced by $20 \%$ without sacrificing CNT growth rate or yield.

As expected, the partial pressure of $\mathrm{H}_{2}\left(\mathrm{pH}_{2}\right)$ had a significant impact on the catalyst lifetime (Figure 5d). At low $\mathrm{pH}_{2}(<0.31 \mathrm{~atm})$, there was a sharp decrease in the catalyst lifetime, consistent with hydrogen's role as a sustained source of electrons necessary to re-reduce the catalyst after it has been oxidized (presumably by donating electrons for metal-carbon or carbon-carbon bond formation). While a minimum amount of hydrogen was necessary to sustain catalyst activity, excess $\mathrm{pH}_{2}$ reduced the catalyst lifetime. In polyethylene (polyethene) polymerization reactions, an abrupt increase in $\mathrm{pH}_{2}$ can terminate chain propagation by adding to the metal catalyst, blocking monomer addition, and it is often used to control the ultimate length of the polymer ${ }^{26-28}$. If the CNT formation reaction has analogous polymerization character, high $\mathrm{pH}_{2}$ would be expected to induce a termination event, as observed. Alternatively, termination events could be induced via protonation by water to yield a reductive coupling product (e.g., adding $\mathrm{H}$ to the $\mathrm{CNT}$ and cleaving the metal-CNT bond), and recent observations of water-induced cleavage of CNT-catalyst contact support this mechanism for termination $^{20}$.

In addition to having a substantial influence on catalyst lifetime, low $\mathrm{pH}_{2}(<0.17$ atm) affected CNT growth rate (Figure 5a). There are two potential explanations for this that rely on either gas-phase or catalyst-based reactions: (1) in the gas phase, inadequate hydrogen can limit the formation of $\mathrm{VOCs}^{4}$ (possibly denying the catalyst of a necessary 
precursor) and also promote polyethyne formation ${ }^{29}$ (which may disfavor structures with long-range order) or (2) at the catalyst, excess hydrocarbon-derived hydrogen must be removed from the growing $\mathrm{CNT}$ backbone, and $\mathrm{H}_{2}$-derived $\mathrm{H}$ radicals could abstract these, leaving a conjugated $\pi$-system in place.

Clearly, $\mathrm{H}_{2}$ serves a complicated and multi-faceted role in the CNT formation reaction. Although it is helpful to understand the dominant reaction processes of novel industrial materials, complete mechanistic knowledge is not necessary to minimize environmental damages from the process. Without compromising CNT growth rate or catalyst lifetime, the amount of input hydrogen can be reduced from 0.51 to $0.31 \mathrm{~atm}$, a $40 \%$ reduction that will translate to cost savings for the manufacturer. Earlier, we noted that a $20 \%$ reduction in ethene input was also possible (with $\mathrm{pH}_{2}=0.51$ ), and there are evident opportunities to reduce both initial feedstock costs and the total amount of carbonaceous material being vented to the atmosphere. Furthermore, eliminating the thermal treatment of feedstock gases by supplying the necessary precursors directly to the metal catalyst reduces the formation of potentially harmful and unintended by-products, saves on energetic costs associated with synthesis, and limits unnecessary damage to the environment without sacrificing production quality.

\section{ACKNOWLEDGMENTS}

A. John Hart, Eric R. Meshot, and Kevin M. Lyons are thanked for laboratory assistance and useful discussion. The authors would like to thank Jeff Seewald, Eoghan Reeves, and Sean Sylva for the use of laboratory equipment and Stephen Steiner III for helpful discussion. Sue Hess and the staff of Air Liquide America Specialty gas are thanked for their assistance and efforts to obtain rare gases. Elizabeth Forsley is thanked for laboratory assistance at the University of Michigan. We extend our gratitude to our funding sources for this project: The Woods Hole Oceanographic Institution Ocean Ventures Fund and Academic Programs Office. 


\section{REFERENCES}

[1] Eklund, P.C.; Ajayan, P.M.; Blackmon, R.; Hart, A.J.; Kong, J.; Pradhan, B.; Rao, A.; Rinzler, A.G. International Assessment of Research and Development on Carbon Nanotubes: Manufacturing and Applications, World Technology Evaluation Center Report, 2008, http://www.wtec.org/cnm.

[2] Healy, M.L.; Dahlben, L.J.; Isaacs, J.A. Environmental assessment of singlewalled carbon nanotube processes. J. Industrial Ecology 2008, 12 (3), 376-393.

[3] Thayer, A.M. Carbon nanotubes by the metric ton. Chemical \& Engineering News 2007, 85 (46), 29-35.

[4] Plata, D.L.; Hart, A.J.; Reddy, C.M.; Gschwend, P.M. Early evaluation of potential environmental impacts of carbon nanotube synthesis by catalytic chemical vapor deposition. Submitting to Environ. Sci. Technol.

[5] Joselevich, E.; Dai, H.; Liu, J.; Hata, K.; Windle, A.H. Carbon Nanotube Synthesis and Organization In Carbon Nanotubes, Advanced Topics in the Synthesis, Structure, Properties and Applications; Jorio, A., Dresselhaus, G., Dresselhaus, M.S., Eds. Springer-Verlag: New York, 2008; pp 101-164.

[6] See, C.H.; Harris, A.T. A review of carbon nanotube synthesis via fluidized-bed chemical vapor deposition. Ind. Eng. Chem. Res. 2007, 46, 997-1012.

[7] Reilly, P.T.A.; Whitten, W.B. The role of free radical condensates in the production of carbon nanotubes during the hydrocarbon CVD process. Carbon 2006, 44, 1653-1660.

[8] Raty, J-Y.; Gygi, F.; Galli, G. Growth of carbon nanotubes on metal nanoparticles: A microscopic mechanism from ab initio molecular dynamics simulations. Phys. Rev. Lett. 2005, 95 (9), 096103(4).

[9] Kanzow, H.; Ding, A. Formation mechanism of single-wall carbon nanotubes on liquid-metal particles. Phys. Rev. Lett. 1999, 60 (15), 11180-11186.

[10] Han, J-H.; Graff, R.A.; Welch, B.; Marsh, C.P.; Franks, R.; Strano, M.S. A mechanochemical model of growth termination in vertical carbon nanotube forests. ACS Nano 2008, 2 (1), 53-60.

[11] Hofmann, S.; Sharma, R.; Ducati, C.; Du, G.; Mattevi, C.; Cepek, C.; Cantoro, M.; Pisana, S.; Parvez, A.; Cervantes-Sodi, F.; Ferrari, A.C.; Dunin- 
Borkowski, R.; Lizzit, S.; Petaccia, L.; Goldoni, A.; Robertson, J. In situ observations of catalyst dynamics during surface-bound carbon nanotube nucleation. Nano Lett. 2007, 7 (3), 602-608.

[12] Xiang, R.; Yang, Z.; Zhang, Q.; Luo, G.; Qian, W.; Wei, F.; Kadowaki, M.; Einarsson, E.; Maruyama, S. Growth deceleration of vertically aligned carbon nanotube arrays: Catalyst deactivation or feedstock diffusion controlled? $J$. Phys. Chem. C 2008, 112, 4892-4896.

[13] Hart, A.J.; van Laake, L.C.; Slocum, A.H. Desktop growth of carbon nanotube monoliths with in situ optical imaging. Small 2007, 3 (5), 772-777.

[14] Meshot, E.R.; Ng, J.; Hart, A.J. Co-optimizing carbon nanotube synthesis: Controlling diameter, quality, and growth rate of vertically-aligned carbon nanotubes by decoupled thermal treatment of precursor and catalyst. Submitted to ACS Nano.

[15] Gutowski, T.G.; Branham, M. Department of Mechanical Engineering, Massachusetts Institute of Technology, Personal Communication, 2008.

[16] Gutowski, T.G.; Branham, M.S.; Dahmus, J.B.; Jones, A.J.; Thiriez, J.; Thiriez, A.; Sekulic, D.P. Thermodynamic analysis of resources used in manufacturing processes. Environ. Sci. Technol. 2009, doi: 10.1021/es8016655.

[17] van Laake, L.C.; Hart, A.J.; Slocum, A.H. A suspended heated silicon platform for rapid thermal control of surface reactions with application to carbon nanotube synthesis. Review of Scientific Instruments 2007, 78, 083901.

[18] Vasenkov, A.V.; Sengupta, D.; Frenklach, M. Multiscale modeling catalytic decomposition of hydrocarbons during carbon nanotube formation. J. Phys. Chem. B 2009, 113 (7), 1877-1882.

[19] Eres, G.; Kinkhabwala, A.A.; Cui, H.; Geohegan, D.B.; Puretzky, A.A.; Lowndes, D.H. Molecular beam-controlled nucleation and growth of vertically aligned single-wall carbon nanotube arrays. J. Phys. Chem. B. 2005, 109 (35), 1668416694.

[20] Yamada, T.; Maigne, A.; Yudasaka, M.; Mizuno, K.; Futaba, D.N.; Yumura, M.; Iijima, S.; Hata, K. Revealing the secret of water-assisted carbon nanotube synthesis by microscopic observation of the interaction of water on the catalysts. Nano Letters. 2008, 8 (12), 4288-4292. 
[21] Jeganmohan, M.; Cheng, C-H. Cobalt- and nickel-catalyzed regio- and stereoselective reductive coupling of alkynes, allenes, and alkenes with alkenes. Chem. Eur. J. 2008, 14, 10876-10886.

[22] Sugihara, T.; Yamaguchi, M.; Nishizawa, M. Advances in the Pauson-Khand reaction: Development of reactive cobalt complexes. Chem. Eur. J. 2001, 7(8), 1589-1595.

[23] Shi, Y.; Peterson, S.M.; Haberaecker III, W.W.; Blum, S.A. Alkynes as Stille reaction pseudohalides: Gold- and Palladium-cocatalyzed synthesis of tri-and tetra-substituted olefins. JACS Comm. 2008, 130, 2168-2169.

[24] Joosten, A.; Soueidan, M.; Denhez, C.; Harakat, D.; Hélion, F.; Namy, J-L.; Vasse, J-L.; Szymoniak, J. Multimetallic zirconocene-based catalysis: Alkyne dimerization and cyclotrimerization reactions. Organometallics, 2008, 27, $4152-4157$.

[25] Stein, S.E.; Walker, J.A.; Suryan, M.M.; Fahr, A. A new pathway to benzene in flames. Twenty-third Symposium (International) on Combustion. The Combustion Institute, Pittsburh, 1990, pp. 85-90.

[26] Choi, K.Y.; Ray, W.H. Polymerization of olefins through heterogeneous catalysis. II. Kinetics of gas phase propylene polymerization with Ziegler-Natta catalysts. J. Appl. Polymer Sci. 1985, 30, 1065-1081.

[27] Jaber, I.A.; Ray, W.H. Polymerization of olefins through heterogeneous catalysis. XIV. The influence of temperature in the solution copolymerization of ethylene. J. Appl. Polymer Sci. 1993, 50, 201-215.

[28] Jaber, I.A.; Ray, W.H. Polymerization of olefins through heterogeneous catalysis. XIV. The influence of pressure in the solution copolymerization of ethylene. $J$. Appl. Polymer Sci. 1993, 50, 217-231.

[29] Stein, S.E.; Fahr, A. High-temperature stabilities of hydrocarbons. J. Phys. Chem, 1985, 89 (17), 3714-3725. 


\section{SUPPORTING INFORMATION}

\section{Linear and log-linear relationships between CNT precursor molecule abundance and CNT growth rate}

The hyperbolic relationship (Figure 1) between propyne abundance and growth rate and can be used to determine the asymptotic maximum rate $\left(v_{\max }\right)$ and a characteristic concentration of propyne (concentration at half of the maximum growth rate, $C_{\text {half-max }}$ ). The maximum possible rate, $13.5 \mathrm{um} \mathrm{s}^{-1}$, was greater than the observed maximum VAMWCNT growth rate of $9.1 \mathrm{um} \mathrm{s}^{-1}$, suggesting that higher VA-MWCNT formation rates were possible. (The characteristic propyne concentration at half of the maximum growth rate was $7.5 \times 10^{-4} \mathrm{~atm}$ ). The saturating behavior, where product (i.e., VA-MWCNT) formation does not continually increase with higher substrate (i.e., propyne) concentration, is characteristic of surface-catalyzed reactions ${ }^{l}$.

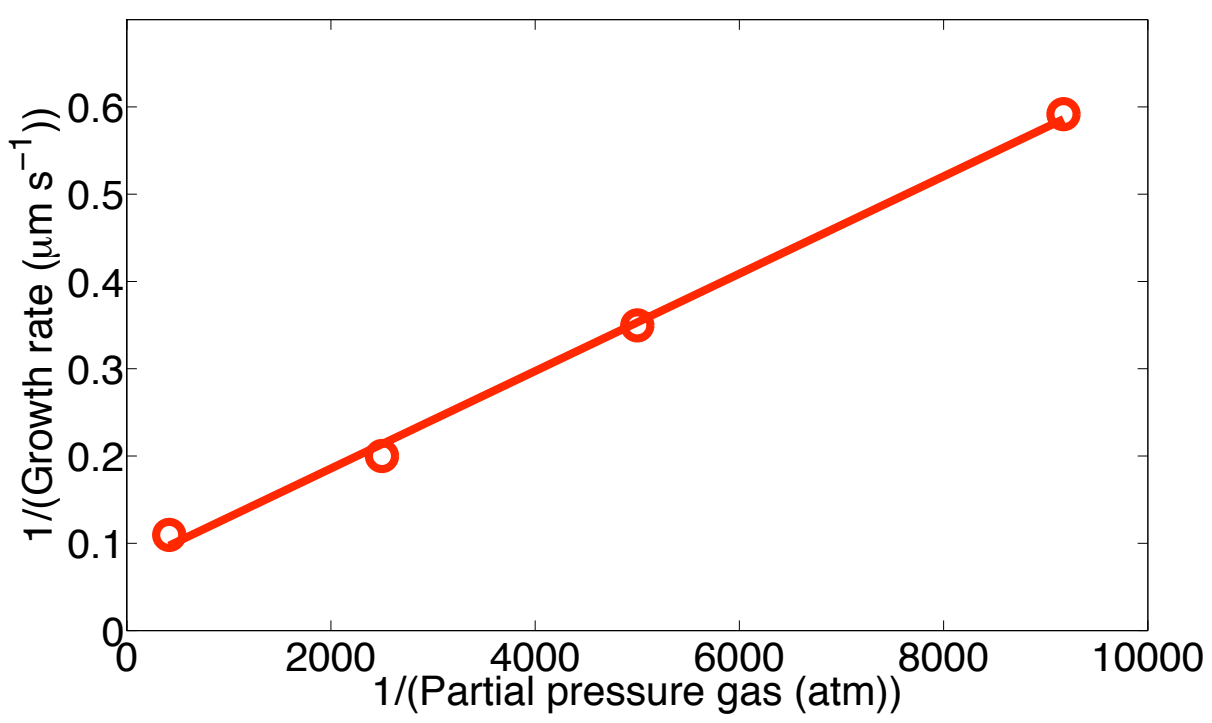

Figure S1. Kinetic treatment of surface-catalyzed reactions. The relationship between VA-MWCNT formation and propyne abundance (inverse of propyne abundance plotted on the $\mathrm{x}$-axis and the inverse of the growth rate plotted on the $\mathrm{y}$-axis) was used to extract relevant kinetic parameters. Here, the y-intercept is equal to the inverse of the maximum possible growth rate $\left(v_{\max }\right)$. The slope is equal to the concentration of propyne at half of the maximum growth rate $\left(C_{\text {half-max }}\right)$ divided by the maximum growth rate $\left(v_{\max }\right)$. 


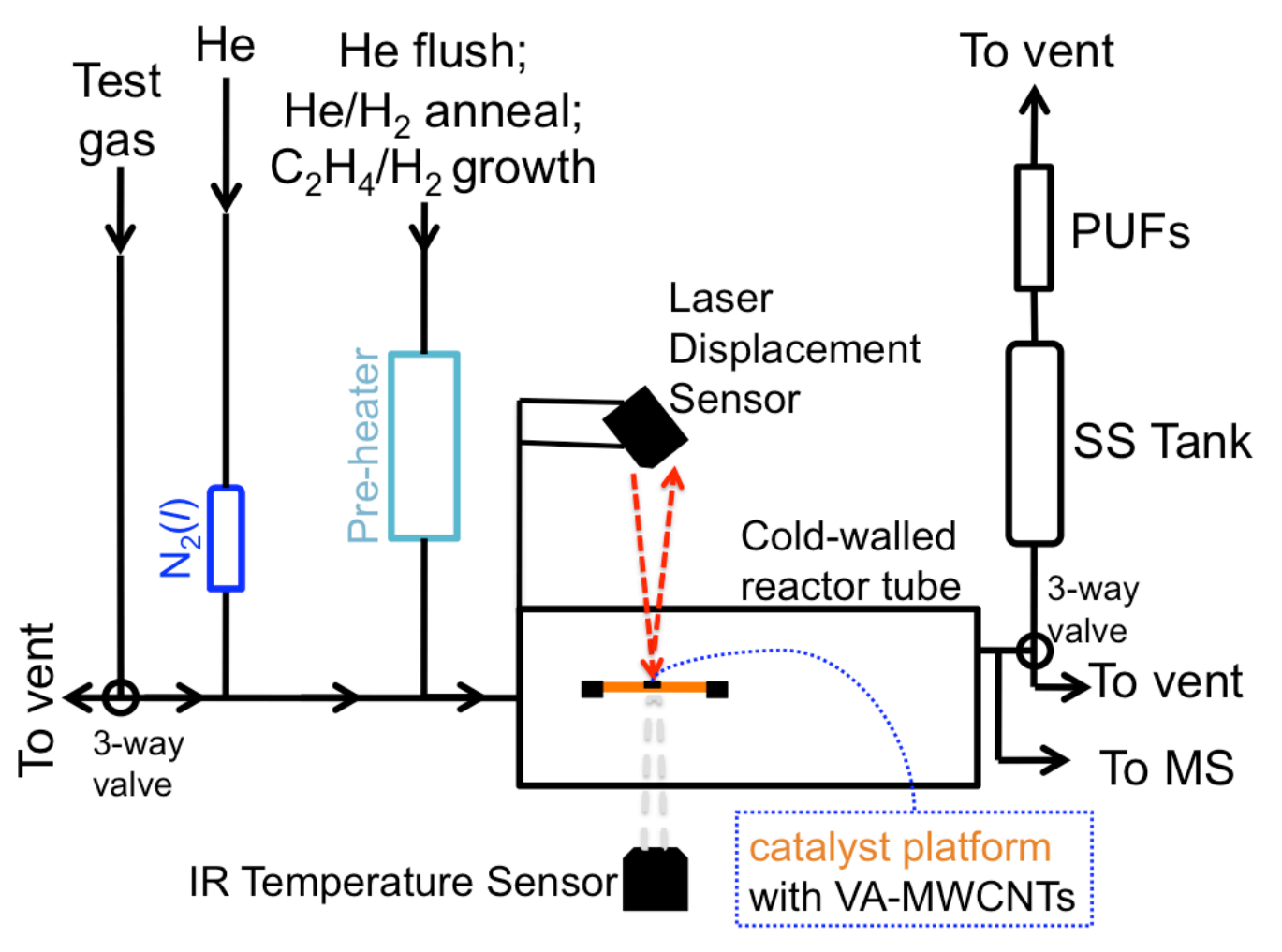

Figure S2. Schematic diagram of experimental apparatus. Traditional growth feedstock gases $\left(\mathrm{C}_{2} \mathrm{H}_{4} / \mathrm{H}_{2}\right)$ and annealing gases $\left(\mathrm{He} / \mathrm{H}_{2}\right)$ are introduced via a resistively heated preheater tube that was operated in two modes: (1) "on" at $1000^{\circ} \mathrm{C}$ and (2) "off" at room temperature $\left(21^{\circ} \mathrm{C}\right)$. With the pre-heater off, test gases (e.g., 1,3-butadiene, ethyne, propyne, 1-butyne, but-1-en-3-yne, methane, ethane, or benzene) were introduced during the growth phase via a secondary input line, which could be flushed to a vent via a 3-way valve during the flush and anneal phases. The flow rate of the test gas was balanced with an additional helium line that was cryogenically purified with a Porasil-C column immersed in liquid nitrogen. For all experiments, the total flow of gas $\left(\mathrm{C}_{2} \mathrm{H}_{4}+\mathrm{H}_{2}+\mathrm{He}+\right.$ test gas $)$ was $604 \mathrm{sccm}$, except where noted. These were introduced to a cold-walled quartz reactor tube that housed a resistively heated silicon platform, which supported the vertically aligned multi-wall carbon nanotube (VA-MWCNT) catalyst substrate. The temperature of the platform was monitored and controlled (via feedback) by an infrared (IR) temperature sensor, and the growth rate of the VA-MWCNT forest was monitored using a laser displacement sensor. Effluent gases were continually monitored by online mass spectrometry (MS, $2 \mathrm{sccm}$ sampling rate), and subsequently flushed to either a vent (during anneal and flush) or though a stainless steel sampling tank (SS Tank, to collect VOCs, $\mathrm{He}$, and $\mathrm{H}_{2}$ ), quartz fiber filters (to collect particles $>0.2 \mathrm{um}$ ), and polyurethane foams (PUFs, to collect PAHs). 


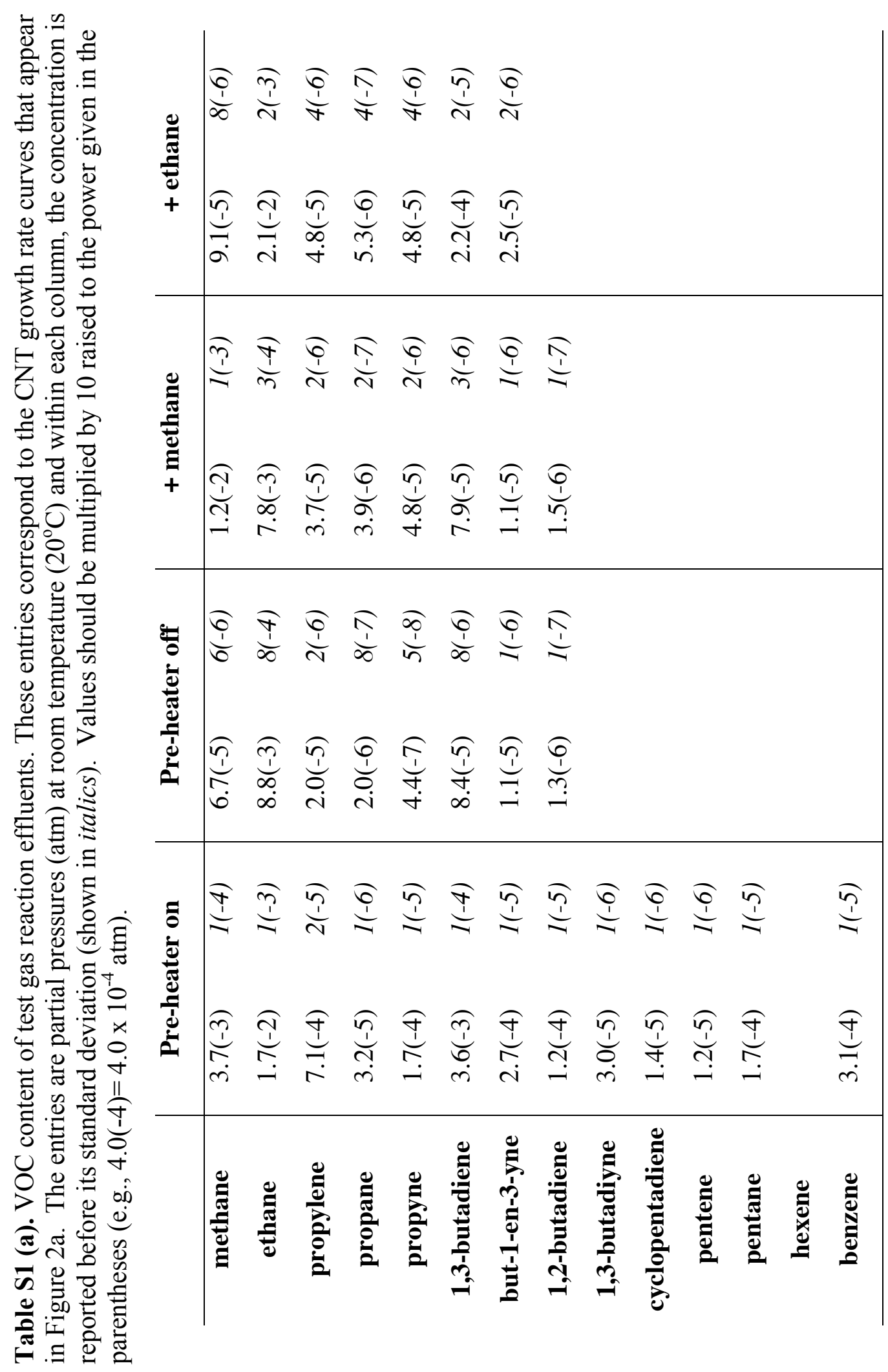




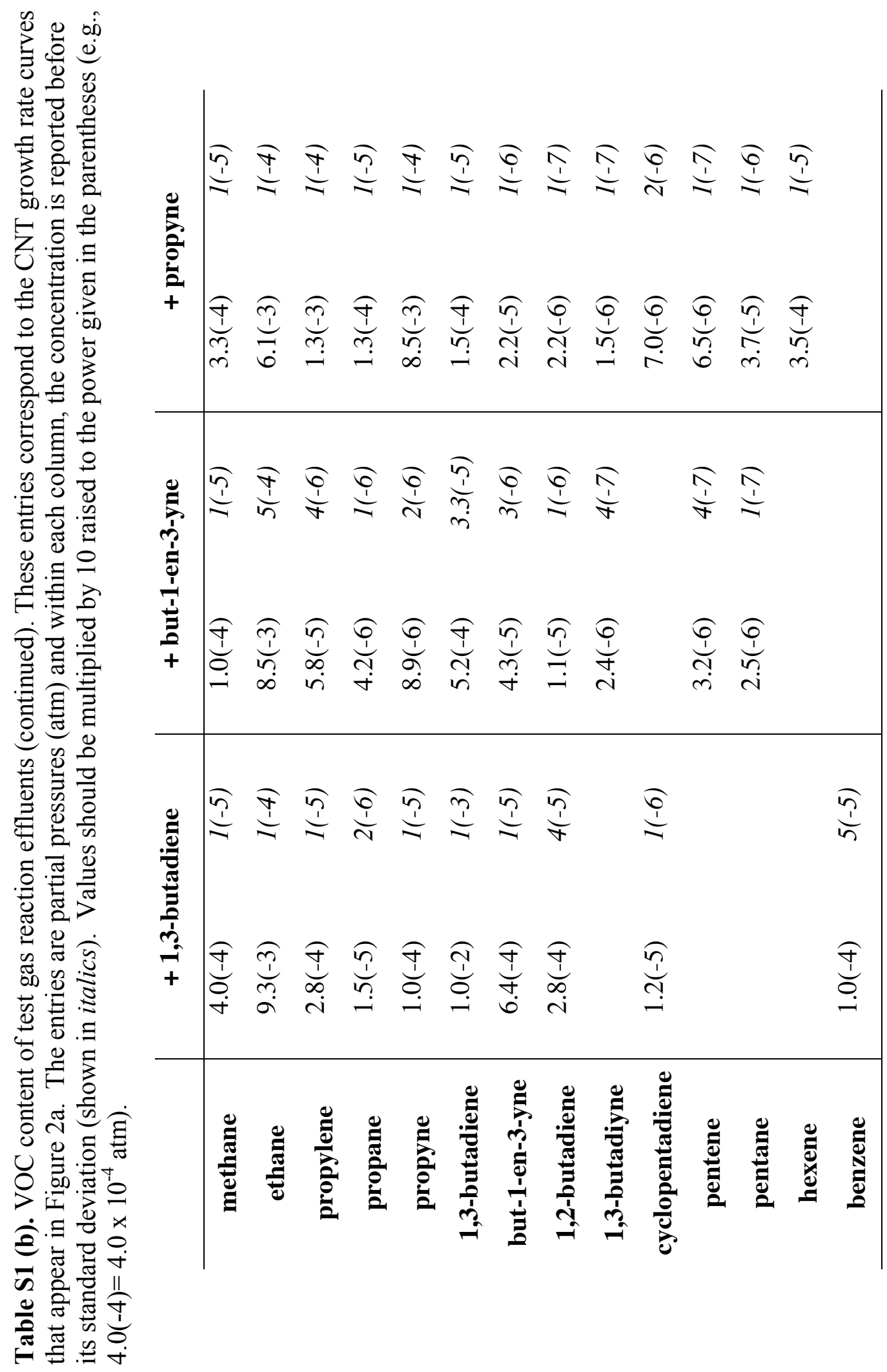




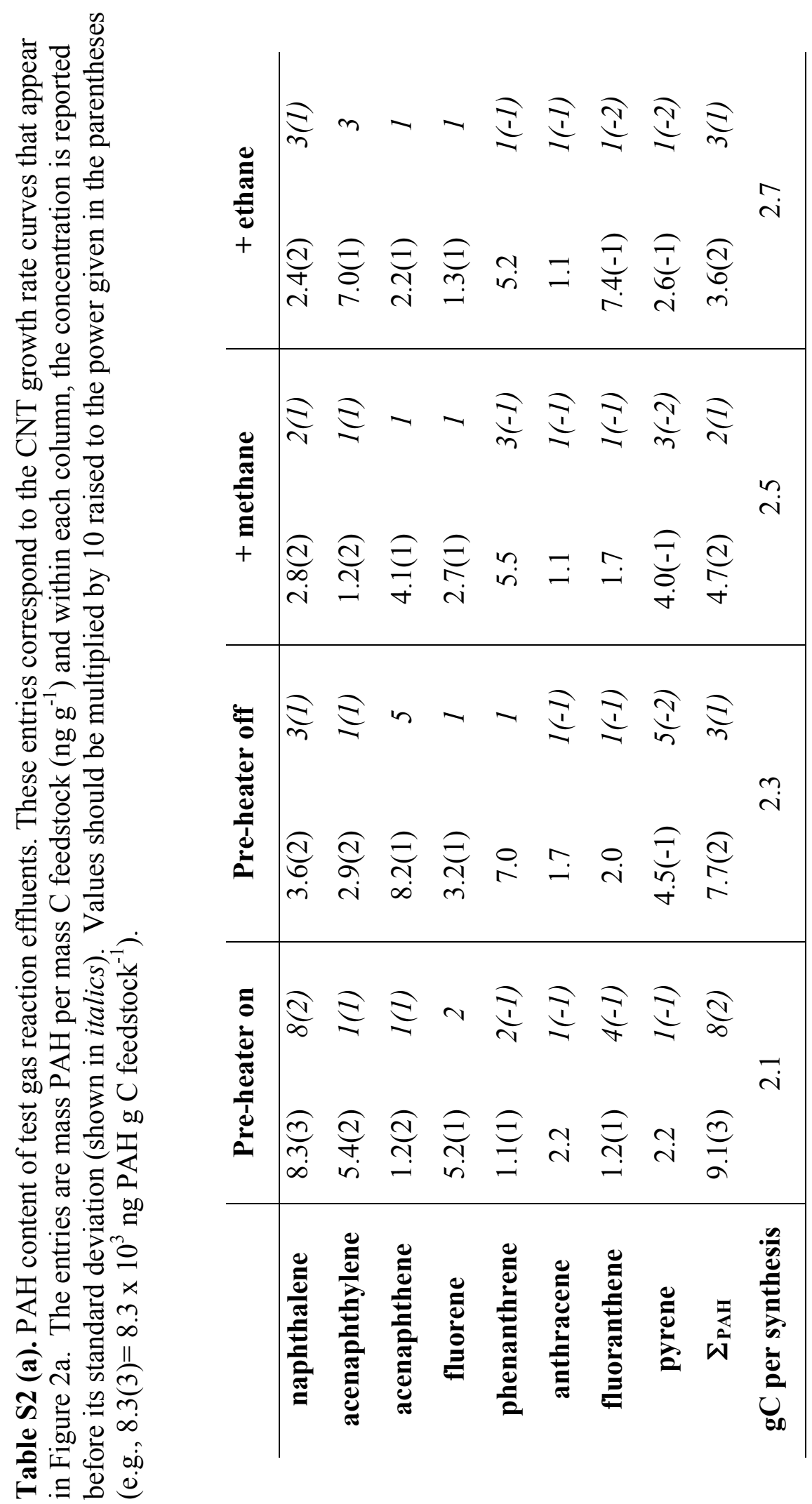




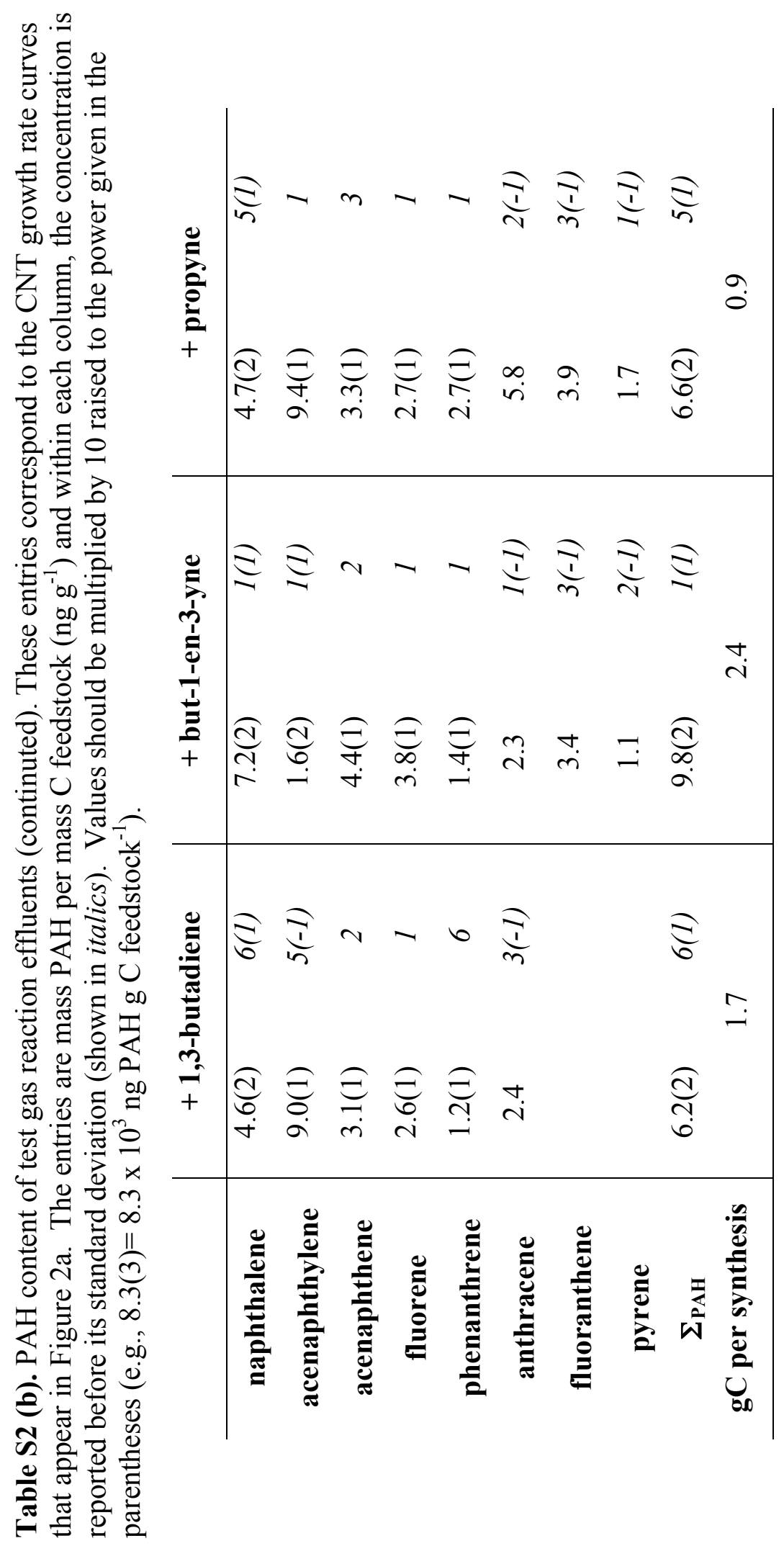




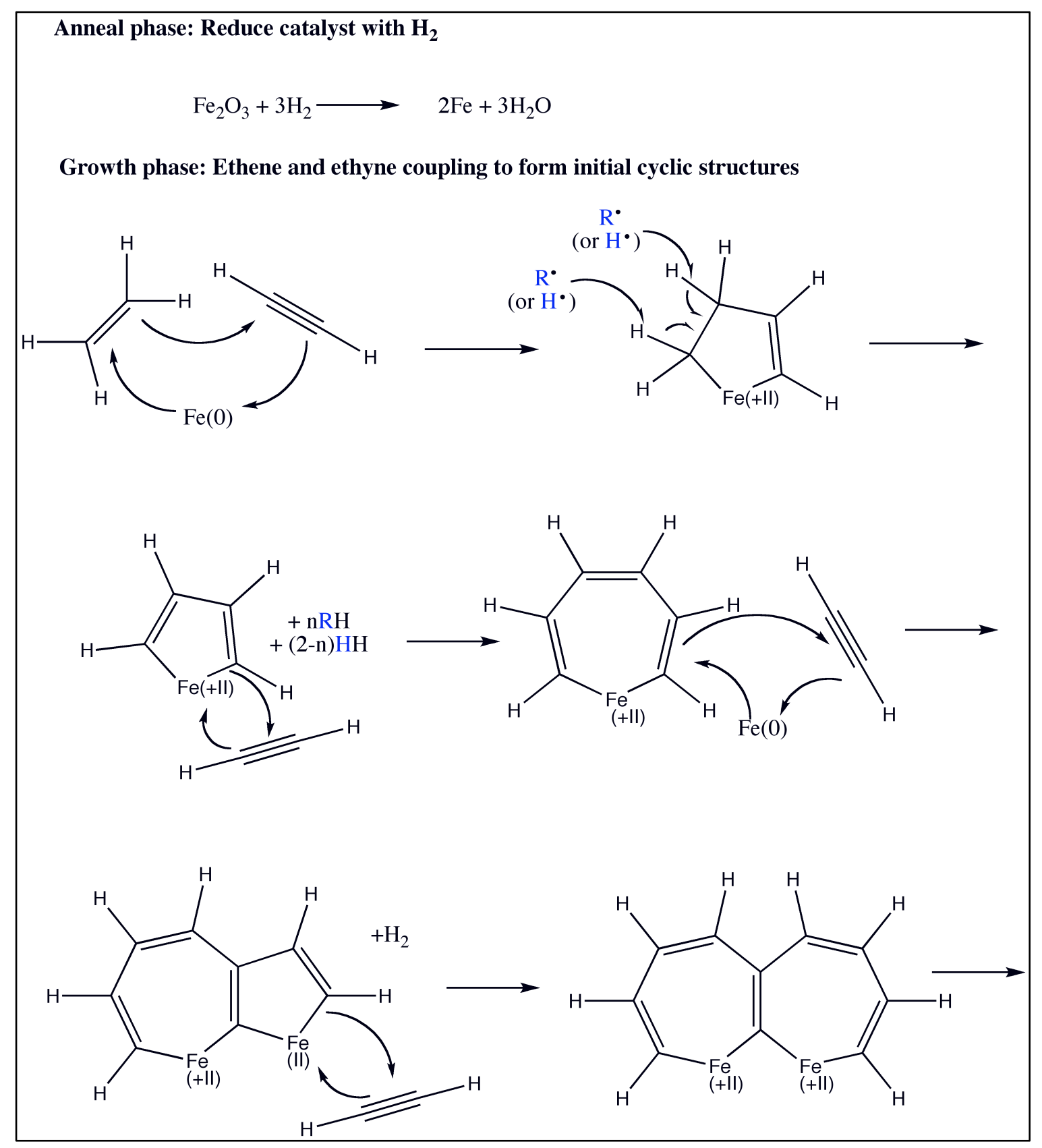

Figure S3(a). Proposed CNT growth mechanism. During the anneal phase of CNT production, the catalyst was reduced and subject to morphological changes that yield metal nanoparticles ${ }^{2}$. There is precedence in the literature for both (1) coupling reactions between alkynes and alkenes that proceed via metallocycles (e.g., Jeganmohan and $\mathrm{Chen}^{3}$ ) and (2) alkane insertion to a growing C-chain. Excess hydrogen atoms must be liberated without increasing the $\mathrm{C}-\mathrm{C}$ bond order, but no mechanism for this is proposed here. 
Growth phase continued: Elongation by sequential alkyne or alkene addition (alkyne shown)
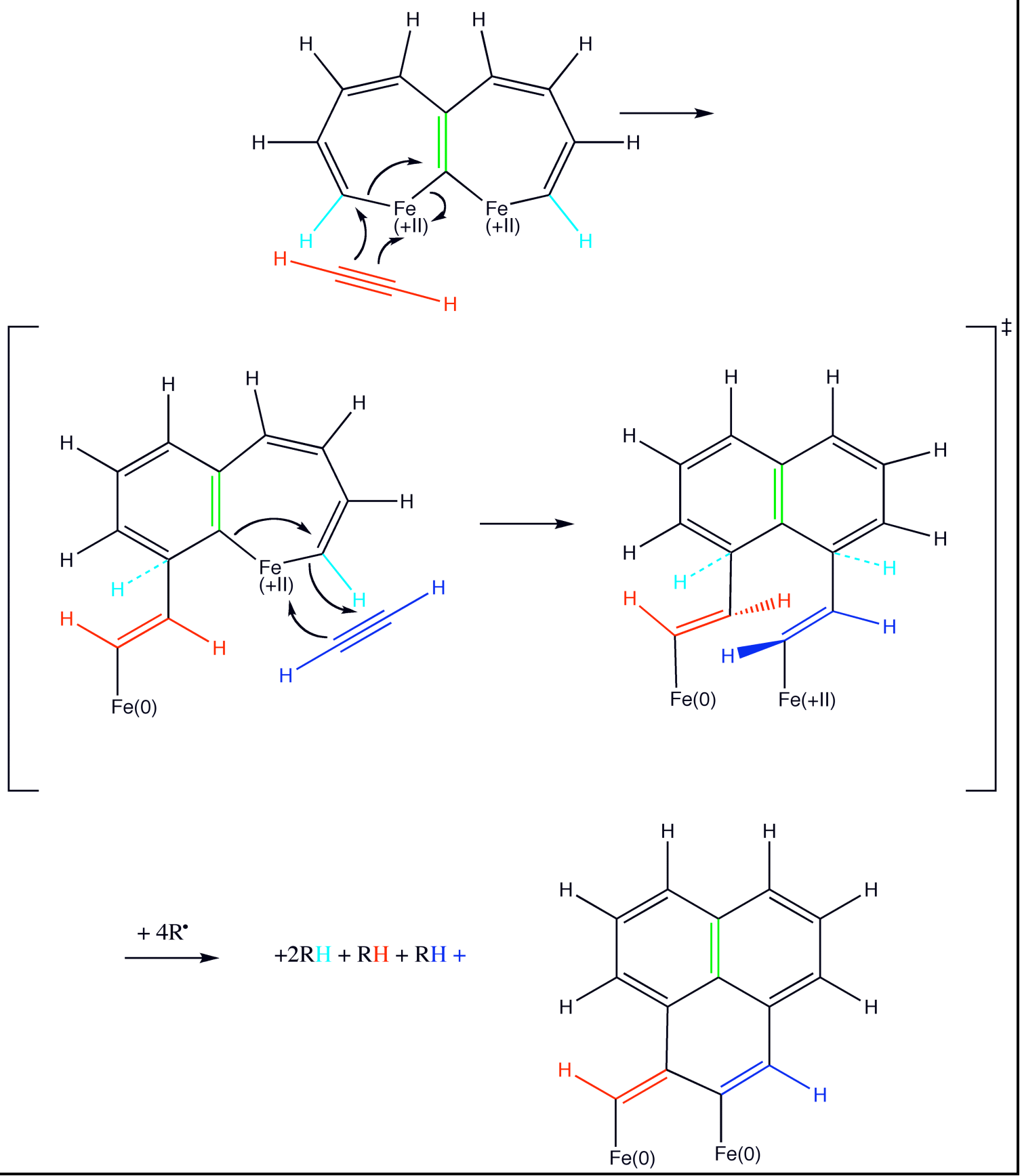

Figure S3(b). Proposed CNT growth mechanism (continued). Here, we present possible propagation steps, but these need to be vetted experimentally. An unstable transition state is depicted here, where excess hydrogen atoms are liberated by reaction with radicals $(\mathrm{R} \bullet)$ and resultant electrons add to the CNT lattice and ultimately reduce the metal catalyst. Colors are used to guide the eyes. 


\section{Termination events: Net $\mathrm{H}_{2}$ addition}

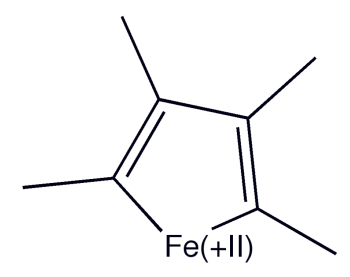

Reductive elimination

by excess $\mathrm{H}_{2}$

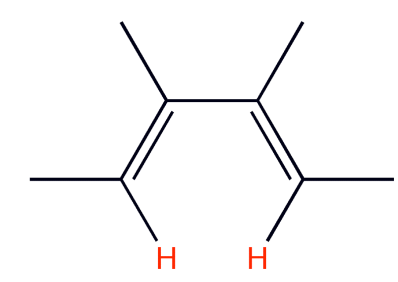

$+\mathrm{Fe}(+\mathrm{II})$

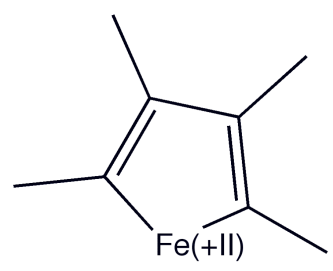

Reductive elimination

by excess $\mathrm{H}_{2} \mathrm{O}$

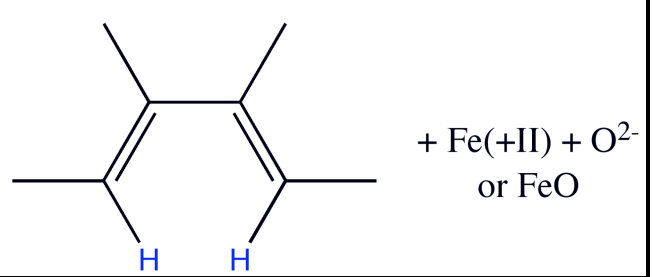

Figure S3(c). Proposed CNT termination mechanism. There is precedence in the literature for water to cleave catalyst-CNT bonds ${ }^{4}$, and our results suggest that excess gas-phase hydrogen could terminate growth. Colors are used to guide the eyes.

\section{REFERENCES}

[1] Atkins, P. Physical Chemistry, $6^{\text {th }}$ Ed., Chapter 8: Processes at Solid Surfaces. W.H. Freeman and Company. 1997. New York. pp. 866-868.

[2] Hart, A.J. Chemical, mechanical, and thermal control of substrate-bound carbon nanotube growth. MIT Ph.D. Thesis. 2006

[3] Jeganmohan, M.; Cheng, C-H. Cobalt- and nickel-catalyzed regio- and stereoselective reductive coupling of alkynes, allenes, and alkenes with alkenes. Chem. Eur. J. 2008, 14, 10876-10886.

[4] Yamada, T.; Maigne, A.; Yudasaka, M.; Mizuno, K.; Futaba, D.N.; Yumura, M.; Iijima, S.; Hata, K. Revealing the secret of water-assisted carbon nanotube synthesis by microscopic observation of the interaction of water on the catalysts. Nano Letters. 2008, 8 (12), 4288-4292. 


\section{CHAPTER 6:}

REFLECTIONS ON AND FORECASTS OF THIS WORK:

CONTRIBUTIONS, CRITICISMS, PRECEDENT, AND OPPORTUNITIES

By

Desirée L. Plata 


\section{CONTRIBUTIONS}

In our history, no industrially important chemical has been produced, used, and disposed of without some release to the environment. Furthermore, chemical manufacturing procedures are often designed to maximize material performance and minimize production costs, with little attention devoted to environmental impact mitigation. This model results in postponed recognition of malignant environmental and public health effects, and at an advanced stage of industrial development, it is very difficult to arrest, let alone rectify, the resultant damages. Aware of this pattern and poised at the expansion of the carbon nanotube (CNT) industry, engineers and environmental scientists have a unique opportunity to alter the future approach to the development of all materials: by incorporating environmental objectives prior to industrial scale-up of synthesis. In doing so, we will not only prevent unnecessary harm to the earth and human health, but will also preserve the public's confidence in a field that promises many benefits to society.

To achieve this vital goal, I investigated the potential impacts of promising novel materials, CNTs, on the Earth system. There are two primary objectives to this research: (1) to assess emissions from a common CNT production method and use improved mechanistic understanding to reduce the release of compounds of environmental concern (e.g., green house gases, toxins, and materials that form secondary pollutants, such as smog and aerosols), and (2) to develop a method to monitor the fate of and biological exposure to CNTs in complex environmental matrices (e.g., air, water, soil, and sediment).

Potential impacts from the manufacture of CNTs. The industrially favored production method, carbon vapor deposition (CVD), is plagued by inefficiency. Less than $3 \%$ of the source carbon feedstock becomes CNT product, while the remaining $\sim 97 \%$ is often vented to the atmosphere ${ }^{a}$. This material was almost completely

\footnotetext{
${ }^{a}$ Some CNT manufacturers reuse their effluents a few times each day, ultimately venting unused materials. Alternatively, some manufacturers "flame" their effluents, in an attempt to convert unused material to $\mathrm{CO}_{2}$.
} 
uncharacterized prior to my dissertation research, but I hypothesized that the reaction conditions (heating carbonaceous gas to high temperatures, $500-1200{ }^{\circ} \mathrm{C}$ ) would result in the formation of volatile organic compounds (VOCs), polycyclic aromatic hydrocarbons (PAHs), and soot. All of these raise environmental and public heath concern: photoactive VOCs contribute to smog and lower atmosphere ozone formation, which can exacerbate respiratory disease; select PAHs are toxic; and soot influences respiratory health as well as the radiative heat balance of the atmosphere. Using a representative, laboratory-scale CVD reactor (fed with ethene and hydrogen), I developed a collection apparatus that enabled detection of compounds (PAHs) at parts per trillion levels; six orders of magnitude lower than previously possible for these systems. My results showed, as expected, that VOCs, PAHs, soot, and the potent greenhouse gas, methane, were formed and released.

Assuming that the effluent-to-CNT ratio would be the same for an industrial-scale synthetic process ${ }^{\mathrm{b}}$, I estimated contributions to the national VOC and PAH emissions due to two future CNT-based applications. I found that, compared to national emissions, the quantity of VOCs and PAHs produced from CNT production (for thermal interface materials (TIMs) and flat panel displays (FPDs) only) would be relatively small. However, these emissions could become important on a local scale. In particular, assuming that $20 \%$ of of TIM and FPD production occurred in Houston, TX (one of the United States' most industrialized cities), emissions of the toxic hazardous air pollutants 1,3-butadiene and benzene would increase by almost $50 \%$ and over $3 \%$, respectively.

I found these potential outputs to be unacceptable (for reasons discussed later in "Criticism" section), and I sought to refine the CNT formation process to reduce the initial production of environmentally concerning compounds. I noted that increasing the temperature of thermal treatment of the reactant gasses (ethene and hydrogen) increased

\footnotetext{
${ }^{\mathrm{b}}$ I recognize that this assumption likely flawed, but it is the best estimate that can be developed until industrial scale processes are fully developed. Further, the estimate is intended to guide the selection of synthetic practices with minimal environmental impact by providing future emissions projections, rather than assert that manufacturing practices will be unchanged or emissions-to-CNT ratios will be fixed.
} 
the quantity of VOCs and PAHs, but at the same time, increased CNT formation rate. I hypothesized that only select thermally generated compounds were necessary for rapid CNT growth, while others were interfering chemicals that might reduce CNT quality and increase negative environmental impacts of the production. By avoiding thermal treatment of the feedstock gas and, instead, delivering the critical precursors directly to the CNT catalyst, I expected that rapid, high-quality, low-polluting, lower energy consumption CNT growth was possible.

By monitoring the in situ kinetics of CNT formation (using a laser displacement measurement of the height of vertically aligned CNTs) and corresponding gas analysis, I was able to identify which of the thermally generated products were important for the CNT growth. Small alkyne precursors were most effective at accelerating CNT growth, and the data suggest that they are acting in concert with ethene to form CNTs. Additionally, I found that hydrogen plays a role in both sustaining catalyst activity and gas phase reactions. Nevertheless, the input levels of ethene and hydrogen could be reduced by 20 and $40 \%$, respectively, without sacrificing CNT growth. This represents an automatic savings in both costs and emissions. Furthermore, the VOC and PAH emissions due to alkyne-assisted, unheated CNT formation were reduced by more than an order of magnitude. The energetic costs of the synthesis will drastically decrease, which will translate into $\mathrm{CO}_{2}$ savings if the electricity source is carbon-derived. In addition, I expect that the product quality of CNTs will increase (i.e., reduced coating of amorphous carbon, which can interfere with CNT electrical conductivity) when PAH formation decreases, and early results suggest this may be the case (Figure 1). If this is verified, then not only were emissions, energetic costs, and feedstock costs reduced, but the product quality may also be improved. Thus, it would seem only sensible for a manufacturer to adopt this environmentally conscientious synthetic approach. 


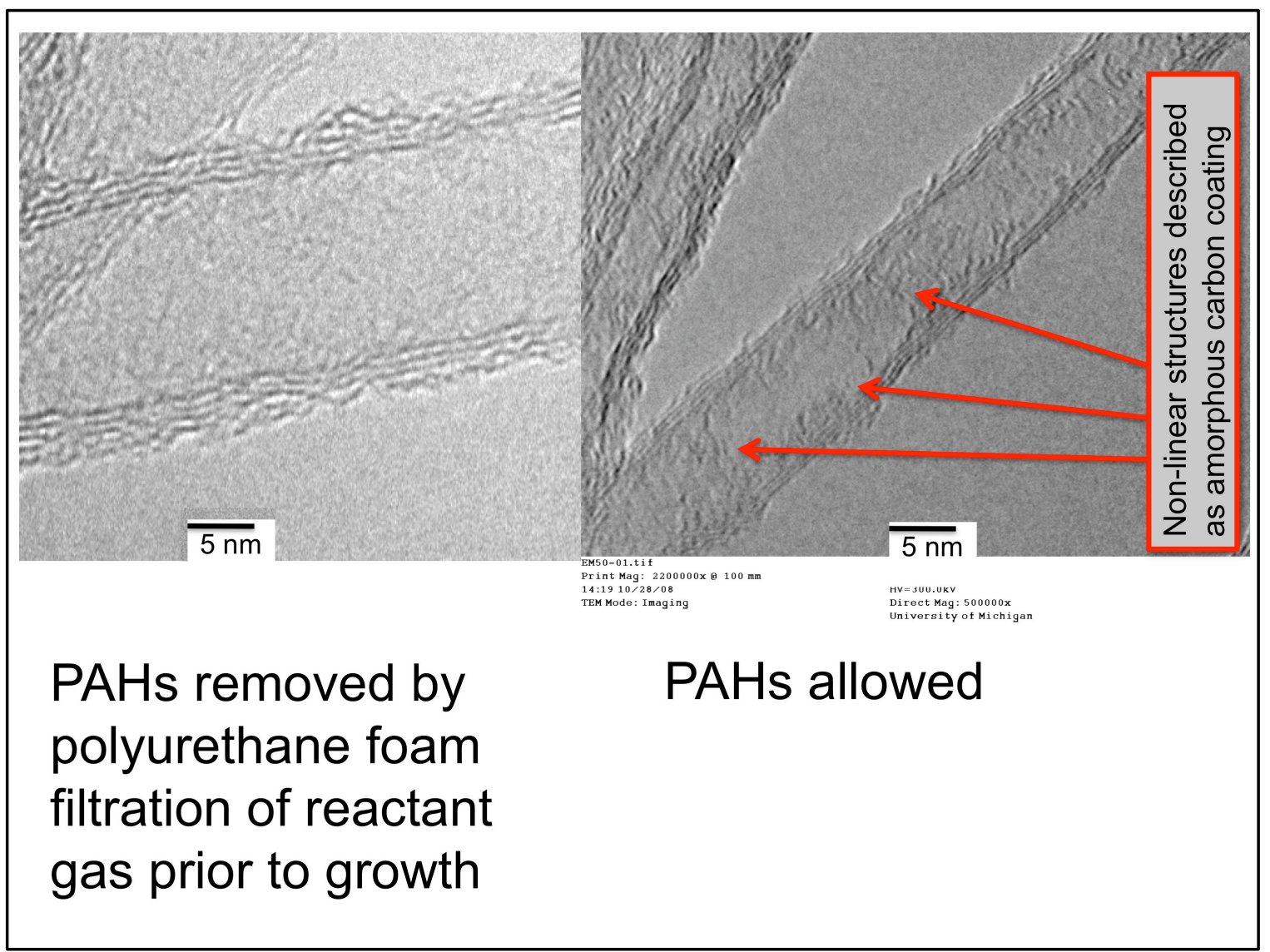

Figure 1. High-resolution transmission electron micrograph of MWCNTs grown in the absence (left panel) and presence (right panel) or PAHs. I hypothesize that PAHs may contribute to the formation of amorphous carbon on CNTs. As an initial, first-order test of this theory, I filtered PAHs from the post-heated ethene/hydrogen feedstock prior to delivery to the CNT metal catalyst. In the left panel (no PAHs), there was no noticeable deposition of amorphous carbon. In the right panel, nanotubes grown in the presence of PAHs had amorphous carbon deposits (non-linear structures on top of the CNT). Note that the right panel tubes were grown under slightly different synthetic conditions (hence the smaller diameter), but are representative of typically observed amorphous coatings. Studies to vet this hypothesis are ongoing. (Images courtesy of Matteo Seita (ETH Zurich) and Eric Meshot (University of Michigan)). 
Developing a method to detect CNTs in the natural world. There is some concern that CNTs themselves, which have demonstrated toxicity ${ }^{l-6}$, are released in the effluent of CVD reactors. However, there was no established method for detecting CNTs in complex environmental matrices (e.g., air, water, sediments) at the onset of this investigation. This limitation precludes the study of CNT fate in the environment, limits the ability of government agencies to enforce regulation, and prevents the assessment of biological exposure to CNTs. A large portion of my dissertation research was dedicated to overcoming this challenge.

I developed a method for CNT detection using thermal gravimetric analysis-mass spectrometry (TGA-MS). First, I showed that commercially available SWCNTs exhibit a wide range in oxidation temperatures, perhaps related to the type of metal catalyst present with the CNT. All SWCNTs were less thermally stable than black carbon (BC) soot, more thermally stable than methylcellulose, and exhibited significant to marginal codegradation with environmental matrices (e.g., aerosol concentrates and sediments, respectively). Using a SWCNT sample that exhibited minimal co-degradation with natural organic phases, I monitored gases evolved during a thermal degradation in either dry air or hydrogen atmospheres. As SWCNTs have very low hydrogen and oxygen contents relative to other natural materials, SWCNTs exhibited very low $\mathrm{H}_{2} \mathrm{O} / \mathrm{CO}_{2}$ ratios in air and low $\mathrm{H}_{2} \mathrm{O} / \mathrm{C}_{2} \mathrm{H}_{4}$ ratios in hydrogen. Using these depletions, I was able to detect quantities as low as $10 \mathrm{ug}$ SWCNT in sedimentary samples (40 mg sample size), which was set by co-oxidation of organic phases (in air experiments) and leaks of air (which has an interfering mass fragment) into the TGA-MS (in hydrogen experiments). While this method is not yet sensitive enough for current environmental CNT levels ( $\mathrm{pg} \mathrm{m}^{-3}$ of air, $\mathrm{pg} \mathrm{g}^{-1}$ of sediment, based on results of a dynamic mass balance model that I developed in Chapter 1 of this thesis), it is sufficient for detection of CNTs in production facility air and perhaps near effluent towers. TGA-MS is a high-throughput, low-cost, accessible method that could be used by many individuals in industry, government, and academia to put an upper bound on the potential releases of CNTs. If the detection limit was 
exceeded, measures could be made to reduce the release of CNTs from these potential point sources.

In spite of the current detection limit, there are uses for the methods presented here. For example, all of the current CNTs contained in consumer products sold today are sold as CNT-epoxy or CNT-polymer composites. CNTs are fairly concentrated in these samples, and so, the CNT content of a product might be tested using the present TGA-MS method. In addition, all studies of carbon nanomaterial release from production facilities so far have relied on light-scattering techniques to detect nanoparticles. These methods are unable to distinguish between engineered (i.e., CNTs) and natural (i.e., BC) nanomaterials, and therefore, it is not known whether soots or CNTs are being released into the ambient environment or workplace. The TGA method described in this thesis should be able to distinguish between BC and SWCNTs, as BC has a much higher thermal stability. Thus, air concentrates on quartz or cellulose filters could be assayed to determine the distribution between CNTs and BC in the collected particles, which should be more quantitative, faster, and cheaper than the current approach (investigation of deposited particles by electron microscopy).

Multi-walled CNTs vs. single-walled CNTs. This investigation has shown that there is a great deal of diversity among materials that are identified as "CNTs." Perhaps the simplest distinction that can be made to classify members of the CNT family is the existence of single or concentric CNT cylinders (SWCNTs and MWCNTs, respectively). While both materials exhibit great industrial promise, the market demand for SWCNTs is expected to expand more than that for MWCNTs, which are currently produced in larger volumes $^{7,8}$ (Table 1). 


\begin{tabular}{lccc} 
\$ Millions & 2004 & 2009 & 2014 \\
\hline TOTAL DEMAND & $\$ 6$ & $\$ 215$ & $\$ 1,070$ \\
\hline
\end{tabular}

\begin{tabular}{lccc} 
BY TYPE & & & \\
\hline Single-walled CNTs & 0 & 95 & 600 \\
Multi-walled CNTs & 6 & 120 & 470 \\
\hline BY END USE & & & \\
\hline Electronics & 0 & 90 & 395 \\
Automotive & 1 & 31 & 165 \\
Aerospace/ Defense & 0 & 10 & 65 \\
Other & 5 & 84 & 445 \\
\hline BY REGION & & & \\
\hline U.S. & 2 & 57 & 290 \\
Western Europe & 1 & 32 & 180 \\
Asia/Pacific & 3 & 113 & 500 \\
Other & 0 & 13 & 100 \\
\hline
\end{tabular}

Table 1. Anticipated growth in the CNT markets by type, end use, and region. Adapted from Thayer $(2007)^{8}$.

Given the future promise of SWCNTs, I initially focused on developing an analytical method for the detection of SWCNTs in the environment (Chapters 2 and 3). As the study progressed, it became apparent that the "packing" of the carbon atoms had an influence on a material's thermal stability. For example, I hypothesized that the higher oxidation temperatures of black carbon (BC) soot (compared to SWCNTs) resulted from reduced oxygen access to the interior shells of carbon present in BC soot. Applying this rationale, I expected the MWCNTs would have a higher thermal stability than SWCNTs. Indeed, the MWCNT sample had a higher thermal stability (Tmax oxidation = $\left.600^{\circ} \mathrm{C}\right)$ than other SWCNTs $\left(\mathrm{T}_{\max \text { oxidation }}=375-540^{\circ} \mathrm{C}\right)$. The higher thermal stability MWCNTs are expected to have decreased interference with sedimentary organic matter (e.g., Dorchester Bay sediment) but an increased interference with black carbon (BC) soot under temperature-programmed oxidation (TPO). Thus, while it may be possible to 
distinguish between SWCNTs and MWCNTs in mixed samples using thermal techniques, it might be difficult to isolate MWCNTs from BC soot. As MWCNTs have been produced for over twenty-five years ${ }^{c}$, and since they dominate the current CNT market, it is critically important that MWCNT analytical methods are developed alongside techniques for the detection of SWCNTs in the environment.

Currently, the majority of CNTs manufactured worldwide are MWCNTs ${ }^{7}$, and the laboratory-scale CNT reactor used in this study was designed to produce MWCNTs (Chapters 4 and 5). The principle difference between MWCNT and SWCNT production by CVD is the choice of catalyst, and hydrocarbon precursors (or CO) are heated to high temperatures in both cases. Since this study focused on thermal rearrangements of the feedstock gas, rather than catalyst-mediated transformations, I expect that a similar product distribution would be observed in SWCNT syntheses that involve heating hydrocarbons to high temperatures. Furthermore, I expect that alkyne-assisted CVD growth will reduce the formation of dangerous VOC and PAH emissions regardless of whether MWCNTs or SWCNTs are desired, since the thermal pre-treatment of the hydrocarbon precursor is not necessary with this approach.

The proposed CNT formation mechanism presented in Chapter 5 is generic and does not consider differences between SWCNTs and MWCNTs. The choice to illustrate only one growing CNT wall is for clarity's sake, and it is not meant to imply that there are no differences between the two formation processes. Indeed, I expect that there critical distinctions between the synthesis of SWCNTs and MWCNTs. For example, the transport of carbon to the catalyst may be different in SWCNT production compared to MWCNT production. Carbon supply to interior walls of a growing MWCNT could proceed via (1) diffusion through the catalyst, (2) migration from outer CNT walls to inner CNT walls, or (3) through a precursor shuttling mechanism between catalytic metal centers from the outside of the MWCNT to its inner walls. Clearly, there are important implications for the CNT formation mechanisms, and these will have to be investigated

\footnotetext{
${ }^{\mathrm{c}}$ Hyperion Catalysis in Cambridge, MA has been producing MWCNTs for sale in CNTdoped polymers since 1982, referring to the materials as carbon "FIBRILSTM."
} 
with careful consideration of physical transport processes, as well as chemical reactivity at the metal catalyst. Nevertheless, I expect that the enhanced chemical reactivity of alkynes toward CNT formation that was observed for MWCNTs in Chapter 5 will hold for SWCNT production. Indeed, much more study is needed before the mechanisms controlling the formation of SWCNTs and MWCNTs are truly understood.

\section{CRITICISM}

Historically, industrial engineers and environmental chemists have had competing interests, where the former feel unjustly scrutinized and policed by the latter. This "good vs. evil" paradigm is deep-rooted and substantial inertia prevents both parties from overcoming these traditional barriers. Here, I endeavored to work with industry, mechanical engineers, and materials scientists to design materials and production practices in a safe way. While somewhat cooperative, most of my collaborators were skeptical of my results. The primary criticisms were that (1) not all CNT manufacture by CVD uses the same feedstock gas, (2) some manufacturers recycle their effluents, (3) a laboratory-scale synthesis is not representative of an industrial-scale production, and (4) other typical processes (e.g., driving cars) contribute more VOC and PAH emissions than will CNT production. All of these points are valid and justified, but they are not sufficient grounds to avoid investigating and mitigating the environmental ramifications of production practices early in the design phase of novel materials. I will respond to these here:

(1) While not all CNT production relies on the same feedstock gas, many employ a hydrocarbon precursor. I would argue that high-temperature gas phase reactions of these compounds have relatively generic mechanisms, and while the quantities of compounds might vary (with hydrogen content and temperature, for example), many of the same compounds will be formed. (Note that a synthesis that does not employ a hydrocarbon, but instead, $\mathrm{CO}$, may have very different reaction products). Further, I strongly suggest that each manufacturer use adequately sensitive (e.g., not online mass 
spectrometry by residual gas analysis) techniques to assess the content of their effluent. Their data would provide stronger evidence to either confirm or deny the supposition that they are not polluting. Thus, I am not suggesting that my results apply to all cases, but rather that each production be evaluated on a case-by-case basis. (This will have the added benefit of uncovering the most environmental benign of the synthetic approaches).

(2) Although it is true that some manufacturers recycle their effluent (at least two of ten US manufacturers do and many European companies adopt the practice), at the end of a days production, effluents are vented. Thus, releases may be non-trivial. Also, in some effluent recycling schemes, the feedstock gas of interest (e.g., $\left.\mathrm{C}_{2} \mathrm{H}_{4}\right)$ is captured while other materials are "flamed." Flaming is intended to convert potentially dangerous materials to $\mathrm{CO}_{2}$ (which we know is not environmentally benign). However, just as there are few studies related to the content of the effluent, there are even fewer studies that assay the products of the flame and optimal flame conditions may not be established. The result is that incomplete combustion processes could lead to the generation of "natural" nanoparticles (e.g., BC soot) or PAHs, similar to those that result from forest fires and combustion engines. Simple inspection of a flame stack from a CNT production facility illustrates this point (Figure 2, note visible black particles at crest of flame).

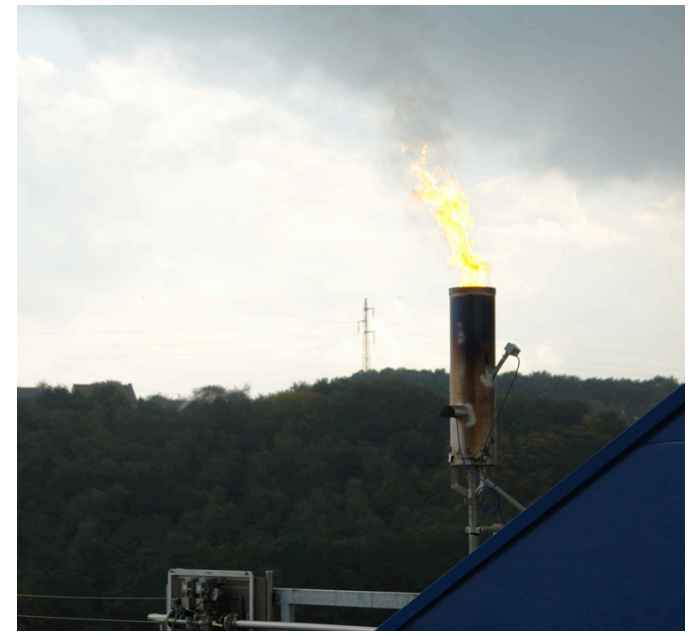

Figure 2. The flame torch used at a CNT production facility intended to convert pollutants or toxicants to $\mathrm{CO}_{2}$. It is clear from the picture that particle formation is taking place, and it is likely that PAHs will be formed here also. At this factory, $\mathrm{C}_{2} \mathrm{H}_{4}$ is the feedstock gas for the CVD synthesis of CNTs, but this is trapped and reused, not flamed. 
(3) Perhaps the most common criticism of this work is that a laboratory-scale synthesis is not representative of an industrial scale production. I agree that that is true, and I will sample at a production plant someday. However, I would argue (as I did in point 1) that the formation mechanisms may be similar between analogous syntheses (e.g., temperature and feedstock gas choice similar) and though the quantities might change, the distribution of chemicals may be comparable. Second, production practices are continually evolving, and processes measured today will likely be different a year from now. Thus, any projection of future emissions due to industrial scale CNT synthesis will be flawed (even when one doesn't consider the large uncertainties in anticipated production volumes). Nevertheless, these projections can help guide the evolution of the design process. That is, one could estimate potential emissions from several different synthetic scenarios and select the practice that has minimal potential to have a negative impact on the environment or public health and maximum production benefits (e.g., minimum cost, maximum product performance). In this way, environmentally dangerous practices can be avoided before significant financial investments result in a static infrastructure that is steadfastly routed in unsound, unsustainable production practice. Finally, to argue that early evaluations of laboratory scale processes are an invalid way to gauge potential environmental impact is essentially arguing that early environmental assessment should not be conducted at all. (There is no alternative to determine potential impacts if early measurements are not taken). It is not possible to predict the future synthetic approach, volume, or location of production, and so, if one waits until the industry is fully expanded, she will be too late to effect PREVENTATIVE measures. While $100 \%$ accurate extrapolations to the future are not likely, it is possible to forecast to the best of our ability and endeavor to avoid irreparable damage to human and environmental health.

(4) The argument that pollution is condonable when there are others nearby who pollute in larger quantities can be flawed. While large investments to avoid small (e.g., $<<1 \%$ ) inputs to the total industrial pollution burden in a city are often unjustifiable, more substantial contributions could result in deteriorated public or environmental health. 
For example, Houston, TX has the United States' largest petrochemical refineries and also some of the highest benzene and 1,3-butadiene levels in the country (1,3-butadiene concentrations are 20 times higher than in any other US city ${ }^{9}$ ). Recent studies (i.e., 19952004 data, 2008 publication $^{10}$ ) have shown that children (but not adults) living within the Houston shipping channel had a $60 \%$ higher chance of contracting leukemia than children living in clean-air areas, and this cancer incidence correlated to benzene and 1,3butadiene concentrations in the air. In response to that study, the Mayor of Houston hired environmental consultants and lawyers to demand immediate reductions in industrially derived VOCs. My estimates suggest that moving a reasonable fraction of CNT production ( $20 \%$ of the production from only two of the myriad potential applications) to Houston would more than double 1,3-butadiene emissions and increase benzene emissions by $20 \%$. Clearly, such increases would be unacceptable to Houston's environmental task force and should be unacceptable to anyone who believes that children should not be made sick as a result of where they live.

It is important to note that over 90\% of the population living in Houston's shipping channel is Hispanic ${ }^{11}$. Thus, failure to protect all members of society from industrial contaminants could quickly become an issue of social injustice, as well as environmental negligence. 


\section{PRECEDENT}

As a nascent technology, the CNT industry is continually evolving. Thus, the CNTs measured in this study may not be the CNTs that are used 10 years from now, and the production methods will likely undergo change as well. The analytical method I outlined here will require additional "tuning" to optimize for detection in environmental samples, and the emissions estimates will almost certainly diverge from those that result from increased global CNT production. However, the procedures and approaches described in this work are valid and should be continually repeated as the industry expands, in order to inform best use practices as they develop. In particular, I emphasize that we must (1) adopt a proactive approach to prevent the indiscriminant use and inadvertent release of potentially harmful chemicals and (2) work with industry and materials engineers to co-optimize synthetic parameters for both product performance and environmental objectives. This would represent a departure from traditional interactions between environmental chemists and industrial engineers, who have had antagonistic relationships focused on chemicals that have already done significant damage to the environment and public health. Thus, it is my hope that this work will not only ensure the safe and conscientious advancement of the CNT industry, but also serve as a model for the early, sustainable, collaborative development of all novel materials. 


\section{OPPORTUNITIES}

There is a great deal of uncertainty surrounding the role that carbon nanotubes will play in the environment and much work remains before we are able to predict, $a$ priori, the influence, partitioning, and lifetime of CNTs in the environment. Given the breadth of the thesis, I will organize the most pressing ensuing studies in reference to thesis chapters.

\section{Chapter 2. Characterization.}

CNT reactivity. PAHs are formed during carbon nanotube synthesis from ethene (Chapter 4), and CNTs, which are extremely hydrophobic, should adsorb some of these PAHs from the gas phase. Indeed, PAHs are coated with an "amorphous carbon" phase. However, dichloromethane (DCM) extractions of CNTs yield sparse quantities of PAHs. Low recoveries of aromatic internal standards in CNT suspensions (but not CNT free controls) suggest that CNTs (or some DCM-CNT interaction) are acting to degrade the aromatic compounds. If so, then CNTs may have some ability to degrade pollutants in the environment. On the contrary, if the mechanism by which the CNTs degraded the internal standards involved a DCM-derived free radical, then CNTs in contact with chlorinated contaminants in the environment could produce even more toxic materials. Since there is interest in using CNTs to purify surface drinking and ground water, it will be very important to understand these possible reactions prior to introducing the materials to the human drinking water supply.

Isotopic fractionation. The distribution of stable carbon isotopic signatures in commercially available CNTs appeared to be related to the expected ${ }^{13} \mathrm{C}$ content of their starting materials (i.e., carbon feedstock gas or graphite rod). As most CNT formation reactions take place at very high temperature (e.g., $500-1200^{\circ} \mathrm{C}$ ), it is reasonable to expect that there is very little isotopic fractionation from the starting material to the CNT product. However, given the stability of the aromatic bonds in CNTs, it is possible that there is an isotopic fractionation. If so, this might provide valuable insight into the CNT formation mechanism, and such fractionation processes should be explored. 
Chapter 3. Analytical method for CNTs in the environment. There are several properties of CNTs that might distinguish them from other natural organic matter and allow their quantification in the environment. For example, CNTs have remarkable mechanical strength and heat transfer properties. Single CNTs are said to improve the performance of thermal interface materials ${ }^{12}$, and if that is true, then it is possible that thermal conductance measurements could detect single CNTs. Similarly, very low masses of CNTs are reported to afford increased mechanical strength in composite materials. Mechanical tests on native and CNT-doped sediments might indicate the presence of CNTs in sedimentary samples (although it is likely that such an analysis would require a CNT-free sediment end member to quantify the CNTs). Fluorescently labeled anti-bodies against CNT agglomerates might enable visualization of CNTs and eventual quantification using the fluorescence intensity. Antibodies are roughly $10 \mathrm{~nm}$ in size, and CNT agglomerates (but not single SWCNTs) should be big enough to elicit an immune response.

In addition to providing an analytical tool to detect CNTs, TGA could be used as a rapid, minimum interference purification step for isolating CNTs from CNT-organic matter mixtures. For example, investigations of CNT behavior in the natural aquatic systems will inevitably require CNTs to be in close association with natural organic mater $(\mathrm{NOM})^{13}$. NOM has a demonstrated ability to generate high concentrations of singlet oxygen in adjacent microenvironments ${ }^{14, \mathrm{~d}}$, and CNT surfaces may be oxidized by NOM-derived singlet oxygen. This functionalization of the CNT surface has clear and significant implications for the environmental transport of the material, as the addition of oxygen-containing moities will increase the hydrophilicity of an otherwise hydrophobic nanoparticle. In order to determine whether or not CNTs are functionalized by NOMgenerated singlet oxygen, the CNTs will have to be separated from NOM following any

d There is speculation that CNTs can generate ${ }^{1} \mathrm{O}_{2}$ independently. The excited state energies of select CNTs are inversely related to their diameter (e.g., $1.36 \mathrm{eV}$ for $0.7 \mathrm{~nm}$ and $0.80 \mathrm{eV}$ for $1.3 \mathrm{~nm} \mathrm{CNTs})^{15}$. Thus, energy transfer to triplet oxygen to form ${ }^{1} \mathrm{O}_{2}(1.07$ eV singlet-triplet gap) is out of reach for all but the smallest CNTs, with diameters below approximately $0.9 \mathrm{~nm}$. 
experimentation. Presuming that NOM has a lower thermal stability than CNTs, one could oxidize the NOM at a low temperature at which CNTs are preserved. The CNTs could then be recovered and interrogated by x-ray photoelectron spectroscopy for surface functionality and aggregation kinetics of the resultant, modified CNTs could be assayed using a dynamic light scattering technique. Kinetic parameters from the oxidative aging and aggregation experiments will be very useful to improve CNT mass balance models, such as that presented in Chapter 1 (which assumes a static, hydrophobic nanoparticle).

Chapter 4. Effluents formed during CNT synthesis. The solid carbon forms generated during CVD include (1) the CNTs themselves, which are typically fixed to the growth substrate, (2) soot that may form and exit the reactor, and (3) solid carbon deposits on the walls of the pre-heater tube. The latter solid carbon forms were not identified, but I am confident that they are not typical BC soot. I oxidized the material in air at $450^{\circ} \mathrm{C}$ for over $24 \mathrm{hrs}$, and it did not degrade. The material did oxidize in a closed tube combustion at $850^{\circ} \mathrm{C}$ for 5 hours over cupric oxide (as an oxygen source). This suggests that the material is not BC soot, but may be some more stable material. Its color was not as dark as BC soot, but instead, a shimmering gray color (similar to graphite or graphene). In syntheses where the pre-heater is on, this material represents the largest sink for the feedstock ethene, and thus, it may be important to identify. (Note, the material is not formed during unheated (e.g., alkyne-asisted) syntheses).

Filters collected at the effluent should be analyzed for soot and CNT content. In particular, a CNT synthesis where free-floating (rather than substrate-affixed) catalyst are employed, should be investigated to determine if CNT release is more likely when CNTs are not affixed to a substrate-bound growth catalyst.

Chapter 5. Improved resource use for CNT manufacture. The principle limitation of the reactor used in this study is that there is only physical space for one catalyst piece (at most $1 \mathrm{~cm}^{2}$ ). While I expect (as it was relatively facile to limit CNT growth with a $20 \%$ reduction in $\mathrm{C}_{2} \mathrm{H}_{4}$ input) that we are operating near the optimal Cprecursor: metal catalyst ratio, it is possible that more nanotubes could be grown from the 
same amount of carbon feedstock gas. Improvements in the $\mathrm{C}$ feedstock: metal catalyst would offer immediate improvements in the reaction efficiency.

As mentioned earlier, I hypothesize that the amorphous carbon coating formed on CNTs is partly due to the presence of PAHs in the CNT reactors. Using unheated, alkyne-assisted reactions, I expect that the carbon purity of CNTs (e.g., CNT to amorphous $\mathrm{C}$ ratio) will increase. This should offer an improvement in the electrical conductivity of the sample, and thus, product performance and environmental optimization would have been achieved simultaneously.

While we are still far from understanding the exact reaction mechanism of CNT formation, the recent results highlight areas for future exploration. Our proposed mechanism offers a potential route to chirality observed in real world CNTs. This chirality could be controlled by the initial ring formation between an alkene and an alkyne and subsequent additions that align with the initial chirality established. I am interested to see if different sterically hindered precursors with terminal alkynes will yield CNTs of different chiral wrapping.

More specifically, in the ring formation mechanism between an alkene and a terminal alkyne, carbon chains that dangle from the terminal alkyne (e.g., the alkene group at the end of but-1-en-3-yne) generally stick out of the plane of the ring. If this were true, one would not expect a significant difference in the reactivity of 1-butyne and but-1-en-3-yne. However, a difference in the reactivity of these compounds was observed, and that suggests that the double bond on the head of but-1-en-3-yne is involved in the chain elongation reaction. If so, I would expect the whole but-1-en-3-yne molecule to deposit in the lattice of the growing nanotube (i.e., all 4 carbon atoms are in the aromatic system of the CNT). The distinction between these two reactions mechanisms could be tested with either (1) deuterated test compounds (e.g., $d_{4}$-but-1-en3-yne) and $\delta^{2} \mathrm{H}$ isotopic values of $\mathrm{H}_{2}$ evolved during the $\mathrm{CNT}$ formation reaction and/or (2) infrared spectroscopy to determine function groups protruding from the CNT backbone. If the whole but-1-en-3-yne molecule folds into the CNT lattice, then all of the hydrogen atoms (or deuterium atoms) must be removed from the original carbon 
chain and these would show up in the gas phase of the $\mathrm{H}_{2}$ evolved during the reaction. In contrast the deuterium atoms will remain bound to the tail of the molecule and dangle from the CNT structure. Whether deuterium or hydrogen, the C-H (or C-D) stretches due to these atoms should be visible using spectroscopic techniques.

Irrespective of a mechanistic understanding, I have shown that measurable improvements in CNT synthetic approach can be made early on in the design process. In addition, there is promise toward the development of a sensitive analytical technique for CNTs in the environment. These results will improve our ability to study and then predict the long-range transport and human and ecological exposure to CNTs. This will become increasingly important as the toxicity of these materials is established. With an understanding of nanoparticle fate, informed choices for CNT use in consumer products can be made. In this way, we may prevent unacceptable ecological and human health consequences, and thereby alter the, so far, unfortunate trajectory of industrial chemicals in the Earth system. 


\section{REFERENCES}

[1] Lam, C-W.; James, J.T.; McCluskey, R.; Hunter, R.L. Pulmonary toxicity of single-wall carbon nanotube in mice $7 \& 90$ days after intratracheal instillation. Toxicol. Sciences. 2004, 77, 126-134.

[2] Jia, G.; Wang, H.; Yan, L.; Wang, X.; Pei, R.; Yan, T.; Zhao, Y.; Guo, X. Cytotoxicity of carbon nanomaterials: single-wall nanotube, multi-wall nanotube, and fullerene. Environ. Sci. Technol. 2005, 39, 1378-1383.

[3] Poland, C.A.; Duffin, R.; Kinloch, I.; Maynard, A.; Wallace, W.A.H.; Seaton, A.; Stone, V.; Brown, S.; MacNee, W.; Donaldson, K. Carbon nanotubes introduced intot he abdominal cavity of mice show asbestoslike pathogenicity in a pilot study. Nature Nanotechnology 2008, 3, 423-428.

[4] Templeton, R.C.; Ferguson, P.L.; Washburn, K.M.; Scrivens, W.A.; and Chandler, G.T. Life-cycle effects of single-walled carbon nanotubes (CNTs) on an estuarine meiobenthic copepod. Environ. Sci. Technol. 2006, 40 (23), 7387-7393.

[5] Guo, L.; Morris, D.G.; Liu, X.; Vaslet, C.; Hurt, R.H.; Kane, A.B. Iron bioavailability and redox ativity in diverse carbon nanotube samples. Chem. Mater. 2007, 19 (14), 3472-3478.

[6] Kang, S.; Mauter, M.S.; Elimelech, M. Physicochemical determinants of multiwalled carbon nanotube bacterial cytotoxicity. Environ. Sci. Technol. 2008, 42, 7528-7534.

[7] Eklund, P.C.; Ajayan, P.M.; Blackmon, R.; Hart, A.J.; Kong, J.; Pradhan, B.; Rao, A.; Rinzler, A.G. International Assessment of Research and Development on Carbon Nanotubes: Manufacturing and Applications, World Technology Evaluation Center Report, 2008, http://www.wtec.org/cnm.

[8] Thayer, A.M. Carbon nanotubes by the metric ton. Chemical \& Engineering News 2007, 85 (46), 29-35.

[9] Fraser, M.P.; Birnur, B. Source identification and apportionment of volatile organic compounds in Houston, TX. Atmos. Environ. 2006, 40, 23852400 .

[10] Whitworth, K.W.; Symanski, E.; Coker, A.L. Childhood lymphohematopoietic cancer incidence and hazardous air pollutants in 
Southeast Texas, 1995-2004. Environmental Health Perspectives 2008, 116 (11), 1576-1580.

[11] McGarity, T. Professor of Environmental Law at the University of Texas, quoted in Fox, M.A.; "Mother fears 'stinky neighborhood' cause sons cancer," http://www.studentnews.cnn.com/2007/US/10/18/poor.environment/index. html. Accessed Feb 2009.

[12] Personal communication, Intel ${ }^{\mathrm{TM}}$, November 2008.

[13] Hyung, H.; Fortner, J.D.; Hughes, J.B.; Kim, J-H. Natural organic matter stabilizes carbon nanotubes in the aqueous phase. Environ. Sci. Technol. 2007, 41, 179-184.

[14] Latch, D.E.; McNeill, K. Microheterogeneity of singlet oxygen distributions in irradiated humic acid solutions. Science 2006, 311 (5768), 1743-1747.

[15] Buchilo, S.M.; Strano, M.S.; Kittrell, C.; Hauge, R.H.; Smalley, R.E.; Weisman, R.B. Structure-assigned optical spectra of single-walled carbon nanotubes. Science 2002, 298 (5602), 2361-2366. 
Build today, then strong and sure, with a firm and ample base; and ascending and secure, shall tomorrow find its place.

-Henry Wadsworth Longfellow, a son of Portland, Maine 